

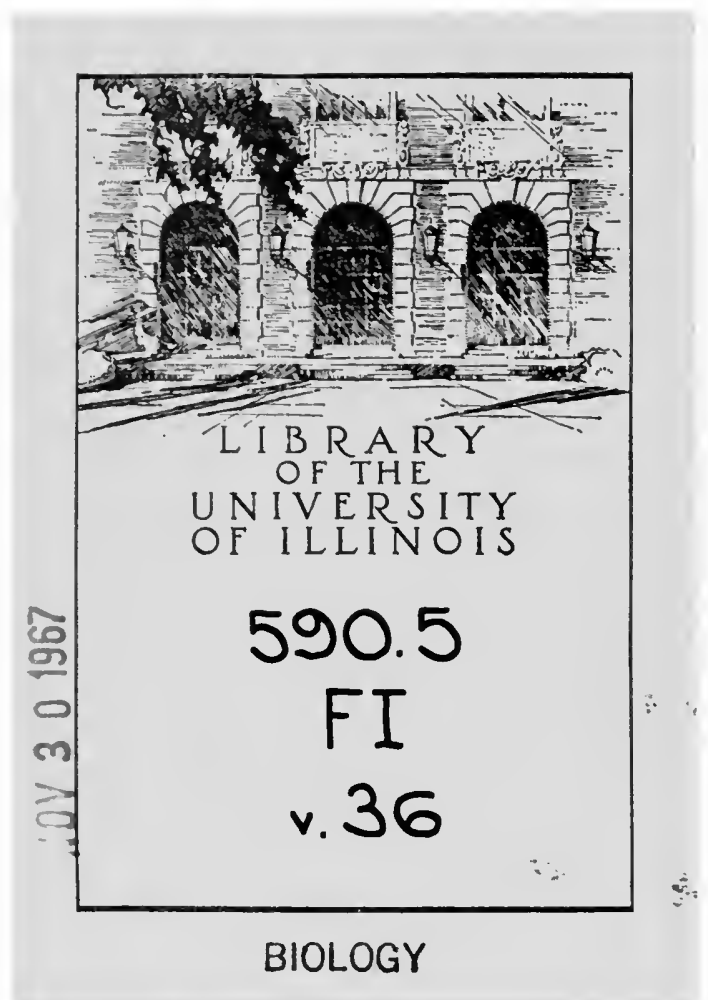


The person charging this material is responsible for its return on or before the Latest Date stamped below.

Theff, mutilation, and underlining of books are reasons for disciplinary action and may result in dismissal from the University.

University of Illinois Library

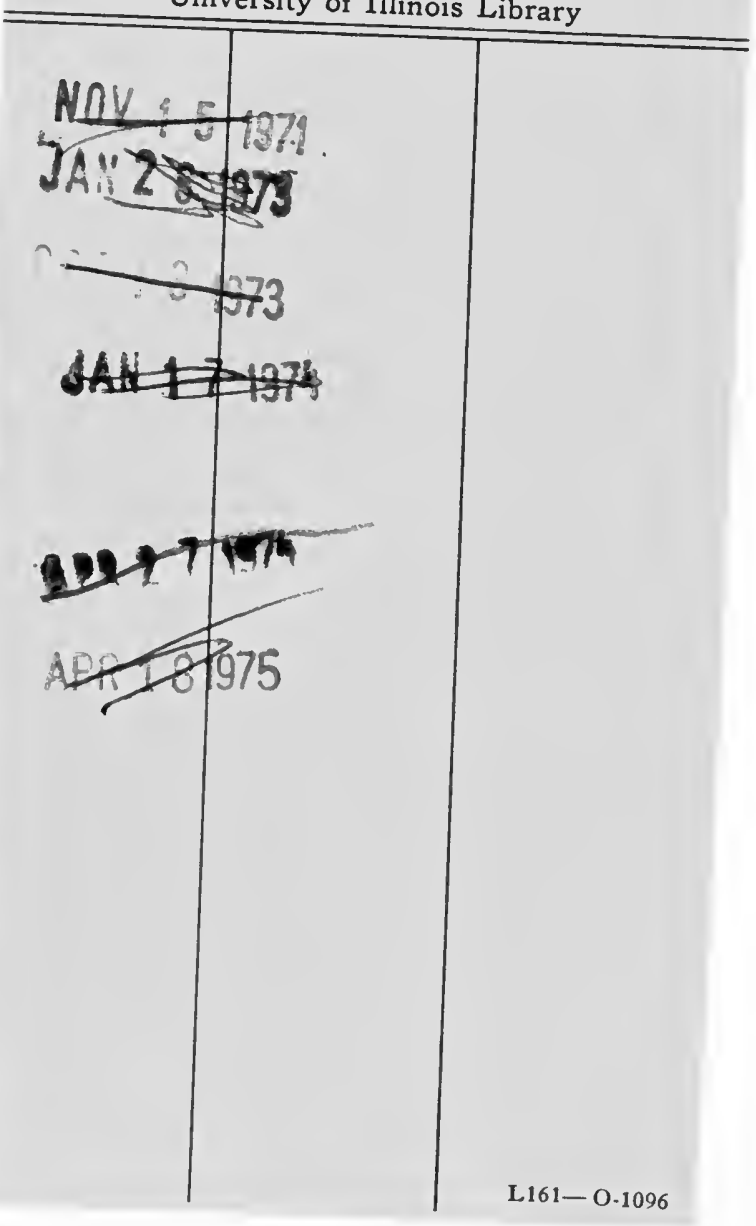




r 


\title{
THE DISTRIBUTION OF FISHES FOUND
}

\section{BELOW A DEPTH OF 2000 METERS}

\author{
MARION GREY
}

FIELDIANA : ZOOLOGY

VOLUME 36, NUMBER 2

Published by

CHICAGO NATURAL HISTORY MUSEUM

JULY 30,1956 





\title{
THE DISTRIBUTION OF FISHES FOUND
}

\section{BELOW A DEPTH OF 2000 METERS}

\author{
MARION GREY \\ Associate, Division of Fishes
}

FIELDIANA : ZOOLOGY VOLUME 36 , NUMBER 2

TWE LIBRARY OF THE

AUG 141456 UNNERETT OF ILUNOLS

Published by

CHICAGO NATURAL HISTORY MUSEUM

JULY 30,1956 
PRINTED IN THE UNITED STATES OF AMERICA

BY CHICAGO NATURAL HISTORY MUSEUM PRESS 


\section{CONTENTS}

INTRODUCTION . . . . . . . . . . . . . 77

Terminology . . . . . . . . . . . . . . 78

Fishes found below 3660 meters . . . . . . . . . . 78

Distinctive character of deep-abyssal fauna . . . . . . . 82

Endemism in deep-abyssal waters . . . . . . . . . . 83

Endemism of species . . . . . . . . . . . 84

The bathypelagic fishes ............ . . 88

Conclusion . . . . . . . . . . . . . . . 92

Note . . . . . . . . . . . . . . . . 93

Editorial note . . . . . . . . . . . . . . 93

Acknowledgments ................... 93

SYNONYMIES AND DISTRIBUTION .................... 94

Scylliorhinidae ..................... 94

Squalidae . . . . . . . . . . . . . 95

Rajidae . . . . . . . . . . . . . . 98

Chimaeridae ................ . . . 100

Rhinochimaeridae ............... . . 101

Alepocephalidae . . . . . . . . . . . . 102

Searsiidae . . . . . . . . . . . . 116

Gonostomatidae . . . . . . . . . . . . . 119

Bathylaconidae . . . . . . . . . . . . . . 127

Harpadontidae . . . . . . . . . . . . . . 128

Chlorophthalmidae . . . . . . . . . . . . . 129

Bathypteroidae . . . . . . . . . . . . 130

Ipnopidae . . . . . . . . . . . . . . . . . 135

Eurypharyngidae . . . . . . . . . . . . 137

Simenchelyidae . . . . . . . . . . . . . . . . . 139

Nettastomidae . . . . . . . . . . . . . 140

Congridae . . . . . . . . . . . . . . 142

Ilyophidae . . . . . . . . . . . . . . . . . 142

Synaphobranchidae . . . . . . . . . . . . . 143

Serrivomeridae . . . . . . . . . . . . . 148

Nemichthyidae . . . . . . . . . . . . . . . . . 149

Cyemidae . . . . . . . . . . . . . . 151 
Halosauridae . . . . . . . . . . . . . . . 152

Notacanthidae . . . . . . . . . . . . . . 156

Moridae . . . . . . . . . . . . . . . . 158

Gadidae ................... . . . 161

Macrouridae . . . . . . . . . . . . . . . 162

Stephanoberycidae . . . . . . . . . . . . . 190

Melamphaidae .. . . . . . . . . . . . . . 191

Acropomatidae(?) . . . . . . . . . . . . . . . . 192

Parapercidae . . . . . . . . . . . . . . . . . 193

Chiasmodontidae . . . . . . . . . . . . . 193

Bathydraconidae .. . . . . . . . . . . . . . . . . . 194

Zoarcidae ...................... 194

Lycodapodidae . . . . . . . . . . . . . . . 201

Derepodichthyidae ................. 202

Brotulidae . . . . . . . . . . . . . . . . . . 202

Scorpaenidae . . . . . . . . . . . . . . . 222

Cottidae . . . . . . . . . . . . . . . . 222

Cottunculidae . . . . . . . . . . . . . . . 223

Liparidae . . . . . . . . . . . . . . . . . 223

Triacanthodidae . . . . . . . . . . . . . . . . 228

Chaunacidae . . . . . . . . . . . . . . . 229

Ogocephalidae . . . . . . . . . . . . . . . . 229

Ceratioidea ................... . . 2 . . . . . . . . . . . . .

Caulophrynidae . . . . . . . . . . . . . . . . . . . 232

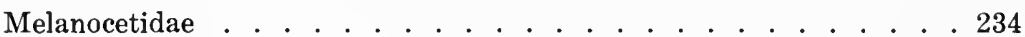

Himantolophidae . . . . . . . . . . . . . . . . . . 239

Diceratiidae . . . . . . . . . . . . . . . 242

Oneirodidae . . . . . . . . . . . . . . . . 243

Centrophrynidae . . . . . . . . . . . . . . . 261

Ceratiidae . . . . . . . . . . . . . . . . 261

Gigantactinidae . . . . . . . . . . . . . . . 266

Neoceratiidae . . . . . . . . . . . . . . . . 269

Linophrynidae . . . . . . . . . . . . . . . . . . 270

Tables Showing Vertical Distribution of Species . . . . . . . . 280

List of Benthic Fishes Found Below 1000 Fathoms . . . . . . . . . 304

List of Bathypelagic Fishes Found Below 1000 Fathoms . . . . . . . 311

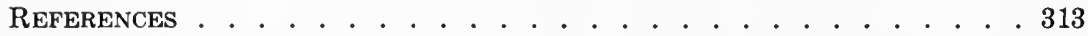

ADDENDUM . . . . . . . . . . . . . . . . . . . . 320

INDEX . . . . . . . . . . . . . . . . . . . 321 


\section{The Distribution of Fishes Found Below a Depth of 2000 Meters}

\section{INTRODUCTION}

In The Depths of the Ocean (Murray and Hjort, 1912, p. 414) Dr. Johan Hjort published a list of fishes believed to live upon the ocean floor at depths exceeding 2000 fathoms (3660 meters). The list contained twenty-one species belonging to six families, with only thirty-five individuals known.

A check of Hjort's list in the light of subsequent knowledge reveals that two of the species, Bathypterois longicauda Günther and Macrurus gigas Vaillant, have been reduced to synonymy, and that one, Histiobranchus infernalis Gill, was erroneously included and has never actually been reported from waters below 3660 meters. Aleposomus copei Gill is undoubtedly a bathypelagic fish, with its center of distribution well above the 2000 -meter line. Thus, of the species listed by Hjort, only seventeen that can with any degree of certainty be referred to as benthic fishes are found below a depth of 3660 meters.

A survey of the literature published since 1912 adds to Hjort's list twenty-one species that probably live on bottom, thus raising the present total to a possible thirty-eight species, divided among eleven families and thirty genera, and represented by about 120 specimens actually caught below 3660 meters. The increase is small, but the technical difficulties and costliness of fishing at such great depths have not lessened in the intervening years and little such work has been carried out, especially in comparison with the large amount of fishing accomplished at higher levels in the deep sea. There are still some unidentified collections made by the United States Fisheries Steamer Albatross in Pacific waters, as well as recent collections made by Swedish, Russian, and Danish oceanographic expeditions. Dr. A. F. Bruun has written (1952, in litt.) that the results of the Galathea Expedition (1950-52) will alter some of the findings noted in this paper, extending the ranges of 
some of the species listed here and adding new species to the list. The Danish expedition concentrated on abyssal benthic trawling in many different parts of the world, returning with a large collection of fishes and invertebrates.

Although the Galathea results and other reports will add greatly to our knowledge, much further research will be necessary before we can attain a clear or complete picture of life at these great depths. Research on deep-sea life is still in its infancy. New forms are found so frequently that more obviously remain to be discovered, and it will be many years before enough benthic material has been gathered from deep-abyssal waters to allow detailed distributional studies.

\section{TERMINOLOGY}

The terms archibenthic (between 200-400 and about 1000 meters) and abyssal (below 1000 meters) are used here as defined by Ekman $(1935,1953)$. The name deep-abyssal has been added to denote those areas of the sea lying below a depth of about 2000 meters. Nybelin (1953) has proposed the term eu-abyssal for the fauna found below about 4000 meters.

Hjort defined the abyssal plain as those areas of the ocean floor below a depth of 2000 fathoms (3660 meters), the regions between 1500 and 2000 fathoms being, for fishes, a transition zone between the plain and the continental slope. It seems useful to include among the deepest-living fishes those found below a depth of 2000 meters, and this deep-abyssal fauna is treated in the following discussion. Species ranging to 1000 fathoms (1829 meters) are included, since the 1000-fathom line has been used as often as the 2000-meter line to divide deep-sea zones. These divisions are arbitrary in any case. It is known that the vertical boundaries of deep-sea zones vary considerably in different areas but inadequate knowledge of the fauna prevents a precise definition of these zones.

\section{FISHES FOUND BELOW 3660 METERS}

Hjort pointed out that benthic species recorded from the abyssal plain, and also known from more than one catch, have wide ranges both vertically and horizontally. Thus, Nematonurus armatus has an extreme vertical range of 282 to 4700 meters. Its geographical distribution is also wide, specimens having been reported from both sides of the Atlantic, in the central and southern parts of the Pacific, and in the southern Indian Ocean. Similar examples will be found in the discussion of individual species, but there are also species 
known from several hauls of which none were above the deep-abyssal zone (Bathysaurus mollis, Synaphobranchus bathybius, Lionurus filicauda and others). Bathymicrops regis, Bassozetus taenia and Grimaldichthys profundissimus have been caught several times each, and only on the abyssal plain. These three species have been taken only in the north Atlantic and are not, as yet, known to have a wide

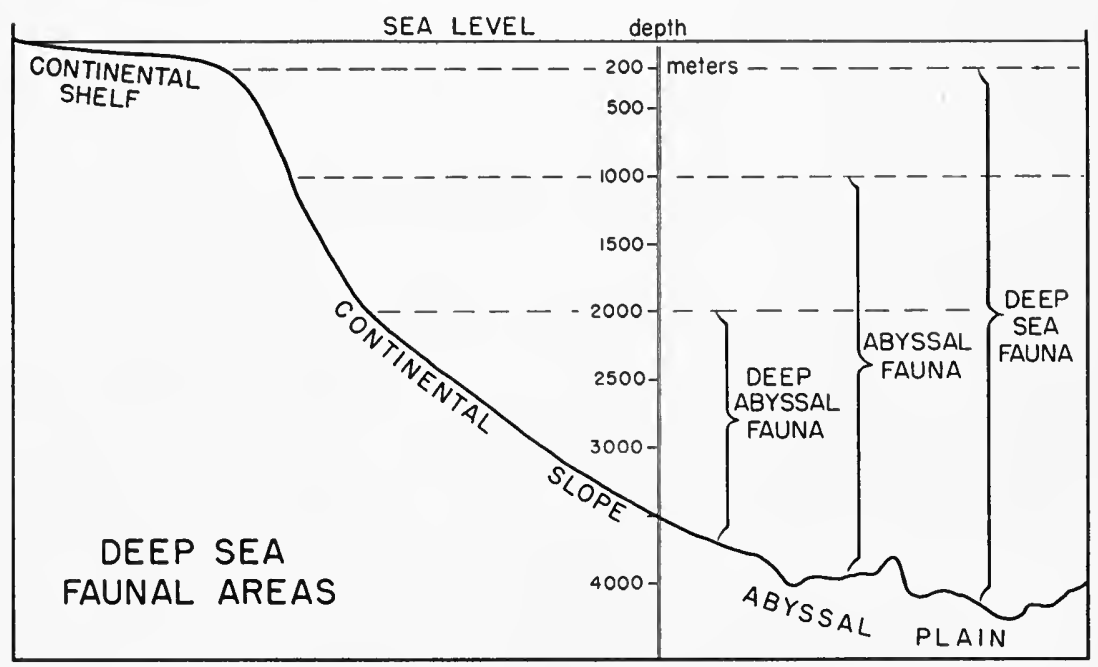

Fig. 4. Deep sea faunal areas.

horizontal range. At the present time any attempt to outline patterns of fish-distribution on the abyssal plain would be open to question.

Of the fishes whose vertical range includes the abyssal plain below 3660 meters, nineteen of the species listed below (names preceded by an asterisk) are known from only one, two, or three specimens each. They may actually be relatively common at such depths but further captures will be necessary before that fact can be established. Of the remaining species on the list, only Bathysaurus mollis, Bathymicrops regis, Nematonurus armatus, and perhaps Bassozetus taenia and Grimaldichthys profundissimus, show evidence of being more than accidental on the abyssal plain.

\section{Family ALEPOCEPHALIDAE}

Alepocephalus rostratus Risso ${ }^{1}$

${ }^{*}$ Rinoctes nasutus Koefoed

${ }^{*}$ Bathytroctes macrolepis Günther
Rouleina attritus Vaillant ${ }^{1}$

*Ericara salmonea Gill and Townsend

${ }^{1}$ Deepest record 3655 meters. Listed by Murray and Hjort (1912) but probably not reaching the abyssal plain. 


\section{Family BATHYLACONIDAE}

${ }^{*}$ Bathylaco nigricans Goode and Bean

Family HARPADONTIDAE

Bathysaurus mollis Günther

Family BATHYPTEROIDAE

Bathypterois longipes Günther

\section{Family IPNOPIDAE}

Bathymicrops regis Hjort and Koefoed Ipnops murrayi Günther

*Bathymicrops sewelli Norman

\section{Family SYNAPHOBRANCHIDAE}

*Synaphobranchus bathybius Günther

Family HALOSAURIDAE

*Aldrovandia rostrata Günther

\section{Family MACROURIDAE}

*Nematonurus abyssorum Gilbert

Nematonurus armatus Hector

*Chalinura liocephala Günther

Chalinura simula Goode and Bean

Chalinura carapina Goode and Bean
Chalinura brevibarbis Goode and Bean Lionurus filicauda Günther

Nezumia sclerorhynchus Valenciennes ${ }^{1}$

Cetonurus globiceps Vaillant

*Echinomacrurus mollis Roule

\section{Family STEPHANOBER YCIDAE}

Acanthochaenus luetkeni Gill

*Malacosarcus macrostoma Günther

\section{Family ZOARGIDAE}

*Lycenchelys albus Vaillant

*Pachycara obesa Zugmayer

\section{Family BROTULIDAE}

*Barathrites abyssorum Roule

Bassogigas crassus Vaillant

Bassogigas digittatus Garman

*Bassogigas brucei Dollo

*Alcockia rostrata Günther

Bassozetus taenia Günther
Mixonus laticeps Günther Grimaldichthys profundissimus Roule

${ }^{*}$ Grimaldichthys squamosus Roule

*Typhlonus nasus Günther

*Leucochlamys cryptophthalmus Zugmayer

The following species have not been included in the above list because in each case the one record from below 3660 meters must be ignored in the face of overwhelming evidence that the species are well known at much higher levels: Hymenocephalus italicus Giglioli,

${ }^{1}$ See footnote, page 79 . 
discussed below with the fauna found below 2000 meters; Dicrolene introniger Goode and Bean, a species of shallower water, of which one damaged specimen has been reported from 5000 meters (Koefoed, 1927, p. 134); and Ventrifossa petersoni Alcock, a common archibenthic macrourid of the Indo-Pacific region, of which a damaged specimen, probably misidentified, was once reported from 4391 meters (Weber, 1913, p. 156).

Of the eleven families reported from the ocean floor below 3660 meters, macrourids and brotulids together make up slightly more than half of the species, while macrourids predominate in the number of individuals caught (about forty). However small the numbers seem, it is to be remembered, as Hjort wrote in 1912, that evidence of the scarcity of fishes on the abyssal plain is negative. The number of dredging operations carried on at and below that depth is infinitely less than those executed at higher levels; and the vastness of the area in question is a barrier to an increase in our knowledge and will doubtless continue to be so for many years.

It seems logical to conclude that on the whole life is less concentrated at great depths than elsewhere in the sea (see Murray and Hjort, 1912, p. 419; Krogh, 1934, p. 430; and Marshall, 1954, p. 336), but the deep sea floor may not be as barren as has sometimes been imagined. Knowledge of bottom life will probably be increased through evidence accumulated by use of submarine cameras, underwater television, or even by actual observation. During the only trip ever made to the abyssal plain, sea anemones, numerous "animal holes" and a six-foot shark were observed on bottom at more than 4000 meters, in the Atlantic off Dakar, West Africa (Houot, 1954 , p. 83). On the bottom at a depth of 2347 meters in the Mediterranean, MacLeish (1954, p. 88) reported seeing starfish, sea anemones, many burrows, each"with several paths extending from it, and two fishes "supported several inches above the bottom by slender spines extending from their tails and fins. They too were immobile."

The greatest depth from which any fish is known was recently recorded by a Russian expedition, which captured a new species of the family Liparidae in the northern Pacific at 7200 meters

${ }_{1}^{1}$ The photograph and drawing published by MacLeish suggested that these fishes were Benthosaurus and a later and clearer photograph taken in the same area at a depth of 2132 meters (Edgerton, 1955 , p. 536) virtually proves it. These photographs are intensely interesting, especially since Benthosaurus has not been reported from the Mediterranean. Edgerton also photographed a fish that he called Halargyreus, probably the species listed here as Lepidion lepidion (p. 158). 
(Zenkevich, Birschstein and Beliaev, 1954, p. 67). The only other fish known from below 7000 meters is a specimen of Bassogigas trawled by the Galathea in 7130 meters in the Sunda Trench, which is in the Indian Ocean off Java (Perlman, 1952, p. 66; Bruun, 1953, pp. 261, 262, fig.; 1955, pl. 7). Known below 6000 meters are Grimaldichthys profundissimus (p. 218) and a new genus and species of the family Liparidae taken by the Galathea from the Kermadec Deep in 6660 meters (Bruun, 1953, in litt.; 1955, pl. 6).

\section{DISTINCTIVE CHARACTER OF DEEP-ABYSSAL FAUNA}

About 260 benthic species have been reported from below a depth of about 2000 meters. They belong to 111 genera and thirty-five families. Even should some forms prove to be pelagic there is a considerable increase in number at these lesser depths over those found on the comparatively unknown abyssal plain. The representation is still poor when compared to the more widely explored upper regions, yet individual hauls from these depths have at times yielded large numbers of diverse animal forms, and a further increase in number may be expected with further exploration.

Twenty-three of the families are entirely or predominantly composed of deep-sea fishes, and nine (Scylliorhinidae, Squalidae, Gadidae, Acropomatidae, Zoarcidae, Brotulidae, Cottidae, Liparidae and Ogocephalidae) exhibit a wide bathymetric range, containing numerous shallow-water forms and even, in two cases, fresh-water forms, in addition to many deep-sea species. Only three of the thirty-five benthic families represented in deep-abyssal waters are typical of coastal areas-Rajidae, Congridae and Parapercidae-and none of these is without specialized deep-water genera.

Brotulids predominate in number of genera, macrourids in number of individuals. The families Alepocephalidae, Zoarcidae and Liparidae are also well represented.

Most of the species found exclusively below 2000 meters are known from only a few specimens. Only about forty-three may with any degree of certainty be thought to occur normally in deepabyssal waters and many of the species listed are doubtless only rare or accidental at these depths. However, a critical examination of the records of deep-abyssal genera proves that only Raja is common in surface waters and that almost none of the other genera occur above depths of about $200-400$ meters. It is evident that the deep-abyssal fish fauna is more distinct than the fauna of the upper deep-sea regions. 


\section{ENDEMISM IN DEEP-ABYSSAL WATERS}

In the present discussion geographical divisions are necessarily loosely defined and intended as a convenience. Insufficient information prohibits definition of zoogeographical regions in very deep water, with the possible exception of the Arctic, Antarctic and eastern Pacific areas. The deep-abyssal fish fauna is still comparatively unknown and some of the barriers to dispersal found in littoral and sublittoral zones are lacking in deep-abyssal waters, the various faunal areas thus actually being of wider extent and with less distinct boundaries. After a survey of all abyssal animal forms, Ekman (1953) divided the deep sea into four main areas: Atlantic, Indo-pan-Pacific, Antarctic and Arctic. There is little doubt that the two latter regions are characterized by distinct fish faunas but the other two divisions may not prove tenable. The deep-abyssal faunas of the Atlantic, Indian, and Pacific oceans are tied together by several widely distributed benthic species, whose number will undoubtedly be augmented in the future. However, even these forms will probably show regional variations similar to those found among cosmopolitan bathypelagic fishes such as species of Chauliodus, Stomias, and Nessorhamphus, as discovered in recent years through studies of the abundant material collected by the various Dana expeditions.

Only two families are confined to one area, Bathydraconidae (Antarctic) and Derepodichthyidae (northeastern Pacific).

There are few endemic genera and almost all of these are monotypic, usually with very few examples known. The only nonmonotypic genera are Bathydraco Günther (Antarctic, five species), Bothrocaropsis Garman (eastern Pacific, three species), and Aulastomatomorpha Alcock (north Indian Ocean, two species, three specimens).

The following monotypic genera are endemic.

\section{ARC'TIC}

Liparidae: Rhodichthys Collett

\section{EASTERN ATLANTIC}

Alepocephalidae: Einara Parr, ${ }^{1}$ Bellocia Parr

Searsiidae: Barbantus Parr ${ }^{1}$

Macrouridae: Echinomacrurus Roule

Zoarcidae: Pachycara Zugmayer

Brotulidae: Leucochlamys Zugmayer

${ }^{1}$ Perhaps pelagic. 


\section{WESTERN ATLANTIC}

Stephanoberycidae: Stephanoberyx Gill

\section{EASTERN PACIFIC}

Alepocephalidae: Brunichthys Parr

Searsiidae: Mirorictus Parr, Pellisolus Parr

Derepodichthyidae: Derepodichthys Gilbert

Brotulidae: Leucicorus Garman, Sciadonus Garman

\section{SOUTHEASTERN PAGIFIC}

Parapercidae: Macrias Gill and Townsend

\section{NORTH PACIFIC}

Liparidae: Acantholiparis Gilbert and Burke

MID-PACIFIC

Stephanoberycidae: Malacosarcus Günther

\section{WESTERN PACIFIC}

Brotulidae: Alcockia Goode and Bean, Mastigopterus Smith and Radeliffe, Enchelybrotula Smith and Radcliffe, Typhlonus Günther

\section{NORTH INDIAN}

Congridae: Promyllantor Alcock

Acropomatidae: Brephostoma Alcock

Brotulidae: Tauredophidium Alcock

Thus only twenty-seven (ca. 25 per cent) of the 111 deep-abyssal genera are geographically endemic. Of these the family Brotulidae contains the largest number (eight), followed by the Alepocephalidae and Searsiidae, with seven; Stephanoberycidae, Zoarcidae and Liparidae, each with two; and the following families, each with one: Congridae, Macrouridae, Acropomatidae, Parapercidae, Bathydraconidae and Derepodichthyidae.

\section{ENDEMISM OF SPECIES}

With few exceptions, deep-abyssal species confined to a single area are either known from too few examples for evaluation (and some formerly belonging in this category have since turned up in a far distant locality) or are eurybathic species whose centers of distribution are in much shallower strata, such as Nezumia sclerorhynchus and Oxygadus labiatus. Thus, although a survey reveals a considerable amount of endemism, it is based largely upon species 
that have been taken very rarely, and their endemism must be held in doubt until further investigations confirm the fact that they are limited to one area. Many, or even most, of these rare forms may some day be found elsewhere in deep water. It must be kept in mind that many parts of the oceans are still comparatively little explored, including the southern seas generally and vast areas in the central parts of the Pacific and Atlantic oceans.

Of the comparatively well-represented benthic families, the Liparidae shows the largest percentage of endemic species, twelve of the thirteen deep-abyssal forms (about 92 per cent) being confined to one region. About 87.5 per cent of the zoarcids are endemic, 85 per cent of the brotulids, 72 per cent of the alepocephalids, 67.5 per cent of the macrourids, and 50 per cent of the halosaurids.

Except for the polar regions, the greatest amount of endemism among species is found in the eastern Pacific. Within the limits of our knowledge the region is fairly well defined abyssally in regard to fishes. Fifty-six of the sixty-six deep-abyssal species (85 per cent) are endemic, and of the remaining ten, four are found elsewhere only in the adjoining north Pacific region. All but five of the endemic forms are known from only a few specimens each, however, and of these only one, Bassogigas digittatus, is primarily deep-abyssal. The other four, Lionurus liolepis, Melanostigma pammelas, Dicrolene filamentosa and $D$. nigra, are apparently only accidental below 2000 meters.

Of the six non-endemic eastern Pacific species (excluding those found elsewhere only in the north Pacific), Hemimacrurus acrolepis reaches across the north Pacific to Japan; Antimora rostrata is cosmopolitan; Ilyophis brunneus and Bathysaurus mollis are found in widely separated areas; Bathylaco nigricans is known also from the western Atlantic; and Mixonus caudalis is found in the Indian Ocean. There is little indication of relationship between species of the eastern and western Pacific regions.

In the eastern Atlantic, including the one Mediterranean species, there are about thirty-six endemic species (about 40 per cent). Two of these, Rouleina attritus and Bassogigas crassus, also occur normally below 2000 meters, while five of them have their centers of distribution at higher levels. The other endemic species are known from only one to four specimens each.

The western Pacific, including the southern portion, has twentytwo endemic species (about 52.5 per cent), only one of them, Nezumia parvipes, being more than accidental below 2000 meters. 
The north Pacific has eleven endemic forms (58 per cent), all known from only a few specimens except Macrourus cinereus, which is a straggler in the deep-abyssal zone.

Of the nineteen species (ca. 57.5 per cent) endemic in the northern Indian Ocean, Bassozetus glutinosus and Porogadus trichiurus are probably also common below 2000 meters, while Macrourus woodmasoni has a higher center of distribution.

In the western Atlantic there are about fifteen species (22.8 per cent) not found elsewhere; two, Lycodes atlanticus and Lycodonus mirabilis, are known principally in deep-abyssal waters; one, Gaidropsarus ensis, is perhaps deep-abyssal in the southern part of its range; one, Stephanoberyx monae, has its center of distribution in shallower water; and the remainder are known from too few specimens for evaluation.

In the Arctic region, characterized by negative temperatures, five of the seven species are endemic, Rhodichthys regina being also known from several deep-abyssal captures.

The seven Antarctic species are all known from only a few specimens but it may be significant that the two non-endemic forms are found elsewhere only in neighboring south Atlantic waters.

The three species found nowhere but in the central Pacific, the five endemic South African forms, and the two from the southeastern Pacific are also represented by only one or a few specimens each.

Not one deep-abyssal species has been taken exclusively in the central Atlantic. All forms found there have been caught also in the eastern and/or western Atlantic except Bassozetus compressus, which has been reported from the mid-Atlantic and the western Pacific.

As pointed out by Ekman (1953, p. 305) the Mediterranean Sea possesses an "archibenthal-abyssal" fauna, not a true abyssal one. Only three species ${ }^{1}$ have been reported with certainty from below 2000 meters in the Mediterranean: Centroscymnus coelolepis, Lepidion lepidion, and Chalinura mediterranea. The last-named species is endemic but is closely related to the eastern Atlantic C. murrayi. Only five other deep-abyssal fishes have been reported from the Mediterranean (but not below 2000 meters): Alepocephalus rostratus, Bathypterois dubius, Hymenocephalus italicus, Nezumia aequalis and $N$. sclerorhynchus. All are more or less eurybathic forms.

${ }^{1}$ Four, including the one seen and photographed (p. 81). 
It will be noted in going through the list of species that many of them, although they are known from only a few specimens, are found in widely separated areas; such are Aldrovandia phalacra, which is known from the eastern Atlantic and Indian oceans, and Bathysaurus mollis, known from the eastern Atlantic and also from the eastern and western parts of the Pacific.

The foregoing distributional analysis, which shows that families and genera exhibit very little endemism in deep-abyssal waters, suggests that time is the greatest barrier to the dispersal of deepabyssal fishes. Final analysis of the endemism present on the species level will have to be determined by future investigations; material at the present time is far too inadequate. It will be especially interesting to learn if the present apparent affinity, on the species level, of the deep-abyssal fish fauna of the waters around South Africa to that of the northern Atlantic is based only upon the fact that the Atlantic fauna is better known than that of similar depths in the Indian Ocean; or whether the pathway between South Africa and the Atlantic is actually an easier one to travel than routes between South Africa and other areas in the Indian Ocean.

Of the twenty-one deep-abyssal species found in South African waters five are endemic and fifteen occur also in the Atlantic, while only five are common to the Indian Ocean and only seven to the Pacific. It seems fairly certain that some fishes have spread between the Indian and north Atlantic oceans via South Africa (cf. Bathysaurus ferox, Aldrovandia macrochir, Venefica proboscidea). Communication routes throughout the Atlantic seem to be relatively open, while distances in the Pacific are apparently not so easily bridged.

Another question of great interest is the apparent isolation, faunistically, of the deep-abyssal eastern Pacific region in respect to fish species. Vast stretches of barren areas such as those found in some parts of the Pacific may slow the spreading of many benthic species, thus allowing time for the development of an endemic fauna in the eastern section. However, no conclusions can be reached on the relative composition of this fauna, as practically no bottom fishing has been carried on in deep-abyssal waters in this region since the Albatross expeditions of the late nineteenth and early twentieth centuries. Its relationships on the basis of present knowledge are about equally divided between the Atlantic and other sections of the Pacific, although, if the species common to the adjoining north Pacific area are excluded, the eastern Pacific fish 
fauna, in regard to species, is closer to that of the north Atlantic than to any other.

Endemic species characterize a region. It is through widespread forms that paths of dispersal may be studied. At present only a few such species are known but even these few serve to emphasize the effectiveness of barriers isolating the Arctic and Antarctic deepabyssal, as well as that of the Mediterranean. Antimora rostrata, which has spread farther than any other known benthic deep-abyssal species, may be expected to turn up in the virtually unexplored southeastern Pacific, where it is so far unknown. It is otherwise absent only from the northern Indian Ocean, the Mediterranean, and the polar regions. Several species show a distributional pattern suggesting a minimum of barriers to dispersal in southern seas, for example, Bathysauropsis gracilis, Nematonurus armatus, and Lionurus filicauda.

\section{THE BATHYPELAGIC FISHES}

Of the many bathypelagic (as contrasted with the benthic) fishes known to science, not one species can certainly be said to inhabit waters deeper than 3660 meters. Only a few are known from enough individuals to warrant their inclusion in the present discussion, and of these only the following seven seem to be more common below than above 2000 meters: Cyclothone obscura, Gonostoma bathyphilum, Serrivomer parabeani, Cyema atrum, Melamphaes nigrescens, Atrophacanthus danae, and Edriolychnus schmidti. Records of Eurypharynx pelecanoides are almost equally divided above and below, its principal area of occurrence being between about 1400 and 2500 meters.

Since all oceanic animal life is presumably dependent upon the production of plants in the upper lighted zones of the sea, bathypelagic organisms would be expected to thin out quantitatively in waters below the plant-producing zone. However, it need not follow that life is lacking in the deepest intermediate layers. The daily vertical migrations undertaken by some of the small pelagic and bathypelagic organisms, including fishes, that belong near the bottom of the marine food pyramid, are a factor in the ecology of deep-abyssal waters that has not always received enough emphasis. ${ }^{1}$ Some bathypelagic fishes, for example, certain species of the families Myctophidae and Gonostomatidae, which occur in enormous num-

${ }^{1}$ Marshall (1954, pp. 157-181, 334-349) has given a remarkably clear picture of the vertical distribution of bathypelagic life. 
bers, travel upward from the depths at night to feed near, or nearer, the surface. When they return to their respective deeper levels by day they serve as food for the larger organisms that may be confined to those depths. It is also quite probable that in spite of the emphasis given to the enormous quantity of life produced in the upper sea layers, the volume has been underestimated rather than exaggerated and there are perhaps no intermediate regions incapable of supporting abundant life in areas where surface organisms are plentiful.

It seems particularly significant that Houot (1955, p. 249), who has participated in numerous bathyscaphe dives in the Mediterranean and the Atlantic to depths as great as 4000 meters, judged the density of the plankton to be about equal from 150-250 meters to within two or three meters of the bottom.

The presence of dense plankton in abyssal and deep-abyssal waters would account for the food supply of benthic animal communities and would increase the importance and complexity of the vertical migrations of bathypelagic organisms. Regarding the density of organisms in intermediate waters, Cousteau (1954, p. 78; 1954, personal communication), in his descent to the bottom at a depth of 1200 meters in the Mediterranean off Toulon, was forcibly impressed with the fact that pelagic life increased, relative to depth, both in volume and in the size of the individual. Beebe (1934, pp. 700, 703), who was lowered in the bathysphere to 900 meters, off Bermuda, reported "the total number of creatures unbelievable" and was astounded at the number of large animals present in deep water. MacLeish (1954, p. 88), like Beebe and Cousteau, was impressed with the amount of plankton: "The plankton here [350-1400 m.] is so dense that it looks like a fine driving snow rushing upward. The water is like a soup. It seems to be half composed of living things."

A few (17) bathypelagic fishes are included here as a part of the fauna of deep-abyssal waters because there seems to be enough evidence to show that their normal range extends to these depths. There are, of course, other little-known forms that may prove to be inhabitants of the deep-abyssal zone. All of the species taken exclusively in nets fished below 2000 meters are known from only one or two specimens each and so cannot be considered here. There is the ever-present possibility that the species are "rare" merely because of their agility in escaping the slowly moving nets; or because the few specimens caught may have entered open nets at 
higher levels. Certain bathypelagic forms, including those mentioned above, will be discussed in the list of species along with the benthic forms known or thought to inhabit the sea floor below 2000 meters. All species of the order Ceratioidea have been listed, whether known to be deep-abyssal or not, partly for the reasons given on page 232 and partly because Bertelsen's studies (1951), based on the Dana material, present an evaluation of the group to which only scattered additions are to be expected in the near future.

The following species have been taken in closing nets hauled in deep-abyssal intermediate waters but have been omitted from the list either because they have been captured so rarely that the records may be accidental or because they are common in shallower depths (see also families Chiasmodontidae, p. 193 and Melamphaidae, p. 191).

Dolichopteryx sp., one specimen taken by the Discovery in the Atlantic (ca. $5^{\circ} \mathrm{N} ., 17^{\circ} \mathrm{W}$.) at a depth of $2500-2700$ meters. Norman $(1930$, p. 271$)$ identified this example with $D$. longipes Vaillant, but Parr (1937, p. 34) considered it to represent another species, perhaps $D$. binocularis Beebe.

Electrona parallela Lönnberg, two specimens, also taken by the Discorery in the Atlantic, off South Africa, 2500-2000 meters. The species is otherwise known from only seven specimens, two in 350-400, one in 1000 and four in 2000 and 2500 meters.

Diogenichthys laternatus Garman, four specimens caught by the Discovery off South Africa, 2500-2000 meters. The species is an abundant one and cosmopolitan in distribution between $34^{\circ} \mathrm{N}$. and $34^{\circ} \mathrm{S}$.

It is well to note here that lack of data on the habits of deep-sea fishes often makes it impossible to determine whether a species is primarily benthic or bathypelagic; and also that some forms probably belong in both categories. Some benthic species rise to intermediate waters at spawning time, while there are undoubtedly bathypelagic species that seek bottom for spawning. It is also quite possible that some bathypelagic fishes approach bottom in pursuit or in search of prey as well as that various benthic forms leave the sea floor for the same reasons. Then, too, bathypelagic species undergoing daily vertical migrations live normally over great depths but where such depths adjoin shallower areas it is logical to find them on or near the bottom at lesser depths (see discussion of Cyclothone microdon, p. 125). There is also a growing belief, first suggested by Dr. August Krogh in June, 1933, at a symposium on the conditions of existence of aquatic animals (Krogh, 1934, p. 436), that there exists a benthopelagic fauna, which might well include a num- 
ber of fishes not actually living upon the bottom and yet not entirely independent of it.

Monod's observations (1954, p. 1951) on bottom in 1400 meters off Dakar (eastern Atlantic) in some respects change the current concept of bathypelagic life, for he saw typical bathypelagic organisms (medusae, red shrimps, chaetognaths) actually close to or in contact with the bottom. The fact that both micro- and macroplankton extend all the way to the sea floor (at least in some regions) explains several benthic captures of typically bathypelagic fishes, for example, Avocettina infans (p. 150).

The phenomenon of ontogenetic vertical migration, undertaken by members of diverse groups of bathypelagic (and perhaps benthic) fishes, also considerably complicates "placing" a species at any particular depth. Most ceratioids, for example, hatch from the egg in depths of 50 to 200 meters and make a rapid descent, upon metamorphosis, to about 2000-2500 meters, at which depth are also found most of the mature females and the metamorphosing or metamorphosed males. After metamorphosis, females are most frequently found between 1500 and 2000 meters, but upon reaching maturity they again return to the 2000-2500 meter level (Bertelsen, 1951). Actually, therefore, the normal habitat of these fishes is 50-2500 meters, although some species are undoubtedly truly deepabyssal, since the males seem to keep to the greater depths whether or not they become parasitic upon the females. Known specimens of most ceratioid species are far too few and their life-histories too little known to allow an accurate estimate of their maximum occurrence, as adults, at any particular level; and although the exaggerated sexual dimorphism found in these fishes further obscures an understanding of their ecology, groups with seemingly simpler life-histories are as little understood.

A certain amount of conjecture is therefore involved in separating deep-sea fishes into benthic and bathypelagic groups. Conjecture plays a major part in almost any study concerned with deep-sea life. Among various impressions gained from a fairly thorough survey of the literature dealing with deep-sea fishes, irrelevant to the present work, is the fact that many species, particularly the "rare" ones, are young, some even larval, at an unusually large size. One of the theories advanced to explain this phenomenon is that low temperature and other physical factors of the environment cause deep-sea fishes to undergo a protracted juvenile period and even, occasionally, to reach the breeding stage before acquiring all adult characters. It has been suggested that some species retain 
their larval forms throughout life, paralleling the phenomenon of neoteny in the Amphibia. It is possible that in many cases only the young have been caught, the larger adult forms escaping the nets; in this event it would not be at all surprising to find some truly gigantic species in deep water.

\section{CONCLUSION}

The original purpose of this report was to make available as many references as possible to the known fish fauna of deep-abyssal waters and to present a summary of the information gleaned through such a survey, in the hope of adding to our knowledge of the zoogeography of the area. During the course of the work it has become apparent that a presentation of known facts serves chiefly to accentuate the many remaining unknown. Figures and percentages offered here are almost meaningless when one realizes how few bottom hauls have been made in deep-abyssal waters. In the boreal north Pacific, for example, only nineteen bottom hauls have been made deeper than 2000 meters, yielding twenty specimens of fishes. The number of such hauls made in Arctic and Antarctic waters is probably still less. Even in the most extensively explored area, the eastern Atlantic, only a few hundred such trials have been made and by no means all of these have been successful; for instance, of 137 deep-abyssal fishing efforts undertaken by the Prince of Monaco during many expeditions in the Atlantic between 1888 and 1914, the gear was lost in twenty-eight cases, in sixteen it failed to reach bottom, and in several instances it came to the surface virtually empty. In other words, about a third of the attempts were unsuccessful.

Negative results are not, however, valueless, and among other things they serve to emphasize the importance of such expeditions as that undertaken by the Galathe ${ }^{1}$ and the fact that a clear pic-

${ }^{1}$ A brief indication of future changes in the distribution of deep-abyssal fishes may be seen in comparing with the text the following data seen (1953) with a few of the Galathea fishes on exhibition at the Zoological Museum in Copenhagen.

Bathysaurus mollis, Indian Ocean off East Africa, 4060 meters.

Benthosaurus, Indian Ocean, Mozambique Channel, 3485 meters.

Bathymicrops (Bruun, 1955, pl. 5), north of Madagascar, 4820 meters; Indian Ocean off South Africa, 4360 meters; northeast of New Zealand, 5850 meters.

Macrouroides inflaticeps, Madagascar-Mombasa, 4820 meters.

A phyonus, off Durban, 4360 meters.

Bassogigas, Java Deep, 7150 meters.

Acanthonus armatus, Gulf of Guinea off San Tomé, 2550 meters.

Liparidae, gen. and sp. nov., Kermadec Deep, 6000 meters. 
ture of life at the greatest depths of the sea depends upon many more such investigations.

\section{NOTE}

In May, 1955, the United States Fish and Wildlife Service Research Vessel Oregon trawled in the Gulf of Mexico (2104-2194 meters) a number of benthic fishes, which will be described in detail at a future date. Some of these new records are reported here and, excepting specimens of Gadomus longifilis and Melanocetus murrayi from earlier Oregon hauls, are the only new records included. See Alepocephalus productus, p. 104; Conocara murrayi, p. 115; Benthosaurus grallator, p. 131; Bathypterois phenax, p. 133 (first deep-abyssal capture); Ipnops murrayi, p. 136; Venefica procera, p. 141 (first deep-abyssal capture); Synaphobranchus kaupi, p. 145; Bassozetus normalis, p. 208; Porogadus miles, p. 211; and Porogadus subarmatus, p. 213.

EDITORIAL NOTE

[The practice of omitting parentheses from authors' names when parentheses are called for by the International Rules of Nomenclature was established for the zoological publications of Chicago Natural History Museum by the late Wilfred Hudson Osgood as a protest against what seemed to him an "outworn nomenclatural practice." We are conforming to this practice in concurrence with Dr. Osgood's argument.-KARL P. ScHMIDT]

\section{ACKNOWLEDGMENTS}

I am greatly indebted to Dr. Karl P. Schmidt, Dr. Anton F. Bruun, Dr. Robert F. Inger, Dr. Norman Willimovsky, and Dr. George S. Myers for advice and encouragement; to Dr. Robert R. Harry for the use of his unpublished manuscript on the classification of iniomous fishes; to $\mathrm{Mr}$. Dwight Davis for the translation of Russian passages; to Mrs. Meta Howell and her assistants for their untiring efforts in locating rare books; and to Mr. Loren P. Woods, Dr. Leonard Schultz, and Mrs. Myvanwy Dick for information about various specimens in the collections of the United States National Museum and in the Museum of Comparative Zoology at Harvard University. Miss Lillian Ross, Associate Editor, has been especially helpful throughout the preparation and revision of this paper, and she has my sincere gratitude. 


\section{Synonymies ${ }^{1}$ and Distribution}

\section{ELASMOBRANCHII}

Of the relatively few deep-sea elasmobranchs, none has ever been taken below 3660 meters $^{2}$ and very few are recorded deeper than 2000 meters. The widely distributed species, Hexanchus griseus Bonnaterre, has been reported once from deep-abyssal waters (Bragança, 1904, p. 28), off Portugal in 1875 meters, but it is unlikely that the shark was actually caught at that depth. It has been taken frequently in much shallower water and has been seen on the surface at night (Fraser-Brunner, 1935, p. 319). Isistius brasiliensis Quoy and Gaimard, another far-ranging pelagic shark, has been recorded twice from deep-abyssal waters but, like Hexanchus griseus, has frequently been caught in much shallower water as well as on the surface at night, and the specimens reported from 1829 and 2488 meters (Bigelow and Schroeder, 1948, p. 509) may well have entered the nets in transit.

\section{Family SCYLLIORHINIDAE}

Although this family contains numerous deep-sea species, only one has been reported from the region under consideration.

\section{Apristurus indicus Brauer}

Scylliorhinus indicus Brauer, 1906, Wiss. Ergebn. Deutschen Tiefsee Exp. Valdivia, 15, (1), p. 8, pl. 14, fig. 1 -Indian Ocean, ca. $2^{\circ} \mathrm{N} ., 47^{\circ}$ E. and ca. $13^{\circ} \mathrm{N} ., 47^{\circ}$ E., 1289 and 1840 meters, type locality not designated.

Scylliorhinus indicus Regan, 1908, Ann. Mag. Nat. Hist., (8), 1: 459; Lloyd, 1909, Mem. Indian Mus., 2: 139; Holt and Byrne, 1909, Ann. Mag. Nat. Hist., (8), 3: 279; Koefoed, 1927, Rep. Sci. Res. M. Sars No. Atl. Deepsea Exp. 1910, 4, (1), p. 19.

Apristurus indicus Garman, 1913, Mem. Mus. Comp. Zool., 36: 97; Bigelow and Schroeder, 1944, Proc. New England Zool. Club, 23: 22; 1948, Mem. Sears Found. Mar. Res., 1, (1), p. 221.

Scylliorhinus (Halaelurus) indicus Norman, 1939, Sci. Rep. John Murray Exp. 1933-34, 7, (1), p. 10; Misra, 1950, Rec. Indian Mus., 45: 12; 1952, op. cit., 49: 102, fig. 4, $a$.

Pentanchus indicus Fowler, 1941, Bull. U. S. Nat. Mus., 100, (13), p. 60.

Deep-abyssal record.-Gulf of Aden, 1840 meters, one specimen.

1 References marked with an asterisk have not been seen by the author.

${ }^{2}$ However, one was seen at a depth of about 4000 meters (p. 81). 
Distribution.-Indian Ocean off Somaliland, in the Gulf of Aden and the Gulf of Oman, one deep-abyssal record and four specimens in 1061-1289 meters. Eastern Atlantic, one young specimen off southwest Ireland in 1225-1409 meters. Probably accidental in deep-abyssal waters.

Length.-132-336 mm.

\section{Family SQUALIDAE}

The only elasmobranch known to inhabit deep-abyssal waters more than accidentally is a squaloid shark, Centroscymnus coelolepis. Etmopterus princeps is probably only accidental below 2000 meters, while $E$. spinax Linnaeus and $E$. pusillus Lowe, archibenthic species that are pelagic or semi-pelagic with one deep-abyssal record each, are not included in the present discussion. Numerous species of the family are found in shallower deep-sea areas.

\section{Etmopterus princeps Collett}

Etmopterus princeps Collett, 1904, Vidensk. Selsk. Forh., 1904, (9), p. 3Faroe Channel, 1100 meters.

Etmopterus princeps Collett, 1905, Rep. Norwegian Fish. Mar. Invest., 2, (3), p. 29, pl. 1, figs. 1, 2; Garman, 1913, Mem. Mus. Comp. Zool., 36: 222; Bigelow and Schroeder, 1948, Mem. Sears Found. Mar. Res., 1, (1), p. 488; 1954, Bull. Mus. Comp. Zool., 112: 46; Bigelow, Schroeder and Springer, 1953, op. cit., 109: 241, 246, fig. 8.

Spinax princeps Regan, 1908, Ann. Mag. Nat. Hist., (8), 2: 44; Koefoed, 1927, Rep. Sci. Res. M. Sars No. Atl. Deep-sea Exp. 1910, 4, (1), p. 21, pl. 3, fig. 4 .

Spinax (Etmopterus) princeps Murray and Hjort, 1912, Depths of Ocean, p. 392 .

Deep-abyssal record.--Eastern Atlantic, ca. $35^{\circ}$ N., $8^{\circ}$ W., 2055 meters, one specimen.

Distribution.--Eastern Atlantic, one deep-abyssal record and four specimens off the Faroe Islands and in the Faroe-Shetland Channel in 1100 and 750 meters. Western Atlantic from off Nova Scotia to southern New England, fifty-four specimens, 567-951 meters. Apparently accidental in deep-abyssal waters.

Length. $-190-728 \mathrm{~mm}$.

\section{Centrophorus squamosus Bonnaterre}

Squalus squamosus Bonnaterre, *1788, Tabl. Encyc. Meth. Ichth., p. 12 -Atlantic off Portugal. 
Squalus squamosus Gmelin, *1788, Syst. Nat. Linn., ed. 13, 1: 1502.

Lepidorhinus squamosus Bonaparte, *1838, Nuov. Ann. Sci. Nat., 2: 207; Garman, 1913, Mem. Mus. Comp. Zool., 36: 211; Roule, 1919, Rés. Camp. Sci. Monaco, 52: 118; de Buen, 1931, Bol. Oceanogr. Pesc., Inst. Esp. Oceanogr., 171: 15; 1935, Not. Res. Inst. Esp. Oceanogr., (2), 88: 32; Fowler, 1936, Bull. Amer. Mus. Nat. Hist., 70: 78, fig. 25; FraserBrunner, 1938, Ann. Mag. Nat. Hist., (11), 2: 410; Bertin, 1939, Bull. Mus. Hist. Nat. Paris, 11: 75; Coelho, 1942, Trav. Stat. Biol. Marit. Lisbonne, $46: 8$.

Centrophorus squamosus Müller and Henle, 1841, Syst. Beschr. Plagiost., p. 90, pls. 33, 34; Lowe, 1852, Ann. Mag. Nat. Hist., (2), 10 : 55; Bocage and Capello, 1864, Proc. Zool. Soc. London, 1864: 260; ${ }^{*} 1866$, Peixes Plagiost., 1 : 27, pl. 3, fig. 2; Capello, *1870, Jorn. Sci. Math. Phys. Nat., 2: 144; *1880, Cat. Peixes Portugal, p. 48; Hilgendorf, *1888, Arch. Naturg. (2), 54: 213; Vaillant, 1888, Exp. Sci. Trav. Talis., Poiss., p. 69, pl. 2, fig. 3, and pl. 3, fig. 2; Holt and Calderwood, 1895, Sci. Trans. Roy. Dublin Soc., 5: 372, pl. 43, fig. 1, text fig.; Goode and Bean, 1895, Ocean. Ichth., p. 13; Vieira, *1898, Cat. Peixes Portugal, p. 14; Saemundsson and Jensen, 1899, Vidensk. Medd. Dansk naturh. Foren., 1899: 409, pl. 3; Bragança, 1902, Bull. Camp. Sci. Amelia, 1: 76; 1904, Res. Invest. Sci. Amelia, Ichth., 2: 74, 106; Collett, 1905, Rep. Norwegian Fish. Mar. Invest., 2, (3), p. 19; Regan, 1908, Ann. Mag. Nat. Hist., (8), 2: 49; Holt and Byrne, 1910, Rep. Fish. Ireland 1908, Sci. Invest., 5: 1; Seabra, *1911, Bull. Soc. Portug. Sci. Nat., 5: 198; Murray and Hjort, 1912, Depths of Ocean, p. 392, fig. 256; Roule, 1912, Bull. Inst. Océanogr.

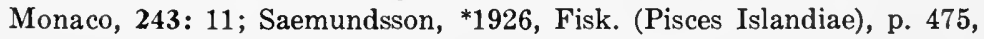
fig. $250 ;{ }^{*} 1927$, Syn. Fishes Iceland, p. 51; 1932, Faune Ichth. Atl. Nord, 10, fig.; 1949, Zool. Iceland, 4, (72), p. 121 (further refs., Iceland); Hickling, 1928, Ann. Mag. Nat. Hist., (10), 2: 196; Lozano Rey, 1928, Fauna Ibérica, Peces, 1: 440, figs. 144, 145; Noronha and Sarmento, *1934, Peixes Madeira, p. 134; Fraser-Brunner, 1935, Proc. Roy. Irish Acad., B42: 320; Nobre, 1935, Fauna Marinha Portugal, 1: 452; Ehrenbaum, ${ }^{*} 1936$, Naturg. wirtsch. Bedeut. Seefische Nordeur., p. 287; Bigelow and Schroeder, 1948, Mem. Sears Found. Mar. Res., 1 : 451; Bigelow, Schroeder and Springer, 1953, Bull. Mus. Comp. Zool., 109: 225.

Machephilus dumerilli Johnson, 1867, Proc. Zool. Soc. London, p. 713.

Centrophorus dumerili Günther, 1870, Cat. Fishes Brit. Mus., 8: 422; Goode and Bean, 1895, Ocean. Ichth., p. 13; Regan, 1908, Ann. Mag. Nat. Hist., (8), $2: 49$.

Centrophorus squamosus var. dumerili Vaillant, 1888, Exp. Sci. Trav. Talis., Poiss., p. 69, pl. 3, fig. 3.

Deep-abyssal record.-Eastern Atlantic off Portugal, 1875 meters, one specimen.

Distribution.-Eastern Atlantic from Iceland and the North Sea to Portugal, Madeira and the Azores, common between about 400 and 1500 meters, one deep-abyssal record.

Length. $-260-1500 \mathrm{~mm}$. 
Remarks.-Hickling (1928, p. 196) believed the species to be pelagic. It probably does not reach deep-abyssal waters and is perhaps accidental even below 1000 meters. The young specimen reported by Roule (1912) from the Mediterranean near Monaco in 2368 meters has been shown to be a young Centroscymnus coelolepis by Tortonese (1952, p. 387), who suggested that the specimens from Nice and Monaco recorded by Carus (1893, p. 503) are probably referable to that species also. C. squamosus probably does not inhabit the Mediterranean.

Centroscymnus coelolepis Bocage and Capello. Table 1.

Centroscymnus coelolepis Bocage and Capello, 1864, Proc. Zool. Soc. London, p. 263, fig. 4-Atlantic off Portugal.

Centroscymnus coelolepis Capello, *1870, Jorn. Sci. Math. Phys. Nat., 2: 144; de Buen, 1935, Not. Res. Inst. Esp. Oceanogr., (2), 88: 31; Ehrenbaum, *1936, Naturg. wirtsch. Bedeut. Seefische Nordeur., p. 287; Coelho, 1942, Trav. Stat. Biol. Marit. Lisbonne, 46: 10; Bigelow and Schroeder, 1948, Mem. Sears Found. Mar. Res., 1, (1), p. 494, figs. 94, 95 (complete synonymy); 1953, Fish. Bull., Fish Wildlife Serv., 53, (74), p. 52, fig. 20; 1954, Bull. Mus. Comp. Zool., 112: 47, fig. 2; Tortonese, 1950, Arch. Zool. Ital., 35: 132; 1952, op. cit., 37: 386, fig. 1; Brandes, Kotthaus and Krefft, 1954, Cons. Perm. Int. Expl. Mer, Ann. Biol., 10 : 45.

Centrophorus squamosus Carus, 1893, Prodr. Faunae Medit., 2: 503; Neuville, 1897, Bull. Mus. Hist. Nat. Paris, 3: 55; Roule, 1912, Bull. Inst. Océanogr. Monaco, 243.

Centrophorus coelolepis Saemundsson, 1949, Zool. Iceland, 4, (72), p. 122 (further refs., Iceland).

Scymnodon melas Bigelow, Schroeder and Springer, 1953, Bull. Mus. Comp. Zool., 109: 233, fig. 5.

Deep-abyssal records.-Western Mediterranean, 2330-2718 meters, thirty-five specimens, most of them taken in traps, one to seven per haul.

Eastern Atlantic in the Gulf of Gascony and off the Azores and Portugal, 1875-2620 meters, eighteen specimens.

Distribution.--Eastern Atlantic from Iceland and Faroe Bank to Cape Verde, Madeira and the Azores, center of distribution between 1000 and 2000 meters. Western Mediterranean, center of distribution below 2000 meters. Western Atlantic from off Newfoundland to off New York and Delaware Bay $\left(38^{\circ}-39^{\circ}\right.$ N.), 330 to $1153-1235$ meters.

Length.-To $1140 \mathrm{~mm}$.

Remarks.-C. coelolepis is apparently the deepest-living shark known. It is partially bathypelagic but its relative frequency in traps set on the bottom proves it to be not exclusively so. Roule 
(1919, p. 120) records five trap-catches (twenty-two specimens) from deep-abyssal waters, four in the Mediterranean and one in the Gulf of Gascony.

\section{Family RAJIDAE}

\section{Raja hyperborea Collett}

Raja hyperborea Collett, ${ }^{*} 1878$, Vidensk. Selsk. Forh., 1878, (14), p. 7-west of Spitzbergen, ca. $79^{\circ} \mathrm{N} .5^{\circ}$ E., 839 meters.

Raja hyperborea Knipowitsch, 1926, Trans. Inst. Sci. Explor. North, 27: 59, fig. 16; Hickling, 1928, Ann. Mag. Nat. Hist., (10), 2: 197; Essipov, 1937, Prob. Arctic, Arctic Inst. U.S. S. R., 4: $97 ;{ }^{*} 1937$, Comm. Fishes Barents Sea, p. 19, fig. 8; Saemundsson, 1949, Zool. Iceland, 4, (72), p. 130; Bigelow and Schroeder, 1953, Mem. Sears Found. Mar. Res., 1, (2), p. 206, figs. 43, 44 (complete synonymy); Andriashev, 1954, Fauna S. S. S. R., 53 : 58, figs. 26-23.

Deep-abyssal record.-Arctic Ocean, ca. $69^{\circ}$ N., $7^{\circ}$ W., 2394 meters, one specimen.

Distribution.-Arctic only, off west Greenland from ca. $60^{\circ}-78^{\circ}$ $\mathrm{N}$. and from east Greenland, Spitzbergen and the eastern Barents Sea south to Iceland and the Faroe Channel. Numerous specimens recorded, 183-293 to 1428 meters, with one deep-abyssal record. Probably accidental in deep-abyssal waters.

Length. $-160-860 \mathrm{~mm}$.

Remarks.-According to Essipov (1937, p. 97) the deepest record is 2640 meters.

Raja jenseni Bigelow and Schroeder

Raja jenseni Bigelow and Schroeder, 1950, Bull. Mus. Comp. Zool., 103: 385, pl. 1 -Atlantic off U. S. coast, ca. $39^{\circ}$ N., $71^{\circ}$ W., 1908 meters.

Raja jenseni Bigelow and Schroeder, 1953, Mem. Sears Found. Mar. Res., 1, (2), p. 213, figs. 45, 46 (complete synonymy); 1954, Bull. Mus. Comp. Zool., 112: 60 .

Deep-abyssal records.-Western Atlantic off New England, 2296, 1908 meters, two specimens.

Distribution.-Western Atlantic from off Halifax, Nova Scotia, to off mouth of Delaware Bay, two deep-abyssal specimens and three in $366-1088$ meters.

Length. $-223-850 \mathrm{~mm}$.

\section{Raja fyllae Lütken}

Raja fyllae Lütken, *1888, Vidensk. Medd. Dansk naturh. Foren., 1887: 1, pl. 1-Davis Strait, 147 meters. 
Raja fyllae var. lipacantha Ehrenbaum, 1927, Fische Nord. Ostsee, 12a: 30, fig. 22.

Raja fyllae Fowler, 1936, Bull. Amer. Mus. Nat. Hist., 70 : 109; Essipov, 1937, Prob. Arctic, Arctic Inst. U. S. S. R., 4: 97; Bigelow and Schroeder, 1953, Mem. Sears Found. Mar. Res., 1, (2), p. 194, figs. 39, 40 (complete synonymy); 1954, Bull. Mus. Comp. Zool., 112: 54, fig. 3; Andriashev, 1954, Fauna S. S. S. R., 53: 61, figs. 29, 30.

Deep-abyssal record.-Eastern Atlantic, ca. $35^{\circ}$ N., $8^{\circ}$ W., 2055 meters, one specimen.

Distribution.-North Atlantic and Arctic (but only at positive temperatures according to Jensen, 1948, p. 46) from the Barents Sea and off western Greenland $\left(63^{\circ}-66^{\circ} \mathrm{N}\right.$.) south to ca. $35^{\circ} \mathrm{N} ., 8^{\circ} \mathrm{W}$. in the east and off Nova Scotia and Georges Bank in the west. One deep-abyssal record and numerous specimens reported between $147^{1}$ and 1797 meters. Probably accidental in deep-abyssal waters.

Length. $-72.5-555 \mathrm{~mm}$.

\section{Raja bathyphila Holt and Byrne}

Raja bathyphila Holt and Byrne, 1908, Rep. Fish. Ireland 1906, Sci. Invest., 5: 189 -Atlantic, ca. $51^{\circ} \mathrm{N} ., 12^{\circ} \mathrm{W} ., 1230-1634$ meters.

Raja bathyphila Bigelow and Schroeder, 1953, Mem. Sears Found. Mar. Res., 1, (2), p. 159, figs. 30, 31, a (complete synonymy); 1954, Bull. Mus. Comp. Zool., 112 : 52.

Deep-abyssal records.-Western Atlantic, ca. $41^{\circ} \mathrm{N} ., 65^{\circ} \mathrm{W}$. and ca. $39^{\circ}$ N., $71^{\circ}$ W., 2173,1908 meters, two specimens.

Distribution.-Western Atlantic off the U. S. coast, $36^{\circ}-41^{\circ} \mathrm{N}$., two deep-abyssal specimens and five from five hauls in 1813, 1570, 1527, 896 and 676-823 meters. Eastern Atlantic off southwest Ireland, one specimen, 1230-1634 meters.

Length.-117-463 mm.

\section{Raja microtrachys Osburn and Nichols}

Raja microtrachys Osburn and Nichols, 1916, Bull. Amer. Mus. Nat. Hist., 35: 142 , fig. 1 - off southern California, ca. $32^{\circ}$ N., $116^{\circ} \mathrm{W} ., 1994$ meters (erroneously reported from Guadalupe Island in type description).

Raja microtrachys Townsend and Nichols, 1925, Bull. Amer. Mus. Nat. Hist., 52: 6 .

Deep-abyssal record and distribution.--Eastern Pacific, 1994 meters, one specimen.

Length. $-737 \mathrm{~mm}$.

1 Jensen (1948, p. 44) thought that the shallow depth of capture recorded for the type might have been an error. The shallowest record otherwise is 274 meters. 


\section{Raja badia Garman}

Raja badia Garman, 1899, Mem. Mus. Comp. Zool., 24: 22, pl. 6, figs. 1, $2-$ Pacific off Panama, ca. $7^{\circ} \mathrm{N} ., 79^{\circ} \mathrm{W} ., 2323$ meters.

Raja badia Garman, 1913, Mem. Mus. Comp. Zool., 36: 357; Beebe and

Tee-Van, 1941, Zoologica, 25: 254, fig.

Deep-abyssal record and distribution.-Eastern Pacific, 2323 meters, one specimen.

Length. - $-257 \mathrm{~mm}$.

\section{Raja abyssicola Gilbert}

Raja abyssicola Gilbert, 1896, Rep. U. S. Fish Comm., 1893: 396, pl. 20-

Pacific off the Queen Charlotte Islands.

Raja abyssicola Jordan and Evermann, 1896, Bull. U. S. Nat. Mus., 47: 76;

Garman, 1913, Mem. Mus. Comp. Zool., 36: 344; Clemens and Wilby, 1946, Bull. Fish. Res. Bd. Canada, 68: 67, fig. 25.

Deep-abyssal record and distribution.-Eastern Pacific, 2904 meters, one specimen.

Length. $-1143 \mathrm{~mm}$.

\section{HOLOGEPHALI}

Chimaeroids are principally deep-sea forms but only three species are found in deep-abyssal waters.

\section{FamilycCHIMAERIDAE}

\section{Hydrolagus affinis Capello}

Chimaera affinis Capello, *1868, Jorn. Sci. Math. Phys. Nat., 1: 314, 320, pl. 3-Atlantic off Portugal in deep water.

Chimaera affinis Dean, 1906, Publ. Carnegie Inst. Washington, 32: 7, 13, 111, 112, 122, figs. 90, 101; Holt and Byrne, 1910, Rep. Fish. Ireland 1908, Sci. Invest., 4: 5.

Psychichthys affinis de Buen, 1935, Not. Res. Inst. Esp. Oceanogr., (2), 88: 42 .

Hydrolagus affinis Bigelow and Schroeder, 1952, Breviora, Mus. Comp. Zool., 8: 5; 1953, Mem. Sears Found. Mar. Res., 1, (2), p. 539, fig. 121 (complete synonymy); 1953, Fish. Bull., Fish Wildlife Serv., 53, (74), p. 79, fig. 35; 1954, Bull. Mus. Comp. Zool., 112 : 70.

Deep-abyssal record.-Western Atlantic, ca. $40^{\circ}$ N., $66^{\circ} \mathrm{W}$, 2360 meters, one specimen.

Distribution.-Eastern Atlantic off Portugal in deep water. Western Atlantic from the Grand Banks of Newfoundland to off 
Cape Cod and ca. $39^{\circ} \mathrm{N}$. (?)Gulf of Mexico. ${ }^{1}$ One deep-abyssal specimen and numerous records in 293-1761 meters. Probably accidental in deep-abyssal waters.

Length. - $914-1283 \mathrm{~mm}$.

\section{Hydrolagus purpurescens Gilbert}

Chimaera purpurescens Gilbert, 1905, Bull. U. S. Fish Comm., 23, (2), p. 582, fig. 231 -off the Hawaiian Islands.

Chimaera gilberti Garman, 1911, Mem. Mus. Comp. Zool., 40: 90; Fowler, 1928, Mem. Bishop Mus., 10 : 26.

Psychichthys purpurascens Jordan and Hubbs, 1925, Mem. Carnegie Mus., 10: 117.

Chimaera purpurescens Fowler, 1941, Bull. U. S. Nat. Mus., 100, (13), p. 494. Hydrolagus purpurescens Bigelow and Schroeder, 1953, Mem. Sears Found. Mar. Res., 1, (2), pp. 534, 536.

Deep-abyssal record and distribution.-Pacific off the Hawaiian Islands, 1750-1951 meters, one specimen.

Length. $-900 \mathrm{~mm}$.

\section{Family RHINOCHIMAERIDAE}

Besides the one species discussed below, the only evidence that members of this family inhabit the deep-abyssal zone is the presence in a haul in the Atlantic (ca. $28^{\circ} \mathrm{N} ., 24^{\circ}$ W.), at a depth of 5000 meters, of an egg-capsule membrane (Koefoed, 1927, p. 31). Koefoed stated that it probably belonged to a species of Rhinochimaera.

\section{Harriotta raleighana Goode and Bean}

Harriotta raleighana Goode and Bean, 1895, Proc. U. S. Nat. Mus., 17: 471, pl. 19 -Atlantic off U. S. coast, ca. $39^{\circ}$ N., $70^{\circ}$ W., 1977 meters.

Harriotta raleighana Goode and Bean, 1895, Ocean. Ichth., p. 33, figs. 37-40; Jordan and Evermann, 1896, Bull. U. S. Nat. Mus., 47:96; 1900, op. cit., fig. 42; Garman, 1904, Bull. Mus. Comp. Zool., 41 : 263, pl. 2, figs. 3-5, pl. 4, fig. 1, and pl. 5, figs. 3-9; 1911, Mem. Mus. Comp. Zool., 40:95; Dean, 1906, Publ. Carnegie Inst. Washington, 32: 6, 121, 122, figs. 94, 96; Bean and Weed, 1910, Proc. U. S. Nat. Mus., 37: 662, pl. 38; Murray and Hjort, 1912, Depths of Ocean, pp. 394, 417, figs. 260, 307; Koefoed, 1927, Rep. Sci. Res. M. Sars No. Atl. Deep-sea Exp. 1910, 4, (1), p. 29, pl. 3, figs. 1, 2, text figs. 4-6; Roule and Angel, 1933, Rés. Camp. Sci. Monaco, 86: 75, pl. 4, fig. 34; Fowler, 1936, Bull. Amer. Mus. Nat. Hist., 70 : 145, fig. 60; Roule, 1934, Poiss. Monde Viv. Eaux, 7: 161, 163, pl. 9, text fig.;

${ }^{1}$ Bigelow and Schroeder $(1953$, p. 544) reported that this specimen is now lost and suggested that it might have been $H$. alberti Bigelow and Schroeder, a species known only from the Gulf of Mexico off Pensacola. 
Bigelow and Schroeder, 1953, Mem. Sears Found. Mar. Res., 1, (2), p. 551, figs. 124-126; 1954, Bull. Mus. Comp. Zool., 112: 81, figs. 6, $d, 6, e, 7$.

? sp. Koefoed, 1927, Rep. Sci. Res. M. Sars No. Atl. Deep-sea Exp. 1910, 4, (1), p. 31, fig. 7 .

Deep-abyssal records.-Eastern Atlantic, ca. $27^{\circ}$ N., $14^{\circ}$ W., 2603 meters, one specimen.

Western Atlantic, 1977 meters, the type.

Eastern Atlantic, ca. $57^{\circ} \mathrm{N} ., 11^{\circ} \mathrm{W} ., 1853$ meters, two specimens.

Distribution.-Eastern Atlantic near the Canary Islands and west of Scotland, two deep-abyssal records. Western Atlantic, $36^{\circ}-42^{\circ} \mathrm{N}$., one deep-abyssal record and forty-four specimens taken between 530-622 and 1813 meters, principally in 700-1000 meters.

Length.-120-1025 mm.

Remarks.-An egg-capsule possibly belonging to this species was taken by the Michael Sars in 1365 meters (ca. $28^{\circ} \mathrm{N}$., $13^{\circ} \mathrm{W}$.). Indo-Pacific species of the family are considered by some authors to be forms of $H$. raleighana. None of these has been reported from deep-abyssal waters.

\section{TELEOSTOMI}

\section{Family ALEPOCEPHALIDAE}

Although certain species of the families Alepocephalidae and Searsiidae ${ }^{1}$ are certainly pelagic, there is reason to believe that others may be benthic forms, having been caught principally or exclusively in apparatus fishing on bottom. The possibility that some species live pelagically as young needs investigation. All but two of the alepocephalids reported from depths greater than 3660 meters were caught in pelagic nets, and the two exceptions are known from only one specimen each. In deep-abyssal waters there are about thirty-two more species that may have been caught on or just above the ocean floor, most of them represented by too few specimens for evaluation and a few showing a preference for shallower depths. Species of the families Alepocephalidae and Searsiidae taken in pelagic nets hauled below 2000 meters and known from only one or two specimens have been included here in case the species should eventually prove to be benthic or partially benthic in habitat. Many of these fishes probably belong to the benthopelagic fauna found just above the ocean floor.

${ }^{1}$ Separated from the Alepocephalidae by Parr (1951). 
It has been impossible to determine the vertical distribution of the comparatively common, widely distributed, pelagic species Searsia koefoedi Parr (family Searsiidae), of which there are at least sixteen catches recorded in nets hauled below a depth of 2000 meters, among many more above that depth. Most of the specimens are apparently young, but a survey of the literature reveals no relation between size and depth of capture.

\section{Alepocephalus rostratus Risso}

Alepocephalus rostratus Risso, *1820, Mem. Accad. Sci. Torino, 25: 291, pl. 10, fig. 4-Mediterranean.

Alepocephalus rostratus Risso, 1826, Hist. Nat. Eur. Merid., 3: 449, pl. 11, fig. 28; Sassi, *1846, Descr. Genova Genov., Pesci, 1: 111; Cuvier and Valenciennes, 1846, Hist. Nat. Poiss., 19: 169, pl. 566; Canestrini, *1861, Arch. Zool. Anat. Fisiol. Genova, 2: 262; Johnson, 1862, Ann. Mag. Nat. Hist., (3), 10 : 285; Günther, 1868, Cat. Fishes Brit. Mus., 7 : 477; 1887, Rep. Sci. Res. Voy. Challenger, Zool., 22: 223; Gegenbaur, *1878, Morph. Jahrb., 4, Suppl., p. 1, pls. 1, 2; Moreau, 1881, Hist. Nat. Poiss. France, 3: 463, fig. 198; Vaillant, 1888, Exp. Sci. Trav. Talis., Poiss., p. 148, pl. 11, fig. 1, and pl. 12, fig. 5; Carus, 1893, Prodr. Faunae Medit., 2: 550; Goode and Bean, 1895, Ocean. Ichth., p. 36, fig. 41; Parona, *1898, Atti Soc. Ligure Sci. Nat. Geogr., 9: 327; Griffini, *1903, Ittiol. ital., Milano; Ariola, *1904, Aquivolt. Lomb., Milano, 5; Holt and Byrne, 1906, Rep. Fish. Ireland 1905, Sci. Invest., 2: 25; 1908, op. cit., 1906, 9: 32, pl. 3, fig. 1; Roule, 1919, Rés. Camp. Sci. Monaco, 52: 5; Koefoed, 1927, Rep. Sci. Res. M. Sars No. Atl. Deep-sea Exp. 1910, 4, (1), p. 32, pl. 1, fig. 2; Gregory, 1933, Trans. Amer. Phil. Soc., 23, (2), p. 158, fig. 51; ?Roule and Angel, 1933, Rés. Camp. Sci. Monaco, 86:4; Noronha and Sarmento, *1934, Peixes Madeira, pp. 105, 134; Fowler, 1936, Bull. Amer. Mus. Nat. Hist., 70: 185, fig. 74; Lozano Rey, 1947, Mem. R. Acad. Cien. Madrid, Ser. Cien. Nat., 11: 67, fig. 8; Maul, 1948, Bol. Mus. Mun. Funchal, 3: 10, fig. 2; Tortonese and Trotti, 1949, Atti Accad. Ligure Sci. Lett., 6, (1), p. 34; Arté, 1951, Publ. Inst. Biol. Aplic. Barcelona, 8: 157, fig. 1; Parr, 1952, Bull. Mus. Comp. Zool., 107: 257.

?Alepocephalus sp. Fage, 1910, Ann. Inst. Océanogr. Paris, 1, (7), p. 4, fig. 2.

Deep-abyssal records.-Eastern Atlantic, off Cape Verde, 3655 meters, one specimen.

Eastern Atlantic, off North Africa, 2330, 2190, 2115 meters, five specimens.

Eastern Atlantic, off the Azores, 2235 meters, one specimen.

Distribution.-Eastern Atlantic, $16^{\circ}-51^{\circ} \mathrm{N} ., 3^{\circ}-28^{\circ} \mathrm{W}$., at least eighty-three specimens recorded, seven from deep-abyssal waters and the rest in 300-1804 meters. Mediterranean at Nice and near Blanes, Spain, two specimens. (?) Western Atlantic, ca. $42^{\circ} \mathrm{N}$., 
$62^{\circ}-63^{\circ} \mathrm{W}$., three specimens, $1332-1458$ meters. Center of distribution probably between 1000 and 2000 meters.

Length. $-70-698 \mathrm{~mm}$.

Remarks.-The western Atlantic record was based on three damaged specimens (Roule and Angel, 1933, p. 4), which should be re-examined before being assigned to this species.

\section{Alepocephalus agassizi Goode and Bean}

Alepocephalus agassizii Goode and Bean, 1883, Bull. Mus. Comp. Zool., 10 : 218 -Atlantic off U. S. coast, ca. $38^{\circ}$ N., $73^{\circ}$ W., 1687 meters.

Alepocephalus agassizii Günther, 1887, Rep. Sci. Res. Voy. Challenger, Zool., 22: 223; Goode and Bean, 1895, Ocean. Ichth., p. 37, fig. 45; Jordan and Evermann, 1896, Bull. U. S. Nat. Mus., 47: 453; 1900, op. cit., fig. 197; Lütken, 1898, Danish Ingolf Exp., 2, (1), p. 8; Ehrenbaum, 1902, Fauna Arct., 2: 139; Koefoed, 1927, Rep. Sci. Res. M. Sars No. Atl. Deep-sea Exp. 1910, 4, (1), p. 36; Schroeder, 1940, Copeia, p. 231; Jensen, 1948, Skr. Univ. Zool. Mus. København, 9: 64; Parr, 1952, Bull. Mus. Comp. Zool., 107 : 258.

Deep-abyssal records.-Western Atlantic, $39^{\circ}-40^{\circ} \mathrm{N}$., $68^{\circ}-69^{\circ} \mathrm{W}$, 2012-2286 meters, at least four specimens, from four hauls.

Eastern Atlantic, ca. $57^{\circ} \mathrm{N} ., 11^{\circ}$ W., 1853 meters, three specimens.

Distribution.-North Atlantic from Davis Strait (ca. $63^{\circ}$ N.) and southwest of Iceland (ca. $62^{\circ} \mathrm{N}$., $28^{\circ} \mathrm{W}$.) to off Ireland in the east (ca. $50^{\circ} \mathrm{N} ., 11^{\circ} \mathrm{W}$.) and to $36^{\circ} \mathrm{N}$. in the west, with one capture in ca. $15^{\circ} \mathrm{N}$., $63^{\circ} \mathrm{W}$. At least twenty-four specimens known, seven of them deep-abyssal, others in 984-1797 meters. Perhaps not rare in deep-abyssal waters, although the center of distribution may be higher.

Length. $-222-642 \mathrm{~mm}$.

\section{Alepocephalus productus Gill}

Alepocephalus productus Gill, 1883, Proc. U. S. Nat. Mus., 6: 256-Atlantic off U. S. coast, ca. $39^{\circ}$ N., $70^{\circ}$ W., 2491 meters.

Alepocephalus productus Günther, 1887, Rep. Sci. Res. Voy. Challenger, Zool., 22: 223; Goode and Bean, 1895, Ocean. Ichth., p. 37, fig. 46; Jordan and Evermann, 1896, Bull. U. S. Nat. Mus., 47: 452; Koefoed, 1927, Rep. Sci. Res. M. Sars No. Atl. Deep-sea Exp. 1910, 4, (1), p. 38; Parr, 1952, Bull. Mus. Comp. Zool., 107: 258.

Deep-abyssal records and distribution.-Western Atlantic, 2491 meters, the type. 
Western Atlantic, Gulf of Mexico, 2104-2194 meters, one specimen (Oregon Station 1303, $28^{\circ} 47^{\prime}$ N., $87^{\circ} 50^{\prime}$ W., May 26, 1955).

Eastern Atlantic, ca. $35^{\circ}$ N., $8^{\circ}$ W., 2055 meters, one specimen.

Length. $-345-360 \mathrm{~mm}$. without caudal. Length of type not stated.

\section{Alepocephalus fundulus Garman}

Alepocephalus fundulus Garman, 1899, Mem. Mus. Comp. Zool., 24: 293, pl. 57, fig. 2-Pacific off Panama, $6^{\circ}-7^{\circ}$ N., $79^{\circ}-82^{\circ}$ W. (type locality not designated).

Alepocephalus fundulus Parr, 1952, Bull. Mus. Comp. Zool., 107: 258.

Deep-abyssal records and distribution.-Eastern Pacific, 3057, 2322 meters, two records.

Length.-?-419 $\mathrm{mm}$.

\section{Alepocephalus australis Barnard}

Alepocephalus australis Barnard, 1923, Ann. So. Afr. Mus., 13: 440-off Cape Point, South Africa, 1153 meters.

Alepocephalus australis Barnard, 1925, Ann. So. Afr. Mus., 21 : 121; ?Koefoed, 1927, Rep. Sci. Res. M. Sars No. Atl. Deep-sea Exp. 1910, 4, (1), p. 39, pl. 4, figs. 5, 6; Smith, 1949, Sea Fishes So. Afr., p. 96, fig. 124; Parr, 1952, Bull. Mus. Comp. Zool., 107: 257.

Bathytroctes rostratus Barnard, 1925, Ann. So. Afr. Mus., 21: 122.

Alepocephalus barnardi Norman, 1930, Disc. Rep., 2: 270; Smith, 1949, Sea Fishes So. Afr., p. 96.

Alepocephalus australis barnardi Parr, 1952, Bull. Mus. Comp. Zool., 107: 257.

Deep-abyssal record.-Eastern Atlantic, ca. $27^{\circ}$ N., $14^{\circ}$ W., 2603 meters, two specimens, somewhat doubtfully assigned to this species.

Distribution.- South Atlantic off Cape Point, five specimens in 1153 and one in 1280 meters. Eastern Atlantic, one deep-abyssal record and one specimen, doubtfully identified with this species, in 1797 meters (ca. $50^{\circ} \mathrm{N} ., 11^{\circ} \mathrm{W}$.).

Length.-200-500 mm.

\section{Alepocephalus atlanticus Roule and Angel}

Alepocephalus edentulus atlanticus Roule and Angel, 1931, Bull. Inst. Océanogr. Monaco, 581: 5-Atlantic, ca. $32^{\circ} \mathrm{N} ., 12^{\circ} \mathrm{W}$.

Alepocephalus edcntulus atlanticus Roule and Angel, 1933, Rés. Camp. Sci. Monaco, 86: 4, pl. 1, fig. 1; Belloc, 1949, Bull. Inst. Océanogr. Monaco, 958: 5 .

Alepocephalus atlanticus Fowler, 1936, Bull. Amer. Mus. Nat. Hist., 70: 1161. 
Deep-abyssal record and distribution.-Eastern Atlantic, 4000 meters, one specimen taken in a pelagic net.

Length.--195 mm.

\section{Talismania bifurcata Parr}

Nemabathytroctes bifurcatus Parr, 1951, Amer. Mus. Nov., 1531: 10-Pacific off Panama, ca. $7^{\circ} \mathrm{N}$., $79^{\circ} \mathrm{W}$.

Talismania bifurcata Parr, 1952, Jour. Washington Acad. Sci., 42: 269.

Deep-abyssal record and distribution.-Eastern Pacific, ?2200 meters, one specimen taken in a pelagic net with 3500 meters of wire out. Depth to bottom 2550 meters. Probably caught on or near bottom.

Length. $-202 \mathrm{~mm}$.

\section{Rinoctes nasutus Koefoed}

Bathytroctes nasutus Koefoed, 1927, Rep. Sci. Res. M. Sars No. Atl. Deep-sea Exp. 1910,4 , (1), p. 50, pl. 3, fig. 10 -Atlantic, ca. $34^{\circ}$ N., $33^{\circ}$ W., 2865 meters.

Rinoctes nasutus Parr, 1952, Bull. Mus. Comp. Zool., 107: 264.

Deep-abyssal records and distribution.-Western Atlantic, ca. $20^{\circ}$ N., $80^{\circ}$ W., 3886 meters, one specimen.

Eastern Atlantic, 2865 meters, the type.

Length.-122 (standard) $-190 \mathrm{~mm}$.

\section{Bathytroctes alveatus Garman}

Bathytroctes alveatus Garman, 1899, Mem. Mus. Comp. Zool., 24: 287, pl. 58, fig. 1-Pacific off Colombia and Ecuador, ca. $3^{\circ} \mathrm{N}$., $82^{\circ} \mathrm{W}$. and $0^{\circ} 36^{\prime} \mathrm{S}$., $86^{\circ} \mathrm{W}$. (type locality not designated).

Deep-abyssal records and distribution.-Eastern Pacific, 2415, 2068 meters, an unstated number of specimens from two hauls.

Length.--?-190.5 mm.

\section{Bathy troctes macrolepis Günther}

Bathytroctes macrolepis Günther, 1887, Rep. Sci. Res. Voy. Challenger, Zool., 22: 225 , pl. 57 , fig. B-Pacific north of Celebes, ca. $2^{\circ} \mathrm{N} ., 124^{\circ} \mathrm{E}$.

Bathytroctes macrolepis Goode and Bean, 1895, Ocean. Ichth., p. 41, fig. 44;

(?) Alcock, 1899, Descr. Cat. Indian Deep-sea Fishes, p. 174; Weber and

de Beaufort, 1913, Fishes Indo-Austr. Arch., 2: 103; (?) Misra, 1950, Rec.

Indian Mus., 45: 408; (?) 1953, op. cit., 50: 390; Parr, 1952, Bull. Mus.

Comp. Zool., 107: 267.

Deep-abyssal record.-Western Pacific, 3932 meters, the type. 
Distribution.-Western Pacific, one deep-abyssal record. (?) Indian Ocean, Andaman Sea, one damaged specimen, depth of capture not known.

Length. $-229 \mathrm{~mm}$.

\section{Bathytroctes inspector Garman}

Bathytroctes inspector Garman, 1899, Mem. Mus. Comp. Zool., 24: 288, pl. M, fig. 1-Pacific off Panama, ca. $6^{\circ}$ N., $83^{\circ} \mathrm{W}$.

Bathytroctes inspector Parr, 1952, Bull. Mus. Comp. Zool., 107: 266.

Deep-abyssal record and distribution.-Eastern Pacific, 2690 meters, one(?) specimen.

Length. $-254 \mathrm{~mm}$.

\section{Bathytroctes alvifrons Garman}

Bathytroctes alvifrons Garman, 1899, Mem. Mus. Comp. Zool., 24: 286, pl. 58, fig. 2-Pacific off Panama and the Galapagos Islands, ca. $6^{\circ} \mathrm{N} ., 80^{\circ} \mathrm{W}$. and ca. $2^{\circ} \mathrm{N} ., 92^{\circ} \mathrm{W}$. (type locality not designated).

Bathytroctes alvifrons Parr, 1952, Bull. Mus. Comp. Zool., 107: 267.

Deep-abyssal records and distribution.-Eastern Pacific, 3279, 2488 meters, an unstated number of specimens from two hauls.

Length.-?--ca. $229 \mathrm{~mm}$.

\section{Bathytroctes koefoedi Parr}

Bathytroctes koefoedi Parr, 1951, Amer. Mus. Nov., 1531: 11-Atlantic, ca. $24^{\circ}$ N., $33^{\circ} \mathrm{W}$.

Bathytroctes alvifrons Koefoed, 1927, Rep. Sci. Res. M. Sars No. Atl. Deep-sea Exp. 1910, 4, (1), p. 45.

Bathytroctes koefoedi Parr, 1952, Bull. Mus. Comp. Zool., 107: 267.

Deep-abyssal record and distribution.-Eastern Atlantic, 2865 meters, nineteen specimens in one haul.

Length.-105-400 mm.

\section{Bathytroctes michaelsarsi Koefoed}

Bathytroctes michaelsarsi Koefoed, 1927, Rep. Sci. Res. M. Sars No. Atl. Deep-sea Exp. 1910, 4, (1), p. 48, pl. 3, fig. 9 -Atlantic, ca. $34^{\circ}$ N., $33^{\circ} \mathrm{W}$.

Bathytroctes michaelsarsi Parr, 1952, Bull. Mus. Comp. Zool., 107: 266.

Deep-abyssal record and distribution.--Eastern Atlantic, 2865 meters, four specimens in one haul.

Length. $-260-420 \mathrm{~mm}$. 


\section{Bajacalifornia drakei Beebe}

Bathytroctes drakei Beebe, 1929, Zoologica, 12:6-off New York, 1463 meters.

Bathytroctes drakei Beebe, 1933, op. cit., 16: 10, 23, figs. 2-5; 1937, op. cit., 22: 198 .

Bajacalifornia drakei Parr, 1937, Bull. Bingham Oceanogr. Coll., 3, (7), pp. 11, 24, figs. 1, 8; 1952, Bull. Mus. Comp. Zool., $107: 265$.

Deep-abyssal record.-Western Atlantic off the Bahamas, 2012 meters, one specimen doubtfully assigned to this species.

Distribution.-Western Atlantic off New York, Bermuda, and the Bahamas, one deep-abyssal record and twenty-seven juvenile specimens in $732-1645$ meters.

Length. $-10-68 \mathrm{~mm}$. (without caudal).

\section{Grimatroctes grimaldii Zugmayer}

Bathytroctes Grimaldii Zugmayer, 1911, Bull. Inst. Océanogr. Monaco, 193: 1-Atlantic, ca. $37^{\circ} \mathrm{N} ., 10^{\circ} \mathrm{W}$.

Bathytroctes Grimaldii Zugmayer, 1911, Rés. Camp. Sci. Monaco, 35: 6, pl. 1, fig. 2; Lozano Rey, 1947, Mem. R. Acad. Cien. Madrid, Ser. Cien. Nat,, 11 : 73, pl. 3, fig. 2; Belloc, 1949, Bull. Inst. Océanogr. Monaco, 958: 5.

Grimatroctes grimaldi Parr, 1952, Bull. Mus. Comp. Zool., 107: 266.

Deep-abyssal record and distribution.--Eastern Atlantic, 4900 meters, one specimen caught in a pelagic net.

Length. $-165 \mathrm{~mm}$.

\section{Grimatroctes microlepis Günther}

Bathytroctes microlepis Günther, 1878, Ann. Mag. Nat. Hist., (5), 2: 249 Atlantic off Portugal, ca. $35^{\circ} \mathrm{N} ., 8^{\circ} \mathrm{W}$.

Bathytroctes microlepis Günther, 1887, Rep. Sci. Res. Voy. Challenger, Zool., 22 : 226, pl. 57, fig. A; Goode and Bean, 1895, Ocean. Ichth., p. 42; Roule, 1935, Bull. Inst. Océanogr. Monaco, 674: 4; Nobre, 1935, Fauna Mar. Portugal, 1: 519; Lozano Rey, 1947, Mem. R. Acad. Cien. Madrid, Ser. Cien. Nat., $11: 76$, fig. 11.

Grimatroctes microlepis Parr, 1952, Bull. Mus. Comp. Zool., 107 : 266.

Deep-abyssal record and distribution.-Eastern Atlantic, 1992 meters, one specimen.

Length. $-254 \mathrm{~mm}$.

Remarks.-Roule (1935, p. 4) reported a somewhat damaged specimen taken from the stomach of a tuna caught at Madeira, and Alcock (1899, p. 174) doubtfully identified as this species a mutilated fish from a depth of 914 meters in the Andaman Sea. 


\section{Narcetes pappenheimi Fowler}

Narcetes pappenheimi Fowler, 1934, Proc. Acad. Nat. Sci. Philadelphia, 85: 253, fig. 15-Gulf of Tomini, Celebes.

Deep-abyssal record and distribution.-Western Pacific, 1997 meters, one specimen.

Length. $-128 \mathrm{~mm}$.

\section{Narcetes stomias Gilbert}

Bathytroctes stomias Gilbert, 1890, Proc. U. S. Nat. Mus., 13: 53-Pacific off Oregon, ca. $47^{\circ} \mathrm{N}$., $125^{\circ} \mathrm{W} ., 1604$ meters.

Bathytroctes stomias Goode and Bean, 1895, Ocean. Ichth., p. 40; Jordan and Evermann, 1896, Bull. U. S. Nat. Mus., $47: 454$.

Narcetes stomias Townsend and Nichols, 1925, Bull. Amer. Mus. Nat. Hist., 52: 10 .

Deep-abyssal record.-Eastern Pacific off San Diego, 1968 meters, one specimen.

Distribution.-Eastern Pacific off San Diego and Oregon, one deep-abyssal specimen and one in 1604 meters.

Length. $-330-381 \mathrm{~mm}$.

Remarks.-An examination of two specimens of Narcetes captured recently at a depth of 2104-2194 meters in the Gulf of Mexico suggests that $N$. affinis, $N$. pluriserialis, and the two eastern Atlantic specimens recorded by Koefoed (1927, p. 54) under the name pluriserialis are probably synonyms of $N$. stomias. The new specimens will be described in detail later. Koefoed's examples were taken in ca. $35^{\circ} \mathrm{N} ., 8^{\circ} \mathrm{W}$., in 2055 meters and ca. $50^{\circ} \mathrm{N} ., 11^{\circ} \mathrm{W}$, in 1797 meters.

\section{Narcetes pluriserialis Garman}

Narcetes pluriserialis Garman, 1899, Mem. Mus. Comp. Zool., 24: 289, pl. 57, fig. 3-Pacific off Panama, ca. $5^{\circ}$ N., $86^{\circ}$ W., 1847 meters.

Deep-abyssal record and distribution.-Eastern Pacific off Panama, 1847 meters, an unstated number of specimens from one haul.

Length.-Ca. $432 \mathrm{~mm}$.

\section{Narcetes affinis Lloyd}

Narcetes affinis Lloyd, 1906, Ann. Mag. Nat. Hist., (7), 18: 308-Gulf of Oman, Indian Ocean.

Narcetes affinis Annandale and Lloyd, 1908, Ill. Zool. Investigator, Fishes, pl. 42, figs. 1, 1, $a$; Lloyd, 1909, Mem. Indian Mus., 2: 149; Misra, 1950, Rec. Indian Mus., 45: 408; 1953, op. cit., 50: 391. 
Deep-abyssal record and distribution.-North Indian Ocean, 1839 meters, one specimen.

Length. $-356 \mathrm{~mm}$.

\section{Bellocia vaillanti Parr}

Bellocia vaillanti Parr, 1951, Amer. Mus. Nov., 1531: 6, 12-off Morocco, Talisman Station $28 .{ }^{1}$

Bathytroctes melanocephalus Vaillant, 1888, Exp. Sci. Trav. Talis., Poiss., pl. 11, fig. 3 (not description on p. 155); Bertin, 1940, Bull. Mus. Hist. Nat. Paris, 12: 274 (part).

Deep-abyssal record and distribution.-Eastern Atlantic, 2600 meters, one specimen (see discussion on p. 118).

Length.-104.5 mm. (without caudal).

\section{Leptochilichthys macrops Roule and Angel}

Leptochilichthys Agassizii macrops Roule and Angel, 1931, Bull. Inst. Océanogr. Monaco, 581 : 6-Atlantic off Morocco, ca. $34^{\circ}$ N., $10^{\circ} \mathrm{W}$.

Leptochilichthys Agassizii macrops Roule and Angel, 1933, Rés. Camp. Sci. Monaco, 86: 8, pl. 1, fig. 3; Belloc, 1949, Bull. Inst. Océanogr. Monaco, 958: 5 .

Leptochilichthys macrops Fowler, 1936, Bull. Amer. Mus. Nat. Hist., 70: 1162, fig. 490.

Deep-abyssal record and distribution.-Eastern Atlantic, 3000 meters, one specimen taken in a pelagic net.

Length. $-275 \mathrm{~mm}$.

Remarks. - Closely related to the following species.

\section{Leptochilichthys agassizi Garman}

Leptochilichthys Agassizii Garman, 1899, Mem. Mus. Comp. Zool., 24: 285, pl. 58, fig. 3 -Pacific off Ecuador, ca. $1^{\circ}$ N., $80^{\circ} \mathrm{W}$.

Deep-abyssal record and distribution.--Eastern Pacific, 2877 meters, one(?) specimen.

\section{Length. $-305 \mathrm{~mm}$.}

${ }^{1}$ Sanderson Smith (1888, p. 980) published a list of the dredgings made by the French ships Travailleur in 1880-81 and Talisman in 1883. It has been found that the data therein rarely agree with that given by Vaillant (1888). For instance, Bertin (1940, p. 275) stated that the type of Bathytroctes attritus Vaillant was from Talisman Station 121. Both he and Vaillant cite this locality as south of the Azores in 1442 meters, but Smith's data for the station are as follows: $16^{\circ} 52^{\prime}$ N., $25^{\circ} 12^{\prime}$ W., depth 508 meters, Cape Verde Islands. For the same species Vaillant lists Station 100, Banc d'Árguin, 1550 meters, while Smith lists the depth of Station 100 as 2324 meters. Similar contradictions are found more or less consistently. In view of these discrepancies, and since the original Talisman station records are not at hand, exact type localities cannot be given for Vaillant's fishes. 


\section{Rouleina attritus Vaillant}

Bathytroctes attritus Vaillant, 1888, Exp. Sci. Trav. Talis., Poiss., p. 158, pl. 12, fig. 2-Atlantic south of the Azores, Talisman Station 121, 1442 meters (see footnote, p. 110).

Bathytroctes attritus Goode and Bean, 1895, Ocean. Ichth., p. 45; Vaillant, in Roule, 1919, Rés. Camp. Sci. Monaco, 52: 129; Bertin, 1940, Bull. Mus. Hist. Nat. Paris, 12: 275.

Bathytroctes mollis Koehler, 1896, Ann. Univ. Lyon, 26: 517, pl. 26, fig. 2; Fowler, 1936, Bull. Amer. Mus. Nat. Hist., 70: 189, fig. 77; Lozano Rey, 1947, Mem. R. Acad. Cien. Madrid, Ser. Cien. Nat., 11: 80, fig. 13.

Talismania mollis Roule, 1916, Bull. Inst. Océanogr. Monaco, 320: 11; 1919, Rés. Camp. Sci. Monaco, 52: 6, pl. 1, fig. 1; 1927, Bull. Inst. Océanogr. Monaco, 497: 2; 1934, Poiss. Monde Viv. Eaux, 7: 151, pl. 6, text fig.; Koefoed, 1927, Rep. Sci. Res. M. Sars No. Atl. Deep-sea Exp. 1910, 4, (1), p. 55, pl. 3 , fig. 5 .

Caudania mollis Roule, 1935, Bull. Inst. Océanogr. Monaco, 674: 2; Nybelin, 1948, Göteborgs K. Vetensk. Vitt.-Samh. Handl., (B), 5, (16), p. 9, pl. 1, fig. 2 , text fig. 3 .

Alepocephalus rostratus Fowler, 1936, Bull. Amer. Mus. Nat. Hist., 70: 185 (part).

Rouleina attritus Parr, 1951, Amer. Mus. Nov., 1531 : 13.

Deep-abyssal records.-Eastern Atlantic off Cape Verde, 3655 meters, one specimen.

Eastern Atlantic, ca. $35^{\circ}$ N., $8^{\circ}$ W., 2055 meters, eleven specimens.

Eastern Atlantic, ca. $35^{\circ}$ N., $8^{\circ}$ W., 2150-2300 meters, one specimen.

Distribution.-Eastern Atlantic only, $16^{\circ}-38^{\circ}$ N., $4^{\circ}-26^{\circ} \mathrm{W}$, twenty-six specimens, thirteen in deep-abyssal waters and thirteen in 1442-1805 meters. Said to be not rare at Madeira, depth unknown (Roule, 1927, p. 2).

Length. $-130-460 \mathrm{~mm}$.

\section{Rouleina danae Parr}

Rouleina danae Parr, 1951, Amer. Mus. Nov., 1531: 14-Pacific, ca. $8^{\circ}$ N., $119^{\circ} \mathrm{E}$.

Deep-abyssal record and distribution.-Western Pacific, Sulu Sea, ?2500 meters, one specimen, 4000 meters of wire out, depth to bottom 2775 meters. Probably caught on or near bottom.

Length. $-108 \mathrm{~mm}$. (without caudal).

\section{Rouleina guentheri Alcock}

Xenodermichthys Guentheri Alcock, 1892, Ann. Mag. Nat. Hist., (6), 10: 359, pl. 18, fig. 3-Bay of Bengal, 1240 meters. 
Xenodermichthys guntheri (guentheri of authors) Alcock, 1899, Descr. Cat. Indian Deep-sea Fishes, p. 180; Alcock and McArdle, 1900, Ill. Zool. Investigator, Fishes, pl. 32, fig. 2; McArdle, 1901, Ann. Mag. Nat. Hist., (7), 8: 518; Misra, 1950, Rec. Indian Mus., 45: 409; 1953, op. cit., 50: 391 , fig. $12, b$.

Rouleina guntheri Norman, 1930, Disc. Rep., 2: 271; 1939, Sci. Rep. John Murray Exp. 1933-34, 7, (1), p. 15; Parr, 1951, Amer. Mus. Nov., 1531: 18.

Deep-abyssal records.-Indian Ocean, ca. $10^{\circ}$ N., $74^{\circ}$ E., 21042140 meters, (?)specimens.

Indian Ocean, ca. $7^{\circ} \mathrm{N} ., 76^{\circ}$ E., 1840 meters, (?)specimens.

Distribution.-Indian Ocean, Bay of Bengal, Arabian Sea and Gulf of Aden, two deep-abyssal records and at least six specimens in $786-1410$ meters.

Length. $-110-152 \mathrm{~mm}$.

\section{Asquamiceps velaris Zugmayer}

Asquamiceps velaris Zugmayer, 1911, Bull. Inst. Océanogr. Monaco, 193: 2 -Atlantic, ca. $36^{\circ}$ N., $9^{\circ} \mathrm{W}$.

Asquamiceps velaris Zugmayer, 1911, Rés. Camp. Sci. Monaco, 35: 10, pl. 1, fig. 4; Fowler, 1936, Bull. Amer. Mus. Nat. Hist., 70: 186, fig. 75; Lozano Rey, 1947, Mem. R. Acad. Cien. Madrid, Ser. Cien. Nat., 11: 82, pl. 3, fig. 4; Belloc, 1949, Bull. Inst. Océanogr. Monaco, 958: 5.

Asquamiceps (Asquamiceps) velaris Parr, 1954, Amer. Mus. Nov., 1655: 4.

Deep-abyssal record and distribution.-Eastern Atlantic, 3660 meters, one specimen, in a pelagic net.

Length. $-175 \mathrm{~mm}$.

\section{Asquamiceps indagatio Parr}

Asquamiceps (Asquamiceps) indagatio Parr, 1954, Amer. Mus. Nov., 1655: 4, 5-Atlantic off South Africa, ca. $34^{\circ} \mathrm{S} ., 16^{\circ} \mathrm{E}$.

Asquamiceps relaris Norman, 1930, Disc. Rep., 2: 267.

Deep-abyssal record and distribution.-Atlantic off South Africa, 2580 meters, one specimen taken in a closing net.

Length. $-90 \mathrm{~mm}$. (without caudal).

\section{Asquamiceps pacificus Parr}

Asquamiceps (Perioceps) pacificus Parr, 1954, Amer. Mus. Nov., 1655: 4, 6 -Gulf of Panama, ca. $6^{\circ} \mathrm{N} ., 80^{\circ} \mathrm{W}$.

Deep-abyssal record and distribution.-Eastern Pacific, 2200 meters, one specimen taken in a pelagic net, with 3500 meters of wire out. Depth to bottom not known.

Length. $-85 \mathrm{~mm}$. (without caudal). 


\section{Brunichthys asperifrons Garman}

Alepocephalus asperifrons Garman, 1899, Mem. Mus. Comp. Zool., 24: 291, pl. 59, fig. $1-$ Pacific off Panama, $6^{\circ}-7^{\circ}$ N., $79^{\circ}-81^{\circ}$ W. (type locality not designated).

Brunichthys asperifrons Parr, 1951, Amer. Mus. Nov., 1531: 8.

Deep-abyssal record.-Eastern Pacific, 1865 meters, one haul.

Distribution.-Eastern Pacific, one deep-abyssal record and one haul in 1430 meters.

Length.--?-305 mm.

\section{Leptoderma macrops Vaillant}

Leptoderma macrops Vaillant, 1888, Exp. Sci. Trav. Talis., Poiss., pp. 31, 166, pl. 13, fig. 2-Atlantic between the Canary Islands and Morocco, Talisman Station 47 (see footnote, p. 110).

Leptoderma macrops Goode and Bean, 1895, Ocean. Ichth., p. 49, fig. 56; Koehler, 1896, Ann. Univ. Lyon, 26: 523; Roule and Angel, 1933, Rés. Camp. Sci. Monaco, 86: 8; Fowler, 1936, Bull. Amer. Mus. Nat. Hist., 70: 193, fig. 82; Bertin, 1940, Bull. Mus. Hist. Nat. Paris, 12: 275; Lozano Rey, 1947, Mem. R. Acad. Cien. Madrid, Ser. Cien. Nat., 11: 92, fig. 17; Tucker, 1954, Bull. Brit. Mus. (Nat. Hist.), Zool., 2: 183, figs. 9-13.

Deep-abyssal record.-Eastern Atlantic off North Africa, 2330 meters, one specimen.

Distribution.-Eastern Atlantic only, off North Africa, the Canary Islands and in the Gulf of Gascony, at least sixty-three specimens, one from deep-abyssal waters and the rest in 800-1804 meters. Center of distribution probably between 1000 and 2000 meters.

Length. -85 (standard) $-195 \mathrm{~mm}$.

\section{Aulastomatomorpha phosphorops Alcock}

Aulastomatomorpha phosphorops Alcock, 1890, Ann. Mag. Nat. Hist., (6), 6: 307 -Indian Ocean, ca. $11^{\circ}$ N., $74^{\circ}$ E., 1829 meters.

A ulastomatomorpha phosphorops Alcock, 1891, Ann. Mag. Nat. Hist., (6), 7 : 10, fig. 1; 1892, Ill. Zool. Investigator, Fishes, pl. 5, fig. 2; Goode and Bean, 1895, Ocean. Ichth., p. 50, fig. 55; Lloyd, 1906, Ann. Mag. Nat. Hist., (7), 18: 306, fig.; 1909, Mem. Indian Mus., 2: 148; Misra, 1950, Rec. Indian Mus., 45: 410; 1953, op. cit., 50: 393, fig. 14, b.

Aulastomomorpha phosphorops Alcock, 1899, Descr. Cat. Indian Deep-sea Fishes, p. 178; 1902, Nat. Indian Seas, p. 239, fig. 40.

Deep-abyssal records and distribution.-Bay of Bengal, 2012 meters, one specimen.

Arabian Sea, 1829 meters, one specimen.

Length. - $279 \mathrm{~mm}$. 


\section{Aulastomatomorpha caeruliceps Lloyd}

Aulastomatomorpha caeruliceps Lloyd, 1906, Ann. Mag. Nat. Hist., (7), 18: 308-Gulf of Oman.

Aulastomomorpha caeruliceps Annandale and Lloyd, 1908, Ill. Zool. Investigator, Fishes, pl. 42, fig. 3 .

Aulastomatomorpha caeruliceps Lloyd, 1909, Mem. Indian Mus., 2: 148.

Deep-abyssal record and distribution.-Arabian Sea, 1839 meters, one specimen.

Length. $-180 \mathrm{~mm}$.

\section{Conocara werneri Nybelin}

Conocara werneri Nybelin, 1946, Ark. Zool., 38B, (2), p. 1, fig. 1-Atlantic, ca. $35^{\circ} \mathrm{N} ., 8^{\circ} \mathrm{W}$.

Conocara werneri Nybelin, 1948, Göteborgs K. Vetensk. Vitt.-Samh. Handl., (B), 5, (16), p. 5, pl. 1, fig. 1.

Deep-abyssal record and distribution.-Eastern Atlantic, 21502300 meters, one specimen.

Length. $-380 \mathrm{~mm}$.

\section{Conocara macroptera Vaillant}

Alepocephalus macropterus Vaillant, 1888, Exp. Sci. Trav. Talis., Poiss., p. 150, pl. 11, fig. 2-North Africa, Banc d'Arguin, 1550 meters, Talisman Station 100 (see footnote, p. 110).

Conocara macroptera Goode and Bean, 1895, Ocean. Ichth., p. 39, fig. 43; Jordan and Evermann, 1896, Bull. U.S. Nat. Mus., 47: 458; Murray and Hjort, 1912, Depths of Ocean, p. 394, fig. 263; Roule, 1919, Rés. Camp. Sci. Monaco, 52: 5; Roule and Angel, 1933, op. cit., 86: 5; Fowler, 1936, Bull. Amer. Mus. Nat. Hist., 70: 190, 1163, fig. 79; ?Parr, 1937, Bull. Bingham Oceanogr. Coll., 3, (7), p. 10, fig. 1; Lozano Rey, 1947, Mem. R. Acad. Cien. Madrid, Ser. Cien. Nat., 11: 90, pl. 4, fig. 1; Nybelin, 1948, Göteborgs K. Vetensk. Vitt.-Samh. Handl., (B), 5, (16), p. 8.

Alepocephalus macropterus Holt and Byrne, 1906, Rep. Fish. Ireland 1905, Sci. Invest., 2: 25; 1908, Rep. Fish. Ireland 1906, Sci. Invest., 5: 42, pl. 5, fig. 1; Koefoed, 1927, Rep. Sci. Res. M. Sars No. Atl. Deep-sea Exp. 1910, 4, (1), p. 40, pl. 1, fig. 1 .

Alepocephalus (Conocara) macroptera Bertin, 1940, Bull. Mus. Hist. Nat. Paris, 12: 273.

Deep-abyssal records.-Eastern Atlantic off Morocco, 2055-2115 meters, ten specimens from three hauls.

Distribution.-Eastern Atlantic, $28^{\circ}-51^{\circ}$ N., $7^{\circ}-28^{\circ}$ W., ten specimens from deep-abyssal waters and twenty-four in 865-1805 meters. Western Atlantic in ca. $16^{\circ} \mathrm{N}$., $63^{\circ} \mathrm{W}$. and off the Bahamas 
(a doubtful identification), two specimens, 1257-1729 meters. Reaching deep-abyssal waters but with the maximum occurrence probably in 1400-1800 meters.

Length. -69 (standard) and 160-330 $\mathrm{mm}$.

\section{Conocara murrayi Koefoed}

Alepocephalus murrayi Koefoed, 1927, Rep. Sci. Res. M. Sars No. Atl. Deep-sea Exp. 1910, 4, (1), p. 41, pl. 3, fig. 6, text figs. 9, 10 -Atlantic, ca. $35^{\circ} \mathrm{N}$, $8^{\circ} \mathrm{W} ., 2055$ meters.

Deep-abyssal records and distribution.-Eastern Atlantic, ca. $35^{\circ}$ N., $8^{\circ}$ W., 2603 meters, two specimens.

Eastern Atlantic, ca. $27^{\circ} \mathrm{N}$., $14^{\circ} \mathrm{W}$., 2055 meters, one specimen.

Western Atlantic, Gulf of Mexico, 2104-2194 meters, one specimen (Oregon Station 1303, $28^{\circ} 47^{\prime}$ N., $87^{\circ} 50^{\prime}$ W., May 26, 1955).

Length. $-157 \mathrm{~mm}$. (without caudal) and 230-290 $\mathrm{mm}$.

\section{Ericara salmonea Gill and Townsend}

Ericara salmonea Gill and Townsend, 1897, Proc. Biol. Soc. Washington, 11 : 232 -Bering Sea, ca. $55^{\circ}$ N., $170^{\circ} \mathrm{W}$.

Ericara salmonea Jordan and Evermann, 1898, Bull. U. S. Nat. Mus., 47 : 2816; Parr, 1951, Amer. Mus. Nov., 1531 : 15.

Xenognathus profundorum Gilbert, 1915, Proc. U. S. Nat. Mus., 48: 311, pl. 14.

Deep-abyssal records and distribution.-North Pacific, 3239 meters, the type.

Eastern Pacific off Catalina Island, 2469-3991 meters, one specimen.

Length.-? and $525 \mathrm{~mm}$.

\section{Ericara niger Günther}

Alepocephalus niger Günther, 1878, Ann. Mag. Nat. Hist., (5), 2 : 248 -Pacific north of Australia, ca. $12^{\circ} \mathrm{S} ., 145^{\circ} \mathrm{E}$.

Alepocephalus niger Günther, 1887, Rep. Sci. Res. Voy. Challenger, Zool., 22 : 224, pl. 56, fig. B; Goode and Bean, 1895, Ocean. Ichth., pp. 36, 38, fig. 42.

Whitleydea niger Parr, 1937, Bull. Bingham Oceanogr. Coll., 3, (7), pp. 6, 9,12 .

Ericara niger Parr, 1951, Amer. Mus. Nov., 1531 : 15.

Deep-abyssal record and distribution.-Western Pacific, 2560 meters, one specimen.

Length. $-330 \mathrm{~mm}$. 


\section{Einara macrolepis Koefoed}

Alepocephalus macrolepis Koefoed, 1927, Rep. Sci. Res. M. Sars No. Atl. Deep-sea Exp. 1910, 4, (1), p. 44, pl. 3, fig. 8, text figs. 12, 13-Atlantic, ca. $28^{\circ} \mathrm{N}, 2^{\circ} \mathrm{W}$.

Alepocephalus sp. Murray and Hjort, 1912, Depths of Ocean, p. 412.

Asquamiceps macrolepis Parr, 1937, Bull. Bingham Oceanogr. Coll., 3, (7), p. 12 .

Einara macrolepis Parr, 1951, Amer. Mus. Nov., 1531: 10.

Deep-abyssal record and distribution.-Eastern Atlantic, 4000 meters, one specimen caught in a pelagic net. Depth to bottom 5000 meters.

Length. $-200 \mathrm{~mm}$.

\section{Family SEARSIIDAE}

\section{Mirorictus taningi Parr}

Mirorictus taningi Parr, 1947, Copeia, p. 60, fig. 1-Gulf of Panama.

Deep-abyssal record and distribution.-Eastern Pacific, ?2200 meters, one specimen taken in a pelagic net, with 3500 meters of wire out. Depth to bottom not known.

Length. $-137 \mathrm{~mm}$.

\section{Platytroctes apus Günther}

Platytroctes apus Günther, 1878, Ann. Mag. Nat. Hist., (5), 2: 249-Atlantic, ca. $1^{\circ} \mathrm{N} ., 26^{\circ} \mathrm{W} ., 2744$ meters.

Platytroctes apus Günther, 1887, Rep. Sci. Res. Voy. Challenger, Zool., 22: 229, pl. 58, fig. A; Alcock, 1890, Ann. Mag. Nat. Hist., (6), 6: 307; 1891, op. cit., (6), 7: 11; 1899, Descr. Cat. Indian Deep-sea Fishes, p. 177; Goode and Bean, 1895, Ocean. Ichth., p. 46, fig. 53; Jordan and Evermann, 1896, Bull. U. S. Nat. Mus., 47: 458; Lloyd, 1909, Mem. Indian Mus., 2: 146; Zugmayer, 1911, Rés. Camp. Sci. Monaco, 35: 8; Roule, 1916, Bull. Inst. Océanogr. Monaco, 320 : 12; 1919, Rés. Camp. Sci. Monaco, 52 : 14, pl. 1, fig. 4; Koefoed, 1927, Rep. Sci. Res. M. Sars No. Atl. Deep-sea Exp. 1910, 4, (1), p. 58; Fowler, 1936, Bull. Amer. Mus. Nat. Hist., 70: 191, fig. 80 ; Lozano Rey, 1947, Mem. R. Acad. Cien. Madrid, Ser. Cien. Nat., 11 : 85, fig. 15; Misra, 1950, Rec. Indian Mus., 45: 409; 1953, op. cit., 50 : 391 .

?Platytroctes procerus Brauer, 1906, Wiss. Ergebn. Deutschen Tiefsee Exp. Valdivia, 15, (1), p. 23, fig. 3; 1908, op. cit., 15, (2), p. 192.

Deep-abyssal records.-Mid-Atlantic east of the mid-Atlantic ridge, 2744 meters, the type.

Eastern Atlantic, ca. $27^{\circ}$ N., $14^{\circ}$ W., 2603 meters, one specimen. 
?Eastern Atlantic off the Cape Verde Islands, 2500 meters, one specimen, pelagic.

Eastern Atlantic, ca. $37^{\circ}$ N., $10^{\circ}$ W., 4900 meters, one specimen taken in mid-water together with other pelagic fishes.

Distribution.-Eastern Atlantic from ca. $37^{\circ} \mathrm{N} ., 10^{\circ} \mathrm{W}$. to the Cape Verde Islands, three specimens from deep-abyssal waters and one in 1786 meters (ca. $27^{\circ} \mathrm{N}$., $17^{\circ}$ W.). Mid-Atlantic, one deepabyssal record. Indian Ocean, Arabian Sea, one specimen, 1353 meters.

\section{Length. $-33 \mathrm{~mm}$. and $108-190 \mathrm{~mm}$.}

Remarks.-The type and only known specimen of $P$. procerus, thought to be the young of $P$. apus, was only $33 \mathrm{~mm}$. long and was caught pelagically in a net hauled in 2500 meters off the Cape Verde Islands. Another example taken in mid-water was $150 \mathrm{~mm}$. in standard length, suggesting that the species may be pelagic when adult also.

\section{Holtbyrnia melanocephalus Vaillant}

Bathytroctes melanocephalus Vaillant, 1888, Exp. Sci. Trav. Talis., Poiss., p. 155 (not pl. 11, fig. 3)-Atlantic off North Africa (see footnote, p. 110).

?Bathytroctes innesi Fowler, 1934, Proc. Acad. Nat. Sci. Philadelphia, 85: 252, fig. 14.

Bathytroctes melanocephalus Nobre, 1935, Fauna Marinha Portugal, 1: 519; Fowler, 1936, Bull. Amer. Mus. Nat. Hist., 70: 188; Bertin, 1940, Bull. Mus. Hist. Nat. Paris, 12: 274; Lozano Rey, 1947, Mem. R. Acad. Cien. Madrid, Ser. Cien. Nat., $11: 78$, fig. 12.

? Holtbyrnia innesi Parr, 1937, Bull. Bingham Oceanogr. Coll., 3, (7), p. 6; 1951, Amer. Mus. Nov., 1531: 12.

Holtbyrnia melanocephalus Parr, 1951, loc. cit.

Deep-abyssal record.--?Western Atlantic, ca. $38^{\circ}$ N., $70^{\circ}$ W., 3205 meters, one specimen (innesi).

Distribution.--?Western Atlantic, one deep-abyssal record. Eastern Atlantic, see below.

Length.-195-225 mm.

Remarks.-Vaillant (1888, p. 157) reported four specimens taken off Morocco and Soudan at depths of 1435 meters (Talisman Station 78), 1617 meters (Station 99), 2200 meters (Station 39) and 2600 meters (Station 28). Bertin (1940, p. 274) reported that only two specimens now remain in the Paris museum, those from stations 99 and 28. To the first of these specimens is attached the number given the type by Vaillant (no. 85-167), but since it measures 225 
mm., whereas Vaillant gave the length as $108 \mathrm{~mm}$., Bertin concluded that Vaillant must by error have placed the number on a different specimen and that the type is no longer in the museum. Parr (1951, p. 12), who examined these two specimens, reported that the one bearing the number of the type agrees with the original description but the second specimen differs and has been made the type of a new genus and species, Bellocia vaillanti (family Alepocephalidae). It is now impossible to know to which species belonged the examples from stations 78 and 39 .

\section{Searsia polycoeca Parr}

Searsia polycoeca Parr, 1937, Bull. Bingham Oceanogr. Coll., 3, (7), pp. 11, 15, 19, figs. 1, 5- near Bermuda.

Holtbyrnia (Mentodus) polycoeca Parr, 1951, Amer. Mus. Nov., 1531: 16;

Maul, 1954, Bol. Mus. Mun. Funchal, 7, (17), p. 41, figs. 14-16.

Searsia polycoeca Krefft, 1953, Zool. Anz., 151: 264; Tucker, 1954, Bull. Brit.

Mus. (Nat. Hist.), Zool., 2: 201, 202.

Deep-abyssal record.-Western Atlantic, 2012 meters, the type.

Distribution.-Eastern Atlantic, ca. $10^{\circ} \mathrm{N} ., 21^{\circ} \mathrm{W}$, one specimen, 400-875 meters, closing net; and one from the stomach of an Aphanopus carbo taken in deep water at Madeira. Western Atlantic, one deep-abyssal record.

Length. -48-148 mm. (without caudal).

Remarks.-The species is apparently bathypelagic and probably is not an inhabitant of deep-abyssal waters.

\section{Pellisolus facilis Parr}

Pellisolus facilis Parr, 1951, Amer. Mus. Nov., 1531 : 18, 19-Gulf of Panama, ca. $6^{\circ} \mathrm{N} ., 80^{\circ} \mathrm{W}$.

Pellisolus facilis Tucker, 1954, Bull. Brit. Mus. (Nat. Hist.), Zool., 2: 206.

Deep-abyssal record and distribution.--Eastern Pacific, ?2300 meters, one specimen taken in a pelagic net, with 3600 meters of wire out. Depth to bottom not known.

Length. $-98 \mathrm{~mm}$. (without caudal).

Remarks.-The British Museum collection contains an example of this species but details of its origin have not been published.

\section{Barbantus curvifrons Roule and Angel}

Bathytroctes curvifrons Roule and Angel, 1931, Bull. Inst. Océanogr. Monaco, 581: 6-Gulf of Gascony, ca. $46^{\circ} \mathrm{N} ., 10^{\circ} \mathrm{W}$. 
Bathytroctes curvifrons Roule and Angel, 1933, Rés. Camp. Sci. Monaco, 86: 6, pl. 1, fig. 2; Lozano Rey, 1947, Mem. R. Acad. Cien. Madrid, Ser. Cien. Nat., 11 : 74, pl. 3, fig. 3; Belloc, 1949, Bull. Inst. Océanogr. Monaco, 958: 5 .

Barbantus curvifrons Parr, 1951, Amer. Mus. Nov., 1531 : 18.

Deep-abyssal record and distribution.-Eastern Atlantic, 4500 meters, one specimen caught in a pelagic net.

Length. $-105 \mathrm{~mm}$.

\section{Family GONOSTOMATIDAE}

Apparently all members of this cosmopolitan family are bathypelagic, some being quite abundant at levels higher than those under consideration here. The wide vertical ranges shown by some species are partly due to the fact that many gonostomatid fishes, or their young, make daily vertical migrations and are found in shallower water at night, and partly because some species live at higher levels when young. Unfortunately, it has not been possible to evaluate either the day and night distribution or the ontogenetic migrations of the species discussed below.

Gonostoma denudatum Rafinesque has been omitted, although Norman (1930, p. 282) reported one specimen from a closing net hauled in $2500-2700$ meters (eastern Atlantic, ca. $5^{\circ}$ N., $17^{\circ}$ W.). The species appears to be fairly common above 500 meters, the only deeper records being one specimen at 1180 and three at 2000 meters.

Gonostoma elongatum Günther has also been omitted, although it may occasionally reach the deep-abyssal zone. Its center of distribution seems to be higher.

Gonostoma bathyphilum Vaillant. Table 2 .

Neostoma bathyphilum Vaillant, *1884, Nature, Paris, 22: 184, fig.-Gulf of Gascony, Talisman Station 140 (see footnote on p. 110).

Neostoma bathyphilum Vaillant, 1888, Exp. Sci. Trav. Talis., Poiss., p. 96, pl. 8 , fig. 1 .

Cyclothone bathyphila Goode and Bean, 1895, Ocean. Ichth., p. 100, fig. 118; Jordan and Evermann, 1896, Bull. U. S. Nat. Mus., 47: 582; Fowler, 1936, Bull. Amer. Mus. Nat. Hist., 70: 227, fig. 103.

Cyclothone grandis Collett, 1896, Bull. Soc. Zool. France, 21: 99; Roule and Angel, 1933, Rés. Camp. Sci. Monaco, 86: 24 ; Fowler, 1936, Bull. Amer. Mus. Nat. Hist., 70: 229; Belloc, 1949, Bull. Inst. Océanogr. Monaco, 958: 8 .

Gonostoma bathyphilum Brauer, 1906, Wiss. Ergebn. Deutschen Tiefsee Exp. Valdivia, 15, (1), p. 73; Holt and Byrne, 1906, Rep. Fish. Ireland 1905, 
Sci. Invest., 2: 22; 1907, Trans. Linn. Soc. London, (2), 10, Zool., p. 194; 1913, Rep. Fish. Ireland 1912, Sci. Invest., 1: 11, figs. 3, 4; Zugmayer, 1911, Rés. Camp. Sci. Monaco, 35: 49, pl. 2, fig. 1; Roule, 1919, op. cit., 52: 27; Vaillant, in Roule, 1919, op. cit., p. 130; Nusbaum-Hilarowicz, 1923, op. cit., 65: 17, 77, pl. 3, figs. 3-6, 8; Roule and Angel, 1923, Bull. Inst. Océanogr. Monaco, 429: 4; Norman, 1930, Disc. Rep., 2: 285; Zugmayer, in Roule and Angel, 1933, Rés. Camp. Sci. Monaco, 86: 79; Parr, 1934, Bull. Bingham Oceanogr. Coll., 4, (6), p. 5; 1937, op. cit., 3, (7), p. 41, fig. 17; Beebe, 1937, Zoologica, 22: 201; Bertin, 1939, Bull. Mus. Hist. Nat. Paris, (2), 11: 378; 1940, op. cit., 12: 312; Schroeder, 1940, Copeia, p. 232; Lozano Rey, 1947, Mem. R. Acad. Cien. Madrid, Ser. Cien. Nat., 11: 161, figs. 37, 38; Nybelin, 1948, Göteborgs K. Vetensk. Vitt.-Samh. Handl., (B), 5, (16), p. 18; Smith, 1949, Sea Fishes So. Afr., p. 104, fig. 151; Marshall, 1951, Jour. Mar. Res., 10: 3; 1954, Aspects Deep Sea Biol., pp. 209, 224, 291-292, figs. viii-12, ix-4; Grey, 1955, Fieldiana, Zool., 37: 272.

Gonostoma grande Murray and Hjort, 1912, Depths of Ocean, pp. 101, 604, $612,618,625,627,628,664,665,702,720,739,744$, pl. 2, text figs. 477 , 490, 535; Nusbaum-Hilarowicz, 1923, Rés. Camp. Sci. Monaco, 65: 18, 77, pl. 2, figs. 25, 26, pl. 3, fig. 7, pl. 10, figs. 12-18; Zugmayer, in Roule and Angel, 1933, op. cit., 86: 79.

Gonostoma grandis Barnard, 1925, Ann. So. Afr. Mus., 21 : 143.

?Gonostoma sp. Roule and Angel, 1930, Rés. Camp. Sci. Monaco, 79: 25.

Distribution.-Atlantic from ca. $56^{\circ}$ N., to South Africa (ca. $34^{\circ} \mathrm{S}$.) in the east and from ca. $43^{\circ} \mathrm{N}$. to $17^{\circ} \mathrm{N}$. in the west. Probably a deep-abyssal inhabitant. Depth range 650-5100 meters.

Of more than 130 recorded specimens, at least seventy-five were caught in deep-abyssal waters. There are four closing net records, two in the Gulf of Gascony in 2744-2286 meters (one, $110 \mathrm{~mm}$.) and 2744-1372 meters (one, $85 \mathrm{~mm}$.) and two off South Africa in 1410-1310 meters (three, $80-130 \mathrm{~mm}$.) and 950-850 meters (one, $60 \mathrm{~mm}$.$) .$

\section{Length.-29-180 mm.}

Remarks.-Available evidence suggests that older specimens live principally below 2000 meters. Of the examples from shallower water, only three of the twenty-eight whose lengths were given exceeded $100 \mathrm{~mm}$. $(120,130,165 \mathrm{~mm}$.), whereas below 2000 meters, of the seventeen specimens of known length twelve were between 100 and $180 \mathrm{~mm}$. Only a few authors included the lengths of their specimens.

\section{Cyclothone livida Brauer. Table 3.}

Cyclothone livida Brauer, 1902, Zool. Anz., 25: 279-eastern Atlantic, ca. $9^{\circ} \mathrm{S} ., 9^{\circ} \mathrm{E} ., 2000$ meters. 
Cyclothone livida Brauer, 1906, Wiss. Ergebn. Deutschen Tiefsee Exp. Valdivia, 15, (1), p. 80, pl. 6, fig. 5, text fig. 31; Murray and Hjort, 1912, Depths of Ocean, pp. 612, 676, fig. 493; Pappenheim, 1914, Deutsche SüdpolarExp. 1901-03, 15, Zool., 7: 178; Barnard, 1925, Ann. So. Afr. Mus., 21: 145; Norman, 1930, Disc. Rep., 2: 287; Fowler, 1936, Bull. Amer. Mus. Nat. Hist., 70: 224, fig. 102; Smith, 1949, Sea Fishes So. Afr., p. 103; Marshall, 1951, Jour. Mar. Res., 10: 3.

Distribution.-Eastern and south Atlantic from $28^{\circ}$ N. to $28^{\circ} \mathrm{S}$, $11^{\circ} \mathrm{E}$. to $29^{\circ} \mathrm{W}$. and in ca. $35^{\circ} \mathrm{S} ., 2^{\circ} \mathrm{E}$. At least 251 specimens recorded from twenty-five hauls, of which only six were made above 2000 meters. Extreme range 500-4972 meters.

Brauer did not record the number of specimens per haul, but he did state that of 141 specimens only two were caught above a depth of about 1200 meters. A review of the Valdivia stations shows that only three stations were above 2000 meters, one in 600 , one in 1200 , and one in 1500 meters. Brauer noted that at one station in the Gulf of Guinea thirty specimens were caught in a vertical haul from 3000 meters, while a net on the same line, lowered to only 700 meters, contained no specimens.

On the evidence of the Valdivia catches alone the species would be considered almost restricted to deep-abyssal waters, but both Pappenheim (1914, p. 178) and Norman (1930, p. 287) reported it in some abundance up to 800 meters. However, the total number of specimens known from above 2000 meters is only ninety-seven (not including the Valdivia haul in 1500 meters, number of specimens unknown) and thus most of the known examples were caught below that depth.

\section{Length. $-9-60 \mathrm{~mm}$.}

Remarks. - There is little relationship between size and depth of capture on the basis of known facts, although the shallowest catch, in 500 meters, consisted of two examples only $18-19 \mathrm{~mm}$. long (Pappenheim, 1914). The largest specimen reported, $60 \mathrm{~mm}$., was caught in a haul from 3000 meters, together with nine others, including six "very young" (op. cit.). Norman (1930) recorded ninety specimens ranging in standard length from 20 to $53 \mathrm{~mm}$., in four hauls made in 800-1200 meters. Brauer (1906) gave the length of the Valdivia specimens as $9-39 \mathrm{~mm}$. (without caudal) but did not specify the size of the two examples from 600 meters.

Cyclothone microdon Günther. Tables 4, 5 .

Gonostoma microdon Günther, 1878, Ann. Mag. Nat. Hist., (5), 2: 187--type locality not designated. 
Cyclothone lusca Goode and Bean, 1883, Bull. Mus. Comp. Zool., 10: 221; Jordan, 1887, Rep. U. S. Fish Comm., 1885: 834; Gilbert, 1891, Proc. U. S. Nat. Mus., 13: 449.

Gonostoma microdon Günther, 1887, Rep. Sci. Res. Voy. Challenger, Zool., 22: 175; Alcock, 1889, Ann. Mag. Nat. Hist., (6), 4: 399; 1890, op. cit., (6), 6: 222; Holt and Byrne, 1907, Trans. Linn. Soc. London, (2), 10, Zool., p. 194.

Neostoma quadrioculatum Vaillant, 1888, Exp. Sci. Trav. Talis., Poiss., p. 99, pl. 8, fig. 2.

Gonostoma (Cyclothone) microdon Lütken, ${ }^{*} 1891$, Vidensk. Medd. Dansk naturh. Foren., 1891: 216; 1892, Vidensk. Selsk. Skr. naturv. Math. Afhandl., 7: 280, pl. 2, figs. 4, 5; Holt and Byrne, 1904, Ann. Mag. Nat. Hist., (7), $14: 37,39$.

Cyclothone microdon Goode and Bean, 1895, Ocean. Ichth., pp. 99, 514, fig. 114 ; Jordan and Evermann, 1896, Bull. U. S. Nat. Mus., 47: 582; Gilbert, 1896, Rep. U. S. Comm. Fish, 1893 : 402, 465; Collett, 1896, Rés. Camp. Sci. Monaco, 10: 130; Jordan and Gilbert, 1899, Rep. Fur-seal Invest., 3: 441; Alcock, 1899, Descr. Cat. Indian Deep-sea Fishes, p. 141; Lo Bianco, *1901, Mitt. Zool. Stat. Neapel, 15: 413; *1903, op. cit., 16: 109; 1904, Pelag. Tiefsee-fische Maja, 1: 20 (part), pl. 2, fig. 4; 1909, Mitt. Zool. Stat. Neapel, 19: 714; Ehrenbaum, 1902, Fauna Arct., Fische, 2: 136; Woltereck, 1904, Zool. Jahrb., 7, Suppl., p. 347; Lönnberg, 1905, Wiss. Ergebn. Schwed. Südpol Exp., 5, (6), p. 65; Holt and Byrne, 1906, Rep. Fish. Ireland 1905, Sci. Invest., $2: 22 ; 1913$, Rep. Fish. Ireland 1912, Sci. Invest., 1: 12, fig. 5; Brauer, 1906, Wiss. Ergebn. Deutschen Tiefsee Exp. Valdivia, 15, (1), p. 82, pl. 6, fig. 4, text fig. 32; Evermann and Goldsborough, 1907, Bull. U. S. Bur. Fish., 26:271; Mazzarelli, *1909, Riv. Mens. Pesc. Idrobiol., 4, (11); *1910, op. cit., 5, (10-12); Fage, 1910, Ann. Inst. Océanogr. Paris, 1, (7), p. 6; Zugmayer, 1911, Rés. Camp. Sci. Monaco, 35: 43; in Roule and Angel, 1933, op. cit., 86: 80; Gilbert and Burke, 1912, Bull. Bur. Fish., 30: 35; Murray and Hjort, 1912, Depths of Ocean, pp. $86,101-103,108,118,126,604,612,618,622,624-627,659,664,665$, $676,677,681,699,718,739-741,771$, pl. 1, text figs. $473,474,476,493$, 528, 530, c; Weber, 1913, Monogr. Siboga Exp., 57: 18; Weber and de Beaufort, 1913, Fishes Indo-Austr. Arch., 2: 126, fig. 46; Regan, 1913, Trans. Roy. Soc. Edinburgh, 49: 232; 1916, Rep. Brit. Antarctic (Terra Nova) Exp. 1910, Zool., 1 : 137, pl. 5, fig. 5; Pappenheim, 1914, Deutsche Südpolar-Exp. 1901-1903, 15, Zool., 7: 178; Roule, 1919, Rés. Camp. Sci. Monaco, 52: 27; Vaillant, in Roule, 1919, op. cit., p. 130; Jespersen and Tåning, 1919, Vidensk. Medd. Dansk naturh. Foren., 70 : 217; 1926, Rep. Danish Oceanogr. Exp. 1908-1910, 2, (A 12), p. 7; Roule and Angel, 1921, Bull. Inst. Océanogr. Monaco, 397: 2; 1924, op. cit., 451: 3; 1930, Rés. Camp. Sci. Monaco, 79: 26, pl. 2, fig. 28; Barnard, 1925, Ann. So. Afr. Mus., 21 : 146; Breder, 1927, Bull. Bingham Oceanogr. Coll., 1, (1), p. 44; Beebe, 1929, Zoologica, 12: 13; 1933, Proc. Nat. Acad. Sci., Washington, 19: 180; 1937, Zoologica, 22: 201; Norman, 1930, Disc. Rep., 2: 287; 1937, Rep. B.A.N.Z. Antarctic Res. Exp. 1929-1931, 1: 82; Angel and Verrier, 1931, Ann. Inst. Océanogr. Paris, 10 : 123; Gregory, 1933, Trans. Amer. Phil. Soc., 23: 161, fig. 54; Parr, 1934, Bull. Bingham Oceanogr. Coll., 4, 
(6), pp. 5, 11, figs. $3,12,13 ; 1937$, op. cit., 3, (7), p. 44; Fowler, 1936, Bull. Amer. Mus. Nat. Hist., 70: 225, 1204; Belloc, 1938, Rev. Trav. Pêches Marit., 11, (3), p. 285, fig. 5; Bertin, 1939, Bull. Mus. Hist. Nat. Paris, 11 : 378 ; 1940, op. cit., 12: 312; Chapman, 1940, Occ. Pap. British Columbia Prov. Mus., 2: 11; Longley and Hildebrand, 1941, Publ. Carnegie Inst. Washington, 535: 15; Tortonese, 1941, Riv. Sci. Nat. Natura, 32: 76; Clemens and Wilby, 1946, Bull. Fish. Res. Bd. Canada, 68: 105, fig. 53; Lozano Rey, 1947, Mem. R. Acad. Cien. Madrid, Ser. Cien. Nat., 11 : 167, pl. 5, fig. 1, text fig. 41; Nybelin, 1948, Göteborgs K. Vetensk. Vitt.-Samh. Handl., (B), 5, (16), p. 20, pl. 3, fig. 1; Jensen, 1948, Skr. Univ. Zool. Mus. København, 9: 107, chart 7; Smith, 1949, Sea Fishes So. Afr., p. 103, fig. 149; Schmidt, 1950, Akad. Sci. U.S.S.R. Trans. Pac. Comm., 6: 54; Misra, 1950, Rec. Indian Mus., 45: 413; 1953, op. cit., 50 : 398; Marshall, 1951, Jour. Mar. Res., 10: 3, 8, 11; 1954, Aspects Deep Sea Biol., pp. 109, 223, figs. v-14, ix-4; Koumans, 1953, Temminckia, 9: 182; Günther and Deckert, *1953, Z. Morph. Ökol., 42:1; Mukhacheva, *1954, Trudy Inst. okean., 11: 206; Grey, 1955, Fieldiana, Zool., $37: 272$.

Cyclothone (Gonostoma) microdon Lütken, 1898, Danish Ingolf Exp., 2, (1), p. 9.

Cyclothone microdon pygmaea Jespersen and Tåning, 1926, Rep. Danish Oceanogr. Exp. 1908-1910, 2, (A 12), p. 7, figs. 5-7, 9, 12; Norman, 1930, Disc. Rep., 2: 288; Sanzo, 1931, Monogr. Fauna Flora Golfo Napoli, 38: 78, 80, figs. 57, 58; Tortonese and Trotti, 1949, Atti Accad. Ligure Sci. Lett., 6, (1), p. 36 .

Distribution.-World-wide, including high latitudes in north Atlantic and Antarctic seas, but not in water of negative temperatures. Perhaps replaced in the eastern and north Pacific by $C$. acclinidens. Not reported by Australian or Japanese authors, perhaps because the species inhabits depths beyond the normal range of local fishing vessels.

Of more than 300 hauls containing $C$. microdon made throughout the world, scarcely fifty have been shallower than 1000 meters, about 100 between 1000 and 2000 meters, and about 170 below 2000 meters. The Monaco expeditions carried on the greatest amount of deep-abyssal mid-water fishing, and although we know the number of specimens per haul at only a few of these stations, at several of them (2500-4000 meters) "numerous" examples were caught. Lönnberg $(1905$, p. 65) also reported numerous specimens from two hauls made in 2700 and 2500 meters. The normal habitat was placed between about 550 and 1450 meters by Holt and Byrne (1913, p. 12) and by Murray and Hjort (1912, p. 619), both conclusions based on hauls made chiefly in depths above 2000 meters.

The following fourteen hauls containing $C$. microdon were made with closing nets. 


\section{Eastern Atlantic}

Ca. $48^{\circ}$ N., $20^{\circ}$ W., 2200 meters. Six young specimens in this and the following haul (Collett, 1896).

Ca. $47^{\circ}$ N., $22^{\circ}$ W., 1300 meters (Collett, 1896).

\section{Mid-Allantic}

Ca. $36^{\circ}$ N., $43^{\circ}$ W., 4500-1500 meters. Ten specimens (Murray and Hjort, 1912).

Ca. $36^{\circ}$ N., $43^{\circ}$ W., $1350-450$ meters. Twenty-seven specimens (Murray and Hjort, 1912).

Ca. $47^{\circ} \mathrm{N} ., 43^{\circ} \mathrm{W} ., 950-525$ meters. (?)specimens (Murray and Hjort, 1912).

\section{Off South Africa}

Ca. $33^{\circ}$ S., $15^{\circ}$ E., $2500-2000$ meters. Twenty-three specimens, 30-60 mm. (Norman, 1930). 1906).

Ca. $42^{\circ}$ S., $14^{\circ}$ E., $1500-900$ meters. One specimen (Brauer,

South Atlantic

Ca. $38^{\circ}$ S., $22^{\circ}$ W., 2000-1800 meters. Nine specimens, 26-54 mm. (Norman, 1930).

Ca. $53^{\circ}$ S., $35^{\circ}$ W., $1275-1025$ meters. Twenty-two specimens, 34-68 mm. (Norman, 1930).

Ca. $43^{\circ}$ S., $46^{\circ}$ W., $750-500$ meters. One specimen, $27 \mathrm{~mm}$. (Norman, 1930).

\section{Western Pacific}

Ca. $1^{\circ}$ S., $118^{\circ}$ E., $500-345$ meters. Two specimens, 18 and 25 $\mathrm{mm}$. (Koumans, 1953).

Ca. $2^{\circ}$ S., $121^{\circ}$ E., 500-340 meters. One specimen, $20 \mathrm{~mm}$. (Koumans, 1953).

\section{North Indian}

Ca. $3^{\circ}$ N., $63^{\circ}$ E., 1000-555 meters. Three specimens, 20-28 mm. (Koumans, 1953).

\section{Antarctic}

Ca. $60^{\circ}$ S., $51^{\circ}$ W., $1100-1000$ meters. Three specimens, 55-60 mm. (Norman, 1930). 
It is probably correct to assume that the species inhabits deepabyssal waters. Figures shown in Tables 4 and 5 suggest that the depth of its maximum abundance is somewhat less (1000-1600 meters) and may vary slightly with latitude. There is also some evidence that a smaller center of distribution may exist in the deepabyssal zone.

The Mediterranean form, C. microdon pygmaea Jespersen and Tåning, inhabits shallower water (maximum occurrence of adult about 1000 meters in summer, $700-800$ meters in winter).

Length. $-6-76 \mathrm{~mm}$.

Remarks.-Holt and Byrne (1913, p. 15) noted the almost constant presence of small numbers of $C$. microdon in nets fastened to the back of the trawl in hauls taken in about 900-1300 meters and were inclined to believe that the fishes were actually present near the bottom, rather than that their capture in these nets was due to their occurrence in such great numbers at lesser depths that a few inevitably were caught as the nets were being pulled up. The presence of the species near the bottom in these depths may possibly be explained by the fact that it makes daily vertical migrations and thus may drift over lesser depths during the night.'

Murray and Hjort (1912, p. 621) wrote: “... it is perfectly clear that the smaller sizes are met with much higher up than the larger ones, which latter are mainly to be found at a depth of 1500 m." The same authors also noted that the average size was greater in their more northerly hauls. Data from other sources support the evidence given by these authors and also reveal the fact that in cold seas large specimens are found in shallower water.

Eastern and north Pacific records have been omitted from Tables 4 and 5 , both because of the small number of records and because these specimens may prove to be acclinidens. The one mid-Pacific record has been omitted also.

\section{Cyclothone acclinidens Garman. Table 6.}

Cyclothone acclinidens Garman, 1899, Mem. Mus. Comp. Zool., 24: 247, pI. J, fig. 4 -eastern Pacific (type locality not designated).

Cyclothone acclinidens Gierse, 1904, Morph. Jahrb., 32: 602, pls. 14-16; Brauer, 1906, Wiss. Ergebn. Deutschen Tiefsee Exp. Valdivia, 15, (1), p. 85, pl. 6, fig. 1, text fig. 34; Lloyd, 1909, Mem. Indian Mus., 2: 151; Murray and Hjort, 1912, Depths of Ocean, pp. 612, 676, fig. 493; Weber,

${ }^{1}$ Young only? See Marshall, 1951, p. 12. 
1913, Monogr. Siboga Exp., 57: 19; Weber and de Beaufort, 1913, Fishes Indo-Austr. Arch., 2: 127; Pappenheim, 1914, Deutsche Südpolar-Exp. 1901-1903, 15, Zool., 7 : 180; Barnard, 1925, Ann. So. Afr. Mus., 21 : 147; Townsend and Nichols, 1925, Bull. Amer. Mus. Nat. Hist., 52: 11; Borodin, 1928, Bull. Vanderbilt Mar. Mus., 1, (1), p. 11; Beebe, 1929, Zoologica, 12: 13; Norman, 1930, Disc. Rep., 2: 288; 1939, Sci. Rep. John Murray Exp. 1933-34, 7, (1), p. 18; Parr, 1931, Bull. Bingham Oceanogr. Coll., 2, (4), p. 11; Fowler, 1936, Bull. Amer. Mus. Nat. Hist., 70: 226; Smith, 1949, Sea Fishes So. Afr., p. 103; Misra, 1950, Rec. Indian Mus., 45: 413; 1953, op. cit., 50 : 397, fig. 19, a; Marshall, 1951, Jour. Mar. Res., 10: 3; Follett, 1952, Proc. California Acad. Sci., 27: 409; Koumans, 1953, Temminckia, 9: 183.

Distribution.-Eastern Atlantic, Azores to Cape Point. MidAtlantic, $0^{\circ} 46^{\prime}$ N., $18^{\circ} 59^{\prime} \mathrm{W}$. to $17^{\circ} 28^{\prime}$ N., $29^{\circ} 42^{\prime}$ W. Western Atlantic off New York (misidentification?). South Atlantic, ca. $55^{\circ}$ S., $18^{\circ} \mathrm{W}$. and $56^{\circ} \mathrm{S} ., 14^{\circ} \mathrm{E}$. Tropical Indian Ocean, northeast coast of Africa, Gulf of Aden, Arabian Sea, Bay of Bengal, to $26^{\circ}$ S., $93^{\circ} \mathrm{E}$. and $10^{\circ} \mathrm{S} ., 97^{\circ} \mathrm{E}$. Western Pacific around the Dutch East Indies. Eastern Pacific from $0^{\circ} 16^{\prime}$ to $37^{\circ} \mathrm{N}$. and west to $138^{\circ} \mathrm{W}$.

C. acclinidens is an abundant species. It has been taken at only about fifteen stations above 1000 meters. Of around ninety hauls made below that depth, half were deeper than 2000 meters, but in most cases the number of specimens taken in each haul was not published, and so it has been impossible to determine the center of distribution. The species probably inhabits deep-abyssal waters, although it may prefer higher levels. It has been reported in three hauls made with closing nets, in the Celebes Sea in 1100-790 meters (two specimens) and in the Arabian Sea in 1045-984 meters (one specimen) and 984-430 meters (seventeen specimens). Extreme depth range $224-4417$ meters.

\section{Length. - 10-50 mm. (without caudal).}

Remarks. - C. acclinidens is very close to $C$. microdon and the two species have probably been confused at times. Their vertical distribution appears to be almost identical. If they are actually distinct, or if they represent races of the same species, a difference in horizontal distribution would be expected. However, both species have been reported in the same nets by several authors. Although a study of the literature suggests that acclinidens is absent or scarce in the northern and western Atlantic and in the Antarctic, and that it perhaps replaces microdon in the eastern and northern Pacific, a further understanding of the two species will require the study of a large series of specimens from all over the world. 


\section{Cyclothone obscura Brauer}

Cyclothone obscura Brauer, 1902, Zool. Anz., 25: 280-Indian Ocean, ca. $4^{\circ} \mathrm{S}$, $48^{\circ}$ E., 2000 meters.

Cyclothone obscura Brauer, 1906, Wiss. Ergebn. Deutschen Tiefsee Exp. Valdivia, 15, (1), p. 88, pl. 6, fig. 3, text fig. 35; Pappenheim, 1914, Deutsche Südpolar-Exp. 1901-1903, 15, Zool., 7: 181; Barnard, 1925, Ann. So. Afr. Mus., 21 : 147; Norman, 1930, Disc. Rep., 2: 288; Fowler, 1936, Bull. Amer. Mus. Nat. Hist., 70: 227; Smith, 1949, Sea Fishes So. Afr., p. 103; Misra, 1950, Rec. Indian Mus., 45: 414; 1953, op. cit., 50: 397, fig. 15, $a$; Marshall, 1954, Aspects Deep Sea Biol., p. 290.

Distribution.-Eastern Atlantic, ca. $17^{\circ} \mathrm{N}$. to $31^{\circ} \mathrm{S}$. and $7^{\circ} \mathrm{E}$. to $29^{\circ} \mathrm{W}$. Indian Ocean, ca. $9^{\circ}-10^{\circ} \mathrm{S}$, $53^{\circ}-97^{\circ} \mathrm{E}$. Forty-four specimens known from twenty-four hauls, of which only three were shallower than 2000 meters (one in 800 , two in 1500 meters). Depth range $800-5248$ meters.

Length. $-12-60 \mathrm{~mm}$. (without caudal).

\section{Family BATHYLACONIDAE}

\section{Bathylaco nigricans Goode and Bean}

Bathylaco nigricans Goode and Bean, 1895, Ocean. Ichth., p. 57, fig. 69Atlantic off the Virgin Islands.

Bathylaco nigricans Jordan and Evermann, 1896, Bull. U. S. Nat. Mus., 47: 540; Parr, 1948, Copeia, p. 52, figs. 1, 2.

Deep-abyssal records and distribution.-Western Atlantic, 4370 meters, the type.

Eastern Pacific off Colombia, 2200 meters, one specimen taken in a pelagic net, with 3500 meters of wire out.

Length.-209-215 mm. (without caudal).

Remarks. -No definite conclusions can be drawn concerning the habitat, but the species probably lives on or near the bottom.

Goode and Bean (1895, p. 58) listed the type from "off Santa Cruz at a depth of 2393 fathoms," and stated that it was taken by the Blake. Jordan and Evermann (1896, p. 540) gave the type locality as "off Santa Cruz, Mexico," and Jordan, Evermann and Clark (1930, p. 164) as "Gulf of Mexico; off Santa Cruz." Smith (1888, p. 966) listed only one Blake station in a depth of 2393 fathoms, Agassiz Station no. 121, Jan. 2, 1879, $17^{\circ} 56^{\prime} 55^{\prime \prime}$ N., $64^{\circ} 54^{\prime} 15^{\prime \prime} \mathrm{W}$., between St. Thomas and Santa Cruz. This locality is near the Virgin Islands; the "Santa Cruz" referred to is undoubtedly the island more commonly known as St. Croix. 


\section{Family HARPADONTIDAE ${ }^{1}$}

The family contains two genera, Harpadon Le Sueur, from relatively shallow water in the Indo-Pacific, and Bathysaurus Günther, with two species apparently confined to deep-abyssal waters, one of them also inhabiting the abyssal plain below 3660 meters. Both were originally described by Günther from material collected by the Challenger and are widely distributed in the Atlantic and Pacific oceans.

\section{Bathysaurus ferox Günther}

Bathysaurus ferox Günther, 1878, Ann. Mag. Nat. Hist., (5), 2: 182-Pacific off New Zealand, ca. $40^{\circ} \mathrm{S} ., 177^{\circ}$ E., 2012 meters.

Bathysaurus agassizii Goode and Bean, 1883, Bull. Mus. Comp. Zool., 10: 215; Vaillant, 1888, Exp. Sci. Trav. Talis., Poiss., p. 139, pl. 10, fig. 1.

Bathysaurus ferox Günther, 1887, Rep. Sci. Res. Voy. Challenger, Zool., 22: 181, pl. 46, fig. A; Vaillant, 1888, Exp. Sci. Trav. Talis., Poiss., p. 385; Goode and Bean, 1895, Ocean. Ichth., p. 58, figs. 55, 56; Jordan and Evermann, 1896, Bull. U. S. Nat. Mus., 47: 539; Murray and Hjort, 1912, Depths of Ocean, pp. 121, 396, fig. 103, $a$; Gilchrist and von Bonde, 1924, Rep. Fish. Mar. Biol. Surv. So. Afr., 3, (7), p. 8; Barnard, 1925, Ann. So. Afr. Mus., 21: 228; Koefoed, 1927, Rep. Sci. Res. M. Sars No. Atl. Deep-sea Exp. 1910, 4, (1), p. 62, pl. 4, fig. 4; Fowler, 1936, Bull. Amer. Mus. Nat. Hist., 70 : 345, fig. 161; Schroeder, 1940, Copeia, p. 233; Lozano Rey, 1947, Mem. R. Acad. Cien. Madrid, Ser. Cien. Nat., 11: 382, pl. 6, fig. 3; Smith, 1949, Sea Fishes So. Afr., p. 114, fig. 179.

Deep-abyssal records. - Mid-Atlantic on the mid-Atlantic ridge, ca. $45^{\circ}$ N., $25^{\circ}$ W., 3120 meters, one specimen.

Eastern Atlantic, ca. $34^{\circ} \mathrm{N} ., 33^{\circ} \mathrm{W} ., 2615$ meters, one specimen. Eastern Atlantic, ca. $27^{\circ} \mathrm{N} ., 14^{\circ} \mathrm{W} ., 2603$ meters, one specimen. Eastern Atlantic off Morocco, 2200, 2055 meters, two specimens. Off South Africa, ca. $33^{\circ}$ S., $16^{\circ}$ E., 2560 meters, one specimen. Western Atlantic, ca. $40^{\circ} \mathrm{N}$., $68^{\circ} \mathrm{W}$., 2250 meters, one specimen.

Western Atlantic, ca. $39^{\circ}$ N., $69^{\circ}-70^{\circ}$ W., 2022, 1977 meters, two specimens.

Pacific off New Zealand, 2012 meters, the type.

Distribution.-Eastern Atlantic, $27^{\circ}-50^{\circ}$ N., $8^{\circ}-33^{\circ}$ W., four deep-abyssal specimens and three in 1797 meters. Mid-Atlantic, one deep-abyssal specimen. South Africa off Cape Point and Table Bay, one deep-abyssal specimen and three in 1145-1098 meters. Western Atlantic, $33^{\circ}-40^{\circ} \mathrm{N}$., $68^{\circ}-76^{\circ} \mathrm{W}$., three deep-abyssal specimens

${ }_{1}^{1}$ The classification of iniomid fishes used here is that proposed by Dr. Robert R. Harry (MS.). 
and three in 1813, 1799, and 1183 meters. Southwestern Pacific, one deep-abyssal specimen. Found principally in deep-abyssal waters.

Length. - 216-673 $\mathrm{mm}$.

\section{Bathysaurus mollis Günther}

Bathysaurus mollis Günther, 1878, Ann. Mag. Nat. Hist., (5), 2: 182-type locality not stated, designated as Challenger Station 237, Pacific off Japan, ca. $34^{\circ} \mathrm{N} ., 140^{\circ}$ E., 3429 meters (locality of adult specimen figured in Günther, 1887, pl. 46, fig. B).

Bathysaurus mollis Günther, 1887, Rep. Sci. Res. Voy. Challenger, Zool., 22: 183, pl. 46, fig. B; Vaillant, 1888, Exp. Sci. Trav. Talis., Poiss., p. 385; Vaillant, in Roule, 1919, Rés. Camp. Sci. Monaco, 52: 130; Goode and Bean, 1895, Ocean. Ichth., p. 59; Townsend and Nichols, 1925, Bull. Amer. Mus. Nat. Hist., 52: 10, pl. 1; Koefoed, 1927, Rep. Sci. Res. M. Sars No. Atl. Deep-sea Exp. 1910, 4, (1), p. 62; Fowler, 1936, Bull. Amer. Mus. Nat. Hist., 70: 346, fig. 162; Nybelin, 1951, Rep. Swedish Deep-sea Exp. 1947-1948, Zool., 2, (1), p. 13.

Bathysaurus obtusirostris Vaillant, 1888, Exp. Sci. Trav. Talis., Poiss., p. 136, pl. 10, fig. 2, pl. 14, fig. 3 .

Deep-abyssal records and distribution.--Eastern Atlantic, ca. $39^{\circ}$ N., $17^{\circ} \mathrm{W} ., 4360$ meters, one specimen.

Eastern Atlantic, ca. $29^{\circ}$ N., $17^{\circ}$ W., 4267-4255 meters, one specimen.

Eastern Atlantic off Cape Verde, 3655 meters, one specimen.

Eastern Atlantic, ca. $34^{\circ}$ N., $33^{\circ}$ W., 2615 meters, one specimen.

Eastern Pacific off Lower California, 3219 meters, one specimen.

South Pacific, ca. $22^{\circ}$ S., $150^{\circ}$ W., 4361 meters, one specimen.

Western Pacific, 3429 meters, the type.

Restricted to depths below 2000 meters as far as known, and also found on the abyssal plain. Seven specimens reported.

Length. $-241-600 \mathrm{~mm}$.

\section{Family CHLOROPHTHALMIDAE}

Of the two genera contained in this family, Bathysauropsis is monotypic and probably deep-abyssal.

\section{Bathysauropsis gracilis Günther}

Chlorophthalmus gracilis Günther, 1878, Ann. Mag. Nat. Hist., (5), 2: 182type locality not stated, designated as Challenger Station 300, Pacific off 
Juan Fernandez, ca. $33^{\circ}$ S., $78^{\circ}$ W., 2515 meters (locality of specimen figured in Günther, 1887, pl. 49, fig. A).

Chlorophthalmus gracilis Günther, 1887, Rep. Sci. Res. Voy. Challenger, Zool.,

22: 194, pl. 49, fig. A; Goode and Bean, 1895, Ocean. Ichth., p. 511;

Gilchrist, 1906, Mar. Invest. So. Afr., 4: 165; Thompson, *1916, Prov.

Cape Good Hope Mar. Biol. Rep., 3: 82; Gilchrist and von Bonde, 1924,

Rep. Fish. Mar. Biol. Surv. So. Afr., 3, (7), p. 7.

Bathysauropsis gracilis Regan, 1911, Ann. Mag. Nat. Hist., (8), 7: 127;

Barnard, 1925, Ann. So. Afr. Mus., 21: 230; Parr, 1928, Bull. Bingham

Oceanogr. Coll., 3, (3), p. 18; Smith, 1949, Sea Fishes So. Afr., p. 115, pl. 6, fig. 185 .

Deep-abyssal records.-South Atlantic, ca. $32^{\circ}$ S., $13^{\circ}$ W., 2606 meters, one specimen.

Off South Africa, ca. $33^{\circ}$ S., $16^{\circ}$ E., 2231, 2195 meters, one and an unstated number of specimens (two hauls).

South Pacific off Juan Fernandez, 2515 meters, three specimens.

South Pacific off New Zealand, 2012 meters, one specimen.

Distribution.--South Atlantic, one deep-abyssal specimen. Off Cape Point and Table Bay, South Africa, two deep-abyssal records and at least fifteen specimens in 869 and 1098-1802 meters. South Pacific, two deep-abyssal records.

Length.-102-320 mm.

\section{Family BATHYPTEROIDAE}

Bathypteroid fishes are all found in deep water and, except for one or two young specimens taken pelagically, seem always to be caught in bottom-fishing appliances. Furthermore, as stated by Parr $(1928$, p. 3), the structure of these fishes suggests a benthic existence. Of the eighteen species of Bathypterois described, eight are known from deep-abyssal waters but the only one taken in any abundance, $B$. dubius, apparently prefers shallower water. It should be noted that Nybelin (1951, pp. 15, 24, 25) reported from the north Atlantic as Bathypterois sp. eleven specimens taken at a depth of $5610-5600$ meters $\left(9^{\circ} 38^{\prime} \mathrm{N} ., 26^{\circ} 20^{\prime} \mathrm{W}\right.$.), two at $5000-5025$ meters $\left(43^{\circ} 40^{\prime} \mathrm{N} ., 18^{\circ} 45^{\prime} \mathrm{W}.\right)$, and six at $4590-4600$ meters $\left(40^{\circ}\right.$ $33^{\prime}$ N., $35^{\circ} 24^{\prime}$ W. $)$.

Bruun (1952, in litt.; 1955, pl. 4) has written that one specimen of Benthosaurus was caught by the Galathea Expedition in the Indian Ocean between Madagascar and East Africa, and specimens presumably of the same genus have been seen and photographed in the Mediterranean at depths of 2347 and 2132 meters (MacLeish, 1954; Edgerton, 1955). 


\section{Benthosaurus grallator Goode and Bean}

Benthosaurus grallator Goode and Bean, 1886, Bull. Mus. Comp. Zool., 12 : 168-Gulf of Mexico, 3384 meters.

Benthosaurus grallator Goode and Bean, 1895, Ocean. Ichth., p. 62, fig. 73; Jordan and Evermann, 1896, Bull. U. S. Nat. Mus., 47: 543; 1900, op. cit., fig. 237; Murray and Hjort, 1912, Depths of Ocean, pp. 396, 686, fig. 501, c, 502; Koefoed, 1927, Rep. Sci. Res. M. Sars No. Atl. Deep-sea Exp. 1910, 4, (1), p. 64; Parr, 1928, Bull. Bingham Oceanogr. Coll., 3, (3), p. 23; Fowler, 1936, Bull. Amer. Mus. Nat. Hist., 70: 360, fig. 172.

Skagerakia nilssoni Nybelin, 1946, Ark. Zool., 38B, (2), p. 4, figs. 3, 4; 1948, Göteborgs K. Vetensk. Vitt.-Samh. Handl., (B), 5, (16), p. 32, pl. 2, fig. 3 , pl. 3, fig. 3 .

Deep-abyssal records and distribution.-Western Atlantic, Gulf of Mexico, 3384 meters, one specimen.

Western Atlantic, ca. $39^{\circ}$ N., $70^{\circ} \mathrm{W} ., 2810$ meters, one specimen.

Western Atlantic, Gulf of Mexico, 2104-2194 meters, three specimens (Oregon Station 1303, $28^{\circ} 47^{\prime}$ N., $87^{\circ} 50^{\prime}$ W., May 26, 1955).

Eastern Atlantic, ca. $34^{\circ}$ N., $33^{\circ}$ W., 2865 meters, two specimens.

Eastern Atlantic, ca. $35^{\circ}$ N., $8^{\circ}$ W., 2150-2300 meters, one specimen.

Length.-154-346 mm. (without caudal).

Remarks.-Three recently caught specimens from the Gulf of Mexico show eastern and western Atlantic specimens to be the same. Mr. Robert Kanazawa, of the U. S. National Museum, has also examined one of the types (not the figured specimen), has compared it with the photograph of Skagerakia nilssoni Nybelin and has written (in litt., 1955) that the general appearance and profile are similar, the lateral line turns up toward the upper caudal lobe, which is larger than the lower (exclusive of the prolonged ray), and the dorsal count is 12, anal 13. A careful comparison of all published accounts has revealed no constant differences between western and eastern Atlantic examples.

\section{Bathypterois dubius Vaillant}

Bathypterois dubius Vaillant, 1888, Exp. Sci. Trav. Talis., Poiss., p. 124, pl. 9, fig. 1, pl. 12, fig. 4, pl. 14, fig. 4, pl. 15, fig. 4-Atlantic off North Africa, Canaries, Azores, type locality not stated $^{1}$ (see footnote, p. 110).

${ }^{1}$ Bertin and Estève $(1951$, p. 9) reported that the type specimen, in the Paris Museum, was taken at Talisman Station 128. Vaillant (1888, p. 133), who did not specify the type locality, did not include Station 128 in his listing under $B$. dubius, nor in his list on pages $32-58$. 
Bathypterois longifilis Carus, 1893, Prodr. Faunae Medit., 2: 562.

Bathypterois dubius Goode and Bean, 1895, Ocean. Ichth., p. 64, fig. 74; Collett, 1896, Rés. Camp. Sci. Monaco, 10 : 105, pl. 4, fig. 19; Koehler, 1896, Ann. Univ. Lyon, 26: 507; Holt and Byrne, 1906, Rep. Fish. Ireland 1905, Sci. Invest., 2: 25; Zugmayer, 1911, Rés. Camp. Sci. Monaco, 35: 16; Murray and Hjort, 1912, Depths of Ocean, pp. 80, 396, 686, figs. 57, $b, 266,501, b$; Roule, 1919, Rés. Camp. Sci. Monaco, 52: 34; Vaillant, in Roule, 1919, op. cit., p. 130; Koefoed, 1927, Rep. Sci. Res. M. Sars No. Atl. Deep-sea Exp. 1910, 4, (1), p. 63; Parr, 1928, Bull. Bingham Oceanogr. Coll., 3, (3), p. 26; Roule and Angel, 1933, Rés. Camp. Sci. Monaco, 86: 31; Fowler, 1936, Bull. Amer. Mus. Nat. Hist., 70: 356, fig. 169; Lozano Rey, 1947, Mem. R. Acad. Cien. Madrid, Ser. Cien. Nat., 11: 397, fig. 119; Pardi, 1951, Monit. Zool. Ital., 58: 125, fig.; Bertin and Estève, 1951, Cat. Types Poiss., 6: 9; Tucker, 1954, Bull. Brit. Mus. (Nat. Hist.), Zool., 2: 192; Marshall, 1954, Aspects Deep Sea Biol., pp. 249-250, fig. ix-15.

Deep-abyssal record.-Eastern Atlantic, ca. $39^{\circ}$ N., $26^{\circ}$ W., 1940 meters, one specimen.

Distribution.-Eastern Atlantic from off southwestern Ireland to ca. $16^{\circ} \mathrm{N}$. and west to $33^{\circ} \mathrm{W}$., more than one hundred specimens known in 800-1805 meters and one taken from deep-abyssal waters. Mediterranean, two specimens, one taken at Messina, the second in the Tyrrhenian Sea below 300 meters; a third example, from the Ionian Sea, reported as $B$. longifilis Günther, probably belongs to B. dubius (Pardi, 1951, p. 125). Probably only accidental in the deep-abyssal zone.

Length.-116 (standard)-260 mm.

\section{Bathypterois pectoralis Garman}

Bathypterois pectoralis Garman, 1899, Mem. Mus. Comp. Zool., 24 : 257, pl. K, fig. 1 -off the Galapagos Islands to ca. $23^{\circ} \mathrm{N}$., $108^{\circ} \mathrm{W}$. (type locality not stated).

Bathypterois pectoralis Parr, 1928, Bull. Bingham Oceanogr. Coll., 3, (3), p. 26, footnote.

Deep-abyssal records.-Eastern Pacific, ca. $3^{\circ}$ N., $82^{\circ}$ W., 2070 meters, an unstated number of specimens from one haul.

Eastern Pacific, ca. $7^{\circ}$ N., $79^{\circ}$ W., 1865 meters, an unstated number of specimens from one haul.

Distribution.-Eastern Pacific only, from off the Galapagos Islands $\left(0^{\circ} 4^{\prime} \mathrm{S} ., 90^{\circ} \mathrm{W}\right.$.) to Mexico (ca. $23^{\circ} \mathrm{N}$., $108^{\circ} \mathrm{W}$.), an unstated number of specimens from four hauls, two of them deep-abyssal, one in 1820 meters and one in 1619 meters.

Length.-?-254 $\mathrm{mm}$. 


\section{Bathypterois antennatus Gilbert}

Bathypterois antennatus Gilbert, 1905, Bull. U.S. Fish Comm., 23: 590, fig. 235

-Pacific off Bird Island, Hawaiian Islands, 571-1463 meters.

Bathypterois atricolor antennatus Parr, 1928, Bull. Bingham Oceanogr. Coll., 3, (3), p. 31; Matsubara, 1954, Jap. Jour. Ichth., 3: 62, fig. 1.

Bathypterois longifilis Fowler, 1928, Mem. Bishop Mus., 10:66 (part).

Bathypterois antennatus Böhlke, 1953, Stanf. Ichth. Bull., 5: 17.

Deep-abyssal record.-Hawaiian Islands near Kauai, 1829-2403 meters, one specimen.

Distribution.-Mid-Pacific off the Hawaiian Islands, one deepabyssal specimen and one in 571-1463 meters. Western Pacific off Japan (Mie Prefecture), one specimen in ca. 258 meters.

Length. - 165-200 mm.

Remarks. - B. atricolor indicus Brauer (1906, p. 144), known from one specimen taken at a depth of 1644 meters off the northeast coast of Africa, is synonymized with this species by Parr (1928, p. 31).

\section{Bathypterois phenax Parr}

Bathypterois atricolor phenax Parr, 1928, Bull. Bingham Oceanogr. Coll., 3, (3), p. 31-near the Bahama Islands, 1645-1729 meters.

Bathypterois atricolor phenax Shanklin, 1934, Acta Zool. Stockholm, 15: 409, figs. 6-8; 1935, Phil. Trans. London, (B), 224: 361, figs. 12-19.

Deep-abyssal record.-Gulf of Mexico, 2104-2194 meters, two specimens (Oregon Station 1303, $28^{\circ} 47^{\prime}$ N., $87^{\circ} 50^{\prime}$ W., May 26, 1955).

Distribution.-Western Atlantic only, two deep-abyssal specimens from the Gulf of Mexico and two specimens in 1645-1729 meters from near the Bahamas.

Length. -86-162 mm. (without caudal).

\section{Bathypterois longipes Günther}

Bathypterois longipes Günther, 1878, Ann. Mag. Nat. Hist., (5), 2: 184 Atlantic off Montevideo, ca. $36^{\circ} \mathrm{S} ., 46^{\circ} \mathrm{W} ., 4846$ meters.

?Bathypterois longicauda Günther, 1878, loc. cit.; 1887, Rep. Sci. Res. Voy. Challenger, Zool., 22: 188, pl. 26, fig. B.

Bathypterois longipes Günther, 1887, Rep. Sci. Res. Voy. Challenger, Zool., 22: 188, pl. 48, fig. A; Goode and Bean, 1895, Ocean Ichth., p. 66, fig. 76; Jordan and Evermann, 1896, Bull. U. S. Nat. Mus., 47: 546; Boulenger, 1904, Cambr. Nat. Hist., 7: 613; Murray and Hjort, 1912, Depths of Ocean, pp. 396, 414, 416, 418; Koefoed, 1927, Rep. Sci. Res. M. Sars No. Atl. Deep-sea Exp. 1910, 4, (1), p. 63; Parr, 1928, Bull. Bingham 
Oceanogr. Coll., 3, (3), p. 26; Fowler, 1936, Bull. Amer. Mus. Nat. Hist., 70: 357 , fig. 170 .

Deep-abyssal records.--South Atlantic off Montevideo, 4846 meters, two specimens.

Western Atlantic, ca. $36^{\circ} \mathrm{N}$., $69^{\circ} \mathrm{W}$., 4593 meters, one specimen.

Eastern Atlantic, ca. $34^{\circ} \mathrm{N}$., $33^{\circ} \mathrm{W}$., 2620 meters, two specimens.

?South Pacific, ca. $39^{\circ}$ S., $131^{\circ}$ W., 4663 meters, one specimen.

Distribution.-Eastern Atlantic, one deep-abyssal record. Western Atlantic in the Gulf of Mexico and off the United States coast $\left(36^{\circ}-39^{\circ} \mathrm{N}\right.$.), one deep-abyssal specimen and three in 1747, 986 and 827 meters. South Atlantic off Montevideo, one deep-abyssal record. ?South Pacific, one deep-abyssal record.

Length. -63.5-229 mm.

Remarks. - B. longicauda was based on a young and damaged specimen; its identity with longipes is not certain.

\section{Bathypterois filiferus Gilchrist}

Bathypterois filiferus Gilchrist, 1906, Mar. Invest. So. Afr., 4: 166, pl. 48Atlantic off South Africa, 1645 meters.

Bathypterois filiferus Gilchrist and von Bonde, 1924, Rep. Fish. Mar. Biol. Surv. So. Afr., 3, (7), p. 7; Barnard, 1925, Ann. So. Afr. Mus., 21 : 233; Smith, 1949, Sea Fishes So. Afr., p. 114.

Bathypterois capensis Smith, 1949, op. cit., pl. 6, fig. 183.

Deep-abyssal records.-Off Cape Point, South Africa, ca. $33^{\circ}$ S., $16^{\circ}$ E., 2561, 2231 meters, five specimens in two hauls.

Distribution.-South Africa off Cape Point, five deep-abyssal specimens and twenty-two in 1098-1829 meters.

Length. - 223 (standard) $-300 \mathrm{~mm}$.

Remarks.-See remarks under B. capensis.

\section{Bathypterois insularum Alcock}

Bathypterois insularum Alcock, 1892, Ann. Mag. Nat. Hist., (6), 10: 356Arabian Sea, ca. $14^{\circ}$ N., $72^{\circ}$ E.

Bathypterois insularum Alcock, 1899, Descr. Cat. Indian Deep-sea Fishes, p. 158; Alcock and McArdle, 1900, Ill. Zool. Investigator, Fishes, pl. 32, fig. 1; Parr, 1928, Bull. Bingham Oceanogr. Coll., 3, (3), p. 27; Misra, 1950, Rec. Indian Mus., 45: 427; 1953, op. cit., 50: 416, fig. 26, $a$.

Deep-abyssal record and distribution.-Arabian Sea, 2084 meters, two specimens.

Length. $-140 \mathrm{~mm}$. 


\section{Bathypterois capensis Gilchrist and von Bonde}

Bathypterois capensis Gilchrist and von Bonde, 1924, Rep. Fish. Mar. Biol. Surv. So. Afr., 3, (7), p. 8, pl. 2, fig. 1-Atlantic off South Africa, ca. $33^{\circ} \mathrm{S} ., 16^{\circ} \mathrm{E}$.

Bathypterois Gilchrist, 1922, Rep. Fish. Mar. Biol. Surv. So. Afr., 2: 44.

Bathypterois capensis Barnard, 1925, Ann. So. Afr. Mus., 21: 233; Smith, 1949, Sea Fishes So. Afr., p. 114 (part, not pl. 6, fig. 183).

Deep-abyssal record and distribution.-Off Table Bay, South Africa, 2231 meters, three specimens.

Length.-(?)-200 $\mathrm{mm}$.

Remarks.-Parr $(1928$, p. 27) believed this species to be a synonym of $B$. insularum but it seems to differ from that species in having a deeper body and shorter tail. The range according to Smith (1949, p. 114) is "from the Cape to Algoa Bay in 800-1200 fathoms" but there is no information about the source of the added locality and depth (Algoa Bay, 800 fathoms). This locality may apply to the figured specimen (op. cit., pl. 6, fig. 183), which appears to belong to $B$. filiferus rather than to $B$. capensis as it is labeled, judging by the position of the anal fin, the scalation, and the length and form of the caudal peduncle.

\section{Family IPNOPIDAE}

A highly specialized and almost exclusively deep-abyssal family, which includes only five species.

\section{Bathymicrops regis Hjort and Koefoed}

Bathymicrops regis Hjort and Koefoed, in Murray and Hjort, 1912, Depths of Ocean, pp. $88,396,416,686,687$, figs. $305,501, d$-Atlantic, ca. $28^{\circ}$ N., $24^{\circ}$ W., 5000 meters.

Bathymicrops regis Koefoed, 1927, Rep. Sci. Res. M. Sars No. Atl. Deep-sea Exp. 1910, 4, (1), p. 64, pl. 5, figs. 1-3; Parr, 1928, Bull. Bingham Oceanographic Coll., 3, (3), p. 23; Fowler, 1936, Bull. Amer. Mus. Nat. Hist., 70: 347, fig. 163; Nybelin, 1951, Rep. Swedish Deep-sea Exp. 1947-1948, Zool., 2, (1), pp. 13, 16, 19; Marshall, 1954, Aspects Deep Sea Biol., p. 232 , fig. ix -7 .

Deep-abyssal records and distribution.-Eastern Atlantic, ca. $1^{\circ}$ N., $18^{\circ}$ W., 5250-5300 meters, four specimens.

Eastern Atlantic, 5000 meters, the type.

Eastern Atlantic, ca. $29^{\circ}$ N., $17^{\circ}$ W., 4267-4255 meters, two specimens.

Western Atlantic, ca. $12^{\circ}$ N., 52 ${ }^{\circ}$ W., 5044-5033 meters, one specimen. 
Apparently restricted to the abyssal plain.

Length.-?-110 $\mathrm{mm}$.

\section{Bathymicrops sewelli Norman}

Bathymicrops sewelli Norman, 1939, Sci. Rep. John Murray Exp. 1933-34, 7, (1), p. 26, fig. 7-Arabian Sea, ca. $9^{\circ}$ N., $55^{\circ} \mathrm{E}$.

Deep-abyssal record and distribution.-Arabian Sea, 3840-3872 meters, one specimen.

Length. $-350 \mathrm{~mm}$.

Remarks.-Harry (MS.) has remarked that the differences between this species and $B$. regis are such that $B$. sewelli should probably be placed in a separate genus.

\section{Ipnops murrayi Günther}

Ipnops murrayi Günther, 1878, Ann. Mag. Nat. Hist., (5), 2: 187-type locality not stated, here designated as Challenger Station 133, Atlantic near Tristan da Cunha, ca. $35^{\circ}$ S., $20^{\circ}$ W., 3475 meters, the locality of the specimen figured in Günther, 1887.

Ipnops murrayi Günther, 1887, Rep. Sci. Res. Voy. Challenger, Zool., 22: 191, pl. 49, fig. B; Moseley, 1887, op. cit., p. 269, pls. 67, 68; Goode and Bean, 1895, Ocean. Ichth., p. 67, figs. 67-68; Jordan and Evermann, 1896, Bull. U. S. Nat. Mus., 47: 547; 1900, op. cit., fig. 239; Boulenger, 1904, Cambr. Nat. Hist., 7: 613; Gilchrist, 1906, Mar. Invest. So. Afr., 4: 166; Murray and Hjort, 1912, Depths of Ocean, p. 686, fig. 501, e; Weber and de Beaufort, 1913, Fishes Indo-Austr. Arch., 2: 179, fig. 70; Barnard, 1925, Ann. So. Afr. Mus., 21 : 231; Parr, 1928, Bull. Bingham Oceanogr. Coll., 3, (3), p. 23; Norman, 1939, Sci. Rep. John Murray Exp. 1933-34, 7, (1), p. 27; Smith, 1949, Sea Fishes So. Afr., p. 117, fig. 189.

Deep-abyssal records.-Western Pacific, Celebes Sea, ca. $2^{\circ} \mathrm{N}$., $124^{\circ}$ E., 3932 meters, one specimen.

South Atlantic near Tristan da Cunha, 3475 meters, two specimens.

South Atlantic off Brazil, ca. $10^{\circ}$ S., $35^{\circ}$ W., 2926 meters, one specimen.

Western Atlantic off Bequia Island, 2756 meters, one specimen.

Western Atlantic, Gulf of Mexico, 2104-2194 meters, one specimen (Oregon Station 1303, $28^{\circ} 47^{\prime}$ N., $87^{\circ} 50^{\prime}$ W., May 26, 1955).

Distribution.-Off Cape Point, South Africa, one specimen in 1463-1645 meters. Western Atlantic, two deep-abyssal specimens and one in the Gulf of Mexico in 1747 meters. South Atlantic, two deep-abyssal records. Western Pacific, one deep-abyssal record. 
Indian Ocean near Zanzibar, three specimens in 1789 meters. Probably a deep-abyssal form, extending also to the abyssal plain.

Length. $-83-140 \mathrm{~mm}$.

\section{Ipnops agassizi Garman}

Ipnops Agassizii Garman, 1899, Mem. Mus. Comp. Zool., 24: 259, pl. H,

fig. 2-Pacific off the Galapagos Islands, ca. $2^{\circ} \mathrm{N} ., 96^{\circ} \mathrm{W}$.

Ipnops Agassizii Lendenfeld, 1905, op. cit., 30: 202, pl. 1, figs. 3-5.

?Ipnops Agassiz, 1906, op. cit., 33: 6.

Deep-abyssal record and distribution.-Eastern Pacific, 2488 meters, (?) specimens.

\section{Length. $-165 \mathrm{~mm}$.}

Remarks.-Agassiz (1906, p. 6) mentioned that this species was again caught in bottom hauls north of Callao, Peru, where dredging was carried on by the Albatross almost entirely below 3660 meters, the only exception being 838 meters, Station 4673 .

\section{Ipnops pristibrachium Fowler}

Ipnoceps pristibrachium Fowler, 1943, Bull. U. S. Nat. Mus., 100, (14), p. 56, fig.-Pacific, Gulf of Tomini, 1393 meters.

Deep-abyssal record.-Western Pacific, Gulf of Tomini, 1992 meters, one specimen.

Distribution.-Western Pacific, Gulf of Tomini, one deep-abyssal specimen and two in 1525 and 1393 meters.

Length.-128-158 mm.

\section{Family EURYPHARYNGIDAE}

\section{Eurypharynx pelecanoides Vaillant. Table 7.}

Eurypharynx pelecanoides Vaillant, *1882, C. R. Acad. Sci. Paris, 95: 1226 -Atlantic off Morocco (see footnote, p. 110).

Eurypharynx pelecanoides Vaillant, *1883, Nature, Paris, p. 131, fig.; 1883, Ann. Mag. Nat. Hist., (5), 11 : 67; 1888, Exp. Sci. Trav. Talis., Poiss., p. 198, pl. 17, fig. 1; Gill and Ryder, 1883, Proc. U. S. Nat. Mus., 6: 271; 1884, op. cit., 7: 48; Goode and Bean, 1895, Ocean. Ichth., p. 159, fig. 177; Jordan and Evermann, 1896, Bull. U. S. Nat. Mus., 47: 406; Roule, 1914, C. R. Acad. Sci. Paris, 158: 1822; 1916, Bull. Inst. Océanogr. Monaco, 320 : 2; 1919, Rés. Camp. Sci. Monaco, 52: 94; 1934, Poiss. Monde Viv. Eaux, 7 : 235, text fig. and p. 250, pl.; Norman, 1930, Disc. Rep., 2 : 337; Borodin, 1931, Bull. Mus. Comp. Zool., 72: 75; Roule and Angel, 1933, Rés. Camp. Sci. Monaco, 86: 73; Zugmayer, in Roule and Angel, 1933, op. cit., p. 85; Bertin, 1934, Bull. Mus. Hist. Nat. Paris, (2), 6: 30; 1934, 
Dana Rep., 3: 1, pl. 1, fig. 2, text figs. 1-26; 1938, op. cit., 15: 13; Parr, 1934, Bull. Bingham Oceanogr. Coll., 4, (6), p. 6; 1937, op. cit., 3, (7), p. 60; Fowler, 1936, Bull. Amer. Mus. Nat. Hist., 70: 327, fig. 154; Beebe, 1937, Zoologica, 22: 202; Schroeder, 1940, Copeia, p. 233; Tchernavin, 1946, Nature, London, 158: 667, fig.; 1947, Jour. Linn. Soc. London, 41 : 298 , pl. 2, fig. 1, pl. 3, figs. 1, 2, text figs. 2, 3, 6-11, 15, A, B; 1947, op. cit., p. 378, pl. 8, text figs. 1-8; Lozano Rey, 1947, Mem. R. Acad. Cien. Madrid, Ser. Cien. Nat., 11: 577, fig. 169; Nybelin, 1948, Göteborgs K. Vetensk. Vitt.-Samh. Handl., (B), 5, (16), p. 48; Marshall, 1954, Aspects Deep Sea Biol., pp. 141, 227, 240, pl. 4 and fig. ix-11; Koefoed, 1955, Rep. Sci. Res. M. Sars No. Atl. Deep-sea Exp. 1910, 4, (2), (4), p. 9, pl. 2, fig. D; Grey, 1955, Fieldiana, Zool., $37: 289$.

Saccopharynx flagellum Goode and Bean, 1883, Bull. Mus. Comp. Zool., $10: 223$.

Gastrostomus bairdii Gill and Ryder, 1883, Proc. U. S. Nat. Mus., 6: 271; 1884, op. cit., 7: 65; Gill, 1884, Nature, London, 29: 236; Goode and Bean, 1895, Ocean. Ichth., p. 159, figs. 180, 182; Jordan and Evermann, 1896, Bull. U. S. Nat. Mus., 47: 406; 1900, op. cit., fig. 176; Zugmayer, 1911, Rés. Camp. Sci. Monaco, 35 : 88, pl. 4, fig. 3; 1913, Bull. Inst. Océanogr. Monaco, 254: 1, fig.; Murray and Hjort, 1912, Depths of Ocean, pp. 97, $101,104,118,605,612,618,625,627,664,665,667,681,699,739-741$, 749 , 750, figs. $83, a, 459,490,529,530,545$; Pappenheim, 1914, Deutsche Südpolar-Exp. 1901-1903, 15, Zool., 7: 185; Nusbaum-Hilarowicz, 1915, Bull. Inst. Océanogr. Monaco, 307 : 1; 1915, op. cit., 313: 1; 1916, op. cit., 315: 1 ; 1923, Rés. Camp. Sci. Monaco, 65: 3, 10, 25, 52, pls. 1, 3, figs. 15-21, pls. 4, 5, figs. 1, 2, pl. 7, fig. 14, pls. 8-10, figs. 1-4; Hollister, 1930, Bull. N. Y. Zool. Soc., 33: 72, fig.; Beebe, 1930, op. cit., p. 57, fig.; 1934, Nat. Geogr. Mag., 66: 699, pl. 15; 1936, Bull. N. Y. Zool. Soc., 39: 105, pl.

Saccopharynx pelecanoides Günther, 1887, Rep. Sci. Res. Voy. Challenger, Zool., 22 : 262.

Saccopharynx bairdii Günther, 1887, op. cit., p. 262.

Megalopharynx longicaudatus Brauer, ${ }^{*} 1900$, Schild. deutschen Tiefsee Exp., p. 521.

Macropharynx longicaudatus Brauer, 1902, Zool. Anz., 25: 290; 1906, Wiss. Ergebn. Deutschen Tiefsee Exp. Valdivia, 15, (1), p. 134, pl. 8.

Gastrostomus pacificus Bean, 1904, Smiths. Misc. Coll., 45: 254, fig. 31.

?Eurypharynx sp. Agassiz, 1906, Mem. Mus. Comp. Zool., 33: 12.

Leptocephalus Gastrostomi Bairdii Lea, 1913, Rep. Sci. Res. M. Sars No. Atl. Deep-sea Exp. 1910, 3, (1), p. 35, pl. 6, fig. 5.

Eurypharynx (Gastrostomus) pacificus Roule, 1914, C. R. Acad. Sci. Paris, 158: 1822 .

?Eurypharynx richardi Roule, 1914, op. cit., p. 1823; 1916, Bull. Inst. Océanogr. Monaco, 320: 3; 1919, Rés. Camp. Sci. Monaco, 52: 94, pl. 6, fig. 1; Fowler, 1925, Amer. Mus. Nov., 162: 2; 1925, Copeia, p. 75; 1936, Bull. Amer. Mus. Nat. Hist., 70: 327, fig. 154; Bertin, 1934, Dana Rep., 3: 30; 1934, Bull. Mus. Hist. Nat. Paris, 6: 30; Parr, 1937, Bull. Bingham Oceanogr. Coll., 3, (7), p. 60; Tchernavin, 1947, Jour. Linn. Soc. London, 41 : 299; Belloc, 1949, Bull. Inst. Océanogr. Monaco, 958: 4. 
Eurypharynx Bertin, 1932, Bull. Soc. Zool. France, 57: 145, figs. 2, 4; Berg, 1948, C. R. Acad. Sci. Moscow, 59: 1353; Nybelin, 1951, Rep. Swedish Deep-sea Exp. 1947-1948, Zool., 2, (1), p. 20.

Gastrostomus Bertin, 1932, Bull. Soc. Zool. France, 57 : 148, figs. 5, 6; Gregory, 1933, Trans. Amer. Phil. Soc., 23 : 213, fig. 94.

?Eurypharynx sp. Tchernavin, 1947, Jour. Linn. Soc. London, 41 : 299, fig. 1.

Distribution.-Atlantic Ocean from ca. $50^{\circ}$ N., $27^{\circ} \mathrm{W}$. to ca. $7^{\circ} \mathrm{S} ., 8^{\circ} \mathrm{W}$. in the east, to ca. $17^{\circ} \mathrm{N}$. in the west and, centrally, to ca. $11^{\circ} \mathrm{S} ., 18^{\circ} \mathrm{W}$. (Table 7). Eastern Pacific between the Galapagos Islands and Peru, one specimen, 549 meters. Mid-Pacific between Midway and Guam, one specimen. ${ }^{1}$ Southwestern Pacific near New Zealand, ca. $41^{\circ}$ S., $176^{\circ}$ E. and ca. $33^{\circ}$ S., $165^{\circ}$ E., two specimens, 2000 and 2500 meters. Western Pacific around the Dutch East Indies, ca. $5^{\circ}$ S. $-6^{\circ}$ N., $123^{\circ}-138^{\circ}$ E., nine specimens, $2000-3500$ meters. North Indian Ocean, ca. $12^{\circ} \mathrm{S}$., $96^{\circ}$ E., one specimen, 1700 meters.

E. pelecanoides is known only from tropical and temperate seas, in deep water, and has been taken most frequently in the Atlantic. Its center of distribution seems to be in about $1400-2800$ meters, with perhaps a deeper area of concentration, in the eastern Atlantic, at 3400-4200 meters. There is one closing net record, in 1500 meters (Borodin, 1931). At least 250 specimens have been recorded and the species may therefore be considered a relatively common one. Extreme range 549-7625 meters.

Length. $-77-600 \mathrm{~mm}$.

\section{Family SIMENCHELYIDAE}

The family contains only one species.

\section{Simenchelys parasiticus Gill}

Simenchelys parasiticus Gill, in Goode and Bean, 1879, Bull. Essex Inst., 11 : 27-Atlantic off New England.

Simenchelys parasiticus Goode, 1881, Proc. U. S. Nat. Mus., 3: 485; Jordan and Gilbert, 1882, Bull. U. S. Nat. Mus., 16: 363; Günther, 1887, Rep. Sci. Res. Voy. Challenger, Zool., 22: 252; Gill, 1890, Proc. U. S. Nat. Mus., 13: 239; Jordan and Davis, 1891, Rep. U. S. Comm. Fish, 1888: 670; Goode and Bean, 1895, Ocean. Ichth., p. 139, fig. 161; Jordan and Evermann, 1896, Bull. U. S. Nat. Mus., 47: 349; 1900, op. cit., fig. 144;

${ }^{1}$ Bertin (1934, p. 28) listed the mid-Pacific specimen as having come from the surface, but it was probably caught in much deeper water. The fish was found entangled in the sounding wire over a bottom depth somewhere between 3660 and 5486 meters. 
Collett, 1896, Rés. Camp. Sci. Monaco, 10: 156, pl. 5, fig. 22, pl. 6, fig. 2; Albert I of Monaco, 1898, Geogr. Jour., 12: 461, fig.; Zugmayer, 1911, Rés. Camp. Sci. Monaco, 35: 83; Cockerell, 1913, Proc. Biol. Soc. Washington, 26: 75; Schmidt, *1913, Medd. Komm. Havunders., Ser. Fisk., 3, (1), p. 14, pl. 2; Vaillant, in Roule, 1919, Rés. Camp. Sci. Monaco, 52: 135; Jaquet, 1920, op. cit., 56:2, pls. 1-5; Bigelow and Welsh, 1925, Bull. U. S. Bur. Fish., 40, (1), p. 83, fig. 34 ; Barnard, 1925, Ann. So. Afr. Mus., 21 : 181, pl. 8, fig. 6; Tanaka, 1928, Fig. Descr. Fishes Japan, 42: 810, pl. 173, fig. 476; Roule, 1934, Poiss. Monde Viv. Eaux, 7 : 219-220, fig.; Fowler, 1936, Bull. Amer. Mus. Nat. Hist., 70 : 263, fig. 122; Lozano Rey, 1947, Mem. R. Acad. Cien. Madrid, Ser. Cien. Nat., 11: 514, fig. 164; Smith, 1949, Sea Fishes So. Afr., p. 387, fig. 1093; Bigelow and Schroeder, 1953, Fish. Bull., Fish Wildlife Serv., 53, (74), p. 157, fig. 71; Scholander and Van Dam, 1953, Biol. Bull., 104: 76.

Conchognathus grimaldii Collett, *1889, Bull. Soc. Zool. France, 14: 123.

Gymnosimenchelys leptosomus Tanaka, 1908, Jour. Coll. Sci. Imp. Univ. Tokyo, 23, (13), p. 2, pl. 2, fig. 2.

Simenchelys dofleini Franz, 1910, Abh. Bayer. Akad. Wiss., 4, Suppl., p. 10, pl. 3, figs. 1, 2.

Simenchelys leptosomus Jordan and Thompson, 1914, Mem. Carnegie Mus., 6: 233 .

Deep-abyssal records.-Eastern Atlantic, ca. $46^{\circ}$ N., $5^{\circ}$ W., 2620 meters, fourteen specimens.

Eastern Atlantic, ca. $32^{\circ} \mathrm{N}$., $17^{\circ} \mathrm{W}$., 2480 meters, two specimens.

Eastern Atlantic, ca. $38^{\circ} \mathrm{N}$., $30^{\circ} \mathrm{W}$., 2000 meters, two specimens.

Distribution.-Eastern Atlantic from ca. $15^{\circ}$ to $46^{\circ} \mathrm{N}$., $2^{\circ}-33^{\circ}$ W., three deep-abyssal records and many specimens in 880-1779 meters. Western Atlantic, $38^{\circ}-44^{\circ}$ N., $56^{\circ}-73^{\circ} \mathrm{W}$, common, 366-1645 meters. Off Cape Point, South Africa, (?) specimens, 1482 meters. Western Pacific, Japan, off Province Izu to Hokkaido, "rare" in ca. 731 meters. Probably not common in deep-abyssal waters. Center of distribution apparently between 700 and 1400 meters. Locally abundant.

Length. $-95-610 \mathrm{~mm}$.

\section{Family NETTASTOMIDAE}

A family of deep-sea eels with about seven genera and fifteen species, nettastomids are found in temperate and tropical seas throughout the world. Members of the genus Venefica seem to be inclined toward very deep water but there are only four deepabyssal records. The species are little known and, although they are perhaps benthic, cannot with certainty be included in the deep-abyssal fauna. 


\section{Venefica proboscidea Vaillant}

Nettastoma proboscideum Vaillant, 1888, Exp. Sci. Trav. Talis., Poiss., p. 84, pl. 7, fig. 3-Atlantic off Morocco, 2200 meters, Talisman Station 39 (see footnote, p. 110).

Venefica proboscidea Jordan and Davis, 1891, Rep. U. S. Comm. Fish, 1888: 652; Goode and Bean, 1895, Ocean. Ichth., p. 150; Lloyd, 1909, Mem. Indian Mus., 2: 151; Barnard, 1925, Ann. So. Afr. Mus., 21 : 194; Fowler, 1936, Bull. Amer. Mus. Nat. Hist., 70: 281; Norman, 1939, Sci. Rep. John Murray Exp. 1933-34, 7, (1), p. 37, fig. 10; Lozano Rey, 1947, Mem. R. Acad. Cien. Madrid, Ser. Cien. Nat., 11: 541, pl. 8, fig. 3; Smith, 1949, Sea Fishes So. Afr., p. 395.

Deep-abyssal records.-Eastern Atlantic off Morocco, 2200 meters, one specimen.

Indian Ocean, Arabian Sea, ca. $22^{\circ}$ N., $64^{\circ}$ E., 1893 meters, one specimen.

Distribution.-Eastern Atlantic, one deep-abyssal specimen. South Africa off Cape Point, one specimen, 1207 meters. Indian Ocean, one deep-abyssal record and, in the Gulf of Manaar, one specimen, 1624 meters.

Length.-510-960 mm.

\section{Venefica procera Goode and Bean}

Nettastoma procerum Goode and Bean, 1883, Bull. Mus. Comp. Zool., 10: $224-$ ca. $33^{\circ} \mathrm{N} ., 76^{\circ} \mathrm{W} ., 1183$ meters.

Nettastoma procerum Günther, 1887, Rep. Sci. Res. Voy. Challenger, Zool., 22: 253 .

Venefica procera Jordan and Davis, 1891, Rep. U. S. Comm. Fish, 1888 : 652; Goode and Bean, 1895, Ocean. Ichth., p. 149, fig. 168; Jordan and Evermann, 1896, Bull. U. S. Nat. Mus., 47: 365; Weber, 1913, Monogr. Siboga Exp., 57: 45, pl. 5, fig. 4; Weber and de Beaufort, 1916, Fishes Indo-Austr. Arch., 3: 271, figs. 118, 119.

Deep-abyssal record.-Western Atlantic, Gulf of Mexico, 21042194 meters, three specimens in one haul (Oregon Station 1303, $28^{\circ}$ $47^{\prime}$ N., $87^{\circ} 50^{\prime}$ W., May 26, 1955).

Distribution.-Western Atlantic, one deep-abyssal record and four specimens in 326-1183 meters from three hauls in the Caribbean Sea (ca. $16^{\circ}$ N., $62^{\circ}$ W.) and off the United States coast $\left(33^{\circ}-\right.$ $34^{\circ}$ N., $76^{\circ}$ W.). Western Pacific, Celebes Sea, one specimen in 1301 meters.

Length.-? and 520-ca. $800 \mathrm{~mm}$.

Remarks. - The statement by Jordan and Evermann (1896, p. 366) that the Albatross captured a specimen off San Pedro, 
California, is probably an error. The specimen referred to is perhaps $V$. tentaculata Garman, recorded by Gilbert in 1915 (p. 309).

Weber's statement $(1913$, p. 45$)$ that the types were taken in 1993 meters is also an error, and I find no record from 2200 meters, the lower limit of the range of the species according to Weber.

\section{Venefica ocella Garman}

Venefica ocella Garman, 1899, Mem. Mus. Comp. Zool., 24: 318, pl. 61, fig. 2-Pacific off Panama, ca. $5^{\circ} \mathrm{N} ., 86^{\circ} \mathrm{W}$.

Deep-abyssal record and distribution.-Eastern Pacific, 1951 meters, one specimen.

Length. $-940 \mathrm{~mm}$.

\section{Family CONGRIDAE}

Only one specimen of this large and well-known family of eels has been taken from deep-abyssal waters, although numerous deepsea species are found at lesser depths.

\section{Promyllantor purpureus Alcock}

Promyllantor purpureus Alcock, 1890, Ann. Mag. Nat. Hist., (6), 6: 310Arabian Sea, ca. $11^{\circ}$ N., $74^{\circ}$ E.

Promyllantor purpureus Alcock, 1892, Ill. Zool. Investigator, Fishes, pl. 6, fig. 2; 1899, Descr. Cat. Indian Deep-sea Fishes, p. 202; Goode and Bean, 1895, Ocean. Ichth., pp. 139, 517.

Deep-abyssal record and distribution.--Arabian Sea, 1829 meters, one specimen.

Length. $-432 \mathrm{~mm}$.

\section{Family ILYOPHIDAE}

\section{Ilyophis brunneus Gilbert}

Ilyophis brunneus Gilbert, in Jordan and Davis, 1891, Rep. U. S. Comm. Fish, 1888: 671 -Pacific near the Galapagos Islands, ca. $0^{\circ} 36^{\prime} \mathrm{S} ., 89^{\circ} \mathrm{W}$., 1159 meters.

Ilyophis brunneus Gilbert, 1891, Proc. U. S. Nat. Mus., 14: 352; Goode and Bean, 1895, Ocean. Ichth., p. 141, fig. 162; Jordan and Evermann, 1896, Bull. U. S. Nat. Mus., 47: 350; ?Lea, 1913, Rep. Sci. Res. M. Sars No. Atl. Deep-sea Exp. 1910, 3, (1), p. 18; Barnard, 1925, Ann. So. Afr. Mus., 21 : 182; Koefoed, 1927, Rep. Sci. Res. M. Sars No. Atl. Deep-sea Exp. 1910, 4, (1), p. 61; Parr, 1932, Bull. Bingham Oceanogr. Coll., 3, (5), p. 35, fig. 15; Smith, 1949, Sea Fishes So. Afr., p. 387, fig. 1092. 
?Leptocephalus Histiobranchi infernalis (or L. Ilyophidis brunnei) Lea, 1913, Rep. Sci. Res. M. Sars No. Atl. Deep-sea Exp. 1910, 3, (1), p. 14, pl. 2, fig. 5 .

Deep-abyssal record.-Eastern Atlantic, ca. $34^{\circ}$ N., $33^{\circ}$ W., 2615 meters, one specimen.

Distribution.-Eastern Atlantic, one specimen from deep-abyssal waters. Western Atlantic near the Bahama Islands, one specimen in 1298-1318 meters. Off Cape Point, South Africa, two specimens in 1463 meters. Eastern Pacific, one specimen in 1159 meters.

Length. $-120-575 \mathrm{~mm}$.

\section{Family SYNAPHOBRANCHIDAE}

Although pelagic when young, synaphobranch eels probably live on or near the ocean floor as adults, most of them in depths below 1000 meters. Only adult specimens are considered here. Five species have been found in deep-abyssal waters, one of them also known from the abyssal plain. S. infernalis, S. bathybius, and $S$. australis are closely related and may prove eventually to be forms of an almost exclusively deep-abyssal species.

The family is widely distributed in the Atlantic Ocean and is found also in the Indo-Pacific, near Hawaii, and in the north Pacific.

\section{Synaphobranchus infernalis Gill}

Histiobranchus infernalis Gill, 1883, Proc. U. S. Nat. Mus., 6: 255-Atlantic off U. S. coast, ca. $38^{\circ} \mathrm{N} ., 69^{\circ} \mathrm{W} ., 3166$ meters.

Synaphobranchus infernalis Günther, 1887, Rep. Sci. Res. Voy. Challenger, Zool., 22: 254; Regan, 1913, Trans. Roy. Soc. Edinburgh, 40: 235; Bruun, 1936, Bull. Inst. Océanogr. Monaco, 700 : 5; 1937, Dana Rep., 9: 5, figs. 1, 3, 10-12, 14, 17; Norman and Trewavas, 1939, Ann. Mag. Nat. Hist., (11), $3: 358$.

Histiobranchus infernalis Jordan and Davis, 1891, Rep. U. S. Comm. Fish, 1888: 673; Goode and Bean, 1895, Ocean. Ichth., p. 145, fig. 165; Jordan and Evermann, 1896, Bull. U. S. Nat. Mus., 47: 352; 1900, op. cit., fig. 147; Zugmayer, in Roule and Angel, 1933, Rés. Camp. Sci. Monaco, 86: 85; Fowler, 1936, Bull. Amer. Mus. Nat. Hist., 70: 1209; Jensen, 1948, Skr. Univ. Zool. Mus. København, 9: 59.

Histiobranchus bathybius or H. infernalis Lütken, 1898, Danish Ingolf Exp., 2, (1), p. 7.

?Leptocephalus Histiobranchi infernalis (or L. Ilyophidis brunnei) Lea, 1913, Rep. Sci. Res. M. Sars No. Atl. Deep-sea Exp. 1910, 3, (1), p. 14, pl. 2, fig. 5 .

Histiobranchus bathybius Koefoed, 1927, op. cit., 4, (1), p. 60. 
Leptocephalus Synaphobranchus infernalis Bruun, 1937, Dana Rep., 9: 11, pl. 1 , fig. 5 .

Deep-abyssal records.-Western Atlantic, 3166 meters, the type.

Davis Strait, ca. $60^{\circ} \mathrm{N}$., $54^{\circ} \mathrm{W}$., 3136 meters, one specimen.

Davis Strait, ca. $61^{\circ} \mathrm{N}$., $56^{\circ} \mathrm{W} ., 2624$ meters, one specimen.

Western Atlantic, ca. $36^{\circ} \mathrm{N}$., $74^{\circ} \mathrm{W}$., 2266 meters, one specimen.

Mid-Atlantic on the mid-Atlantic ridge, ca. $45^{\circ} \mathrm{N} ., 25^{\circ}$ W., 3120 meters, two specimens.

Eastern Atlantic, ca. $34^{\circ} \mathrm{N}$., $33^{\circ} \mathrm{W}$., 2615 meters, four specimens.

Distribution.-Eastern Atlantic, one deep-abyssal record, one specimen in 1560 meters (ca. $37^{\circ} \mathrm{N}$., $25^{\circ} \mathrm{W}$.) and one young example caught in mid-water in $1500-2000$ meters, a doubtful identification (ca. $48^{\circ} \mathrm{N} ., 13^{\circ}$ W.). Mid-Atlantic, one deep-abyssal record. Western Atlantic, Davis Strait and $31^{\circ}-38^{\circ} \mathrm{N}$., four deep-abyssal records and one specimen in 644 meters. Thirteen specimens in all, ten of them deep-abyssal.

Length. $-78-900 \mathrm{~mm}$. Leptocephali $30-90 \mathrm{~mm}$.

\section{Synaphobranchus bathybius Günther}

Synaphobranchus bathybius Günther, 1877, Ann. Mag. Nat. Hist., (4), 20 : 445 (part)-Pacific, ca. $36^{\circ}$ N., $178^{\circ}$ E., 3749 meters.

Synaphobranchus bathybius Günther, 1887, Rep. Sci. Res. Voy. Challenger, Zool., 22: 254, pl. 62, fig. B; Bruun, 1937, Dana Rep., 9: 7, fig. 2 ; Norman and Trewavas, 1939, Ann. Mag. Nat. Hist., (11), 3: 358.

Histiobranchus bathybius Jordan and Davis, 1891, Rep. U. S. Comm. Fish, 1888: 673; Gilbert, 1896, op. cit., 1893: 402; Jordan and Evermann, 1896, Bull. U. S. Nat. Mus., 47 : 352; Regan, 1913, Trans. Roy. Soc. Edinburgh, 40 : 235; Smith, 1949, Sea Fishes So. Afr., p. 386, fig. 1090 (part).

Synaphobranchus (Histiobranchus) bathybius Norman, 1939, Sci. Rep. John Murray Exp. 1933-34, 7, (1), p. 45.

Deep-abyssal records.--Mid-Pacific, 3749 meters, the type.

Western Pacific, ca. $34^{\circ}$ N., $140^{\circ}$ E., 3429 meters, nine specimens.

North Pacific, ca. $56^{\circ}$ N., $172^{\circ}$ W., 2971 meters, one specimen.

North Indian Ocean near Zanzibar, 2926 meters, one specimen.

Distribution.-Pacific in Bering Sea, off Japan and northwest of the Hawaiian Islands. North Indian Ocean. Exclusively deepabyssal and reaching the abyssal plain.

Length. $-258-622 \mathrm{~mm}$.

\section{Synaphobranchus australis Regan}

Synaphobranchus australis Regan, 1913, Trans. Roy. Soc. Edinburgh, 40: 235, pl. 8, fig. 5-Indian Ocean, ca. $46^{\circ} \mathrm{S}$., $45^{\circ}$ E., 2515 meters. 
Synaphobranchus bathybius Günther, 1877, Ann. Mag. Nat. Hist., (4), 20 : 445 (part); 1887, Rep. Sci. Res. Voy. Challenger, Zool., 22: 254 (part).

Synaphobranchus australis Regan and Trewavas, 1939, Ann. Mag. Nat. Hist., (11), $3: 359$.

Histiobranchus bathybius Smith, 1949, Sea Fishes So. Afr., p. 386 (part).

Deep-abyssal records and distribution.- South Atlantic, ca. $48^{\circ}$ S., $10^{\circ} \mathrm{W}$., 3185 meters, one specimen.

South Indian Ocean, 2515 meters, the type.

Length. $-350-470 \mathrm{~mm}$.

\section{Synaphobranchus kaupi Johnson. Table 8.}

Synaphobranchus kaupii Johnson, 1862, Proc. Zool. Soc. London, p. 169 Atlantic off Madeira.

Synaphobranchus pinnatus Günther, 1870, Cat. Fishes Brit. Mus., 8: 23; 1887, Rep. Sci. Res. Voy. Challenger, Zool., 22: 254, pl. 62, fig. A; Goode and Bean, 1879, Bull. Essex Inst., 11: 26; 1883, Bull. Mus. Comp. Zool., 10 : 223 ; 1895, Ocean. Ichth., p. 143, fig. 164; Goode, 1881, Proc. U. S. Nat. Mus., 3: 485; Vaillant, 1888, Exp. Sci. Trav. Talis., Poiss., p. 88, pl. 6, fig. 2; Vaillant, in Roule, 1919, Rés. Camp. Sci. Monaco, 52: 135; Jordan and Evermann, 1896, Bull. U. S. Nat. Mus., 47: 351; 1900, op. cit., fig. 146; Collett, 1896, Rés. Camp. Sci. Monaco, 10 : 54; 1905, Rep. Norwegian Fish. Mar. Invest., 2, (3), p. 45; Ehrenbaum, 1902, Fauna Arct., 2: 139; 1909, Nord. Plankton, Zool., 1 : 387, fig. 147; Bragança, *1903, Cat. Coll. Exp. Palacio Cristal Portuense, p. 40; Holt and Byrne, 1906, Rep. Fish. Ireland 1905, Sci. Invest., 2: 7, fig.; Schmidt, *1906, Rapp. Proc. Verb. Cons. Perm. Intern. Explor. Mer, 5: 236, pl. 9, figs. 4-6; ${ }^{*} 1909$, Medd. Komm. Havunders. Ser. Fisk., 3: 7 ; Byrne, 1907, Jour. Mar. Biol. Assoc., 8: 1; Zugmayer, 1911, Rés. Camp. Sci. Monaco, 35: 84; Zugmayer, in Roule and Angel, 1933, op. cit., 86: 85; Murray and Hjort, 1912, Depths of Ocean, pp. 80, 101, 395, 423, figs. 57, a, 264; Roule, 1919, Rés. Camp. Sci. Monaco, 52: 99, pl. 6, fig. 3, b; Barnard, 1925, Ann. So. Afr. Mus., 21 : 183; Bigelow and Welsh, 1925, Bull. U. S. Bur. Fish., 40, (1), p. 84, fig. 35; Koefoed, 1927, Rep. Sci. Res. M. Sars No. Atl. Deep-sea Exp. 1910 , 4, (1), p. 58, pl. 2, fig. 2; Parr, 1932, Bull. Bingham Oceanogr. Coll., 3, (5), p. 37; Noronha and Sarmento, *1934, Peixes Madeira, p. 166; Nobre, 1935, Fauna Marinha Portugal, 1: 390; Fowler, 1936, Bull. Amer. Mus. Nat. Hist., 70 : 265, fig. 123; Bruun, 1936, Bull. Inst. Océanogr. Monaco, 700: 5; 1937, Dana Rep., 9: 4; Coelho, 1942, Trav. Stat. Biol. Marit. Lisbonne, 46: 8; Lozano Rey, 1947, Mem. R. Acad. Cien. Madrid, Ser. Cien. Nat., 11 : 517, figs. 165, 166; Smith, 1949, Sea Fishes So. Afr., p. 386, fig. 1089; Bigelow and Schroeder, 1953, Fish. Bull., Fish Wildlife Serv., 53, (74), p. 158, fig. 72; Scholander and Van Dam, 1953, Biol. Bull., 104: 76; 1954, op. cit., 107: 249; Poll, 1953, Rés. Sci. Exp. Océanogr. Belge (1948-1949), 4, (2), (3), p. 154, fig. 63; Tucker, 1954, Bull. Brit. Mus. (Nat. Hist.), Zool., 2: 192; Marshall, 1954, Aspects Deep Sea Biol., pl. 3 . 
Nettophichthys retropinnatus Holt, *1891, Sci. Proc. Roy. Dublin Soc., 7 : 122; Holt and Calderwood, 1895, Trans. Roy. Dublin Soc., 5: 511, pl. 41, fig. 2.

Leptocephalus Synaphobranchi pinnati Murray and Hjort, 1912, Depths of Ocean, p. 751, fig. 549; Lea, 1913, Rep. Sci. Res. M. Sars No. Atl. Deepsea Exp. 1910, 3, (1), p. 12, pl. 2, figs. 1-4, text figs. 5-9; Barnard, 1925, Ann. So. Afr. Mus., 21: 218; Nybelin, 1948, Göteborgs K. Vetensk. Vitt.-Samh. Handl., (B), 5, (16), p. 54.

Synaphobranchus pinnatus var. parvipinnis Roule, 1916, Bull. Inst. Océanogr. Monaco, 320: 4; 1919, Rés. Camp. Sci. Monaco, 52: 99, pl. 6, fig. 3; Belloc, 1949, Bull. Inst. Océanogr. Monaco, 958: 3.

Synaphobranchus kaupi Bruun, 1937, Dana Rep., 9: 4, figs. 1, B, 2, 3, 5-9, 14, 17; Beebe, 1937, Zoologica, 22: 202; Norman and Trewavas, 1939, Ann. Mag. Nat. Hist., (11), 3: 355; Longley and Hildebrand, 1941, Pap. Tortugas Lab., 34: 16; Nybelin, 1948, Göteborgs K. Vetensk. Vitt.-Samh. Handl., (B), 5, (16), p. 52; Jensen, 1948, Skr. Univ. Zool. Mus. København, 9: 58 , chart 4 .

Deep-abyssal records.--?Mid-Atlantic, ca. $31^{\circ}$ N., $42^{\circ}$ W., 3465 meters, one specimen. ${ }^{1}$

Eastern Atlantic off the Cape Verde Islands, 3200 meters, two specimens.

Eastern Atlantic off the Azores, 2235, 2220 meters, two specimens.

Eastern Atlantic off Morocco, 2210, 2200 meters, twelve specimens in two hauls.

Eastern Atlantic, ca. $35^{\circ}$ N., $8^{\circ}$ W., 2150-2300 meters, twentyfour specimens in one haul.

Eastern Atlantic south of Iceland, ca. $62^{\circ}$ N., $19^{\circ}$ W., 2150 meters, one specimen.

Eastern Atlantic off Morocco and the Canary Islands, 20152115 meters, fifteen specimens in five hauls, one to eight per haul.

Eastern Atlantic, ca. $38^{\circ} \mathrm{N}$., 25 $5^{\circ} \mathrm{W}$., 1998 meters, one specimen.

Eastern Atlantic, ca. $57^{\circ}$ N., $11^{\circ}$ W., 1853 meters, three specimens.

Western Atlantic off United States coast, $39^{\circ}-40^{\circ}$ N., 2654, 2258, 2250, 2102, 2084, 1997 meters, an unstated number of specimens from six hauls.

Western Atlantic, Gulf of Mexico, 2104-2194 meters, four specimens (Oregon Station 1303, $28^{\circ} 47^{\prime}$ N., $87^{\circ} 50^{\prime}$ W., May 26, 1955).

1 This record (Zugmayer, 1911, p. 84) from Monaco Station 2108 may be an error. Neither Prince Albert I (1932, p. 40) nor Richard $(1934$, p. 333) mentioned the species in their notes on the catch made at that station. 
Distribution.-Atlantic from Davis Strait (ca. $66^{\circ}$ N.) to Brazil in the west and from ca. $62^{\circ} \mathrm{N} ., 19^{\circ} \mathrm{W}$. to ca. $5^{\circ} \mathrm{S} ., 11^{\circ} \mathrm{E}$. in the east; many specimens reported, more than seventy-one from deepabyssal waters, others in 236-1805 meters. South Africa off Cape Point, Agulhas Bank and East London, an unstated number of specimens, 732-1025 meters. Reaching deep-abyssal waters but with the center of distribution probably between 800 and 2000 meters.

Length. - 115-813 mm. Leptocephali 20-131 mm.

Remarks.-The specimens considered here are all from the Atlantic and off South Africa. Of the several Indo-Pacific forms that have been considered synonyms of $S$. kaupi by some authors, only $S$. brevidorsalis is known from deep-abyssal waters.

The example recorded by Günther (1887, p. 254) from 1200 fathoms off the coast of Brazil was actually caught at only about 700 fathoms, or 1280 meters (Thomson and Murray, 1895, p. 368).

\section{Synaphobranchus brevidorsalis Günther}

Synaphobranchus brevidorsalis Günther, 1887, Rep. Sci. Res. Voy. Challenger, Zool., 22: 255 (part), pl. 63, fig. C-Pacific north of New Guinea, ca. $1^{\circ} \mathrm{S} ., 144^{\circ}$ E., 1956 meters.

Synaphobranchus brevidorsalis Brauer, 1906, Wiss. Ergebn. Deutschen Tiefsee Exp. Valdivia, 15, (1), p. 134; 1908, op. cit., 15, (2), p. 160; Weber, 1913, Monogr. Siboga Exp., 57: 55; Weber and de Beaufort, 1916, Fishes Indo-Austr. Arch., 3: 335, fig. 163; Norman and Trewavas, 1939, Ann. Mag. Nat. Hist., (11), 3: 358; Matsubara and Ochiai, 1951, Jap. Jour. Ichth., 1 : 251, fig. 4, $c$.

Synaphobranchus pinnatus var. brevidorsalis Lloyd, 1909, Mem. Indian Mus., 2: 152, pl. 47.

Synaphobranchus (Synaphobranchus) brevidorsalis Norman, 1939, Sci. Rep. John Murray Exp. 1933-34, 7, (1), p. 45.

Deep-abyssal records.-Western Pacific north of New Guinea, 1956 meters, the type.

Western Pacific, ca. $2^{\circ}$ S., $130^{\circ}$ E., 1914 meters, two specimens.

Distribution.-Western Pacific, two deep-abyssal records, one specimen from the Banda or Celebes Sea, depth data lost, and one from Japan, depth unknown. Indian Ocean in the Arabian Sea, near the Maldive Islands and near Zanzibar, three specimens in 1789 meters, one in 1534 meters, one in $914-1463$ meters, and three in 693 meters.

Length. $-155-705 \mathrm{~mm}$.

Remarks. - The species is believed by some authors to be a form of S. kaupi. 


\section{Family SERRIVOMERIDAE}

Serrivomerids, a family of little-known bathypelagic eels, are found on both sides of the northern Atlantic, in the eastern and central Pacific, and in the Indian Ocean. It would not be surprising if some of them should eventually prove to belong to the deepabyssal fauna.

\section{Serrivomer parabeani Bertin. Table 9 .}

Serrivomer parabeani Bertin, 1940, C. R. Acad. Sci. Paris, 211 : 76-Atlantic, type locality not stated, here designated as Monaco Station 3281 , ca. $39^{\circ}$ N., $35^{\circ} \mathrm{W}$., locality of specimen described and figured by Roule and Angel, 1933, p. 71, pl. 4, fig. 33 (Paraserrivomer hasta, not of Zugmayer).

Serrivomer Murray and Hjort, 1912, Depths of Ocean, pp. 85, 93, 108, 755.

Serrivomer sector Murray and Hjort, 1912, op. cit., pp. 605, 612, 630.

Serrivomer sector longidentatus Roule and Bertin, 1929, Oceanogr. Rep. Danish Dana Exp. 1920-22, 4: 59 (part).

Paraserrivomer hasta Roule and Angel, 1931, Bull. Inst. Océanogr. Monaco, 581 : 3; 1933, Rés. Camp. Sci. Monaco, 86: 70, pl. 4, fig. 4; Fowler, 1936, Bull. Amer. Mus. Nat. Hist., 70: 1210 (part); Belloc, 1949, Bull. Inst. Océanogr. Monaco, 958: 4; ?Brandes, Kotthaus and Krefft, 1954, Cons.

Perm. Intern. Explor. Mer, Ann. Biol., 9: 48.

Nettastoma hasta Fowler, 1936, Bull. Amer. Mus. Nat. Hist., 70: 281 (part).

Serrivomer beani Beebe and Crane, 1936, Zoologica, 20: 61 (part).

Serrivomer hasta Norman, 1939, Sci. Rep. John Murray Exp. 1933-34, 7,

(1), p. 33; Lozano Rey, 1947, Mem. R. Acad. Cien. Madrid, Ser. Cien. Nat., 11 : 550, pl. 9, fig. 2.

Serrivomer parabeani Bertin, 1944, Bull. Mus. Hist. Nat. Paris, 16: 106, fig. 2, c; Bauchot-Boutin, 1953, op. cit., 25: 366; 1954, op. cit., 26: 304; Bauchot-Boutin, in Koefoed, 1955, Rep. Sci. Res. M. Sars No. Atl. Deepsea Exp. 1910, 4, (2), (4), p. 11.

Distribution.-Eastern Atlantic, ? ca. $63^{\circ} \mathrm{N} ., 11^{\circ} \mathrm{W}$. and in $28^{\circ}-57^{\circ} \mathrm{N}$. Mid-Atlantic, $31^{\circ}-40^{\circ} \mathrm{N}$. Western Atlantic off Bermuda and in $40^{\circ}-43^{\circ} \mathrm{N}$. Perhaps chiefly deep-abyssal. Extreme depth range $300-4500$ meters.

Length.-115-695 mm.

Remarks.-In a key to the species of Serrivomer based on Dana material, Bauchot-Boutin (1953, p. 366) included the Pacific and Indian oceans in the range of this species.

\section{Serrivomer sector Garman. Table 10.}

Serrivomer sector Garman, 1899, Mem. Mus. Comp. Zool., 24: 320, pl. 63one station in the Gulf of California in 1280 meters and nine in the Gulf of Panama (ca. $3^{\circ}$ N., $82^{\circ}$ W. to ca. $7^{\circ}$ N., $79^{\circ}$ W.) in 245,1409 and 1865-3240 meters (type locality not designated). 
Serrivomer beanii Gilbert, 1905, Bull. U. S. Fish Comm., 23: 586.

Serrivomer sector Brauer, 1906, Wiss. Ergebn. Deutschen Tiefsee Exp. Valdivia, 15, (1), p. 132, pl. 8; Lloyd, 1909, Mem. Indian Mus., 2: 152; Weber and de Beaufort, 1916, Fishes Indo-Austr. Arch., 3: 332, figs. 159, 160; Townsend and Nichols, 1925, Bull. Amer. Mus. Nat. Hist., 52: 12; Beebe and Crane, 1936, Zoologica, $20: 36$; Bertin, 1944, Bull. Mus. Hist. Nat. Paris, 16: 106; Bauchot-Boutin, 1953, op. cit., 25: 366, fig. 1; 1954, op. cit., 26: 303,305 .

Serrivomer sector longidentatus Roule and Bertin, 1929, Oceanogr. Rep. Danish Dana Exp. 1920-22, 4: 40 (part).

?Serrivomer microps Norman, 1939, Sci. Rep. John Murray Exp. 1933-34, $7,(1)$, p. 33 .

Distribution.-Indian Ocean from the Arabian Sea and off Sumatra (ca. $0^{\circ} 58^{\prime} \mathrm{S}$., $99^{\circ}$ E.) to ca. $26^{\circ}$ S., $93^{\circ}$ E. in the east and ca. $4^{\circ} \mathrm{N} ., 48^{\circ} \mathrm{E}$. in the west. Mid-Pacific off the Hawaiian Islands. Eastern Pacific in the Gulf of Panama, the Gulf of California, and off Lower California. Too few specimens to determine the center of distribution, perhaps in 1400-2400 meters (Table 10). Extreme depth range $245-3240$ meters.

\section{Length. $-82-580 \mathrm{~mm}$.}

Remarks.-Norman $(1939$, p. 33$)$ included this species in the synonymy of Serrivomer microps Alcock. In three brief papers, preliminary to a revision of the genus Serrivomer, neither Bertin (1944, p. 106) nor Bauchot-Boutin (1953, p. 366 and 1954, p. 305) included microps in their keys to the genus. The species microps was described as Gavialiceps microps by Alcock (1889, p. 461; 1892, p. $364 ; 1899$, p. 191), who reported it from three hauls in the Bay of Bengal and the Arabian Sea in 1588-1670, 1650 and 1911 meters. Under the name Serrivomer microps, Norman (1939, p. 33) recorded one specimen from the Laccadive Sea in 2505 meters, one from near Zanzibar in 1207-1463 meters, and one from an unknown depth near the Maldive Islands.

\section{Family NEMICHTHYIDAE}

Several species of this widely distributed bathypelagic family, especially those of the genus Avocettina, have been recorded from deep-abyssal waters, but such records are not frequent enough to warrant placing the habitat at these depths, with the possible exception of Avocettina infans Günther.

Avocettina infans Günther. Table 11.

Nemichthys infans Günther, 1878, Ann. Mag. Nat. Hist., (5), 2:251-Atlantic, ca. $5^{\circ}$ N., $14^{\circ}$ W., 4571 meters. 
Labichthys elongatus Gill and Ryder, 1883, Proc. U. S. Nat. Mus., 6: 262; Günther, 1887, Rep. Sci. Res. Voy. Challenger, Zool., 22: 263; Jordan and Davis, 1891, Rep. U. S. Comm. Fish, 1888: 656; Goode and Bean, 1895, Ocean. Ichth., p. 153, fig. 172; Jordan and Evermann, 1896, Bull. U. S. Nat. Mus., 47: 369.

Nemichthys infans Günther, 1887, Rep. Sci. Res. Voy. Challenger, Zool., 22: 264 , pl. 63, fig. B.

Avocettina infans Jordan and Davis, 1891, Rep. U. S. Comm. Fish, 1888: 655; Jordan and Evermann, 1896, Bull. U. S. Nat. Mus., 47: 367; Brauer, 1906, Wiss. Ergebn. Deutschen Tiefsee Exp. Valdivia, 15, (1), p. 129, pl. 8, figs. 5, 6; 1908, op. cit., 15, (2), p. 171; Weber, 1913, Monogr. Siboga Exp., 57: 53; Weber and de Beaufort, 1916, Fishes Indo-Austr. Arch., 3: 334; Roule and Bertin, 1924, Bull. Mus. Hist. Nat. Paris, 30: 63; 1929, Oceanogr. Rep. Danish Dana Exp. 1920-22, 4: 7, 23, pl. 1, fig. 2, text figs. 10-12 (part); 1931, Faune Ichth. Atl. Nord, 8, fig.; Barnard, 1925, Ann. So. Afr. Mus., 21 : 199; Forrest, ${ }^{*} 1927$, N. West. Nat. Arbroath, 2: 248, fig.; Norman, 1930, Disc. Rep., 2: 338; Roule and Angel, 1931, Bull. Inst. Océanogr. Monaco, 581 : 3; 1933, Rés. Camp. Sci. Monaco, 86: 69; ?Parr, 1932, Bull. Bingham Oceanogr. Coll., 3, (5), p. 15, fig. 7; Beebe, 1936, Zoologica, 21 : 201; ?1937, op. cit., 22 : 202; Beebe and Crane, 1936, op. cit., 20:61; 1937, op. cit., 22: 367; Fowler, 1936, Bull. Amer. Mus. Nat. Hist., 70 : 283, 1211, fig. 133; 1942, Arq. Zool. São Paulo, 3: 136; Chapman, 1940, Occ. Pap. British Columbia Prov. Mus., 2: 14; Smith, 1949, Sea Fishes So. Afr., p. 391; Koumans, 1953, Temminckia, 9: 196 .

Labichthys infans Goode and Bean, 1895, Ocean. Ichth., p. 153, figs. 173, 174. Avocettina elongata Jordan and Evermann, 1898, Bull. U. S. Nat. Mus., 47 : 2802.

Gavialiceps tinrayei Zugmayer, 1914, Bull. Inst. Océanogr. Monaco, 288: 3; Roule and Bertin, 1929, Oceanogr. Rep. Danish Dana Exp. 1920-22, 4: 59; Belloc, 1949, Bull. Inst. Océanogr. Monaco, 958: 4.

?Avocettina sp. Beebe and Crane, 1937, Zoologica, 23: 369, figs. 10-16.

Distribution.-Eastern Atlantic off Wales and from ca. $34^{\circ}$ to $0^{\circ} 26^{\prime} \mathrm{N}$. Mid-Atlantic, ca. $31^{\circ} \mathrm{N} .41^{\circ} \mathrm{W}$. Western Atlantic from $39^{\circ}$ N. to $8^{\circ} \mathrm{S}$. South Africa off Cape Point. South Atlantic, three localities: ca. $34^{\circ}$ S., $16^{\circ} \mathrm{W} . ; 39^{\circ}$ S., $36^{\circ} \mathrm{W}$.; and $41^{\circ}$ S., $42^{\circ} \mathrm{W}$. North Indian Ocean, $0^{\circ} 39^{\prime}$ to $13^{\circ} \mathrm{S} ., 41^{\circ}$ to $98^{\circ} \mathrm{E}$. Western Pacific around the Dutch East Indies. Number of specimens insufficient to determine the center of distribution, perhaps 1400-2600 meters. Extreme depth range 600-4571 meters.

Length. - 140-604 mm.

Remarks.-Although $A$. infans is a bathypelagic fish, Günther (1887, p. 264) reported a specimen found attached to a cable brought up from 1489 meters. 


\section{Family CYEMIDAE}

\section{Cyema atrum Günther. Table 12.}

Cyema atrum Günther, 1878, Ann. Mag. Nat. Hist., (5), 2: 251-Challenger Stations 158 (ca. $50^{\circ}$ S., $123^{\circ}$ E.) and 295 (ca. $38^{\circ}$ S., $94^{\circ}$ W.), 3291 and 2744 meters (type locality not designated).

Cyema atrum Günther, 1887, Rep. Sci. Res. Voy. Challenger, Zool., 22: 265, pl. 54, fig. D; Vaillant, 1888, Exp. Sci. Trav. Talis., Poiss., p. 91, pl. 7, fig. 4; Vaillant, in Roule, 1919, Rés. Camp. Sci. Monaco, 52: 135; Goode and Bean, 1895, Ocean. Ichth., p. 154, fig. 176; Brauer, 1906, Wiss. Ergebn. Deutschen Tiefsee Exp. Valdivia, 15, (1), p. 131, pl. 8, fig. 3; Zugmayer, 1911, Rés. Camp. Sci. Monaco, 35: 85, pl. 4, fig. 2; Zugmayer, in Roule and Angel, 1933, op. cit., 86: 85; Murray and Hjort, 1912, Depths of Ocean, pp. 87, 605, 612, 665, figs. 69, 460, 490; NusbaumHilarowicz, 1923, Rés. Camp. Sci. Monaco, 65: 75, pl. 3, fig. 11, pl. 10, figs. 5-11; Roule and Angel, 1924, Bull. Inst. Océanogr. Monaco, 451: 7; 1930, Rés. Camp. Sci. Monaco, 79: 115, pl. 6, fig. 151; Roule and Bertin, 1924, Bull. Mus. Hist. Nat. Paris, $30: 65 ;{ }^{*} 1929$, C. R. Soc. Biol. Paris, C, (4), p. 264; 1929, Oceanogr. Rep. Danish Dana Exp. 1920-22, 4: 52, 101, pl. 9, figs. 1-10, text figs. 35, 36, 55-77; Townsend and Nichols, 1925, Bull. Amer. Mus. Nat. Hist., 52: 12; Trewavas, 1933, Proc. Zool. Soc. London, p. 601, pl. 1, text figs. 1-3; Beebe, 1934, Nat. Geogr. Mag., 66: 698, pl. 14; 1937, Zoologica, 22: 202; Fowler, 1936, Bull. Amer. Mus. Nat. Hist., 70: 287, figs. 136, 137; Bertin, 1936, Bull. Soc. Zool. France, 61 : 440, figs. 1-5; 1937, Dana Rep., 10 : 3, figs. 1-24; Roule, 1937, Bull. Inst. Océanogr. Monaco, 726: 7; Tchernavin, 1947, Jour. Linn. Soc. London, 41 : 345; Lozano Rey, 1947, Mem. R. Acad. Cien. Madrid, Ser. Cien. Nat., 11 : 553, pl. 9, fig. 3; Nybelin, 1948, Göteborgs K. Vetensk. Vitt.-Samh. Handl., (B), 5, (16), p. 50; Marshall, 1954, Aspects Deep Sea Biol., p. 225, fig. ix-5; Koefoed, 1955, Rep. Sci. Res. M. Sars No. Atl. Deep-sea Exp. 1910, 4, (2), (4), p. 10; Grey, 1955, Fieldiana, Zool., $37: 291$.

Leptocephalus sp. Murray and Hjort, 1912, Depths of Ocean, p. 93, fig. 79.

Leptocephalus Cyematis atri Lea, 1913, Rep. Sci. Res. M. Sars No. Atl. Deepsea Exp. 1910, 3, (1), p. 16, pl. 2, fig. 6, text fig. 11.

Leptocephalus $F$ Roule, 1914, Bull. Inst. Océanogr. Monaco, 292: 9, figs. 5, 6; 1919, Rés. Camp. Sci. Monaco, 52: 105, pl. 7, fig. 6.

Cyema Hollister, 1930, Bull. N. Y. Zool. Soc., 33: 73, fig.

Distribution.-Eastern Atlantic from $37^{\circ}$ to $28^{\circ} \mathrm{N}$. and off the African coast in $23^{\circ}$ and $30^{\circ} \mathrm{S}$. Mid-Atlantic from ca. $39^{\circ} \mathrm{N}$., $39^{\circ} \mathrm{W}$. to ca. $7^{\circ} \mathrm{S}$., $8^{\circ} \mathrm{W}$. Western Atlantic, Bermuda to ca. $8^{\circ} \mathrm{N}$., $44^{\circ} \mathrm{W}$. Pacific off Lower California; in ca. $4^{\circ} \mathrm{S} ., 116^{\circ} \mathrm{W}$.; southwest of Juan Fernandez Island; and northeast of New Zealand, off the Kermadec Islands. Indian Ocean off the Seychelles, the Maldives, south of Sumatra, and in the southern ocean at ca. $50^{\circ} \mathrm{S} ., 123^{\circ} \mathrm{E}$. Probably chiefly deep-abyssal as an adult, leptocephali usually at higher levels. Extreme depth range of adult $800-5100$ meters. 
Length.-68-130 mm. Leptocephali 10-62 mm.

Remarks.-C. atrum is bathypelagic and the only species of the family.

\section{Family HALOSAURIDAE}

Of the twenty described species, eight have been reported from the deep-abyssal zone. All are benthic deep-sea fishes, almost cosmopolitan in distribution between Lat. $40^{\circ} \mathrm{N}$. and $46^{\circ} \mathrm{S}$. Aldrovandia rostrata has also been reported from below 3660 meters. Only A. macrochir can be said to prefer deep-abyssal waters.

\section{Halosaurus johnsonianus Vaillant}

Halosaurus johnsonianus Vaillant, 1888, Exp. Sci. Trav. Talis., Poiss., pp. 31, 181, pl. 15, fig. 2-Atlantic off Soudan, Talisman Station 81, 1139 meters (see footnote, p. 110).

Halosaurus johnsonianus Goode and Bean, 1895, Ocean. Ichth., p. 131, fig. 153; Collett, 1896, Rés. Camp. Sci. Monaco, 10 : 143, pl. 4, fig. 20; Vaillant, in Roule, 1919, op. cit., 52: 130; Koefoed, 1927, Rep. Sci. Res. M. Sars No. Atl. Deep-sea Exp. 1910, 4, (1), p. 67; Fowler, 1936, Bull. Amer. Mus. Nat. Hist., 70: 250, fig. 117; Bertin and Estève, 1951, Cat. Types Poiss., 6: 32; Tucker, 1954, Bull. Brit. Mus. (Nat. Hist.), Zool., 2: 192.

Deep-abyssal records.-Eastern Atlantic off Morocco, 2115, 2104 meters, six specimens in one haul, three in the other.

Distribution.-Eastern Atlantic only, off the Azores, Morocco, Soudan, and the Canary Islands, two deep-abyssal records and 109 specimens reported from sixteen hauls in 834-1590 meters. Perhaps only accidental in deep-abyssal waters.

Length. - 200-408 mm.

\section{Halosaurus attenuatus Garman}

Halosaurus attenuatus Garman, 1899, Mem. Mus. Comp. Zool., 24: 296, pl. 60 , fig. $1-$ Pacific near the Galapagos Islands, ca. $2^{\circ}$ N., $92^{\circ}$ W., 2488 meters.

?Halosaurus attenuatus Townsend and Nichols, 1925, Bull. Amer. Mus. Nat. Hist., 52 : 11.

Deep-abyssal record.-Eastern Pacific, 2488 meters, the type.

Distribution.-Eastern Pacific, one deep-abyssal specimen and one damaged example, doubtfully identified with this species, off Lower California in 1180 meters.

Lenith. $-406 \mathrm{~mm}$. 


\section{Aldrovandia rostrata Günther}

Halosaurus rostratus Günther, 1878, Ann. Mag. Nat. Hist., (5), 2: 252-MidAtlantic, ca. $35^{\circ} \mathrm{N}$., $50^{\circ} \mathrm{W} ., 5029$ meters.

Halosaurus rostratus Günther, 1887, Rep. Sci. Res. Voy. Challenger, Zool., 22: 241 , pl. 59, fig. D; von Lendenfeld, 1887 , op. cit., p. 283 , pl. 73 , figs. $54-64$.

Aldrovandia rostrata Goode and Bean, 1895, Ocean. Ichth., p. 132, fig. 154; Jordan and Evermann, 1896, Bull. U. S. Nat. Mus., 47: 609.

Halosauropsis rostratus Roule, 1919, Rés. Camp. Sci. Monaco, 52: 29; Fowler, 1936, Bull. Amer. Mus. Nat. Hist., 70: 252, fig. 118.

?Aldrovandia sp. Parr, 1937, Bull. Bingham Oceanogr. Coll., 3, (7), p. 60.

Deep-abyssal record.-Mid-Atlantic west of the mid-Atlantic ridge, 5029 meters, the type.

Distribution.-Eastern Atlantic, ca. $15^{\circ} \mathrm{N} ., 23^{\circ} \mathrm{W}$, one specimen, 1311 meters. Mid-Atlantic, one specimen from deep-abyssal waters. Western Atlantic off the Bahama Islands, four specimens in 1645-1729 meters, identification not certain.

Length.-?-508 $\mathrm{mm}$.

Aldrovandia affinis Günther. Table 13.

Halosaurus affinis Günther, 1877, Ann. Mag. Nat. Hist., (4), 20: 444Pacific south of Japan, ca. $34^{\circ}$ N., $138^{\circ}$ E., 1034 meters.

Halosaurus affinis Bleeker, ${ }^{*} 1877-78$, Verh. Akad. Amsterdam, 18: 21; Günther, 1887, Rep. Sci. Res. Voy. Challenger, Zool., 22: 241, pl. 59, fig. B; Alcock, 1890, Ann. Mag. Nat. Hist., (6), 6: 309; 1891, op. cit., (6), 7: 11; Gilchrist, 1906, Mar. Invest. So. Afr., 4: 171; Weber, 1913, Monogr. Siboga Exp., 57: 93; Thompson, *1916, Prov. Cape Good Hope Mar. Biol. Rep., 3: 84; Gilchrist and von Bonde, 1924, Rep. Fish. Mar. Biol. Surv. So. Afr., 3, (7), p. 10.

Halosaurus anguilliformis Alcock, 1889, Ann. Mag. Nat. Hist., (6), 4: 453; 1899, Descr. Cat. Indian Deep-sea Fishes, p. 184.

Halosaurus Hoskynii Alcock, 1890, Ann. Mag. Nat. Hist., (6), 6: 309; 1891, op. cit., (6), 7:11; 1892, Ill. Zool. Investigator, Fishes, pl. 7, fig. 3.

Aldrovandia affinis Goode and Bean, 1895, Ocean. Ichth., p. 516; Barnard, 1925, Ann. So. Afr. Mus., 21 : 167; Norman, 1939, Sci. Rep. John Murray Exp. 1933-34, 7, (1), p. 46; Smith, 1949, Sea Fishes So. Afr., p. 111, fig. 171.

Aldrovandia hoskynii Goode and Bean, 1895, Ocean. Ichth., p. 516.

Aldrovandia anguilliformis Goode and Bean, 1895, loc. cit.

Halosauropsis affinis Weber and de Beaufort, 1922, Fishes Indo-Austr. Arch., 4: 5 .

Deep-abyssal records. - South Africa off Cape Point, 2560 meters, three specimens; 2195 meters, eight specimens; 1847 meters, two specimens. 
North Indian Ocean, ca. $11^{\circ}$ N., $74^{\circ}$ E., 1829 meters, four specimens.

Distribution.- South Africa, off Cape Point, three deep-abyssal records and six specimens in 914-1381 meters. Indian Ocean in the Laccadive Sea, the Gulf of Manaar, near the Maldive Islands and off Zanzibar, one deep-abyssal record and at least three specimens in 1170-1789 meters. Western Pacific south of Japan and in the Timor Sea, four specimens, 883-1034 meters.

The fact that more than two specimens per haul have been found only in deep-abyssal waters suggests that the species may be found chiefly in this area. However, it has been caught too rarely for its habitat to be determined with certainty.

Length. $-310-533 \mathrm{~mm}$.

\section{Aldrovandia gracilis Goode and Bean}

Aldrovandia gracilis Goode and Bean, 1895, Ocean. Ichth., p. 134, fig. 157Gulf of Mexico, 2615 meters.

Aldrovandia gracilis Jordan and Evermann, 1896, Bull. U. S. Nat. Mus., 47 : $610 ; 1900$, op. cit., fig. 263; Zugmayer, in Roule and Angel, 1933, Rés. Camp. Sci. Monaco, 86: 81.

Deep-abyssal records.-Gulf of Mexico, 2615, 2434 meters, two specimens.

Distribution.-Western Atlantic in the Gulf of Mexico, off Guadeloupe Island, and in ca. $42^{\circ} \mathrm{N} ., 63^{\circ} \mathrm{W}$., two deep-abyssal specimens and two in 1408 and 1380 meters.

Length.-- $-490 \mathrm{~mm}$.

\section{Aldrovandia pallida Goode and Bean}

Aldrovandia pallida Goode and Bean, 1895, Ocean. Ichth., p. 135, fig. 158 -Gulf of Mexico, 1747 meters.

Aldrovandia pallida Jordan and Evermann, 1896, Bull. U. S. Nat. Mus., 47: 611.

Deep-abyssal records.-Gulf of Mexico, 2615, 2434 meters, an unstated number of specimens from two hauls.

Distribution.-Western Atlantic only, Gulf of Mexico and $36^{\circ}-$ $41^{\circ} \mathrm{N}$., $65^{\circ}-74^{\circ} \mathrm{W}$., two records from deep-abyssal waters and an unstated number of specimens from seven hauls, three of them between 1747 and 1765 meters, two in 1569 and 1514 meters, and two in 1268 and 1241 meters.

Length.-?-600 $\mathrm{mm}$. 


\section{Aldrovandia macrochir Günther. Table 14.}

Halosaurus macrochir Günther, 1878, Ann. Mag. Nat. Hist., (5), 2: 250-type locality not stated, here designated as Challenger Station V, Atlantic off Strait of Gibraltar, 1994 meters, the locality of the figured specimen in Günther, 1887.

Halosaurus macrochir Goode and Bean, 1883, Bull. Mus. Comp. Zool., 10: 219; Günther, 1887, Rep. Sci. Res. Voy. Challenger, Zool., 22: 232, 237, pl. 59, fig. A, pl. 60, figs. 1-8; von Lendenfeld, 1887, op. cit., p. 283, pl. 73, figs. 45-48; Vaillant, 1888, Exp. Sci. Trav. Talis., Poiss., p. 170, pl. 16, fig. 2 .

Halosaurus goodei Gill, 1883, Proc. U. S. Nat. Mus., 6: 257; Böhlke, 1953, Stanf. Ichth. Bull., 5: 51.

Aldrovandia macrochira (macrochir of authors) Goode and Bean, 1895, Ocean. Ichth., p. 133, fig. 155; Jordan and Evermann, 1896, Bull. U. S. Nat. Mus., 47: 609; 1900, op. cit., fig. 262; Barnard, 1925, Ann. So. Afr. Mus., 21: 168; Zugmayer, in Roule and Angel, 1933, Rés. Camp. Sci. Monaco, 86: 81; Lozano Rey, 1947, Mem. R. Acad. Cien. Madrid, Ser. Cien. Nat., 11: 585, pl. 11, fig. 2, text fig. 170; Smith, 1949, Sea Fishes So. Afr., p. 111.

Aldrovandia goodei Goode and Bean, 1895, Ocean. Ichth., p. 133; Jordan and Evermann, 1896, Bull. U. S. Nat. Mus., 47: 610 .

Halosauropsis macrochir Collett, 1896, Rés. Camp. Sci. Monaco, 10: 146, pl. 4, fig. 23; Zugmayer, 1911, op. cit., 35: 12; Murray and Hjort, 1912, Depths of Ocean, pp. 121, 396, fig. 103, b; Roule, 1919, Rés. Camp. Sci. Monaco, 52: 29; Vaillant, in Roule, 1919, op. cit., p. 130; Koefoed, 1927, Rep. Sci. Res. M. Sars No. Atl. Deep-sea Exp. 1910, 4, (1), p. 65, pl. 4, fig. 7, text figs. 14, 15; Fowler, 1936, Bull. Amer. Mus. Nat. Hist., 70: 253, fig. 119; Nybelin, 1948, Göteborgs K. Vetensk. Vitt.-Samh. Handl., (B), 6, (16), p. 54 .

Halosaurus niger Gilchrist, 1906, Mar. Invest. So. Afr., 4: 170, pl. 51; Gilchrist and von Bonde, 1924, Rep. Fish. Mar. Biol. Surv. So. Afr., 3, (7), p. 10.

Distribution.-Eastern Atlantic from ca. $50^{\circ} \mathrm{N} ., 11^{\circ} \mathrm{W}$. to the Azores and the Canaries, and west to ca. $34^{\circ} \mathrm{N}$., $33^{\circ} \mathrm{W}$. MidAtlantic on the mid-Atlantic ridge, ca. $45^{\circ} \mathrm{N}$., $25^{\circ} \mathrm{W}$. Western Atlantic, $17^{\circ}-41^{\circ}$ N., $65^{\circ}-76^{\circ}$ W. South Africa off Cape Point. South Indian Ocean near Marion Island. Primarily a deep-abyssal species, extreme depth range 1163-3166 meters. Center of distribution probably in about 1800-2400 meters (Table 14).

Length. $-120-650 \mathrm{~mm}$.

\section{Aldrovandia phalacra Vaillant}

Halosaurus phalacrus Vaillant, 1888, Exp. Sci. Trav. Talis., Poiss., p. 185, pl. 15, fig. 3, pl. 16, fig. 1-Atlantic off the Azores, Talisman Station 121, 1442 meters (see footnote, p. 110).

Aldrovandia phalacra Goode and Bean, 1895, Ocean. Ichth., p. 134, fig. 156. 
Halosaurus phalacrus Brauer, 1906, Wiss. Ergebn. Deutschen Tiefsee Exp. Valdivia, 15, (1), p. 253; Zugmayer, 1911, Rés. Camp. Sci. Monaco, 35: 11; Roule, 1919, op. cit., 52: 28; Vaillant, in Roule, 1919, op. cit., p. 130.

Halosauropsis phalacrus Fowler, 1936, Bull. Amer. Mus. Nat. Hist., 70 : 254; Nybelin, 1948, Göteborgs K. Vetensk. Vitt.-Samh. Handl., (B), 5, (16), p. 58; Bertin and Estève, 1951, Cat. Types Poiss., 6: 33.

Aldrovandia phalacrus Lozano Rey, 1947, Mem. R. Acad. Cien. Madrid, Ser. Cien. Nat., 11 : 587, pl. 11, fig. 3.

Deep-abyssal records.-Eastern Atlantic, ca. $43^{\circ}$ N., $9^{\circ}$ W., 2320 meters, one specimen.

Eastern Atlantic off the Azores, 2220 meters, one specimen.

Eastern Atlantic, ca. $35^{\circ}$ N., $8^{\circ}$ W., $2150-2300$ meters, seven specimens in one haul.

Eastern Atlantic off Morocco, 2190, 2104 meters, two specimens.

Distribution.-Eastern Atlantic from ca. $43^{\circ}$ N., $9^{\circ} \mathrm{W}$. and off the Azores to ca. $16^{\circ} \mathrm{N}$., $23^{\circ} \mathrm{W}$., five deep-abyssal records and fourteen specimens from eight hauls in 1103-1786 meters. North Indian Ocean, ca. $1^{\circ} \mathrm{N}$., $45^{\circ} \mathrm{E}$., one specimen, 1644 meters.

Length. $-250-480 \mathrm{~mm}$.

\section{Family NOTACANTHIDAE}

Only four of the nineteen established species of notacanth fishes have been recorded from deep-abyssal waters, and none of the four is represented by more than two specimens. The family is distributed throughout the world except in the Antarctic.

Macdonaldia sp. Koefoed

Macdonaldia sp. Koefoed, 1927, Rep. Sci. Res. M. Sars No. Atl. Deep-sea Exp. 1910,4 , (1), p. 71 , pl. 4, fig. 2 -Atlantic, ca. $34^{\circ} \mathrm{N} ., 33^{\circ} \mathrm{W}$.

Macdonaldia sp. Trotti, 1942, Ann. Mus. Civ. Stor. Nat. Genova, 61 : 278.

Deep-abyssal record and distribution.-Eastern Atlantic, 2615 meters, one specimen.

Length. $-321 \mathrm{~mm}$.

Remarks. - The specimen may be referable to $M$. challengeri Vaillant or to $M$. longus Gill and Townsend, both of which are Pacific forms. The latter, known only from the type, was caught in Bering Sea at a depth of 1805 meters.

\section{Macdonaldia challengeri Vaillant}

Notacanthus challengeri Vaillant, 1888, Exp. Sci. Trav. Talis., Poiss., p. 388 -Pacific off Japan, ca. $34^{\circ}$ N., $140^{\circ}$ E., 3429 meters. 
Notacanthus rissoanus Günther, 1887, Rep. Sci. Res. Voy. Challenger, Zool., 22: 250, pl. 61, fig. B.

Macdonaldia challengeri Goode and Bean, 1895, Ocean. Ichth., p. 172; Jordan and Evermann, 1896, Bull. U. S. Nat. Mus., 47:617; Gilbert, 1896, Rep. U. S. Comm. Fish, 1893: 404; Koefoed, 1927, Rep. Sci. Res. M. Sars No. Atl. Deep-sea Exp. 1910, 4, (1), p. 72; Trotti, 1942, Ann. Mus. Civ. Stor. Nat. Genova, $61: 278$.

Deep-abyssal records and distribution.-Western Pacific, 3429 meters, the type.

North Pacific, Bering Sea, ca. $56^{\circ}$ N., $172^{\circ}$ W., 2971 meters, one specimen.

Length. $-396-508 \mathrm{~mm}$.

\section{Macdonaldia africana Gilchrist and von Bonde}

Macdonaldia africana Gilchrist and von Bonde, 1924, Rep. Fish. Mar. Biol. Surv. So. Afr., 3, (7), p. 11, pl. 3, fig. 1-off Table Bay, South Africa.

Macdonaldia africana Koefoed, 1927, Rep. Sci. Res. M. Sars No. Atl. Deepsea Exp. 1910, 4, (1), p. 72; Trotti, 1942, Ann. Mus. Civ. Stor. Nat. Genova, $61: 278$.

Deep-abyssal record and distribution.--South Africa, 2231 meters, one specimen.

Length. $-395 \mathrm{~mm}$.

Remarks.-J. L. B. Smith $(1949$, p. 110) puts this species in the synonymy of $M$. rostrata Collett, which is known from both sides of the north Atlantic in 923-1797 meters, off South Africa in 1645 meters, and has been taken once off Greenland in 662 meters.

\section{Polyacanthonotus vaillanti Fowler}

Polyacanthonotus vaillanti Fowler, 1934, Proc. Acad. Nat. Sci. Philadelphia, 85: 266, fig. 27-Atlantic off United States coast, ca. $38^{\circ}$ N., $72^{\circ}$ W., 1995 meters.

Notacanthus rissoanus Vaillant, 1888, Exp. Sci. Trav. Talis., Poiss., p. 335, pl. 27, fig. 1.

Polyacanthonotus rissoanus Fowler, 1936, Bull. Amer. Mus. Nat. Hist., 70: 256 (part).

Polyacanthonotus vaillanti Trotti, 1942, Ann. Mus. Civ. Stor. Nat. Genova, $61: 267$.

Deep-abyssal records and distribution.-Eastern Atlantic off Morocco, 2212 meters, one specimen.

Western Atlantic, 1995 meters, the type.

Length. $-260-500 \mathrm{~mm}$. 
Remarks.-The type specimen, in the United States National Museum, bears a label reading "Station No. 2103," for which the correct data are Lat. $38^{\circ} 47^{\prime} 20^{\prime \prime}$ N., Long. $72^{\circ} 37^{\prime}$ W., 1091 fathoms (not $42^{\circ} 04^{\prime} 30^{\prime \prime} \mathrm{N} ., 124^{\circ} 31^{\prime} \mathrm{W}$., 65 fathoms, as stated by Fowler).

\section{Polyacanthonotus altus Gill and Townsend}

Macdonaldia alta Gill and Townsend, 1897, Proc. Biol. Soc. Washington, 11: 232 -Bering Sea, ca. $54^{\circ}$ N., $168^{\circ}$ W.

Macdonaldia alta Jordan and Evermann, 1898, Bull. U. S. Nat. Mus., 47: 2826.

Polyacanthonotus altus Brauer, 1906, Wiss. Ergebn. Deutschen Tiefsee Exp. Valdivia, 15, (1), p. 387.

Deep-abyssal record and distribution.-North Pacific, 2562 meters, one specimen.

Length.-Not stated.

\section{Family MORIDAE}

The family Moridae as recently defined by Svetovidov (1948, p. 67) contains seventeen genera and about seventy species, many of them inhabiting deep water. Only three reach the deep-abyssal zone and none can be said to prefer these depths, although Antimora rostrata is not rare below 2000 meters. Specimens of the bathypelagic genus Melanonus have been taken in nets fishing above as well as below 2000 meters but not in sufficient numbers to warrant its inclusion in the deep-abyssal fauna. Microlepidium grandiceps Garman is apparently bathypelagic also.

The family is represented in all temperate, tropical, and boreal seas.

\section{Lepidion lepidion Risso}

Gadus lepidion Risso, *1810, Ichth. Nice, p. 118, pl. 11, fig. 40--Mediterranean (Nice).

Lota lepidion Risso, 1826, Hist. Nat. Eur. Merid., 3: 218; Canestrini, *1863, Arch. Zool. Anat. Fis. Genova, 2.

Lepidion rissoi Swainson, *1838, Nat. Hist. Classif. Fishes, 1: 319; Goode and Bean, 1895, Ocean. Ichth., p. 370, fig. 323.

Lepidion rubescens Swainson, *1839, Nat. Hist. Classif. Fishes, 2: 307.

Haloporphyrus lepidion Canestrini, *1872, Fauna Ital., 3: 157; Giglioli, 1880, Nature, London, 21 : 202; Vinciguerra, 1883, Ann. Mus. Civ. Stor. Nat. Genova, 18: 554, pl. 3; 1885, op. cit., 22: 459; 1932, op. cit., 56: 15; Günther, 1887, Rep. Sci. Res. Voy. Challenger, Zool., 22: 91; Carus, 1893, Prodr. Faunae Medit., 2: 576; Parona, *1898, Atti Soc. Ligure Sci. 
Nat. Geogr., 9; Vaillant, in Roule, 1919, Rés. Camp. Sci. Monaco, 52: 134.

Haloporphyrus eques Günther, 1887, Rep. Sci. Res. Voy. Challenger, Zool., 22: 91, pl. 18, fig. B; Holt and Calderwood, 1895, Sci. Trans. Roy. Dublin Soc., (2), 5, (9), p. 446, pl. 39, figs. 1, 2; Koehler, 1896, Ann. Univ. Lyon, 26 : 487; Lütken, 1898, Danish Ingolf Exp., 2, (1), p. 30, pl. 4, fig. 7; Ehrenbaum, 1902, Fauna Arct., 2: 114; *1936, Naturg. wirtsch. Bedeut. Seefische Nordeur., p. 129; Holt and Byrne, 1906, Rep. Fish. Ireland 1905, Sci. Invest., 2: 24; Saemundsson, *1927, Syn. Fishes Iceland; 1949, Zool. Iceland, 4, (72), p. 71 (further refs., Iceland); Hickling, 1928, Ann. Mag. Nat. Hist., (10), 2: 196; Schnakenbeck, 1933, Faune Ichth. Atl. Nord, 12, fig.; Jensen, 1948, Skr. Univ. Zool. Mus. København, 9: 174.

Lepidion eques Goode and Bean, 1895, Ocean. Ichth., p. 371; Collett, 1905, Rep. Norwegian Fish. Mar. Invest., 2, (3), p. 69; Murray and Hjort, 1912, Depths of Ocean, pp. 400, 401, 433, fig. 280; Koefoed, 1927, Rep. Sci. Res. M. Sars No. Atl. Deep-sea Exp. 1910, 4, (1), p. 124, fig. 50; Norman, 1935, Disc. Rep., 12: 50.

Haloporphyrus lepidion var. eques Roule, 1919, Rés. Camp. Sci. Monaco, 52: 78 .

Lepidion lepidion Norman, 1935, Disc. Rep., 12: 50; Tortonese and Trotti, 1949, Atti Accad. Ligure Sci. Lett., 6, (1), p. 57.

Deep-abyssal records.-Mediterranean, ca. $43^{\circ}$ N., $7^{\circ}$ E., 2230 , 2170 meters, two specimens, both from traps.

Distribution.-Eastern and north Atlantic from Davis Strait (ca. $63^{\circ} \mathrm{N}$.), southwest of Iceland (ca. $64^{\circ}$ N.) and the FaroeShetland Channel, south to the Gulf of Gascony. Western Mediterranean. Not a deep-abyssal species except in the Mediterranean. Depth range [216] (see Hickling, 1928, p. 196) and 540-1804 meters. Center of distribution probably in about 650-1000 meters, where it has been taken at times in large numbers.

Length.-70-889 $\mathrm{mm}$.

Remarks. - There may be two species included here under the name Lepidion lepidion. If distinct, the two forms are closely related and the various records cannot be applied correctly to one or the other from a study of the literature alone.

\section{Antimora rostrata Günther. Table 15.}

Haloporphyrus rostratus Günther, 1878, Ann. Mag. Nat. Hist., (5), 2: 18 - type locality not stated, here designated as Challenger Station no. 320, off Montevideo, ca. $37^{\circ}$ S., $53^{\circ}$ W., 1098 meters, the locality of the specimen figured by Günther (1887).

Haloporphyrus viola Goode and Bean, 1878, Proc. U. S. Nat. Mus., 1: 257; 1879, Bull. Essex Inst., 11: 8; 1883, Bull. Mus. Comp. Zool., 10: 206; Goode, 1880, Proc. U. S. Nat. Mus., 3: 476. 
Antimora viola Jordan, 1887, Rep. U. S. Fish Comm., 1885: 917; Günther, 1887, Rep. Sci. Res. Voy. Challenger, Zool., 22: 94, pl. 15; Goode and Bean, 1895, Ocean. Ichth., p. 372, fig. 324; Collett, 1896, Rés. Camp. Sci. Monaco, 10: 59; Jordan and Evermann, 1896, Bull. U. S. Nat. Mus., $47: 2544 ; 1900$, op. cit., figs. 893, 893, $a$; Lütken, 1898, Danish Ingolf Exp., 2, (1), p. 30; Ehrenbaum, 1902, Fauna Arct., 2: 115; Holt and Byrne, 1906, Rep. Fish. Ireland 1905, Sci. Invest., 2: 25; Murray and Hjort, 1912, Depths of Ocean, pp. 121, 400, fig. 279; Waite, 1916, Sci. Rep. Austr. Antarctic Exp. 1911-1914, (C), 3: 76; Gilchrist and von Bonde, 1924, Rep. Fish. Mar. Biol. Surv. So. Afr., 3, (7), p. 13; Bigelow and Welsh, 1925, Bull. U. S. Bur. Fish., 60, (1), p. 444, fig. 218; McCulloch, 1929-30, Mem. Austr. Mus., 5: 129; Zugmayer, in Roule and Angel, 1933, Rés. Camp. Sci. Monaco, 86: 82; Scholander and van Dam, 1954, Biol. Bull., $107: 249$.

Antimora rostrata Günther, 1887, Rep. Sci. Res. Voy. Challenger, Zool., 22: 93, pl. 16, fig. A; Goode and Bean, 1895, Ocean. Ichth., p. 375; Zugmayer, 1911, Rés. Camp. Sci. Monaco, 35: 122; Zugmayer, in Roule and Angel, 1933, op. cit., 86: 82; Koefoed, 1927, Rep. Sci. Res. M. Sars No. Atl. Deepsea Exp. 1910, 4, (1), p. 118, figs. 44-46; Schroeder, 1940, Copeia, p. 236; Clemens and Wilby, 1946, Bull. Fish. Res. Bd. Canada, 68: 133, fig. 75; Jensen, 1948, Skr. Univ. Zool. Mus. København, 9: 173; Bigelow and Schroeder, 1953, Fish. Bull., Fish Wildlife Serv., 53, (74), p. 233, fig. 112. Antimora microlepis Bean, 1890, Proc. U. S. Nat. Mus., 13: 38; Goode and Bean, 1895, Ocean. Ichth., p. 531; Gilbert, 1896, Rep. U. S. Comm. Fish, 1893: 456, 473; 1905, Bull. U. S. Fish Comm., 23: 656; Jordan and Evermann, 1898, Bull. U. S. Nat. Mus., 47: 2545; Jordan and Gilbert, 1899, Rep. Fur-seal Invest., 3 : 487; Gilbert and Burke, 1912, Bull. Bur. Fish., 30 : 90; Townsend and Nichols, 1925, Bull. Amer. Mus. Nat. Hist., 52: 16; Schmidt, 1930, Proc.4th Pac. Sci. Congr., 3: 461; *1931, Akad. Sci. U.S.S.R., Trans. Pac. Comm., 2: 151; Soldatov and Lindberg, 1930, Bull. Pac. Sci. Fish. Inst., 5 : 516; Svetovidov, 1948, Fauna U.S.S.R., Fishes, 9, (4), p. 69, pl. 18 , fig. 2 .

Antimora rhina Garman, 1899, Mem. Mus. Comp. Zool., 24: 185.

Antimora mikrolepis Franz, 1910, Abh. Bayer. Akad. Wiss., 4, Suppl., p. 29. Antimora australis Barnard, 1925, Ann. Mag. Nat. Hist., (9), 15: 499; 1925, Ann. So. Afr. Mus., 21 : 321; Smith, 1949, Sea Fishes So. Afr., p. 138.

Distribution.-North Atlantic from Davis Strait (ca. $64^{\circ} \mathrm{N}$.) and $46^{\circ}-33^{\circ} \mathrm{N}$. in the west and from ca. $64^{\circ} \mathrm{N}$. to ca. $35^{\circ} \mathrm{N}$., $8^{\circ} \mathrm{W}$. in the east. Southwestern Atlantic, ca. $37^{\circ} \mathrm{S} ., 53^{\circ} \mathrm{W}$. South Africa off Cape Point. Southwestern Pacific off Tasmania. Western Pacific off Japan. Mid-Pacific off the Hawaiian Islands. Eastern and North Pacific from the Gulf of Panama $\left(7^{\circ} \mathrm{N}\right.$.) northward to Alaska and across Bering Sea $\left(54^{\circ}\right.$ N.) to Kamchatka (ca. $52^{\circ}$ N., $158^{\circ}$ E.). A relatively common species with a wide vertical range (403-2904 meters). Probably not uncommon in deep-abyssal waters but with the center of distribution in ca. 800 1800 meters (Table 15). 


\section{Length.-140-610 mm.}

Remarks.-Gilchrist and von Bonde (1924, p. 13) listed a number of specimens from four hauls off South Africa. One of these lots, no. 3349, from Pickle Station 353, in 318 meters, had been listed earlier as "Callionymus" (Gilchrist, 1922, p. 19). In this same list Gilchrist included the following catches that were not mentioned in 1924 by Gilchrist and von Bonde: Station 347, eight specimens in 1225 meters; Station 354, seven specimens in 1145 meters; Station 519, ten specimens in 1098 meters; Station 522, thirteen specimens in 2194 meters; Station 542, one specimen in 1060 meters; and Station 543 , one specimen in 1802 meters. These records have been included in Table 15, and the record from 318 meters has been omitted.

\section{Laemonema melanurum Goode and Bean}

Laemonema melanurum Goode and Bean, 1895, Ocean. Ichth., p. 363, fig. 316 -Atlantic off northern Florida, 805 meters.

Laemonema melanurum Jordan and Evermann, 1896, Bull. U. S. Nat. Mus., 47: 2557; 1900, op. cit., fig. 905; Zugmayer, in Roule and Angel, 1933, Rés. Camp. Sci. Monaco, 86: 84; ?Parr, 1937, Bull. Bingham Oceanogr. Coll., 3, (7), p. 61.

Deep-abyssal records.-Gulf of Mexico, 2683 meters, (?)specimens.

?Off Bermuda, 2012 meters, one young specimen, doubtfully referred to this species.

Distribution.-Western Atlantic, two deep-abyssal records and an unstated number of specimens from one haul in 1733 meters (ca. $39^{\circ} \mathrm{N} ., 69^{\circ}$ W.) and from six hauls in $380-805$ meters (ca. $11^{\circ}$ N., $69^{\circ}$ W.; Gulf of Mexico; and $30^{\circ}-31^{\circ}$ N., $79^{\circ}$ W.). Eastern Atlantic, ca. $47^{\circ} \mathrm{N}$., $7^{\circ} \mathrm{W}$., one specimen in 920 meters. Probably accidental in deep-abyssal waters.

Length. $-73-330 \mathrm{~mm}$.

\section{Family GADIDAE}

The family Gadidae contains both surface and deep-sea forms, but only one species is known from deep-abyssal waters. Gaidropsarus argentatus Reinhardt has been omitted from the present discussion. Lütken $(1898$, p. 29) recorded some young taken at a depth of 2261 meters in Denmark Strait, but this Arctic-north Atlantic species has never otherwise been reported below 1428 meters. It is common down to 400 meters. Lütken's young specimens could have entered the net in intermediate waters. 
Geographically, gadids are distributed in all seas but are found chiefly in the northern hemisphere.

\section{Gaidropsarus ensis Reinhardt}

Motella ensis Reinhardt, ${ }^{*} 1838$, Vidensk. Selsk. Skr. Nat. Math. Afh., 7 : 116, 128-off Umanak, Greenland, from seal stomach.

Onos (Onus of authors) ensis Gill, 1862, Proc. Acad. Nat. Sci. Philadelphia, 15: 241; Collett, 1880, Norske Nordhavs Exp. 1876-78, Zool., 3: 134; *1891, Vidensk. Selsk. Forh., 1891, (11), p. 16; Günther, 1887, Rep. Sci. Res. Voy. Challenger, Zool., 22: 98; Goode and Bean, 1895, Ocean. Ichth., p. 381, fig. 327; Ehrenbaum, 1902, Fauna Arct., 2: 114; Zugmayer, in Roule and Angel, 1933, Rés. Camp. Sci. Monaco, 86: 84; Schnakenbeck, 1933, Faune Ichth. Atl. Nord, 12, fig.

Motella ensis Collett, *1878, Vidensk. Selsk. Forh., 1878, (17); Lütken, 1882, Vidensk. Medd. Dansk. naturh. Foren., 1881 : 232, 236.

Onus rufus Gill, 1883, Proc. U. S. Nat. Mus., 6: 259.

Gaidropsarus ensis Jordan and Evermann, 1898, Bull. U. S. Nat. Mus., 47:

2558; Jensen, 1948, Skr. Univ. Zool. Mus. København, 9: 166, pl. 4,

fig. a; Svetovidov, 1948, Fauna U.S.S.R., Fishes, 9, (4), p. 88.

Onogadus ensis de Buen, 1934, Bol. Soc. Esp. Hist. Nat., 34: 500.

Deep-abyssal records.-Western Atlantic off United States coast, $39^{\circ}-40^{\circ}$ N., 2022 meters, one and (?)specimens from two hauls.

Western Atlantic off United States coast, ca. $39^{\circ}$ N., 1908, 1977 meters, two hauls, number of specimens not stated.

Distribution.-Western Atlantic, four deep-abyssal records in ca. $39^{\circ}-40^{\circ} \mathrm{N}$.; and from ca. $65^{\circ} \mathrm{N}$. to $74^{\circ} \mathrm{N}$. (Baffin Bay), three specimens from an unknown depth, five in $360-500$ meters and sixty-five from three hauls made in 1200,1500 and 1600 meters. Perhaps deep-abyssal in the southern part of its range.

Length. $-310-400 \mathrm{~mm}$.

Remarks. - The specimen reported by Zugmayer (1933, p. 84) from near Halifax in 25 meters should be re-examined.

\section{Family MACROURIDAE}

Macrourids, the most characteristic and abundant of all benthic deep-sea fishes, are found in all seas and at all depths, with the conspicuous exception of the Polar Basin. Apparently the only macrourids reported from waters of negative temperatures are three Antarctic species, all of them deep-abyssal: Nematonurus lecointei Dollo, one specimen reported at a temperature of $31^{\circ} \mathrm{F}$., the type in $+0.5^{\circ} \mathrm{C}$.; Chalinura ferrieri Regan, $31.9^{\circ} \mathrm{F}$. and $-0.3^{\circ} \mathrm{C}$.; and C. whitsoni Regan, $31.9^{\circ} \mathrm{F}$. and $-0.2^{\circ} \mathrm{C}$. 
Including the ten from the abyssal plain, fifty-four species have been recorded from deep-abyssal waters; only about nine of these species seem to prefer this zone. Twenty-two species are known only at these great depths but from too few specimens for evaluation. Nybelin $(1951$, pp. 13, 14) also reported seven specimens of Chalinura, perhaps representing a new species, from depths between 4255 and 4872 meters in the eastern Atlantic (ca. $29^{\circ} \mathrm{N} ., 17^{\circ} \mathrm{W}$. and ca. $22^{\circ} \mathrm{N}$., $23^{\circ} \mathrm{W}$.).

Nematonurus firmisquamis Gill and Townsend is omitted from the list of deep-abyssal species since no authority has been found for Brauer's listing of the type from 3239 meters (1771 fathoms) (Brauer, 1906, p. 390). The type description contains no station number and no information on depth of capture, nor are there any such data with the type specimen, which is deposited in the United States National Museum. Since the species has been recorded only once again (Evermann and Goldsborough, 1907, p. 349), also from Bering Sea, at a depth of 35 meters, still more doubt attends the truth of Brauer's listing.

Brauer (loc. cit.) also listed in 3239 meters $N$. magnus Gill and Townsend, a species since synonymized with $N$. pectoralis Gilbert. Again no authority has been found for this depth. $N$. pectoralis is a north Pacific species known from several specimens taken in depths ranging between about 180 and 1600 meters.

Jensen (1948, p. 180) has disproved the one deep-abyssal record of Macrourus fabricii Sundevall (Macrourus berglax Lacépède).

Lyconus pinnatus Günther (1887, p. 158) has also been omitted from the present discussion because the three small $(25-41 \mathrm{~mm}$.) specimens taken from nets fished in the deep-abyssal zone were caught in mid-water. Some young macrourids are known to be bathypelagic.

The following two species, having been caught only in nets fishing far above bottom, are apparently adapted for life in intermediate waters as adults also. Neither one can be placed with certainty in the deep-abyssal zone, owing to their infrequent capture.

Cynomacrurus pirei Dollo, Antarctic, South Atlantic, and South Indian, seven specimens, 1025-2000 meters. Length 73-300 mm.

Odontomacrurus murrayi Norman, North Indian Ocean, Arabian Sea, one specimen in 2500 meters. Length ca. $550 \mathrm{~mm}$.

Generic names used in this paper for species of the MacrourusCoryphaenoides group are principally after Gilbert and Hubbs (1916, 1920) and Parr (1946). The taxonomic complexities of these genera 
are beyond the scope of a study undertaken on the basis of the literature alone.

\section{Bathygadus melanobranchus Vaillant}

Bathygadus melanobranchus Vaillant, 1888, Exp. Sci. Trav. Talis., Poiss., pp. 31, 206, pl. 18, fig. 1-Atlantic off North Africa and the Canary Islands (type locality not designated; see footnote, p. 110).

Bathygadus melanobranchus Goode and Bean, 1895, Ocean. Ichth., p. 424; Collett, 1896, Rés. Camp. Sci. Monaco, 10: 188; Holt and Byrne, 1908, Rep. Fish. Ireland 1906, Sci. Invest., 5: 57; Murray and Hjort, 1912, Depths of Ocean, pp. 399, 769, fig. 563; Roule, 1919, Rés. Camp. Sci. Monaco, 52: 89; Gilbert and Hubbs, 1920, Bull. U. S. Nat. Mus., 100, (1), p. 388; Farran, 1924, Proc. Roy. Irish Acad., B 36: 120, fig. 9; Gilchrist and von Bonde, 1924, Rep. Fish. Mar. Biol. Surv. So. Afr., 3, (7), p. 12; Barnard, 1925, Ann. So. Afr. Mus., 21 : 334; Koefoed, 1927, Rep. Sci. Res. M. Sars No. Atl. Deep-sea Exp. 1910, 4, (1), p. 113; Schnakenbeck, 1932, Faune Ichth. Atl. Nord, 12, fig.; Roule and Angel, 1933, Rés. Camp. Sci. Monaco, 86: 62; Fowler, 1936, Bull. Amer. Mus. Nat. Hist., 70: 451, fig. 220; Smith; 1949, Sea Fishes So. Afr., p. 136.

Deep-abyssal records. - South Africa off Cape Point, ca. $33^{\circ}$ S., $16^{\circ}$ E., 2560, 2195 meters, eight specimens in two hauls.

Distribution.-Eastern Atlantic, $15^{\circ}-51^{\circ} \mathrm{N}$., $7^{\circ}-33^{\circ} \mathrm{W} ., 231$ specimens known from thirty-four hauls, 830-1700 meters. South Africa off Cape Point and Table Bay, ca. $31^{\circ}-33^{\circ}$ S., $16^{\circ}$ E., eight deepabyssal specimens and ten in 1225 meters; off Natal, ca. $30^{\circ} \mathrm{S}$., $31^{\circ}$ E., twenty-seven specimens, 940 meters. Center of distribution in 800-1400 meters. Probably accidental in deep-abyssal waters.

Length. $-132-440 \mathrm{~mm}$.

Remarks. $-B$. melanobranchus is very close to, if not identical with, the Indo-Pacific species $B$. furvescens Alcock, and the South African examples listed above might belong to either species. Gilchrist and von Bonde (1924, p. 12) followed Brauer (1906, p. 272) in placing furvescens in the synonymy of melanobranchus and did not describe their specimens. The two forms have a similar vertical distribution, although furvescens has been taken at times above 800 meters.

\section{Bathygadus favosus Goode and Bean}

Bathygadus farosus Goode and Bean, 1886, Bull. Mus. Comp. Zool., 12: 160 -Atlantic off Martinique, 863 meters.

Bathygadus favosus Goode and Bean, 1895, Ocean. Ichth., p. 420, fig. 352; Jordan and Everman, 1898, Bull. U. S. Nat. Mus., 47: 2565; 1900, op. cit., fig. 908; Parr, 1946, Bull. Bingham Oceanogr. Coll., 10, (1), p. 12, fig. 4 . 
Bathygadus sp. (favosus?) Koefoed, 1927, Rep. Sci. Res. M. Sars No. Atl. Deep-sea Exp. 1910, 4, (1), p. 114.

Deep-abyssal record.-Gulf of Mexico, 2745 meters, one young specimen.

Distribution.-Western Atlantic in the Caribbean near Martinique, five specimens in two hauls in 863 and 1249 meters; in the Gulf of Mexico, one deep-abyssal record and an unstated number of specimens from two hauls in 768 and 1324 meters. ?Eastern Atlantic, ca. $35^{\circ} \mathrm{N} ., 7^{\circ} \mathrm{W} ., 1615$ meters, a specimen in poor condition doubtfully referred to this species.

Length.-215-350 $\mathrm{mm}$.

Remarks.-Goode and Bean (1895, p. 421) were apparently in error when they stated that the type of this species was taken at Blake Station 80, although their data are otherwise correct. According to a written communication from the Museum of Comparative Zoology the label with the type specimen reads "Off Martinique, 472 fathoms. Coll. Blake Exp. 1878-79." In the station lists compiled by Sanderson Smith (1888, p. 964) only Station 200 was at a depth of 472 fathoms off Martinique. Station 80 was northwest of Cuba in 1222 fathoms.

\section{Gadomus longifilis Goode and Bean}

Bathygadus longifilis Goode and Bean, 1885, Proc. U. S. Nat. Mus., 8: 599 -Gulf of Mexico, 1324 meters.

Bathygadus longifilis Günther, 1887, Rep. Sci. Res. Voy. Challenger, Zool., 22: 157; Goode and Bean, 1895, Ocean. Ichth., p. 422; Collett, 1896, Rés. Camp. Sci. Monaco, 10: 90; Jordan and Evermann, 1898, Bull. U. S. Nat. Mus., 47: 2566; Murray and Hjort, 1912, Depths of Ocean, p. 399, fig. 273; Roule, 1919, Rés. Camp. Sci. Monaco, 52: 88; Koefoed, 1927, Rep. Sci. Res. M. Sars No. Atl. Deep-sea Exp. 1910, 4, (1), p. 116.

Hymenocephalus longifilis Vaillant, 1888, Exp. Sci. Trav. Talis., Poiss., p. 218, pl. 23, fig. 1.

Gadomus longifilis Regan, 1903, Ann. Mag. Nat. Hist., (7), 11: 459; Gilbert and Hubbs, 1920, Bull. U. S. Nat. Mus., 100, (1), p. 391; Fowler, 1936, Bull. Amer. Mus. Nat. Hist., 70 : 452, fig. 221; Parr, 1946, Bull. Bingham Oceanogr. Coll., 10, (1), p. 10, fig. 3.

Deep-abyssal record. -Eastern Atlantic off Morocco, ca. $31^{\circ} \mathrm{N}$., $10^{\circ} \mathrm{W} ., 2165$ meters, one specimen.

Distribution.-Western Atlantic, only in the Gulf of Mexico, at least seventeen specimens ${ }^{1}$ taken between 631-914 and 1351

${ }^{1}$ Including twelve hitherto unrecorded examples taken by the United States Fish and Wildlife Service Research Vessel Oregon at Station $349,29^{\circ} 09^{\prime}$ N., $87^{\circ} 58^{\prime}$ W., 470-500 fathoms, May 22, 1951, seven specimens; and Station $640,29^{\circ} 01^{\prime} \mathrm{N}$., $88^{\circ} 24^{\prime}$ W., 355-475 fathoms, Sept. 19, 1952, five specimens. 
meters. Eastern Atlantic off the Azores, Portugal, Morocco, the Canaries and Soudan, one deep-abyssal specimen and seventy-six in 1084-1635 meters.

\section{Length.-70-292 mm.}

Remarks.-Indo-Pacific specimens identified with this species were referred to other forms by Gilbert and Hubbs (1920, p. 391), who also stated that the eastern Atlantic records were not,actually longifilis. Parr (1946, p. 10) accepted the species as cosmopolitan. Whatever the geographical range may be it is extremely doubtful that the species is more than accidental in the deep-abyssal zone.

\section{Nematonurus cyclolepis Gilbert}

Nematonurus cyclolepis Gilbert, 1896, Rep. U. S. Comm. Fish, 1893: 458Pacific off the Queen Charlotte Islands.

Moseleya cyclolepis Jordan and Evermann, 1898, Bull. U. S. Nat. Mus., 47: 2570.

Dolloa cyclolepis Jordan, Evermann and Clark, 1930, Rep. U. S. Comm. Fish., 1928, (2), p. 203; Clemens and Wilby, 1946, Bull. Fish. Res. Bd. Canada, 68: 134 , fig. 76 .

Nematonurus cyclolepis Böhlke, 1953, Stanf. Ichth. Bull., 5: 59.

Deep-abyssal record and distribution.-Eastern Pacific, 2904 meters, two specimens.

Length.-?-150 mm.

\section{Nematonurus lecointei Dollo}

Macrurus Lecointei Dollo, 1900, Bull. Acad. roy. Belg., (3), 38: 384-Antarctic, ca. $70^{\circ} \mathrm{S},{ }^{\circ} 102^{\circ} \mathrm{W} ., 2800$ meters.

Nematonurus lecointei Dollo, 1904, Res. Voy. Belgica, Zool., Poiss., p. 44, pl. 7; 1909, Proc. Roy. Soc. Edinburgh, 29: 488; Regan, 1913, Trans. Roy. Soc. Edinburgh, 49: 235; Gilbert and Hubbs, 1916, Proc. U. S. Nat. Mus., 51 : 143.

Deep-abyssal records and distribution.-Antarctic, ca. $62^{\circ} \mathrm{S}$., $41^{\circ} \mathrm{W} ., 3246$ meters, one specimen.

Antarctic, ca. $70^{\circ}$ S., $102^{\circ} \mathrm{W}$., 2800 meters, one specimen.

South Atlantic, ca. $48^{\circ} \mathrm{S} ., 10^{\circ} \mathrm{W}$., $3185^{\circ}$ meters, one specimen.

Length.-?-428 $\mathrm{mm}$.

\section{Nematonurus suborbitalis Gill and Townsend}

Macrourus (Nematonurus) suborbitalis Gill and Townsend, 1897, Proc. Biol. Soc. Washington, 11 : $234-$ Bering Sea, ca. $55^{\circ} \mathrm{N} ., 170^{\circ} \mathrm{W}$.

Nematonurus suborbitalis Jordan and Evermann, 1898, Bull. U. S. Nat. Mus., 47: 2572; Gilbert and Hubbs, 1916, Proc. U. S. Nat. Mus., 51 : 143. 
Deep-abyssal record and distribution.-Bering Sea, 3239 meters, one specimen.

Length. $-508 \mathrm{~mm}$.

\section{Nematonurus lepturus Gill and Townsend}

Macrourus lepturus Gill and Townsend, 1897, Proc. Biol. Soc. Washington, 11 : 233 -Bering Sea, ca. $54^{\circ}$ N., $168^{\circ}$ W., 2562 meters.

Macrourus dorsalis Gill and Townsend, 1897, op. cit., p. 233.

Macrourus lepturus Jordan and Evermann, 1898, Bull. U. S. Nat. Mus., 47 : 2584; Jordan and Gilbert, 1899, Rep. Fur-seal Invest., 3: 487; Gilbert and Burke, 1912, Bull. U. S. Bur. Fish., 30: 91.

Nematonurus lepturus Gilbert and Hubbs, 1916, Proc. U. S. Nat. Mus., 51 : 162.

Deep-abyssal records and distribution.-Bering Sea, ca. $54^{\circ} \mathrm{N}$, $168^{\circ}$ W., 2562 meters, two specimens.

Bering Sea, ca. $53^{\circ} \mathrm{N} ., 171^{\circ} \mathrm{W} ., 2226,2065$ meters, two specimens. Length. $-375-660 \mathrm{~mm}$.

\section{Nematonurus abyssorum Gilbert}

Nematonurus abyssorum Gilbert, 1915, Proc. U. S. Nat. Mus., 48: 374, pl. 21 - Pacific off Santa Catalina Island.

Nematonurus abyssorum Gilbert and Hubbs, 1916, Proc. U. S. Nat. Mus., 51 : 143.

Deep-abyssal record and distribution.-Eastern Pacific, 2469-3991 meters, one specimen.

Length. $-803 \mathrm{~mm}$.

Nematonurus armatus Hector. Table 16.

Macrurus armatus Hector, 1875, Ann. Mag. Nat. Hist., (4), 15: 81; 1875, Trans. New Zealand Inst., $7: 249$, pl. 11-off Cape Farewell, New Zealand, 732 meters.

Coryphaenoides affinis Günther, 1878, Ann. Mag. Nat. Hist., (5), 2: 27.

Coryphaenoides variabilis Günther, 1878, op. cit., p. 27.

Macrurus asper Goode and Bean, 1883, Bull. Mus. Comp. Zool., 10 : 196.

Macrurus goodii (goodei of authors) Günther, 1887, Rep. Sci. Res. Voy. Challenger, Zool., 22: 136; Schnakenbeck, 1932, Faune Ichth. Atl. Nord, 12, fig.; Jensen, 1948, Skr. Univ. Zool. Mus. København, 9: 182.

Macrurus armatus Günther, 1887, Rep. Sci. Res. Voy. Challenger, Zool., 22: 150, pl. 40, fig. A; Fowler, 1936, Bull. Amer. Mus. Nat. Hist., 70: 455, fig. 222 .

Macrurus affinis Günther, 1887, Rep. Sci. Res. Voy. Challenger, Zool., 22: 151 , pl. 40 , fig. B. 
Coryphaenoides gigas Vaillant, 1888, Exp. Sci. Trav. Talis., Poiss., p. 232, pl. 20, fig. 2.

Hymenocephalus goodei Goode and Bean, 1895, Ocean. Ichth., p. 407, fig. 340.

Nematonurus armatus Goode and Bean, 1895, Ocean. Ichth., p. 416; Regan, 1914, Brit. Antarctic (Terra Nova) Exp. 1910, Zool., 1: 39; Gilbert and Hubbs, 1916, Proc. U. S. Nat. Mus., 51 : 143; Waite, 1916, Sci. Rep. Austr. Antarctic Exp. 1911-1914, (C), 3: 76; 1921, Rec. So. Austr. Mus., 2, (1), p. 65, fig. 99; McCulloch, 1929-30, Mem. Austr. Mus., 5: 127; Fraser-Brunner, 1935, Proc. Roy. Irish Acad., B42, (9), p. 322; Schroeder, 1940, Copeia, p. 233; Parr, 1946, Bull. Bingham Oceanogr. Coll., 10, (1), pp. 9, 54, figs. 1, 17; Nybelin, 1951, Rep. Swedish Deep-sea Exp. 1947-48, Zool., 2, (1), pp. 13, 24.

Nematonurus affinis Goode and Bean, 1895, Ocean. Ichth., p. 416; Gilbert and Hubbs, 1916, Proc. U. S. Nat. Mus., 51 : 143; Fraser-Brunner, 1935, Proc. Roy. Irish Acad., B42, (9), p. 322.

Nematonurus gigas Goode and Bean, 1895, Ocean. Ichth., p. 416 (not fig. 346); Gilbert and Hubbs, 1916, Proc. U. S. Nat. Mus., 51 : 143; Roule, 1916, Bull. Inst. Océanogr. Monaco, 320: 21; 1919, Rés. Camp. Sci. Monaco, 52: 87, pl. 3, fig. 1; Vaillant, in Roule, 1919, op. cit., p. 134; Farran, 1924, Proc. Roy. Irish Acad., B36: 118.

Macrurus (Hymenocephalus) goodei Lütken, 1898, Danish Ingolf Exp., 2, (1), p. 26.

Nematonurus goodei Jordan and Evermann, 1898, Bull. U. S. Nat. Mus., 47 : 2571; Gilbert and Hubbs, 1916, Proc. U. S. Nat. Mus., 51: 143.

Macrurus (Nematonurus) goodei Ehrenbaum, 1902, Fauna Arct., 2: 117.

Macrurus (Nematonurus) armatus Murray and Hjort, 1912, Depths of Ocean, p. 398, fig. 272; Koefoed, 1927, Rep. Sci. Res. M. Sars No. Atl. Deep-sea Exp. 1910,4 , (1), p. 109, fig. 38 .

Distribution.-Eastern Atlantic in Denmark Strait and ca. $49^{\circ}-$ $27^{\circ} \mathrm{N} ., 5^{\circ}-33^{\circ} \mathrm{W}$. Mid-Atlantic on the mid-Atlantic ridge (ca. $45^{\circ}$ N., $25^{\circ}$ W.) and west of it (ca. $40^{\circ}$ N., $35^{\circ}$ W.). Western Atlantic in Davis Strait, $39^{\circ}-41^{\circ} \mathrm{N}$, and off Havana. South Atlantic off Montevideo. Pacific in ca. $36^{\circ}$ N., $178^{\circ}$ E.; ca. $0^{\circ} 33^{\prime}$ S., $151^{\circ} \mathrm{W}$.; off New Zealand; and off Kangaroo Island, South Australia. South Indian Ocean in ca. $46^{\circ} \mathrm{S} ., 45^{\circ}$ and $48^{\circ}$ E.; and $50^{\circ} \mathrm{S} ., 108^{\circ}$ and $123^{\circ} \mathrm{E}$.

$N$. armatus is one of the few fishes known from more than one or two hauls made on the abyssal plain, below 3660 meters, where twelve specimens have been taken. Its center of distribution may be higher. At least forty-six of the more than seventy-six specimens recorded were caught between about 2600 and 3660 meters. The number of specimens per haul is unknown in many of the western Atlantic records, most of which were in depths of 2200-2600 meters. The species could be more common below 3660 meters than avail- 
able information indicates, as relatively few hauls have been made on the abyssal plain.

There are only three records from above the deep-abyssal zone: in the western Atlantic off Havana in 282 meters (number of specimens not reported) and in ca. $40^{\circ} \mathrm{N} ., 68^{\circ} \mathrm{W}$. in 556 meters (one specimen); and off New Zealand in 732 meters (one specimen). The species is probably accidental at such depths. Extreme depth range $282-4700$ meters.

Length. $-127-805 \mathrm{~mm}$.

\section{Coryphaenoides rupestris Gunnerus}

Coryphaenoides rupestris Gunnerus, *1765, Trondhjem Selsk. Skr., 3: 50, pl. 3, figs. 1, 2-Atlantic off Norway.

Coryphaenoides rupestris Collins, Bean and Rathbun, 1887, Bull. U. S. Fish. Comm., 7: 243; Collett, 1903, Vidensk. Selsk. Forh., 1903, (9), p. 71; 1905, Rep. Norwegian Fish. Mar. Invest., 2, (3), p. 52; Holt and Byrne, 1906, Rep. Fish. Ireland 1905, Sci. Invest., 2: 25; Johnsen, 1921, Bergens Mus. Aarb. 1918-19, (6), p. 48, pl., figs. 3, 4; Hickling, 1928, Ann. Mag. Nat. Hist., (10), 2: 196; Parr, 1946, Bull. Bingham Oceanogr. Coll., 10, (1), pp. 50, 74, figs. 23, 24 (full synonymy); Jensen, 1948, Skr. Univ. Zool. Mus. København, 9: 177; Rae and Wilson, 1953, Scottish Nat., 65: 144; Scholander and Van Dam, 1953, Biol. Bull., 104: 76; 1954, op. cit., 107: 249.

Macrurus rupestris Smitt, 1895, Skand. Fiskar, p. 590, pl. 27A, fig. 2; Schnakenbeck, 1931, Faune Ichth. Atl. Nord, 7, 3 figs.; Ehrenbaum, *1936, Naturg. wirtsch. Bedeut. Seefische Nordeur., p. 130, fig. 102; Saemundsson, 1949, Zool. Iceland, 4, (72), p. 74 (further refs., Iceland).

Macrurus (Coryphaenoides) rupestris Knipowitsch, 1926, Trans. Inst. Sci. Expl. North, 27: 149, fig. 114; Rae and Wilson, 1952, Cons. Perm. Int. Expl. Mer, Ann. Biol., 9: 39; Andriashev, 1954, Fauna S.S.S.R., 53: 195, 534, fig. 96.

Macrurid larva "A.H." $1 a$ and "A.H." 2 Johnsen, 1927, Nyt. Mag. Naturv., B65: 222, 224, pl. 7, figs. 1, 3.

Deep-abyssal record.-Northeastern Atlantic, ca. $61^{\circ} \mathrm{N} ., 17^{\circ} \mathrm{W}$., 2276 meters, one specimen.

Distribution.-North Atlantic, from Davis Strait (ca. $66^{\circ} \mathrm{N}$.) to ca. $37^{\circ} \mathrm{N}$. in the west and from ca. $64^{\circ} \mathrm{N}$. to the Gulf of Gascony in the east. Once found floating at the surface, depth range otherwise 155-1669 meters, with one deep-abyssal record. Rare above 500 meters, frequent in 500-1200 meters, accidental in deep-abyssal waters.

Length.-35-1000 mm.

Remarks.-There is evidence that the species may occasionally rise into intermediate waters. 


\section{Chalinura murrayi Günther}

Coryphaenoides murrayi Günther, 1878, Ann. Mag. Nat. Hist., (5), 2: 26 -Pacific off New Zealand, ca. $40^{\circ}$ S., $177^{\circ}$ E., 2012 meters.

Macrurus murrayi Günther, 1887, Rep. Sci. Res. Voy. Challenger, Zool., 22: 146, pl. 34, fig. A.

Chalinura murrayi Goode and Bean, 1895, Ocean. Ichth., p. 412.

Macrurus (Coryphaenoides) mediterraneus Holt and Byrne, 1906, Rep. Fish. Ireland 1905, Sci. Invest., 2: 25; 1910, op. cit., 1908, 5: 5.

Macrurus (Chalinura) murrayi Murray and Hjort, 1912, Depths of Ocean, p. 398; Koefoed, 1927, Rep. Sci. Res. M. Sars No. Atl. Deep-sea Exp. 1910, 4, (1), p. 103.

Coryphaenoides murrayi Farran, 1924, Proc. Roy. Irish Acad., B36: 102, pl. 7, fig. 3, text fig. 3; Fowler, 1936, Bull. Amer. Mus. Nat. Hist., 70 : 456.

Chalinura murrayi europaea Nybelin, 1948, Göteborgs K. Vetensk. Vitt.Samh. Handl., (B), 5, (16), p. 61, pl. 4, figs. 1, 2, text fig. 6.

Deep-abyssal records.-Eastern Atlantic, ca. $35^{\circ}$ N., $8^{\circ}$ W., 2150 3000 meters, fifteen specimens.

Eastern Atlantic, ca. $35^{\circ} \mathrm{N} ., 8^{\circ} \mathrm{W} ., 2055$ meters, one specimen.

Eastern Atlantic, ca. $57^{\circ} \mathrm{N}$., $11^{\circ} \mathrm{W}$., 1853 meters, two specimens.

Southwestern Pacific off New Zealand, 2012 meters, five specimens, including the type.

Distribution.-Eastern Atlantic, three deep-abyssal records and, off southwestern Ireland, ten specimens from four hauls in 12071797 meters. Southwestern Pacific, one deep-abyssal record.

Length. $-77-500+\mathrm{mm}$.

Remarks.-The species is close to C. mediterranea and the two eastern Atlantic specimens referred to that species by Roule (1919, p. 87) may prove to be murrayi. Roule's specimens were taken near the Azores in 1940 and 1886 meters.

\section{Chalinura mediterranea Giglioli}

Chalinura mediterranea Giglioli, 1893, Zool. Anz., 16: 342-Mediterranean off Sardinia.

Coryphaenoides serratus Giglioli, *1881, Atti 3 Congr. Geogr. Intern., 1: 366; 1882, Nature, London, 25: 505; *1882, Ann. Sci. Nat. Zool., 13, (9); *1883, Atti 3 Congr. Geogr. Intern., 2: 195; Giglioli and Issel, *1884, Pelagos, p. 227; Carus, 1893, Prodr. Faunae Medit., 2: 583.

Chalinura mediterranea Goode and Bean, 1895, Ocean. Ichth., p. 533, fig. $345, a$.

Coryphaenoides mediterraneus Gilbert and Hubbs, 1916, Proc. U. S. Nat. Mus., 51: 143. 
Deep-abyssal records and distribution.-Mediterranean off Sardinia, ca. $41^{\circ} \mathrm{N}$., $6^{\circ}-7^{\circ}$ E., 2904,2805 meters, two specimens.

Length.-215, $235 \mathrm{~mm}$.

Remarks.-The two specimens reported by Roule (1919, p. 87) from the eastern Atlantic should be re-examined. Other reports outside the Mediterranean have proved erroneous (Farran, 1924, p. 102; Nybelin, 1948, p. 61). See remarks under C. murrayi, above. Fowler (1936, p. 466) has placed Roule's specimens in the synonymy of Trachonurus asperrimus Vaillant, without explanation or further description.

\section{Chalinura fernandeziana Günther}

Macrurus fernandezianus Günther, 1887, Rep. Sci. Res. Voy. Challenger, Zool., 22: 145, pl. 37, fig. B-Pacific south of Juan Fernandez, ca. $33^{\circ}$ S., $78^{\circ} \mathrm{W}$.

Chalinura fernandezianus Goode and Bean, 1895, Ocean. Ichth., p. 412.

Coryphaenoides fernandezianus Gilbert and Hubbs, 1916, Proc. U. S. Nat. Mus., $51: 143$.

Deep-abyssal record and distribution.--Southeastern Pacific, 2515 meters, one specimen.

Length. $-279 \mathrm{~mm}$.

\section{Chalinura filifera Gilbert}

Chalinura filifera Gilbert, 1896, Rep. U. S. Comm. Fish, 1893: 458-Pacific off the Queen Charlotte Islands.

Chalinura filifera Jordan and Evermann, 1898, Bull. U. S. Nat. Mus., 47: 2577; Clemens and Wilby, 1946, Bull. Fish. Res. Bd. Canada, 68: 135, fig. 77.

Coryphaenoides filifer Gilbert and Hubbs, 1916, Proc. U. S. Nat. Mus., 51 : 143.

Deep-abyssal record and distribution.-Eastern Pacific, 2904 meters, three specimens.

Length. $-520-550 \mathrm{~mm}$.

\section{Chalinura liocephala Günther}

Macrurus liocephalus Günther, 1887, Rep. Sci. Res. Voy. Challenger, Zool., 22: 145, pl. 38, fig. A-Pacific off Japan, ca. $34^{\circ}$ N., $140^{\circ}$ E., 3429 meters.

Chalinura liocephala Goode and Bean, 1895, Ocean. Ichth., p. 412; Jordan and Gilbert, in Jordan and Starks, 1904, Bull. U. S. Fish Comm., 22: 608.

Coryphaenoides liocephalus Gilbert and Hubbs, 1916, Proc. U. S. Nat. Mus., $51: 143$.

Deep-abyssal records and distribution.-Mid-Pacific, ca. $36^{\circ} \mathrm{N}$., $178^{\circ}$ E., 3749 meters, two specimens. 
Western Pacific off Japan, 3429 meters, one specimen.

Length. - 190.5-419 $\mathrm{mm}$.

\section{Chalinura simula Goode and Bean}

Chalinura simula Goode and Bean, 1883, Bull. Mus. Comp. Zool., 10: 199 -Atlantic, ca. $41^{\circ}$ N., $65^{\circ}$ W., 2271 meters.

Macrurus simulus Günther, 1887, Rep. Sci. Res. Voy. Challenger, Zool., 22: 145.

Chalinura simula Goode and Bean, 1895, Ocean. Ichth., p. 412, fig. 345; Jordan and Evermann, 1898, Bull. U. S. Nat. Mus., 47 : 2578; 1900, op. cit., fig. 910; Roule, 1919, Rés. Camp. Sci. Monaco, 52: 86; Schroeder, 1940, Copeia, p. 234; Parr, 1946, Bull. Bingham Oceanogr. Coll., 10, (1), pp. 31, 65, figs. 9, 20; Nybelin, 1951, Rep. Swedish Deep-sea Exp. 1947-1948, Zool., 2, (1), p. 24.

Macrurus (Chalinura) simulus Lütken, 1898, Danish Ingolf Exp., 2, (1), p. 28; Ehrenbaum, 1902, Fauna Arct., 2: 117; Murray and Hjort, 1912, Depths of Ocean, p. 398; Koefoed, 1927, Rep. Sci. Res. M. Sars No. Atl. Deep-sea Exp. 1910, 4, (1), p. 100.

Coryphaenoides simulus Gilbert and Hubbs, 1916, Proc. U. S. Nat. Mus., 51 : 143; Fowler, 1936, Bull. Amer. Mus. Nat. Hist., $70: 457$.

Deep-abyssal records.-Mid-Atlantic west of the mid-Atlantic ridge, ca. $40^{\circ} \mathrm{N}$., $35^{\circ} \mathrm{W}$., $4590-4600$ meters, one specimen.

Mid-Atlantic on the mid-Atlantic ridge, ca. $45^{\circ} \mathrm{N}$., $25^{\circ} \mathrm{W}$., 3120 meters, two specimens.

Western Atlantic, ca. $39^{\circ} \mathrm{N}$., $66^{\circ} \mathrm{W}$., 3704 meters, one specimen.

Western Atlantic, ca. $38^{\circ} \mathrm{N} ., 69^{\circ} \mathrm{W}$., 3166 meters, (?) specimens.

Western Atlantic, $37^{\circ}-41^{\circ}$ N., $73^{\circ}-65^{\circ}$ W., 2552, 2455, 2271 meters, an unstated number of specimens from three hauls.

Denmark Strait, ca. $64^{\circ} \mathrm{N}$., $31^{\circ} \mathrm{W}$., 2261 meters, two specimens.

Denmark Strait, ca. $61^{\circ} \mathrm{N}$., $30^{\circ} \mathrm{W}$., 2075 meters, four specimens.

Eastern Atlantic, ca. $34^{\circ}$ N., $33^{\circ}$ W., 2615-2865 meters, five specimens in one haul.

Eastern Atlantic, $37^{\circ}-38^{\circ}$ N., $26^{\circ}-28^{\circ}$ W., 2102, 1919 meters, two specimens.

Distribution.-North Atlantic from Denmark Strait (ca. $65^{\circ} \mathrm{N}$.) to ca. $34^{\circ} \mathrm{N}$. in the east, ca. $40^{\circ} \mathrm{N}$. centrally and ca. $31^{\circ} \mathrm{N}$. in the west, twelve deep-abyssal records, two specimens in 1669 meters and three juvenile specimens, about $25 \mathrm{~mm}$. long, doubtfully referred to this species, in 610 and 1183 meters. A deep-abyssal species with two specimens known from the abyssal plain.

Length.-25(?) and 160-620 $\mathrm{mm}$. 
Chalinura carapina Goode and Bean. Table 17.

Coryphaenoides carapinus Goode and Bean, 1883, Bull. Mus. Comp. Zool., 10: 197-Atlantic off United States coast, figured specimen from Blake Station $342,39^{\circ} 43^{\prime}$ N., $70^{\circ} 55^{\prime} 25^{\prime \prime}$ W., 1833 meters.

Coryphaenoides carapinus Günther, 1887, Rep. Sci. Res. Voy. Challenger, Zool., 22: 138; Goode and Bean, 1895, Ocean. Ichth., p. 404, fig. 339; Jordan and Evermann, 1898, Bull. U. S. Nat. Mus., 47: 2579; 1900, op. cit., fig. 911; Roule and Angel, 1933, Rés. Camp. Sci. Monaco, 86: 62, pl. 3, fig. 29; Gregory, 1933, Trans. Amer. Phil. Soc., 23, (2), p. 383, fig. 262; Fowler, 1936, Bull. Amer. Mus. Nat. Hist., 70 : 1246, fig. 535; Schroeder, 1940, Copeia, p. 235.

Macrurus carapinus Koefoed, 1927, Rep. Sci. Res. M. Sars No. Atl. Deep-sea Exp. 1910, 4, (1), pp. 106, 108.

Chalinura carapina Parr, 1946, Bull. Bingham Oceanogr. Coll., 10, (1), pp. 31, 68, figs. 9, 21; Nybelin, 1951, Rep. Swedish Deep-sea Exp. 1947-1948, Zool., 2, (1), pp. 14, 16.

Distribution.-Eastern Atlantic, eight specimens from deepabyssal waters (ca. $34^{\circ} \mathrm{N}$., $33^{\circ} \mathrm{W}$.; ca. $27^{\circ} \mathrm{N} ., 14^{\circ} \mathrm{W}$.; and ca. $22^{\circ}$ N., $23^{\circ} \mathrm{W}$.) and one specimen off the Azores in 1740 meters. MidAtlantic on the mid-Atlantic ridge (ca. $45^{\circ} \mathrm{N}$., $25^{\circ} \mathrm{W}$.) and east of it (ca. $1^{\circ} \mathrm{N} ., 18^{\circ} \mathrm{W}$.), two deep-abyssal records. Western Atlantic, $35^{\circ}-42^{\circ} \mathrm{N}$., twenty-five deep-abyssal records and ten hauls in $1211-$ 1748 meters. Depth range 1211-5300 meters. Chiefly deep-abyssal, with two specimens known from the abyssal plain.

Length. $-120-340 \mathrm{~mm}$.

\section{Chalinura ferrieri Regan}

Chalinura ferrieri Regan, 1913, Trans. Roy. Soc. Edinburgh, 49: 236, pl. 2, fig. 1-Antarctic, ca. $71^{\circ} \mathrm{S} ., 16^{\circ} \mathrm{W} ., 2579$ meters.

Chalinura ferrieri Waite, 1916, Sci. Rep. Austr. Antarctic Exp. 1911-1914, (C), 3, (1), p. 13, fig. 1.

Coryphaenoides ferrieri Gilbert and Hubbs, 1916, Proc. U. S. Nat. Mus., 51: 143.

Deep-abyssal records and distribution.-Antarctic, ca. $64^{\circ}$ S., $127^{\circ}$ E., 3109 meters, one specimen.

Antarctic off Coats Land, 2579 meters, one specimen.

Length. $-230-250 \mathrm{~mm}$.

\section{Chalinura whitsoni Regan}

Chalinura whitsoni Regan, 1913, Trans. Roy. Soc. Edinburgh, 49: 236, pl. 2, fig. 2-Antaretic, ca. $71^{\circ}$ S., $16^{\circ}$ W., 2579 meters.

Chalinura whitsoni Waite, 1916, Sci. Rep. Austr. Antarctic Exp. 1911-1914, (C), 3, (1), p. 14. 
Coryphaenoides whitsoni Gilbert and Hubbs, 1916, Proc. U. S. Nat. Mus., 51 : 143; Norman, 1937, Rep. B.A.N.Z. Antarctic Res. Exp. 1929-1931, (B), 1, (2), p. 68 .

Deep-abyssal records.-South Atlantic, ca. $48^{\circ}$ S., $10^{\circ}$ W., 3185 meters, one specimen.

Antarctic off Coats Land, 2579 meters, one specimen.

Distribution.-Antarctic and South Atlantic, five specimens known, two of them deep-abyssal, two in 1590 meters (ca. $63^{\circ} \mathrm{S}$., $101^{\circ} \mathrm{E}$.) and one in 604 meters (ca. $60^{\circ} \mathrm{S} ., 58^{\circ} \mathrm{E}$.).

Length. $-270-525 \mathrm{~mm}$. (572 $\mathrm{mm}$. fresh).

\section{Chalinura guentheri Vaillant. Table 18.}

Macrurus Guentheri Vaillant, 1888, Exp. Sci. Trav. Talis., Poiss., p. 386 -Atlantic off Morocco, 2200 or 2115 meters (type locality not stated; see footnote, p. 110).

Macrurus sclerorhynchus Günther, 1887, Rep. Sci. Res. Voy. Challenger, Zool, 22: 133, pl. 32, fig. A; Brauer, 1906, Wiss. Ergebn. Deutschen Tiefsee Exp. Valdivia, 15, (1), p. 265.

Macrurus sclerorhynchus var. Vaillant, 1888, Exp. Sci. Trav. Talis., Poiss., p. 54.

Macrurus holotrachys Vaillant, 1888, op. cit., p. 241, pl. 22, fig. 3.

Macrurus guntheri Collett, 1896, Rés. Camp. Sci. Monaco, 10: 80, pl. 3, fig. 10; Holt and Byrne, 1906, Rep. Fish. Ireland 1905, Sci. Invest., 2: 25; Murray and Hjort, 1912, Depths of Ocean, p. 397; Roule, 1919, Rés. Camp. Sci. Monaco, 52: 79; Vaillant, in Roule, 1919, op. cit., p. 134; Koefoed, 1927, Rep. Sci. Res. M. Sars No. Atl. Deep-sea Exp. 1910, 4, (1), p. 87 , figs. 23,24 .

Coryphaenoides (Macrurus) guntheri Collett, 1905, Rep. Norwegian Fish. Mar. Invest., 2, (3), p. 56.

Coryphaenoides guentheri Gilbert and Hubbs, 1916, Proc. U. S. Nat. Mus., 51: 144 .

Lionurus Guntheri Farran, 1924, Proc. Roy. Irish Acad., B36: 116, fig. 7. Macruroplus guentheri Fowler, 1936, Bull. Amer. Mus. Nat. Hist., 70 : 463.

Chalinura guntheri Nybelin, 1948, Göteborgs K. Vetensk. Vitt.-Samh. Handl., (B), 5, (16), p. 65 , fig. 6 .

Deep-abyssal records.-Eastern Atlantic, ca. $27^{\circ}$ N., $14^{\circ}$ W., 2603 meters, one specimen.

Eastern Atlantic southeast of Portugal and off the coast of Morocco, 1994-2300 meters, thirteen specimens from five hauls.

Eastern Atlantic from the Azores to ca. $57^{\circ}$ N., $11^{\circ}$ W., 18461940 meters, seventy-three specimens from five hauls.

(?)Indian Ocean, Gulf of Aden, 1840 meters, one specimen. 
Distribution.-Eastern Atlantic from the Faroe-Shetland Channel to off the Canary Islands, eleven deep-abyssal records and thirty-four specimens from five hauls in 1200-1797 meters. (?)North Indian Ocean, one deep-abyssal record. Probably not rare in the upper part of the deep-abyssal zone. Center of distribution perhaps in $1800-2000$ meters.

Length. $-136-480 \mathrm{~mm}$.

Remarks.-The Indian Ocean record is based on a specimen criginally identified as $M$. sclerorhynchus (Brauer, 1906, p. 265) and referred to guentheri by Koefoed (1927, p. 87). Nybelin (1948, p. 65) doubted that Koefoed's conclusion was correct.

\section{Chalinura serrula Bean}

Chalinura serrula Bean, 1890, Proc. U. S. Nat. Mus., 13: 37--Pacific off Prince of Wales Island, ca. $55^{\circ}$ N., $136^{\circ} \mathrm{W}$.

Chalinura serrula Goode and Bean, 1895, Ocean. Ichth., p. 412; Jordan and Evermann, 1898, Bull. U. S. Nat. Mus., 47: 2576.

Coryphaenoides serrulus Gilbert and Hubbs, 1916, Proc. U. S. Nat. Mus., 51 : 144.

Deep-abyssal record and distribution.--Northeastern Pacific, 2869 meters, three specimens.

Length.-?--318 mm.

\section{Chalinura brevibarbis Goode and Bean}

Chalinura brevibarbis Goode and Bean, 1895, Ocean. Ichth., p. 413-Atlantic, ca. $41^{\circ} \mathrm{N} ., 66^{\circ} \mathrm{W} ., 2295$ meters.

Macrurus (Chalinura) brevibarbis Murray and Hjort, 1912, Depths of Ocean, p. 398; Koefoed, 1927, Rep. Sci. Res. M. Sars No. Atl. Deep-sea Exp. 1910, 4, (1), p. 105, pl. 5, fig. 10, text figs. 36, 37.

Coryphaenoides brevibarbus Gilbert and Hubbs, 1916, Proc. U. S. Nat. Mus., $51: 143$.

Chalinura brevibarbis Parr, 1946, Bull. Bingham Oceanogr. Coll., 10, (1), pp. 31, 71, figs. 9,22 .

Deep-abyssal records.-Eastern Atlantic, ca. $45^{\circ}$ N., $9^{\circ}$ W., 4700 meters, one specimen.

Eastern Atlantic, ca. $57^{\circ}$ N., $11^{\circ}$ W., 1853 meters, one specimen.

Mid-Atlantic on the mid-Atlantic ridge, ca. $45^{\circ} \mathrm{N}$., $25^{\circ} \mathrm{W}$, 3120 meters, three specimens.

Western Atlantic, $38^{\circ}-41^{\circ}$ N., $66^{\circ}-70^{\circ}$ W., 3166, 2491, 2295 meters, an unstated number of specimens from three hauls.

Distribution.-Eastern and mid-Atlantic, three deep-abyssal records. Western Atlantic, $38^{\circ}-41^{\circ} \mathrm{N}$., $66^{\circ}-71^{\circ} \mathrm{W}$., an unstated 
number of specimens from three deep-abyssal hauls and two in 1813 and 1748 meters. Probably chiefly deep-abyssal.

Length. - 240-345 mm.

\section{Macrourus paradoxus Smith and Radcliffe}

Macrourus paradoxus Smith and Radcliffe, in Radcliffe, 1912, Proc. U. S. Nat. Mus., 43: 115, pl. 25, fig. 1-Pacific off Palawan, Philippine Islands.

Coryphaenoides paradoxus Gilbert and Hubbs, 1916, Proc. U. S. Nat. Mus., 51: 143.

Deep-abyssal record and distribution.-Western Pacific, 2021 meters, one specimen.

Length. $-585 \mathrm{~mm}$.

\section{Macrourus bucephalus Garman}

Macrurus bucephalus Garman; 1899, Mem. Mus. Comp. Zool., 24: 195, pl. 44, fig. 2-Pacific off Mexico, Panama, and Ecuador (type locality not designated).

Coryphaenoides bucephalus Gilbert and Hubbs, 1916, Proc. U. S. Nat. Mus., 51 : 143.

Deep-abyssal records.-Eastern Pacific, ca. $1^{\circ}$ N., $80^{\circ}$ W., 2877 meters, (?) specimens.

Eastern Pacific, ca. $7^{\circ}$ N., $79^{\circ}$ W., 1865 meters, (?) specimens.

Distribution.-Eastern Pacific, ca. $1^{\circ}-7^{\circ}$ N., $79^{\circ}-86^{\circ}$ W. and ca. $23^{\circ} \mathrm{N}$., $107^{\circ} \mathrm{W}$., two deep-abyssal records and an unstated number of specimens from two hauls in 1599 and 245 meters.

Length.-Not known.

Remarks.-Since Garman did not include lengths in his type description it is not possible to determine the age of the specimen taken in 245 meters.

\section{Macrourus carminifer Garman}

Macrurus carminifer Garman, 1899, Mem. Mus. Comp. Zool., 24: 204, pl. 46, fig. 2-Pacific off Panama, ca. $7^{\circ}$ N., $78^{\circ}-80^{\circ}$ W., (type locality not designated).

Coryphaenoides carminatus Gilbert and Hubbs, 1916, Proc. U. S. Nat. Mus., 51: 144.

Deep-abyssal record.-Eastern Pacific, ca. $7^{\circ}$ N., $79^{\circ}$ W., 1865 meters, (?) specimens.

Distribution.-Eastern Pacific, ca. $7^{\circ} \mathrm{N}$., $78^{\circ}-80^{\circ} \mathrm{W}$., an unstated number of specimens from four hauls, one deep-abyssal, the others in 1335,1271 and 588 meters.

Length.-?-305 $\mathrm{mm}$. 


\section{Macrourus anguliceps Garman}

Macrurus anguliceps Garman, 1899, Mem. Mus. Comp. Zool., 24: 212, 363, pl. G, fig. 1, pls. 50, 83, fig. 2-Pacific off northern South America, off

Panama, and in the Gulf of California (type locality not designated).

Coryphaenoides anguliceps Gilbert and Hubbs, 1916, Proc. U. S. Nat. Mus., 51: 144 .

Deep-abyssal records.-Eastern Pacific from ca. $0^{\circ} 36^{\prime} \mathrm{S} ., 86^{\circ} \mathrm{W}$. to ca. $7^{\circ} \mathrm{N} ., 79^{\circ} \mathrm{W} ., 2417,2322,2150,2070,1951$ meters, an unstated number of specimens from five stations.

Distribution.-Eastern Pacific off northern South America and Panama, and in the Gulf of California, ca. $26^{\circ} \mathrm{N} ., 110^{\circ} \mathrm{W}$., five deep-abyssal records and three hauls in 1271-1570 meters.

Length._-?-343 $\mathrm{mm}$.

\section{Macrourus longicirrhus Gilbert}

Macrourus longicirrhus Gilbert, 1905, Bull. U. S. Fish Comm., 23: 672, fig. 263-Pacific off Hawaii.

Coryphaenoides longicirrhus Gilbert and Hubbs, 1916, Proc. U. S. Nat. Mus., 51 : 144; Fowler, 1938, Monogr. Acad. Nat. Sci. Philadelphia, 2: 217.

Deep-abyssal record.-Hawaiian Islands, 1829-2403 meters, one specimen.

Distribution.-Hawaiian Islands, one deep-abyssal record and one specimen from an unknown depth.

Length. $-590-965 \mathrm{~mm}$.

\section{Macrourus aequatoris Smith and Radcliffe}

Macrourus aequatoris Smith and Radcliffe, in Radcliffe, 1912, Proc. U. S. Nat. Mus., 43: 120, pl. 26, fig. 3-Gulf of Tomini, Celebes, 1992 meters.

Coryphaenoides aequatoris Gilbert and Hubbs, 1920, Bull. U. S. Nat. Mus., 100, (1), p. 419; Weber and de Beaufort, 1929, Fishes Indo-Austr. Arch., 5: 30 .

Deep-abyssal records and distribution.-Western Pacific, Gulf of Tomini, 1997, 1992 meters, two specimens.

Length. $-166+-188 \mathrm{~mm}$.

\section{Macrourus cinereus Gilbert}

Macrurus cinereus Gilbert, 1896, Rep. U. S. Comm. Fish, 1893: 457-Bering Sea, ca. $55^{\circ} \mathrm{N} ., 155^{\circ}$ W., 1271 meters.

Macrurus cinereus Jordan and Evermann, 1898, Bull. U. S. Nat. Mus., 47: 2586; Jordan and Gilbert, 1899, Rep. Fur-seal Invest., 3: 487; Evermann and Goldsborough, 1907, Bull. U. S. Bur. Fish., 26: 350; Gilbert and Burke, 1912, op. cit., 30: 92; Soldatov and Lindberg, 1930, Bull. Pac. Sci. Inst. Fish. Oceanogr., 5: 521; Böhlke, 1953, Stanf. Ichth. Bull., 5: 58. 
Coryphaenoides cinereus Gilbert and Hubbs, 1916, Proc. U. S. Nat. Mus., 51 : 167; 1920, Bull. U. S. Nat. Mus., 100, (1), p. 371; Schmidt, 1950, Akad. Sci. U.S.S.R., Trans. Pac. Comm., 6: 61.

Deep-abyssal record.-Bering Sea, ca. $53^{\circ}$ N., $170^{\circ}$ W., 1890 meters, (?) specimens.

Distribution.-North Pacific in the Okhotsk and Bering seas, abundant between 630 and 1400 meters, also known from one deepabyssal record and from one specimen each in 1687 and 1643 meters. Although its lower limit is below 1829 meters the species is certainly not to be considered deep-abyssal.

Length.-190.5-444.5 mm.

\section{Macrourus altipinnis Günther}

Coryphaenoides altipinnis Günther, 1877, Ann. Mag. Nat. Hist., (4), 20 : 439 -Pacific off Japan, ca. $34^{\circ}$ N., $140^{\circ}$ E., 3429 meters.

Macrurus altipinnis Günther, 1887, Rep. Sci. Res. Voy. Challenger, Zool., 22: 138, pl. 39, fig. A; Goode and Bean, 1895, Ocean. Ichth., p. 402; Jordan and Gilbert, in Jordan and Starks, 1904, Bull. U. S. Fish Comm., 22: 608 .

Coryphaenoides altipinnis Gilbert and Hubbs, 1916, Proc. U. S. Nat. Mus., 51: 144.

Deep-abyssal record.-Western Pacific off Japan, 3429 meters, the type.

Distribution.-Western Pacific off Japan, one deep-abyssal record and two specimens in 1034 meters (ca. $34^{\circ}$ N., $138^{\circ}$ E.).

Length. $-152-483 \mathrm{~mm}$.

\section{Macrourus ingolfi Lütken}

Macrurus ingolfi Lütken, 1898, Danish Ingolf Exp., 2, (1), p. 27-Atlantic south of Iceland, ca. $62^{\circ} \mathrm{N} ., 21^{\circ}$ W., 1545 meters.

Macrurus ingolfi Ehrenbaum, 1902, Fauna Arct., 2: 117.

Coryphaenoides ingolfi Gilbert and Hubbs, 1916, Proc. U. S. Nat. Mus., 51 : 144.

Deep-abyssal records.-North Atlantic in Denmark Strait, ca. $64^{\circ} \mathrm{N} ., 31^{\circ} \mathrm{W} ., 2378$ meters, one specimen.

North Atlantic, ca. $61^{\circ} \mathrm{N}$., $30^{\circ} \mathrm{W} ., 2075$ meters, one specimen.

North Atlantic, ca. $62^{\circ} \mathrm{N} ., 19^{\circ}$ W., 1904 meters, one specimen.

Distribution.-North Atlantic, $61^{\circ}-64^{\circ}$ N., $19^{\circ}-31^{\circ}$ W., three deep-abyssal specimens, one in 1669 meters and two in 1545 meters.

Length.-270-340 mm. 


\section{Macrourus sublaevis Vaillant}

Coryphaenoides sublaevis Vaillant, 1888, Exp. Sci. Trav. Talis., Poiss., p. 386 -Talisman Station 111, Cape Verde Islands, 580 meters (see footnote, p. 110).

Coryphaenoides aequalis Vaillant, 1888, op. cit., p. 225 (part), pl. 19, fig. 2. Malacocephalus sublaevis Goode and Bean, 1895, Ocean. Ichth., p. 535.

Coryphaenoides sublaevis Gilbert and Hubbs, 1916, Proc. U. S. Nat. Mus., $51: 144$.

Distribution.-Eastern Atlantic.

Remarks.--There is some confusion attending Vaillant's records of this species. He described as Coryphaenoides aequalis fifty-two specimens taken by the Talisman off Morocco, Soudan, the Cape Verde Islands and on Arguin Banc, in 140-2200 meters. In the appendix of the same volume the author stated that the specimens represented a new species, Coryphaenoides sublaevis, except for certain individuals with different dentition. These, according to Vaillant, were actually Malacocephalus laevis Lowe. Professor Bertin has written (1950, in litt.) that the Museum of Natural History in Paris now has only eight of the original fifty-two specimens, including the type of $C$. sublaevis, from Station 111, Cape Verde Islands, 580 meters (the specimen for which measurements were given by Vaillant on page 227). Bertin also wrote that Vaillant did not mark the specimens he had separated as M. laevis. The latter species has been reported only from shallower strata and the deeper records listed by Vaillant perhaps refer to sublaevis. There are three of these, one specimen in 2200 meters, one in 2115 meters, and one in 2104 meters.

Length.-Type $430 \mathrm{~mm}$.

\section{Macrourus hexti Alcock}

Macrurus Hextii Alcock, 1890, Ann. Mag. Nat. Hist., (6), 6: 299-Arabian Sea, ca. $11^{\circ}$ N., $74^{\circ}$ E., 1829 meters.

Macrurus hextii Alcock, 1891, Ann. Mag. Nat. Hist., (6), 7: 11; 1892, op. cit., (6), 10: 351; 1894, Jour. Asiat. Soc. Bengal, 63: 126; 1894, Ill. Zool. Investigator, Fishes, pl. 12, fig. 3; Goode and Bean, 1895, Ocean. Ichth., p. 390.

Macrurus (Macrurus) hextii Alcock, 1899, Descr. Cat. Indian Deep-sea Fishes, p. 113.

Coryphaenoides hextii Gilbert and Hubbs, 1916, Proc. U. S. Nat. Mus., 51 : 144.

Deep-abyssal record.-Arabian Sea, 1829 meters, the type.

Distribution.-Arabian Sea, one deep-abyssal specimen and one in ca. $12^{\circ} \mathrm{N}$., $71^{\circ} \mathrm{E}$., $1582-1609$ meters.

Length.-?-ca. $584 \mathrm{~mm}$. 


\section{Macrourus wood-masoni Alcock}

Macrurus Wood-Masoni Alcock, 1890, Ann. Mag. Nat. Hist., (6), 6: 301 -Arabian Sea, ca. $11^{\circ}$ N., $74^{\circ}$ E., 1829 meters.

Macrurus wood-masoni Alcock, 1891, op. cit., (6), 7 : 11; 1891, op. cit., (6), 8: $121 ; 1892$, op. cit., (6), $10: 353 ; 1894$, Ill. Zool. Investigator, Fishes, pl. 13, fig. 1; 1894, Jour. Asiat. Soc. Bengal, 63: 126; Goode and Bean, 1895, Ocean. Ichth., p. 390.

Macrurus (Macrurus) wood-masoni Alcock, 1899, Descr. Cat. Indian Deep-sea Fishes, p. 114; Brauer, 1906, Wiss. Ergebn. Deutschen Tiefsee Exp. Valdivia, 15, (1), p. 26.

Coryphaenoides wood-Masoni Gilbert and Hubbs, 1916, Proc. U. S. Nat. Mus., $51: 144$.

Deep-abyssal records.-Gulf of Aden, 1840 meters, one specimen. Arabian Sea, 1829 meters, the type.

Distribution.-North Indian Ocean off northeast Africa (ca. $1^{\circ} \mathrm{S}$., $41^{\circ} \mathrm{E}$.), in the Gulf of Aden, and in the Arabian Sea $\left(6^{\circ}-15^{\circ} \mathrm{N}\right.$., $72^{\circ}-$ $78^{\circ} \mathrm{E}$.), two deep-abyssal specimens and an unknown number in $693,1022,1350$ and 1650 meters.

Length. $-340-495 \mathrm{~mm}$.

\section{Macrourus hoskyni Alcock}

Macrurus Hoskynii Alcock, 1890, Ann. Mag. Nat. Hist., (6), 6: 214-Bay of Bengal, ca. $18^{\circ} \mathrm{N} ., 85^{\circ} \mathrm{E}$.

Macrurus hoskynii Alcock, 1891, op. cit., (6), 7: 11; 1894, Ill. Zool. Investigator, Fishes, pl. 9, fig. 4; 1894, Jour. Asiat. Soc. Bengal, 63: 126; Goode and Bean, 1895, Ocean. Ichth., p. 390.

Macrurus (Macrurus) hoskynii Alcock, 1899, Descr. Cat. Indian Deep-sea Fishes, p. 116.

Coryphaenoides hoskynii Gilbert and Hubbs, 1916, Proc. U. S. Nat. Mus., $51: 144$.

Deep-abyssal record and distribution.-Bay of Bengal, 2395 meters, one specimen.

Length. - $362 \mathrm{~mm}$.

\section{Macrourus camurus Smith and Radcliffe}

Macrourus camurus Smith and Radcliffe, in Radcliffe, 1912, Proc. U. S. Nat. Mus., 43: 122, pl. 27, fig. 2-Pacific off Palawan, Philippine Islands.

Coryphaenoides camurus Gilbert and Hubbs, 1916, Proc. U. S. Nat. Mus., 51 : 144 ; 1920, Bull. U. S. Nat. Mus., 100, (1), p. 421.

Deep-abyssal record and distribution.-Western Pacific, 2021 meters, one specimen.

Length. $-102 \mathrm{~mm}$. 


\section{Macrourus orthogrammus Smith and Radcliffe}

Macrourus orthogrammus Smith and Radcliffe, in Radcliffe, 1912, Proc. U. S. Nat. Mus., 43: 123, pl. 27, fig. 3-Pacific near Gomono Island, Dutch East Indies.

Coryphaenoides orthogrammus Gilbert and Hubbs, 1916, Proc. U. S. Nat. Mus., 51 : 144; 1920, Bull. U. S. Nat. Mus., 100, (1), p. 421; Weber and de Beaufort, 1929, Fishes Indo-Austr. Arch., 5: 32.

Deep-abyssal record and distribution.-Western Pacific, 2308 meters, one specimen.

Length.-180 $\mathrm{mm}$.

\section{Macrourus asper Günther}

Coryphaenoides asper Günther, 1877, Ann. Mag. Nat. Hist., (4), 20: 440 -Pacific south of Japan, ca. $34^{\circ}$ N., $140^{\circ}$ E.

Macrurus asper Günther, 1887, Rep. Sci. Res. Voy. Challenger, Zool., 22 : 137, pl. 36, fig. A.

Coryphaenoides asper Gilbert and Hubbs, 1916, Proc. U. S. Nat. Mus., 51 : 144.

Deep-abyssal record and distribution.-Western Pacific, 3429 meters, one specimen.

Length. $-330 \mathrm{~mm}$.

\section{Macrourus albatrossus Townsend and Nichols}

Macrourus albatrossus Townsend and Nichols, 1925, Bull. Amer. Mus. Nat. Hist., 52:17, pl. 4-Pacific off Lower California, ca. $31^{\circ}$ N., $118^{\circ} \mathrm{W}$.

Deep-abyssal record and distribution.-Eastern Pacific, 1968 meters, one specimen.

Length.-Not stated.

\section{Hemimacrurus acrolepis Bean}

Macrurus acrolepis Bean, 1884, Proc. U. S. Nat. Mus., 6: 362-Pacific off Washington, from the stomach of a seal.

Macrurus acrolepis Günther, 1887, Rep. Sci. Res. Voy. Challenger, Zool., 22: 124; Gilbert, 1896, Rep. U. S. Comm. Fish, 1893: 457; 1899, op. cit., 1898: 28; 1915, Proc. U. S. Nat. Mus., 48: 376; Jordan and Evermann, 1898, Bull. U. S. Nat. Mus., 47: 2585; Jordan and Gilbert, 1899, Rep. Fur-seal Invest., 3: 487, pl. 82; Evermann and Goldsborough, 1907, Bull. U. S. Bur. Fish., 26: 350, fig. 131; Gilbert and Burke, 1912, op. cit., 30: 91; Gilbert and Hubbs, 1916, Proc. U. S. Nat. Mus., 51: 162; Townsend and Nichols, 1925, Bull. Amer. Mus. Nat. Hist., 52: 16, pl. 4; Johnsen, 1927, Nyt Mag. Naturv., B65: 241; Taranetz, 1937, Bull. Pac. Sci. Inst. Fish. Oceanogr., 11: 170; Clemens and Wilby, 1946, Bull. Fish. Res. Bd. Canada, 68: 136, fig. 78. 
Coryphaenoides bona-nox Jordan and Thompson, 1914, Mem. Carnegie Mus., 6, (4), p. 305 , pl. 38.

Nematonurus bona-nox Gilbert and Hubbs, 1916, Proc. U. S. Nat. Mus., $51: 162$.

Hemimacrurus acrolepis Fraser-Brunner, 1935, Proc. Roy. Irish Acad., B42: 322 .

Deep-abyssal records.-Bering Sea, ca. $53^{\circ}$ N., $171^{\circ}$ W., 2226 meters, (?) specimens.

Eastern Pacific off the American coast from ca. $29^{\circ} \mathrm{N}$. to the vicinity of San Diego, 2182, 1994, 1968, 1937 meters, an unstated number of specimens from four hauls.

Eastern Pacific near San Diego, 2469-1235 meters, (?) specimens, perhaps caught in deep-abyssal waters.

Distribution.-Pacific from ca. $54^{\circ}$ N., $176^{\circ}$ E. in Bering Sea to off southern California, six deep-abyssal records; once taken from the stomach of a seal, once at a depth of 155 meters (two specimens) north of the Aleutian Islands, otherwise in 605-1437 meters, at which depths it is abundant. Western Pacific, Sagami Sea, Japan, three specimens, depth of capture unknown. Perhaps partially deep-abyssal.

Length. $-127-780 \mathrm{~mm}$.

\section{Grenurus hirundo Collett}

Macrurus hirundo Collett, 1896, Rés. Camp. Sci. Monaco, 10: 72, pl. 2, fig. 8-Atlantic off the Azores, ca. $38^{\circ}$ N., 30 ${ }^{\circ}$ W., 1266 meters.

Macrurus hirundo Roule, 1919, Rés. Camp. Sci. Monaco, 52: 80; Belloc, 1949, Bull. Inst. Océanogr. Monaco, 958: 17.

Macruroplus hirundo Fowler, 1936, Bull. Amer. Mus. Nat. Hist., 70 : 465.

Grenurus hirundo Nybelin, 1948, Göteborgs K. Vetensk. Vitt.-Samh. Handl., (B), 5, (16), p. 67, pl. 4, fig. 3 .

Deep-abyssal records.--Eastern Atlantic, ca. $35^{\circ}$ N., $8^{\circ}$ W., 2150 2300 meters, one specimen.

Eastern Atlantic, ca. $39^{\circ}$ N., $29^{\circ}$ W., 1900 meters, five specimens.

Distribution.-Eastern Atlantic only, from off the Azores to the Cape Verde Islands (ca. $39^{\circ}-15^{\circ} \mathrm{N}$.), two deep-abyssal records and ten specimens in 1266-1550 meters.

Length. $-100-208 \mathrm{~mm}$.

\section{Grenurus flagellicauda Koefoed}

Macrurus flagellicauda Koefoed, 1927, Rep. Sci. Res. M. Sars No. Atl. Deepsea Exp. 1910, 4, (1), p. 99, pl. 5, fig. 8, text figs. 34, 35-Atlantic, ca. $45^{\circ} \mathrm{N} ., 25^{\circ}$ W., 3120 meters. 
Grenurus flagellicauda Nybelin, 1948, Göteborgs K. Vetensk. Vitt.-Samh. Handl., (B), 5, (16), p. 69, pl. 4, fig. 4.

Deep-abyssal records and distribution.-Mid-Atlantic on the midAtlantic ridge, ca. $45^{\circ} \mathrm{N}$., $25^{\circ} \mathrm{W} ., 3120$ meters, four specimens.

Eastern Atlantic, ca. $35^{\circ}$ N., $8^{\circ}$ W., 2150-2300 meters, one specimen.

Length. $-95+\mathrm{mm}$. and ca. 300-400 mm.

\section{Oxygadus labiatus Koehler}

Macrurus labiatus Koehler, 1896, Ann. Univ. Lyon, 26: 497, pl. 27, figs. 7, 8-Gulf of Gascony, 570-700 meters.

Macrurus japonicus Vaillant, 1888, Exp. Sci. Trav. Talis., Poiss., pp. 254, 386, pl. 21, fig. 1.

Coryphaenoides (Coelorhynchus) talismani Collett, 1905, Rep. Norwegian Fish. Mar. Invest., 2, (3), p. 58.

Macrurus labiatus Holt and Byrne, 1906, Rep. Fish. Ireland 1905, Sci. Invest., 2: 25; Farran, 1924, Proc. Roy. Irish Acad., B36: 109, pl. 7, fig. 6, text fig. 5 .

Macrurus (Coelorhynchus) talismani Murray and Hjort, 1912, Depths of Ocean, p. 397; Koefoed, 1927, Rep. Sci. Res. M. Sars No. Atl. Deep-sea Exp. 1910, 4, (1), p. 78.

Coelorhynchus vaillanti Roule, 1916, Bull. Inst. Océanogr. Monaco, 320: 20; 1919, Rés. Camp. Sci. Monaco, 52: 81, pl. 3, fig. 3; Belloc, 1949, Bull. Inst. Océanogr. Monaco, 958: 16.

Coelorhynchus talismani Gilbert and Hubbs, 1916, Proc. U. S. Nat. Mus., $51: 145$.

Deep-abyssal record.-Eastern Atlantic off the Azores, 2220 meters, one specimen.

Distribution.-Eastern Atlantic from Faroe Channel to the Cape Verde Islands and west to the Azores, one deep-abyssal record, otherwise in 460-1710 meters. Relatively common down to about 1300 meters, probably accidental in deep-abyssal waters.

Length. $-100-460 \mathrm{~mm}$.

Remarks.-Farran (1924, p. 109) reported 132 specimens taken by the Helga in 855-1634 meters off southwestern Ireland, but he stated that figures on the average number of specimens per haul indicated that the dredging operations did not reach the lower limits of the species' normal range. This is an interesting species in that it is one of a group that has a cosmopolitan distribution, at no great depth, in tropical and temperate latitudes. The species in this group are closely related and have been sometimes confused with one another. None of the other species has ever been reported from below 1829 meters. 


\section{Hymenocephalus italicus Gigioli}

Hymenocephalus italicus Giglioli, 1882, Nature, London, 27: 198-Mediterranean off Sicily and Sardinia, 508 and 823 meters.

Malacocephalus laevis Moreau, 1881, Hist. Nat. Poiss. France, 3 : 284, fig. 183; Giglioli, 1882, Nature, London, 25: 505; Ariolà, 1904, Ann. Mus. Civ. Stor. Nat. Genova, (3), 1 : 161.

Hymenocephalus italicus Giglioli and Issel, ${ }^{*} 1884$, Pelagos, p. 228, fig.; Vaillant, 1888, Exp. Sci. Trav. Talis., Poiss., pp. 31, 211, pl. 19, fig. 1; Vaillant, in Roule, 1919, Rés. Camp. Sci. Monaco, 52: 134; Goode and Bean, 1895, Ocean. Ichth., p. 406; Lo Bianco, 1909, Mitt. Zool. Stat. Neapel, 19: 720; Roule, 1919, Rés. Camp. Sci. Monaco, 52: 86; Issel, *1932, Boll. Zool. Napoli, A3, (1), p. 15; Vinciguerra, 1932, Ann. Mus. Civ. Stor. Nat. Genova, 56: 14, pl. 1; Sanzo, 1933, Monogr. Fauna Flora Golfo Napoli, 38: 263, pl. 16, fig. 12; Brunelli and Bini, 1934, Boll. Pesc. Pisc. Idrobiol., 10, (6), p. 9, pl., fig. 9; Nobre, 1935, Fauna Marinha Portugal, 1: 164; Fowler, 1936,.Bull. Amer. Mus. Nat. Hist., 70: 460, fig. 224; Trotti, 1936, Ann. Mus. Civ. Stor. Nat. Genova, 59: 160, pls. 6-9; 1947, op. cit., 63: 43; Soljan, 1948, Fauna Flora Adriatica, 1: 176, 363, fig.; Tortonese and Trotti, 1949, Atti Accad. Ligure Sci. Lett., 6, (1), p. 60; Karlovac, 1953, Bilj. Inst. Oceanogr. Rib. Split, 4: 1; Poll, 1953, Rés. Sci. Exp. Océanogr. Belge (1948-1949), 4, (2), (3), p. 245, fig. 99.

Macrurus (Mystaconurus) italicus Günther, 1887, Rep. Sci. Res. Voy. Challenger, Zool., 22: 140.

Macrurus italicus Collett, 1896, Rés. Camp. Sci. Monaco, 10: 85, pl. 2, fig. 7; Zugmayer, 1911, op. cit., $35: 127$.

Deep-abyssal records.-Eastern Atlantic, ca. $37^{\circ}$ N., $10^{\circ} \mathrm{W}$. , 4900 meters, one specimen caught in a pelagic net, which it probably entered in shallower water.

Eastern Atlantic off Morocco, 2083, 2075 meters, two specimens.

Distribution.-Mediterranean, abundant in 400-600 meters. Eastern Atlantic from the Azores and Portugal to ca. $11^{\circ} \mathrm{S} ., 13^{\circ} \mathrm{E}$. (off Angola, West Africa), three deep-abyssal records and many specimens in 410-1590 meters. Common between 500 and 800 meters, rare below 1000 meters. The species may be partially pelagic and thus may not actually have been caught in the deepabyssal zone.

Length. $-60-215+\mathrm{mm}$.

\section{Lionurus filicauda Günther}

Coryphaenoides filicauda Günther, 1878, Ann. Mag. Nat. Hist., (5), 2: 27 -type locality not stated, designated as Challenger Station 299, Pacific

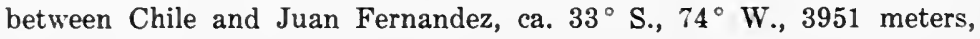
the locality of the specimen figured in Günther, 1887. 
Macrurus filicauda Günther, 1887, Rep. Sci. Res. Voy. Challenger, Zool., 22 : 141, pl. 34, fig. B.

Macrurus (Lionurus) filicauda Murray and Hjort, 1912, Depths of Ocean, p. 417 , fig. 306 .

Lionurus filicauda Regan, 1914, Brit. Antaretic (Terra Nova) Exp. 1910, Zool., 1, (1), p. 39; Gilbert and Hubbs, 1916, Proc. U. S. Nat. Mus., 51 : 146.

Deep-abyssal records and distribution.-South Atlantic, ca. $36^{\circ}$ S., $46^{\circ}$ W., 4846 meters, three specimens.

South Atlantic, ca. $35^{\circ}$ S., $50^{\circ}$ W., 3475 meters, one specimen.

Southeastern Pacific, ca. $33^{\circ}$ S., $74^{\circ}$ W., 3951 meters, one specimen.

South Indian Ocean, ca. $53^{\circ}$ S., $108^{\circ}$ E., 3566 meters, three specimens.

South Indian Ocean, ca. $50^{\circ}$ S., $123^{\circ}$ E., 3291 meters, two specimens.

South Indian Ocean, ca. $46^{\circ}$ S., $45^{\circ}$ E., 2515 meters, twelve specimens.

Known only from twenty-two specimens taken by the Challenger in southern seas. Occurring on the abyssal plain and probably confined to deep-abyssal waters.

Length. $-102-381 \mathrm{~mm}$.

\section{Lionurus liolepis Gilbert}

Macrurus (Lionurus) liolepis Gilbert, 1890, Proc. U. S. Nat. Mus., 13: 117

-Pacific off southern California (exact type locality not stated).

Lionurus liolepis Goode and Bean, 1895, Ocean. Ichth., p. 409; Jordan and Evermann, 1898, Bull. U. S. Nat. Mus., 47: 2593; Gilbert, 1915, Proc. U. S. Nat. Mus., 48: 376; Townsend and Nichols, 1925, Bull. Amer. Mus. Nat. Hist., $52: 17$.

Macrurus liolepis Gilbert, 1896, Rep. U. S. Comm. Fish, 1893 : 473; Garman, 1899, Mem. Mus. Comp. Zool., 24 : 199.

Deep-abyssal record.-Eastern Pacific off San Nicolas Island, southern California, 1982-2012 meters, (?) specimens.

Distribution. - Eastern Pacific from Cape San Lucas to Monterey Bay, abundant in 294-1655 meters; one deep-abyssal record. Center of distribution above 1000 meters, rarely taken below that depth. Accidental in deep-abyssal waters.

Length.-?-305 mm.

\section{Lionurus fragilis Garman}

Macrurus fragilis Garman, 1899, Mem. Mus. Comp. Zool., 24: 203, pl. 46, fig. 1-Pacific off Panama and Ecuador (type locality not designated). 
Lionurus fragilis Gilbert and Hubbs, 1916, Proc. U. S. Nat. Mus., 51 : 146.

Deep-abyssal records and distribution.-Eastern Pacific, ca. $2^{\circ} \mathrm{N}$., $83^{\circ}$ W., 3333 meters, (?) specimens.

Eastern Pacific, ca. $6^{\circ} \mathrm{N} ., 82^{\circ} \mathrm{W} ., 3057$ meters, (?) specimens. Length.-?-229 $\mathrm{mm}$.

\section{Nezumia convergens Garman}

Macrurus convergens Garman, 1899, Mem. Mus. Comp. Zool., 24: 210, pl. 48, fig. 1-Pacific off Panama (type locality not designated).

Lionurus (Nezumia) convergens Gilbert and Hubbs, 1916, Proc. U. S. Nat. Mus., 51 : 146.

Deep-abyssal record.-Eastern Pacific, ca. $7^{\circ}$ N., $79^{\circ}$ W., 1865 meters, (?) specimens.

Distribution.-Eastern Pacific, $6^{\circ}-7^{\circ} \mathrm{N}$, $79^{\circ}-81^{\circ} \mathrm{W}$., one deepabyssal record and an unstated number of specimens from two hauls in 1430 and 1271 meters.

Length.-Not stated.

\section{Nezumia bairdi Goode and Bean}

Macrurus Bairdii Goode and Bean, *1877, Amer. Jour. Sci., 14 : 471-Atlantic, Massachusetts Bay.

Nezumia bairdii Parr, 1946, Bull. Bingham Oceanogr. Coll., 10, (1), pp. 31, 37, figs. 9, 12 (full synonymy); Marshall, 1954, Aspects Deep Sea Biol., pp. 257-259, fig. X-2.

Macrourus bairdii Bigelow and Schroeder, 1953, Fish. Bull., Fish Wildlife Serv., 53, (74), p. 243, fig. 119; Scholander and Van Dam, 1953, Biol. Bull., 104: 76.

Deep-abyssal record.-Western Atlantic, ca. $41^{\circ} \mathrm{N}$., $66^{\circ} \mathrm{W} ., 2295$ meters, (?) specimens.

Distribution.-Eastern Atlantic, one specimen reported near the Azores in 1153 meters, a doubtful identification. Western Atlantic from the West Indies to the Grand Banks of Newfoundland, common and abundant. Vertical range very wide, one deep-abyssal record, otherwise 0-17 meters (three specimens) and 108-1748 meters. Center of distribution well above the deep-abyssal zone, in ca. $400-1000$ meters.

Length. $-150-400 \mathrm{~mm}$.

\section{Nezumia aequalis Günther}

Coryphaenoides aequalis Günther, 1878, Ann. Mag. Nat. Hist., (5), 2: 25 -Atlantic south of Portugal, ca. $36^{\circ}$ N., $8^{\circ}$ W., 1098 meters. 
?Macrurus serratus Lowe, ${ }^{*} 1843$, Proc. Zool. Soc. London, p. 91 ; Goode and Bean, 1895, Ocean. Ichth., pp. 392, 534.

?Coryphaenoides serratus Günther, 1862, Cat. Fishes Brit. Mus., 4: 396.

Macrurus aequalis Günther, 1887, Rep. Sci. Res. Voy. Challenger, Zool., 22: 134, pl. 32, fig. C; Goode and Bean, 1895, Ocean. Ichth., p. 392; Holt and Calderwood, 1895, Sci. Trans. Roy. Dublin Soc., 5, (9), p. 463, pl. 40, fig. 1; Koehler, 1896, Ann. Univ. Lyon, 26: 495; Collett, 1896, Rés. Camp. Sci. Monaco, 10: 75, pl. 2, fig. 9; 1905, Rep. Norwegian Fish. Mar. Invest., 2, (3), p. 55; Holt and Byrne, 1906, Rep. Fish. Ireland 1905, Sci. Invest., 2: 24; Zugmayer, 1911, Rés. Camp. Sci. Monaco, 35: 126; Murray and Hjort, 1912, Depths of Ocean, pp. 59, 397, pl. 8, fig. 2, text figs. 39, a, 270; Koefoed, 1927, Rep. Sci. Res. M. Sars No. Atl. Deep-sea Exp. 1910, 4, (1), p. 84, pl. 2, fig. 1, text figs. 19-21; Schnakenbeck, 1932, Faune Ichth. Atl. Nord, 12, fig.; Noronha and Sarmento, *1934, Peixes Madeira, p. 112; Lozano Rey, 1934, Bol. Soc. Esp. Hist. Nat., 34: 91; Brunelli and Bini, 1934, Boll. Pesca Pisc. Idrobiol., 10, (6), p. 13; Nobre, 1935, Fauna Marinha Portugal, 1: 507; Jensen, 1948, Skr. Univ. Zool. Mus. København, 9: 181.

Macrurus smiliophorus Vaillant, 1888, Exp. Sci. Trav. Talis., Poiss., p. 242, pl. 22, fig. 1; Goode and Bean, 1895, Ocean. Ichth., p. 392.

Coryphaenoides aequalis Vaillant, 1888, Exp. Sci. Trav. Talis., Poiss., p. 386. Macrurus serratus Roule, 1919, Rés. Camp. Sci. Monaco, 52: 79; Vaillant, in Roule, 1919, op. cit., p. 134; de Buen, 1935, Not. Res. Inst. Esp. Oceanogr., (2), 88: 72 .

Lionurus aequalis Farran, 1924, Proc. Roy. Irish Acad., B36: 113, pl. 7, fig. 5 , text fig. 6 .

Macruroplus serratus Fowler, 1936, Bull. Amer. Mus. Nat. Hist., 70: 464, fig. 225; Cadenat, 1954, Bull. Inst. Fr. Afrique Noire, 16: 568.

Nezumia aequalis Maul, 1951, Bol. Mus. Mun. Funchal, 5: 22, fig. 5.

Lionurus (Nezumia) aequalis Poll, 1953, Rés. Sci. Exp. Océanogr. Belge (19481949), 4, (2), (3), p. 240, fig. 97.

Deep-abyssal records.-Eastern Atlantic, ca. $43^{\circ}$ N., $9^{\circ}$ W., 2320 meters, three specimens.

Eastern Atlantic, ca. $31^{\circ} \mathrm{N}$., $10^{\circ} \mathrm{W} ., 2165$ meters, one specimen.

Distribution.-Eastern Atlantic from Faroe Bank (ca. $61^{\circ} \mathrm{N}$.) to the Azores and Angola, West Africa (ca. $11^{\circ} \mathrm{S}$.), two deep-abyssal records, others in 200-1805 meters; common, center of distribution in ca. 400-1000 meters. Mediterranean, two records, one in 500 meters. Northwestern Atlantic in Davis Strait, ca. $63^{\circ} \mathrm{N}$., one specimen, 860 meters. Accidental in deep-abyssal waters.

Length. $-110-360 \mathrm{~mm}$.

Nezumia sclerorhynchus Valenciennes. Table 19.

Lepidoleprus sclerorhynchus, Macrurus sclerorhynchus Valenciennes, in Webb and Berthelot, 1836-44, Ichth. Iles Canaries, p. 80, pl. 14, fig. 1-Canary Islands. 
Macrurus sclerorhynchus Günther, 1862, Cat. Fishes Brit. Mus., 4: 394; Vinciguerra, 1879, Ann. Mus. Civ. Stor. Nat. Genova, 14: 622, pl. 2; 1885, op. cit., 22: 459; Giglioli, *1884, Pelagos, pp. 197, 199, 208; Vaillant, 1888, Exp. Sci. Trav. Talis., Poiss., pp. 31, 237, pl. 22, fig. 2; Vaillant, in Roule, 1919, Rés. Camp. Sci. Monaco, 52: 134; Steindachner, 1891, Sitz.-Ber. K. Akad. Wiss., 100: 442 ; Carus, 1893, Prodr. Faunae Medit., 2: 583; Goode and Bean, 1895, Ocean. Ichth., p. 391; Collett, 1896, Rés. Camp. Sci. Monaco, 10: 79; Murray and Hjort, 1912, Depths of Ocean, p. 397; Roule, 1919, Rés. Camp. Sci. Monaco, 52: 79; Koefoed, 1927, Rep. Sci. Res. M. Sars No. Atl. Deep-sea Exp. 1910, 4, (1), p. 84, figs. 17, 18; Sanzo, 1933, Monogr. Fauna Flora Golfo Napoli, 38: 261, pl. 16, figs. 9-11; Tortonese and Trotti, 1949, Atti Accad. Ligure Sci. Lett., 6, (1), p. 59 (further Mediterranean references).

Lionurus (Nezumia) sclerorhynchus Gilbert and Hubbs, 1916, Proc. U. S. Nat. Mus., 51: 146; Poll, 1953, Rés. Sci. Exp. Océanogr. Belge (19481949), 4, (2), (3), p. 243, fig. 98.

Macruroplus sclerorhynchus Fowler, 1936, Bull. Amer. Mus. Nat. Hist., 70: 462; Trotti, 1947, Ann. Mus. Civ. Stor. Nat. Genova, 68: 41.

Macrurus (Macrurus) sclerorhynchus Nybelin, 1948, Göteborgs K. Vetensk. Vitt.-Samh. Handl., (B), 5, (16), p. 65.

Deep-abyssal records.-Eastern Atlantic off Cape Verde, 3655 meters, three specimens.

Eastern Atlantic off Cape Verde, 3200 meters, three specimens.

Eastern Atlantic off Morocco, 2600 meters, one specimen.

Eastern Atlantic off Morocco, the Banc d'Arguin (North Africa) and off the Azores, 2210-2330 meters, twelve specimens from five hauls.

Eastern Atlantic, ca. $35^{\circ} \mathrm{N} ., 8^{\circ} \mathrm{W}$. and off the Canary Islands, 2055, 2015 meters, two specimens.

Eastern Atlantic off the Azores, ca. $39^{\circ}$ N., $29^{\circ}-30^{\circ}$ W., 1900 , 1846 meters, two specimens.

Distribution.-Eastern Atlantic from the Azores to ca. $8^{\circ} \mathrm{S}$.; numerous specimens in 540-1550 meters and twenty-three from deep-abyssal waters. Mediterranean, 500-600 meters, and one individual found floating at the surface, nearly dead. Relatively common, center of distribution in the Atlantic in ca. 800-1600 meters, with perhaps another, smaller area of abundance in 2200 2300 meters. ?Indian Ocean (see below).

Length. $-152-325 \mathrm{~mm}$.

Remarks.-Brauer (1906, p. 265) identified with this species a specimen taken in the Gulf of Aden at a depth of 1840 meters. Koefoed (1927, p. 87) referred Brauer's specimen to Chalinura guentheri Vaillant, but Nybelin $(1948$, p. 65) doubted the wisdom of 
this step. The actual range of the species therefore remains in some doubt.

\section{Nezumia parvipes Smith and Radcliffe}

Macrourus parvipes Smith and Radcliffe, in Radcliffe, 1912, Proc. U. S. Nat. Mus., 43: 124, pl. 28, fig. 1-Pacific near Gomono Island, Dutch East Indies, 2308 meters.

Lionurus (Nezumia) parvipes Gilbert and Hubbs, 1916, op. cit., 51: 146.

Lionurus parvipes Gilbert and Hubbs, 1920, Bull. U. S. Nat. Mus., 100, (1), p. 562.

Deep-abyssal records and distribution.-Near Gomono Island, Dutch East Indies, 2308 meters, two specimens.

Macassar Strait, 2160 meters, one specimen.

Gulf of Tomini, Celebes, 1997, 1992 meters, three specimens.

Western Pacific, Dutch East Indies. Exclusively deep-abyssal so far as known.

Length.-?-228 $\mathrm{mm}$.

\section{Cetonurus globiceps Vaillant}

Macrurus globiceps Vaillant, in Filhol, ${ }^{*} 1884$, Nature, Paris, 558: 185, fig. 2 -Atlantic off France, North Africa and the Azores (type locality not designated; see footnote, p. 110).

Hymenocephalus crassiceps Vaillant, 1888, Exp. Sci. Trav. Talis., Poiss., p. 214, pl. 20, fig. 1 .

Hymenocephalus globiceps Vaillant, op. cit., p. 386; Vaillant, in Roule, 1919, Rés. Camp. Sci. Monaco, 52: 134.

Cetonurus globiceps Goode and Bean, 1895, Ocean. Ichth., p. 411, fig. 344; Gilbert and Hubbs, 1916, Proc. U. S. Nat. Mus., 51 : 208; Fowler, 1936, Bull. Amer. Mus. Nat. Hist., 70: 467, fig. 227; Parr, 1946, Bull. Bingham Oceanogr. Coll., 10, (1), pp. 31, 43, figs. 9, 14.

?Macrurus (Cetonurus) globiceps Murray and Hjort, 1912, Depths of Ocean, p. 398 , fig. 271.

?Macrurus (Cetonurus) sp. Koefoed, 1927, Rep. Sci. Res. M. Sars. No. Atl. Deep-sea Exp. 1910, 4, (1), p. 96, pl. 5, fig. 9, text figs. 30-33.

Deep-abyssal records.-Eastern Atlantic, ca. $36^{\circ}$ N., $22^{\circ}$ W., 4621 meters, two specimens.

Eastern Atlantic off the Azores, 2995 meters, one specimen.

Distribution.-Eastern Atlantic from the Gulf of Gascony to the Canary Islands, two deep-abyssal records and eighteen specimens in 1139-1600 meters. Western Atlantic, ca. $16^{\circ} \mathrm{N}$., $63^{\circ} \mathrm{W}$., one specimen, 1257 meters.

Length.-?-350 $\mathrm{mm}$. 


\section{Echinomacrurus mollis Roule}

Echinomacrurus mollis Roule, 1916, Bull. Inst. Océanogr. Monaco, 320: 22 -Atlantic, ca. $31^{\circ} \mathrm{N} ., 24^{\circ} \mathrm{W} ., 5413$ meters.

Cetonurus microps Vaillant, in Richard, 1905, Bull. Inst. Océanogr. Monaco, 41 : 3; Fowler, 1936, Bull. Amer. Mus. Nat. Hist., $70: 468$ (nomen nudum).

Echinomacrurus mollis Roule, 1919, Rés. Camp. Sci. Monaco, 52: 84, pl. 4, fig. 2; Belloc, 1949, Bull. Inst. Océanogr. Monaco, 958: 16; ?Nybelin, 1951, Rep. Swedish Deep-sea Exp. 1947-1948, Zool., 2, (1), p. 25.

Deep-abyssal records and distribution.-Eastern Atlantic, 5413 meters, the type.

Eastern Atlantic, ca. $43^{\circ}$ N., $18^{\circ}$ W., 5000-5025 meters, two specimens, a doubtful identification.

Length.--?-375 $\mathrm{mm}$.

\section{Family STEPHANOBERYCIDAE}

The three species comprising this family are included in the benthic fauna for the present, although Brauer considered them to be bathypelagic. They have been caught only in trawls or dredges fishing on bottom. Two have been taken on the abyssal plain.

\section{Stephanoberyx monae Gill}

Stephanoberyx monae Gill, 1883, Proc. U. S. Nat. Mus., 6: 258-Atlantic off United States coast, ca. $41^{\circ} \mathrm{N} ., 66^{\circ} \mathrm{W} ., 2295$ meters.

Stephanoberyx monae Goode and Bean, 1895, Ocean. Ichth., p. 186, fig. 205, text fig.; Jordan and Evermann, 1896, Bull. U. S. Nat. Mus., 47: 836; 1900 , op. cit., fig. 353; Boulenger, 1904, Cambridge Nat. Hist., 7: 620; Myers, 1936, Copeia, p. 118.

Deep-abyssal record.-Western Atlantic, 2295 meters, the type.

Distribution.-Western Atlantic, one deep-abyssal record in ca. $41^{\circ} \mathrm{N}$.; around the West Indies $\left(15^{\circ}-16^{\circ} \mathrm{N} ., 61^{\circ}-63^{\circ} \mathrm{W}\right.$.) and in the Gulf of Mexico, an unstated number of specimens from seven hauls, 979-1766 meters.

Length.--?-51 mm.

\section{Acanthochaenus luetkeni Gill}

Acanthochaenus lutkeni Gill, 1884, Amer. Nat., 18:443-Atlantic, ca. $37^{\circ} \mathrm{N}$, $69^{\circ}$ W., 5393 meters.

Stephanoberyx gillii Goode and Bean, 1895, Ocean. Ichth., p. 187, fig. 206; Jordan and Evermann, 1896, Bull. U. S. Nat. Mus., 47: 836; Koefoed, 1927, Rep. Sci. Res. M. Sars No. Atl. Deep-sea Exp. 1910, 4, (1), p. 65.

Acanthochaenus lutkenii Myers, 1936, Copeia, p. 18. 
Deep-abyssal records and distribution.-Western Atlantic, 5393 meters, the type.

Western Atlantic, $38^{\circ}-39^{\circ}$ N., $69^{\circ}-72^{\circ}$ W., 2250,2211 meters, three or more specimens from two hauls.

Eastern Atlantic, ca. $34^{\circ}$ N., $33^{\circ}$ W., 2615-2865 meters, one specimen.

Eastern Atlantic, ca. $27^{\circ} \mathrm{N}$., $14^{\circ} \mathrm{W} ., 2603$ meters, two specimens. Length. $-120-152 \mathrm{~mm}$.

\section{Malacosarcus macrostoma Günther}

Scopelus macrostoma Günther, 1878, Ann. Mag. Nat. Hist., (5), 2: $186-$ Pacific, ca. $0^{\circ} 33^{\prime}$ S., $151^{\circ}$ W., 4435 meters.

Malacosarcus macrostoma Günther, 1887, Rep. Sci. Res. Voy. Challenger, Zool., 22 : 30; Goode and Bean, 1895, Ocean. Ichth., p. 182.

Deep-abyssal records and distribution.-Mid-Pacific, ca. $0^{\circ} 33^{\prime}$ S., $151^{\circ} \mathrm{W} ., 4435$ meters, one specimen.

Mid-Pacific, ca. $13^{\circ}$ S., $149^{\circ}$ W., 4298 meters, one specimen.

Length. $-89 \mathrm{~mm}$.

\section{Family MELAMPHAIDAE}

Melamphaids are presumably all bathypelagic fishes, cosmopolitan in temperate and tropical latitudes, and seldom caught above a depth of 1000 meters. There are about twenty-seven supposedly valid species of the genus Melamphaes but the synonymy of many is somewhat confused, and only $M$. nigrescens Brauer can be placed below 2000 meters with any degree of certainty. Even that species may be synonymous with $M$. robustus Günther, an Atlantic species found by Beebe (1937, p. 206) and Parr (1933, p. 19) to be abundant above 2000 meters; and in this connection it is interesting to note that $M$. robustus was once taken off South Africa in a closing-net at a depth of 2850 meters (Norman, 1930, p. 345).

Considering the tendency toward a deep bathypelagic habitat exhibited by the family as a whole, it will not be surprising if some species prove to inhabit deep-abyssal waters.

Melamphaes nigrescens Brauer. Table 20.

Melamphaes nigrescens Brauer, 1906, Wiss. Ergebn. Deutschen Tiefsee Exp. Valdivia, 15, (1), p. 283, pl. 13, fig. 4-Atlantic, Gulf of Guinea and off South Africa, and Indian Ocean, various localities (type locality not designated). 
Scopelus crassiceps Günther, 1878, Ann. Mag. Nat. Hist., (5), 2: 185 (part).

Melamphaes crassiceps Günther, 1887, Rep. Sci. Res. Voy. Challenger, Zool., 22: 28 (part); ?Koumans, 1953, Temminckia, 9: 216.

?Melamphaes nigrescens Zugmayer, 1911, Rés. Camp. Sci. Monaco, 35: 99.

?Melamphaes nycterinus Gilbert, 1915, Proc. U. S. Nat. Mus., 48: 326, pl. 16, fig. 6 .

Melamphaes nigrescens Beebe, 1926, Arcturus Adv., p. 409; 1929, Zoologica, 12: 18; Norman, 1929, Ann. Mag. Nat. Hist., (10), 4: 160, 161; 1939, Sci. Rep. John Murray Exp. 1933-34, 7, (1), p. 56; Parr, 1931, Bull. Bingham Oceanogr. Coll., 2, (4), p. 40; 1933, op. cit., 3, (6), p. 14; Fowler, 1936, Bull. Amer. Mus. Nat. Hist., 70: 1265; Koefoed, 1953, Rep. Sci. Res. M. Sars No. Atl. Deep-sea Exp. 1910, 4, (2), (3), p. 19.

Melamphaes robustus Norman, 1939, Sci. Rep. John Murray Exp. 1933-34, $7,(1)$, p. 56.

Distribution.-Eastern Atlantic from ca. $48^{\circ} \mathrm{N} ., 13^{\circ} \mathrm{W}$. to ca. $31^{\circ}$ S., $9^{\circ}$ E. (off South Africa). Mid-Atlantic, ca. $48^{\circ}-31^{\circ} \mathrm{N}$. Western Atlantic off New York. Indian Ocean from ca. $34^{\circ} \mathrm{S}$., $80^{\circ}$ E. and ca. $26^{\circ} \mathrm{S} ., 93^{\circ} \mathrm{E}$. to the Bay of Bengal, the Arabian Sea, and ca. $4^{\circ}$ S., $48^{\circ}$ E. Western Pacific north of New Guinea (ca. $0^{\circ} 42^{\prime}$ S., $147^{\circ}$ E.) and in (?) the Flores Sea $\left(7^{\circ}-8^{\circ}\right.$ S., $118^{\circ}-129^{\circ}$ E.). Eastern Pacific, ca. $6^{\circ} \mathrm{N}$., $85^{\circ} \mathrm{W}$. Known from only forty-nine specimens taken in twenty-seven hauls made between 1098 and 4000 meters. Chief area of occurrence in about 2000 meters.

Length. $-30-80 \mathrm{~mm}$.

Remarks.-Norman (1929, p. 160) believed Gilbert's M. nycterinus, known from a single specimen taken off Catalina Island (eastern Pacific) in 3865-4132 meters, to be synonymous with nigrescens; and he suspected that Zugmayer's three specimens identified as nigrescens (eastern Atlantic, 5100 meters) might actually be robustus.

\section{Family ACROPOMATIDAE(?)}

The following species belongs to a group of fishes (Apogonidae, Scombropidae, Acropomatidae) common among corals and weeds, with a world-wide distribution in tropical and temperate seas and with a few deep-water species. Some of the latter are known to be bathypelagic, others probably benthic, but only Brephostoma carpenteri has been taken in deep-abyssal waters.

\section{Brephostoma carpenteri Alcock}

Brephostoma carpenteri Alcock, 1889, Ann. Mag. Nat. Hist., (6), 4: 383Indian Ocean, ca. $6^{\circ} \mathrm{N} ., 90^{\circ} \mathrm{E}$. 
Brephostoma carpenteri Alcock, 1890, Ann. Mag. Nat. Hist., (6), 6: 201, pl. 9, fig. 4; 1899, Descr. Cat. Indian Deep-sea Fishes, p. 26; Goode and Bean, 1895, Ocean. Ichth., p. 234; Alcock and Anderson, 1898, Ill. Zool. Investigator, Fishes, pl. 18, fig. 2.

Deep-abyssal record and distribution.-Indian Ocean, Bay of Bengal, 2505-2780 meters, one specimen.

Length. $-102 \mathrm{~mm}$.

Remarks. - The small amount of evidence at hand points to a habitat on bottom rather than in mid-water. According to Alcock $(1889$, p. 383), the fact that the specimen was embedded in the head of one of the swabs attached to the trawl suggests that the swab settled over the fish as it lay on the bottom.

\section{Family PARAPERCIDAE}

Like Brephostoma carpenteri (above), Macrias amissus is the only member of its family found at any great depth. Related species are all bottom-living forms from tropical and temperate seas, a few of them archibenthic but the majority inhabitants of the shore.

Macrias amissus Gill and Townsend

Macrias amissus Gill and Townsend, 1901, Science, 14: 937-Pacific off southern Chile, ca. $45^{\circ} \mathrm{S} ., 75^{\circ} \mathrm{W}$.

Macrias amissus Townsend, 1936, Bull. N. Y. Zool. Soc., 39: 29, fig.

Deep-abyssal record and distribution.-Southeastern Pacific, 1920 meters, one specimen.

Length.-Ca. $1524 \mathrm{~mm}$. (5 ft.).

Remarks.-This specimen is startling proof of how little is known of the animals inhabiting the deeper parts of the ocean. Unfortunately it was thrown overboard without the knowledge of the Albatross biologists and there remains only a photograph to represent the type.

\section{Family CHIASMODONTIDAE}

Although five species have been recorded exclusively in deepabyssal waters, they are known from only one specimen each (in one case, two specimens) and probably are bathypelagic in habitat. The only specimens taken in closing nets were the type and only known example of Kali macrodon Norman (off South Africa, 2500 2000 meters) and one specimen of Dysalotus alcocki MacGilchrist (Atlantic, ca. $38^{\circ} \mathrm{S} ., 28^{\circ} \mathrm{W} ., 1800-2000$ meters). This last form is 
known from only three other specimens, the type from the Bay of Bengal in 1289 meters and two western Atlantic examples caught in 1000 and 1609 meters.

Chiasmodonts have not been taken as abundantly as melamphaids and it is not possible to determine whether the family as a whole shows a similar tendency toward a life below 1000 meters. The family distribution is cosmopolitan in tropical and temperate seas.

\section{Family BATHYDRACONIDAE}

The two species listed below are the only nototheniiform fishes reported from deep-abyssal waters. Few members of the group, which is confined to the southern hemisphere, are found even below a depth of 1000 meters.

\section{Bathydraco antarcticus Günther}

Bathydraco antarcticus Günther, 1878, Ann. Mag. Nat. Hist., (5), 2: 18 Antarctic, ca. $60^{\circ} \mathrm{S} ., 80^{\circ} \mathrm{E}$.

Bathydraco antarcticus Günther, 1887, Rep. Sci. Res. Voy. Challenger, Zool., 22: 48, pl. 8, fig. A; Regan, 1913, Trans. Roy. Soc. Edinburgh, 49: 282; Norman, 1938, Disc. Rep., 18: 55.

Deep-abyssal record and distribution.-Antarctic south of Heard Island, 2304 meters, one specimen.

Length.-260 $\mathrm{mm}$.

\section{Bathydraco scotiae Dollo}

Bathydraco scotiae Dollo, 1906, Proc. Roy. Soc. Edinburgh, 26: 65-Antarctic, ca. $71^{\circ} \mathrm{S} ., 16^{\circ} \mathrm{W} ., 2579$ meters.

Bathydraco scotiae Regan, 1913, Trans. Roy. Soc. Edinburgh, 49: 240, 282, pl. 9, fig. 4; Norman, 1937, Rep. B.A.N.Z. Antarctic Res. Exp. 1929-1931, (B), 1, (2), p. 74; 1938, Disc. Rep., 18: 56.

Deep-abyssal records and distribution.-Antarctic off Coats Land, 2579 meters, two specimens.

Antarctic, ca. $64^{\circ} \mathrm{S}$, $116^{\circ}$ E., 2260 meters, one specimen.

Length. $-133-145 \mathrm{~mm}$.

\section{Family ZOARCIDAE}

Zoarcids form a large part of the benthic fauna of Arctic and Antarctic seas, in both littoral and deep-sea zones. To a lesser degree they are found also in some temperate latitudes, and even 
extend, in deep water, to the tropical eastern Pacific, where there are some of the deepest records of these fishes. All but four of the sixteen species (seven genera) recorded from deep-abyssal waters are represented by only one or two specimens each. Of the three better-known forms only Lycodes frigidus has a wide geographical distribution, the other two being rather restricted in occurrence. Some zoarcids are known to move into deeper water with age. Two species have been recorded from the abyssal plain.

\section{Lycodes frigidus Collett. Table 21.}

Lycodes frigidus Collett, ${ }^{*} 1878$, Vidensk. Selsk. Forh., 1878, (14), p. 45-Arctic, west of Hammerfest, Norway, 2030 meters. $^{1}$

Lycodes vahlii Collett, op. cit., p. 11 (part).

Lycodes frigidus Collett, 1880, Norske Nordhavs Exp. 1876-78, Zool., Fiske, p. 96, pl. 3, figs. 23, 24; 1905, Rep. Norwegian Fish Mar. Invest., 2, (3), p. 137; Günther, 1887, Rep. Sci. Res. Voy. Challenger, Zool., 22: 79; Lilljeborg, *1891, Sveriges Norges Fauna, Fisk., 2: 19; Smitt, 1895, Skand. Fiskar, p. 610, fig. 146; Lütken, 1898, Danish Ingolf Exp., 2, (1), p. 20 (part); Lönnberg, 1899, Bih. K. Svensk. Vetensk. Akad. Handl., 24, (4), (9), p. 24; 1900, Rev. Intern. Pêche Pisc., 2, (4), p. 13; Ehrenbaum, 1902, Fauna Arct., 2: 120; Jensen, 1902, Vidensk. Medd. Dansk naturh. Foren., 1901: 213; 1904, Danish Ingolf Exp., 2, (4), pp. 22, 98, pl. 5, fig. 1; Vaillant, in Roule, 1919, Rés. Camp. Sci. Monaco, 52: 133; Johnsen, 1921, Bergens Mus. Aarb., 1918-19, (6), p. 35; Barnard, 1927, Ann. So. Afr. Mus., 21 : 872; Smith, 1949, Sea Fishes So. Afr., p. 365, fig. 1026; Andriashev, 1954, Fauna'S.S.S.R., 53: 302, fig. 172.

Lycodes reticulatus Günther, 1887, Rep. Sci. Res. Voy. Challenger, Zool., 22: 77 , pl. 13.

Lycodes reticulatus f. frigida Smitt, 1901, Bih. K. Svensk. Akad. Handl., 27, (4), (4), p. 29 (part).

?Lycodes sp. Essipov, 1939, Zool. Zhur., 18, (5), pp. 877, 886.

Deep-abyssal records.-Arctic west of Spitzbergen, 2750, 2438 meters, four specimens from two hauls.

Arctic, $67^{\circ}-69^{\circ}$ N., $6^{\circ}-7^{\circ}$ W., 2394,2315 meters, seven specimens from two hauls.

Arctic from west of Hammerfest to near Jan Mayen, 20002195 meters, ten specimens from four hauls.

Arctic, $67^{\circ}-78^{\circ}$ N., $5^{\circ}$ E. $-10^{\circ}$ W., 1835-1939 meters, twentyeight specimens from four hauls.

${ }^{1}$ I have not seen the original description. Collett (1880, p. 96) did not specify a type locality but stated that the only adult specimen taken was from Voringen Station 295, the locality of which, according to Sanderson Smith (1888, p. 988) was $71^{\circ} 59^{\prime} \mathrm{N}$., $11^{\circ} 40^{\prime} \mathrm{E}$. 
(?)Arctic west of Spitzbergen, 2880, 3000 meters, two specimens. ${ }^{1}$

Distribution.-Arctic from ca. $80^{\circ}$ N., $3^{\circ}$ E. and $78^{\circ}$ N., $5^{\circ}$ E. to near Iceland and the Faroes (ca. $62^{\circ} \mathrm{N}$.), twelve (possibly fourteen) deep-abyssal records; others in 836-1780 meters. Not rare. Center of distribution apparently between 1600 and 2400 meters. Off Cape Point, South Africa, (?) specimens, 631-1645 meters.

\section{Length. $-46-558 \mathrm{~mm}$.}

Remarks.--Jensen $(1904$, p. 25) found that the specimen reported by Collett (1880, p. 96) in 640 meters is not frigidus and stated that Collett's record from 475 meters may also be an error. Jensen's studies placed the upper limit of the species, in the Arctic, at about 1000 meters.

\section{Lycodes atlanticus Jensen. Table 22.}

Lycodes atlanticus Jensen, 1902, Vidensk. Medd. Dansk naturh. Foren., 1901: 207-Atlantic off United States coast (type locality not stated).

Lycodes frigidus Goode and Bean, 1895, Ocean. Ichth., p. 305, fig. 274 ; Jordan and Evermann, 1898, Bull. U. S. Nat. Mus., 47: 2465; 1900, op. cit., fig. 856.

Lycodes atlanticus Jensen, 1904, Danish Ingolf Exp., 2, (4), p. 25; Bigelow and Welsh, 1925, Bull. U. S. Bur. Fish., 40, (1), p. 383; Bigelow and Schroeder, 1953, Fish. Bull., Fish Wildlife Serv., 53: 509.

Lycodes terrae-novae Vladykov and Tremblay, 1936, Stat. Biol. St. Laurent, Faune Flore Laurent., 1 : 11 (part).

Deep-abyssal records.-Western Atlantic off coast of United States, $37^{\circ}-39^{\circ}$ N., 2603, 2155, 1869-2021 meters, an unstated number of specimens from eight hauls.

Distribution.-Western Atlantic off coast of United States, $35^{\circ}-$ $41^{\circ} \mathrm{N}$., eight deep-abyssal records and an unstated number of specimens from thirteen hauls in 944-1761 meters. Center of distribution in about 1500-2200 meters with stragglers down to 2600 meters and up to 900 meters.

Length.--?-338 mm.

\section{Lycodes platyrhinus Jensen}

Lycodes platyrhinus Jensen, 1902, Vidensk. Medd. Dansk naturh. Foren., 1901: 208-Arctic, ca. $67^{\circ} \mathrm{N} ., 10^{\circ} \mathrm{W}$.

Lycodes frigidus Lütken, 1898, Danish Ingolf Exp., 2, (1), p. 21 (part).

Lycodes platyrhinus Jensen, 1904, op. cit., 2, (4), p. 51, pl. 6, fig. 2, text figs. 3-5.

${ }^{1}$ Lycodes sp. Essipov (1939, pp. 877, 886), perhaps L. frigidus, fide Andriashev (1954, p. 302). 
Lycenchelys(?) platyrhinus Andriashev, 1954, Fauna S.S.S.R., 53: 308, figs. $173,174$.

Deep-abyssal record and distribution.-Arctic between Jan Mayen and Iceland, 1847 meters, one specimen.

Length. $-148.5 \mathrm{~mm}$.

\section{Lycodes cicatrifer Garman}

Lycodes cicatrifer Garman, 1899, Mem. Mus. Comp. Zool., 24: 136, pl. 31, fig. 1-Pacific off Panama, ca. $6^{\circ}$ N., $82^{\circ} \mathrm{W}$.

Deep-abyssal record and distribution.-Eastern Pacific, 3057 meters, (?) specimens

Length.-?-235 $\mathrm{mm}$.

\section{Lycenchelys jordani Evermann and Goldsborough}

Lycodes jordani Evermann and Goldsborough, 1907, Bull. U. S. Bur. Fish., 26: 343, fig. 120-Pacific off coast of United States, ca. $43^{\circ} \mathrm{N} ., 125^{\circ} \mathrm{W}$., 1945 meters.

Lycenchelys jordani Taranetz, 1937, Bull. Pac. Sci. Inst. Fish. Oceanogr., $11: 161$.

Lycodes jordani Böhlke, 1953, Stanf. Ichth. Bull., 5: 101.

Deep-abyssal record.-Northeastern Pacific, 1945 meters, one specimen.

Distribution.-Northeastern Pacific, one deep-abyssal record and one specimen off Sitka Sound, ca. $57^{\circ}$ N., in 1687 meters.

Length.-Ca. 215-336 mm.

\section{Lycenchelys antarcticus Regan}

Lycenchelys antarcticus Regan, 1913, Trans. Roy. Soc. Edinburgh, 49: 243, pl. 9, fig. 3-Antarctic, ca. $62^{\circ}$ S., $41^{\circ} \mathrm{W}$.

Lycenchelys antarcticus Norman, 1938, Disc. Rep., 18: 81, fig. 52.

Deep-abyssal record and distribution.-Antarctic near the South Orkney Islands, 3246 meters, one specimen.

Length. $-128 \mathrm{~mm}$.

\section{Lycenchelys albus Vaillant}

Lycodes albus Vaillant, 1888, Exp. Sci. Trav. Talis., Poiss., p. 391, pl. 26, fig. 1-between France and the Azores, Talisman Station 133 (see footnote, p. 110).

Lycenchelys albus Goode and Bean, 1895, Ocean. Ichth., p. 526; Fowler, 1936, Bull. Amer. Mus. Nat. Hist., 70: 1055, fig. 436. 
Deep-abyssal record and distribution.--Eastern Atlantic, 3975 meters, two specimens.

Length.-?-199 $\mathrm{mm}$.

\section{Lycodonus ophidium Jensen}

Lycenchelys ophidium Jensen, 1902, Vidensk. Medd. Dansk naturh. Foren., 1901 : 212 -Atlantic south of Iceland, ca. $61^{\circ} \mathrm{N} ., 19^{\circ} \mathrm{W}$.

Lycodes muraena Lütken, 1898, Danish Ingolf Exp., 2, (1), p. 20 (part).

Lycodonus ophidium Jensen, 1904, op. cit., 2, (4), p. 97.

Deep-abyssal record and distribution.-North Atlantic, 1992 meters, one specimen.

Length. $-118 \mathrm{~mm}$.

Lycodonus mirabilis Goode and Bean. Table 23.

Lycodonus mirabilis Goode and Bean, 1883, Bull. Mus. Comp. Zool., 10: 208-Atlantic off United States coast, ca, $38^{\circ}$ N., $73^{\circ}$ W., 1353 meters.

Lycodonus mirabilis Günther, 1887, Rep. Sci. Res. Voy. Challenger, Zool., 22: 81; Goode and Bean, 1895, Ocean. Ichth., p. 312, fig. 280; Jordan and Evermann, 1898, Bull. U. S. Nat. Mus., 47: 2474; 1900, op. cit., fig. 862; Koefoed, 1927, Rep. Sci. Res. M. Sars No. Atl. Deep-sea Exp. 1910, 4, (1), p. 137, pl. 6, fig. 6; Jensen, 1952, Medd. Grønland, 142, (7), p. 27, pl. 1, fig. 2, $a$, pl. 2, figs. 2, $b-d$.

Deep-abyssal records.-Western Atlantic off coast of United States, 2394, 2295 meters, an unstated number of specimens from two hauls.

Western Atlantic off coast of United States, 1869-1977 meters, an unstated number of specimens from five hauls.

Distribution.-Western Atlantic south of Newfoundland (ca. $42^{\circ}$ N., $51^{\circ}$ W.) and off the United States coast $\left(35^{\circ}-41^{\circ}\right.$ N.), seven deep-abyssal records and an unknown number of specimens from ten hauls in 1100-1813 meters. Baffin Bay, two specimens in 850 meters. Apparently an inhabitant of the upper deep-abyssal zone, center of distribution perhaps in 1600-2000 meters. The species has been caught above 1550 meters only four times and in three of these hauls $(850,1100$ and 1353 meters) the specimens were young. In the fourth instance the length is not known.

Length. $-70-290 \mathrm{~mm}$.

\section{Lycodonus flagellicauda Jensen}

Lycenchelys flagellicauda Jensen, 1902, Vidensk. Medd. Dansk naturh. Foren., 1901 : 210 -Arctic west of Spitzbergen, ca. $79^{\circ}$ N., $5^{\circ}$ E., 839 meters. 
Lycodes muraena Collett, ${ }^{*} 1878$, Vidensk. Selsk. Forh., 1878, (14), p. 74 (part); 1880, Norske Nordhavs Exp., Zool., Fiske, p. 116 (part), pl. 4, figs. 29, 31; Günther, 1887, Rep. Sci. Res. Voy. Challenger, Zool., 22: 79, pl. 12, fig. A; Lilljeborg, *1891, Sveriges Norges Fauna, Fisk., 2: 25 (part); Smitt, 1895, Skand. Fiskar, 2: 616 (part), fig. 152; Lütken, 1898, Danish Ingolf Exp., 2, (1), p. 20 (part).

Lycodonus flagellicauda Jensen, 1904, op. cit., 2, (4), p. 94, figs. 29-33; 1938, Faune Ichth. Atl. Nord, 18, figs. 1-5; Collett, 1905, Rep. Norwegian Fish. Mar. Invest., 2, (3), p. 140; Koefoed, 1927, Rep. Sci. Res. M. Sars No. Atl. Deep-sea Exp. 1910, 4, (1), p. 136.

Deep-abyssal record.-Arctic, ca. $69^{\circ}$ N., $8^{\circ}$ W., 1835 meters, one specimen.

Distribution.-Arctic only, from near Spitzbergen (ca. $79^{\circ} \mathrm{N}$.) to north of Iceland and in the Faroe Channel (ca. $60^{\circ} \mathrm{N}$.), one deepabyssal record, five examples in 1750 meters and numerous specimens in 839-1394 meters. Probably not a deep-abyssal species.

Length. $-70-217 \mathrm{~mm}$.

\section{Maynea bulbiceps Garman}

Maynea bulbiceps Garman, 1899, Mem. Mus. Comp. Zool., 24: 140, pl. E,

fig. 1-Pacific off Panama, ca. $6^{\circ} \mathrm{N} ., 86^{\circ} \mathrm{W}$.

Maynea bulbiceps Gilbert, 1915, Proc. U. S. Nat. Mus., 48: 364.

Deep-abyssal record and distribution.-Eastern Pacific, 2690 meters, (?) specimens.

Length. $-502 \mathrm{~mm}$.

\section{Maynea conorhynchus Garman}

Gymnelis conorhynchus Garman, 1899, Mem. Mus. Comp. Zool., 24: 131, pl. 31, fig. 2-Pacific off Panama, ca. $6^{\circ} \mathrm{N}$., $80^{\circ} \mathrm{W}$.

Maynea conorhynchus Gilbert, 1915, Proc. U. S. Nat. Mus., 48: 364.

Deep-abyssal record and distribution.-Eastern Pacific, 3279 meters, (?) specimens.

Length. $-229 \mathrm{~mm}$.

\section{Bothrocaropsis elongata Garman}

Bothrocaropsis elongata Garman, 1899, Mem. Mus. Comp. Zool., 24: 129, pl. 33, fig. 2-Pacific off Panama, ca. $7^{\circ} \mathrm{N}$., $79^{\circ}-80^{\circ} \mathrm{W}$.

Deep-abyssal record.-Eastern Pacific off Panama, 1865 meters, an unstated number of specimens.

Distribution.-Eastern Pacific off Panama, one deep-abyssal record and an unstated number of specimens in 1271 meters.

Length.—-305 $\mathrm{mm}$. 


\section{Melanostigma pammelas Gilbert}

Melanostigma pammelas Gilbert, 1896, Rep. U. S. Comm. Fish, 1893: 472, pl. 35-Pacific off California, ca. $36^{\circ} \mathrm{N}$., $121^{\circ}$ W., 698 meters.

Melanostigma pammelas Jordan and Evermann, 1898, Bull. U. S. Nat. Mus., 47: 2479, 2869; Evermann and Goldsborough, 1907, Bull. U. S. Bur. Fish., 26: 345, fig. 124; Gilbert, 1915, Proc. U. S. Nat. Mus., 48: 368; Townsend and Nichols, 1925, Bull. Amer. Mus. Nat. Hist., 52: 15; Bolin and Myers, 1950, Stanf. Ichth. Bull., 3: 203-208; Böhlke, 1953, op. cit., 5: 101.

Deep-abyssal record.-Eastern Pacific off San Nicolas Island, ca. $33^{\circ}$ N., 1982-2012 meters, an unstated number of specimens from one haul.

Distribution.-Eastern Pacific from Monterey Bay to off San Diego, one deep-abyssal record and twenty-two specimens taken between 97-216 meters and 1398-1629 meters. An archibenthic form. Rare below 1000 meters and probably accidental in deepabyssal waters.

\section{Length.-56-304 mm.}

Remarks.-Gilbert (1915, p. 368) stated that the species may be pelagic. Bolin and Myers (1950, pp. 203-208) reported two captures in nets that brought up several bathypelagic fishes, thus adding evidence to support Gilbert's suggestion and raising considerable doubt of the validity of the one deep-abyssal record. However, twenty of the twenty-two specimens were taken in bottom-fishing appliances, with as many as three examples per haul, and the species is therefore included here in the list of benthic forms.

$M$. pammelas and $M$. atlanticum may be synonymous.

\section{Melanostigma atlanticum Koefoed}

Melanostigma atlanticum Koefoed, 1952, Rep. Sci. Res. M. Sars No. Atl. Deep-sea Exp. 1910, 4, (2), p. 19, pl. 2, fig. D-Atlantic, ca. $57^{\circ}$ N., $11^{\circ}$ W., 1853 meters.

Melanostigma gelatinosum Goode and Bean, 1883, Bull. Mus. Comp. Zool., 10: 209; 1895, Ocean. Ichth., p. 314, fig. 284; Jordan and Evermann, 1898, Bull. U. S. Nat. Mus., 47: 2479; ? Gilchrist and von Bonde, 1924, Rep. Fish. Mar. Biol. Surv. So. Afr., 3, (7), p. 20; ? Barnard, 1927, Ann. So. Afr. Mus., 21 : 874; ? Smith, 1949, Sea Fishes So. Afr., p. 365, fig. 1025.

Deep-abyssal records.-Eastern Atlantic, ca. $57^{\circ} \mathrm{N} ., 11^{\circ} \mathrm{W} ., 1853$ meters, one specimen.

(?) Atlantic off South Africa, ca. $33^{\circ}$ S., $16^{\circ}$ E., 2561 meters, one specimen. 
Distribution.--Eastern Atlantic, one deep-abyssal record. Western Atlantic off the United States coast, $37^{\circ}-39^{\circ}$ N., an unstated number of specimens from eleven stations in 723-1172 meters. (?) South Africa, one deep-abyssal record.

Length. $-86-140 \mathrm{~mm}$.

\section{Pachycara obesa Zugmayer}

Pachycara obesa Zugmayer, 1911, Bull. Inst. Océanogr. Monaco, 193: 12 -Gulf of Gascony, ca. $45^{\circ} \mathrm{N} ., 6^{\circ} \mathrm{W}$.

Pachycara obesa Zugmayer, 1911, Rés. Camp. Sci. Monaco, 35: 134, pl. 6, fig. 6; Fowler, 1936, Bull. Amer. Mus. Nat. Hist., 70: 1056; Belloc, 1949, Bull. Inst. Océanogr. Monaco, 958: 13, pl. 3.

Pachycarichthys obesa Whitley, 1931, Australian Zool., 6: 334.

Deep-abyssal record and distribution.-Eastern Atlantic, 4780 meters, one specimen.

Length. $-385 \mathrm{~mm}$.

\section{Family LYCODAPODIDAE}

The family, with only one genus, contains ten species ranging from the Okhotsk Sea across the north Pacific and south to the Gulf of Panama, and one species from the Strait of Magellan. Except for $L$. fierasfer all of these fishes are archibenthic.

\section{Lycodapus fierasfer Gilbert}

Lycodapus fierasfer Gilbert, 1890, Proc. U. S. Nat. Mus., 13: 108-Pacific off United States coast and in the Gulf of California (type locality not stated).

Lycodapus fierasfer Gilbert, 1896, Rep. U. S. Comm. Fish, 1893: 455, 471, pl. 32; Jordan and Evermann, 1898, Bull. U. S. Nat. Mus., 47: 2493; Garman, 1899, Mem. Mus. Comp. Zool., 24: 139; Evermann and Goldsborough, 1907, Bull. U. S. Bur. Fish., 26: 345; Townsend and Nichols, 1925, Bull. Amer. Mus. Nat. Hist., 52: 16; Hubbs and Schultz, 1932, Contr. Can. Biol. Fish., 7: 323; Clemens and Wilby, 1946, Bull. Fish. Res. Bd. Canada, 68: 195, fig. 132; Böhlke, 1953, Stanf. Ichth. Bull., 5: 101.

Deep-abyssal records.-Eastern Pacific, ca. $31^{\circ}$ N., $118^{\circ}$ W., 1968 meters, one specimen.

Eastern Pacific in the Gulf of California, 1838 meters, (?) specimens.

Distribution.-Eastern and north Pacific from Bering Sea to the Gulf of Panama. Depth range between 111-120 and 640 meters south to British Columbia; one deep-abyssal record and in 6981103 meters off the United States coast; one deep-abyssal record 
in the Gulf of California; and in 1644 meters in the Gulf of Panama. More than sixteen specimens reported.

Length. $-63.5-152 \mathrm{~mm}$.

\section{Family DEREPODICHTHYIDAE}

The family contains only one species.

\section{Derepodichthys alepidotus Gilbert}

Derepodichthys alepidotus Gilbert, 1896, Rep. U. S. Comm. Fish, 1893: 456 -Pacific off the Queen Charlotte Islands.

Derepodichthys alepidotus Jordan and Evermann, 1898, Bull. U. S. Nat. Mus., 47: 2480; Clemens and Wilby, 1946, Bull. Fish. Res. Bd. Canada, 68: 197, fig. 134.

Deep-abyssal record and distribution.-Northeastern Pacific, ca. $52^{\circ} \mathrm{N}$., $132^{\circ} \mathrm{W} ., 2904$ meters, one specimen.

Length. $-110 \mathrm{~mm}$.

\section{Family BROTULIDAE}

Including the eleven found on the abyssal plain, fifty-seven species of brotulid fishes (twenty-four genera) have been reported from deep-abyssal waters. Most of the species live in tropical and temperate latitudes, the only exceptions being one species from Greenland and one Antarctic form, the latter a deep-abyssal one. There are shore and fresh-water, as well as deep-sea forms. Young stages of some oceanic species live pelagically for an undetermined length of time.

Deep-abyssal brotulids do not, in general, show the wide geographical distribution that is seen in some families. Seemingly correlated with this more limited distribution is a restricted bathymetric range. However, there are enough examples of species reported from widespread areas to suggest that future investigations will increase the number. Mixonus caudalis is known from the Indian Ocean and the eastern Pacific and has been taken only once above the deep-abyssal zone (1524 meters) while Acanthonus armatus and Bassozetus compressus have been caught only in deep-abyssal waters but in both the western Pacific and eastern Atlantic.

Pale or colorless and sometimes blind forms often develop under suitable conditions. Several cave-dwelling species fall into this category, as well as a few burrowing shore forms; in deep-abyssal 
waters there are eleven species with rudimentary eyes. In the deep sea above 2000 meters there are only two blind species and of these Aphyonus mollis Goode and Bean may eventually prove to be deepabyssal, having been caught only once, in 1747 meters. The other, Barathronus diaphanus Brauer, came from 918, 1289 and 1756 meters. Although few of these blind, or nearly blind, species have been described from fresh specimens only three show much pigment beyond the black peritoneum and gill-cover membranes.

Monomitopus metriostomus Vaillant has been omitted here, the one deep-abyssal specimen having proved to be a different fish (Nybelin, 1953).

Nybelin $(1951$, pp. 16, 19, 24) reported from deep-abyssal waters in the north Atlantic the following three unnamed brotulids: Porogadus sp., one specimen, 5250-5300 meters (ca. $1^{\circ}$ N., $18^{\circ}$ W.); Barathrites sp., one specimen, 5044-5033 meters (ca. $12^{\circ} \mathrm{N}$., $52^{\circ} \mathrm{W}$.); and Leucochlamys sp., two specimens, 4590-5600 meters (ca. $40^{\circ} \mathrm{N}$., $35^{\circ}$ W. and $9^{\circ}$ N., $26^{\circ}$ W.).

\section{Monomeropus malispinosus Garman}

Monomeropus malispinosus Garman, 1899, Mem. Mus. Comp. Zool., 24: 158, pl. 40, fig. 2-Pacific off Panama, ca. $7^{\circ} \mathrm{N}$., $79^{\circ} \mathrm{W}$.

Deep-abyssal record and distribution.-Eastern Pacific, 1865 meters, one specimen.

Length. $-216 \mathrm{~mm}$.

\section{Dicrolene filamentosa Garman}

Dicrolene filamentosa Garman, 1899, Mem. Mus. Comp. Zool., 24: 149, pl. F, fig. 1, pl. 75, fig. 2-Pacific off Mexico, ca. $16^{\circ}$ N., $99^{\circ}$ W., 1207 meters.

Deep-abyssal record.-Eastern Pacific, ca. $7^{\circ}$ N., $79^{\circ}$ W., 1865 meters, (?) specimens.

Distribution.-Eastern Pacific off Mexico and in the Gulf of Panama, one deep-abyssal record and an unstated number of specimens from three hauls in 935-1207 meters.

Length. - $--432 \mathrm{~mm}$.

\section{Dicrolene nigra Garman}

Dicrolene nigra Garman, 1899, Mem. Mus. Comp. Zool., 24: 150, pl. 37, fig. 2 , pl. 75 , fig. 1 -Pacific off the Galapagos Islands, ca. $0^{\circ} 57^{\prime} \mathrm{S} ., 89^{\circ} \mathrm{W}$., 769 meters.

Dicrolene nigra Trotter, 1926, Zoologica, 8: 114; Gregory, 1933, Trans. Amer. Phil. Soc., 23, (2), p. 378, fig. 256. 
Deep-abyssal record.-Eastern Pacific, ca. $7^{\circ}$ N., $79^{\circ}$ W., 1865 meters, (?) specimens.

Distribution.-Eastern Pacific off Panama, Cocos Island, and the Galapagos Islands, at least thirty-seven specimens known, one deep-abyssal record, others in 769-1644 meters. Probably accidental in the deep-abyssal zone.

Length. $-125-481 \mathrm{~mm}$.

Remarks.--In one haul made at a depth of 1143 meters near Cocos Island, thirty-one specimens were caught, only six of them adult.

Barathrodemus manatinus Goode and Bean

Barathrodemus manatinus Goode and Bean, 1883, Bull. Mus. Comp. Zool., $10: 200$-Atlantic off United States coast, ca. $33^{\circ} \mathrm{N} ., 76^{\circ} \mathrm{W} ., 1183$ meters.

Barathrodemus manatinus Günther, 1887, Rep. Sci. Res. Voy. Challenger, Zool., 22: 100; Goode and Bean, 1895, Ocean. Ichth., p. 332, fig. 294; Jordan and Evermann, 1898, Bull. U. S. Nat. Mus., 47: 2517; 1900, op. cit., fig. 880.

Deep-abyssal record.-Western Atlantic, ca. $37^{\circ}$ N., $73^{\circ}$ W., 2552 meters, (?) specimens.

Distribution.-Western Atlantic, $33^{\circ}-37^{\circ}$ N., $73^{\circ}-76^{\circ}$ W., one deep-abyssal record and at least three specimens from two hauls in 1624 and 1183 meters.

Length.-?-159 $\mathrm{mm}$.

\section{Barathrodemus nasutus Smith and Radcliffe}

Barathrodemus nasutus Smith and Radcliffe, in Radcliffe, 1913, Proc. U. S. Nat. Mus., 44: 152, pl. 10, fig. 2-Gulf of Tomini, Celebes.

Barathrodemus nasutus Cockerell, 1916, Ann. Mag. Nat. Hist., (8), 18: 323; de Beaufort and Chapman, 1951, Fishes Indo-Austr. Arch., 9: 425, fig. 71.

Deep-abyssal records and distribution.-Western Pacific, Gulf of Tomini, Celebes, Dutch East Indies, 1997, 1992 meters, three specimens from two hauls.

Length.-?-172 mm.

\section{Barathrites iris Zugmayer}

Barathrites iris Zugmayer, 1911, Bull. Inst. Océanogr. Monaco, 193: 11 -Atlantic, ca. $31^{\circ} \mathrm{N} ., 42^{\circ} \mathrm{W} ., 3465$ meters.

Barathrites iris Zugmayer, 1911, Rés. Camp. Sci. Monaco, 35: 132, pl. 6, fig. 3; Parr, 1933, Bull. Bingham Oceanogr. Coll., 3, (6), p. 46; Fowler, 1936, Bull. Amer. Mus. Nat. Hist., 70: 1063, fig. 442; Belloc, 1949, Bull. Inst. Océanogr. Monaco, 958: 14, pl. 3. 
Deep-abyssal record.-Mid-Atlantic on or west of the midAtlantic ridge, ca. $31^{\circ}$ N., $42^{\circ}$ W., 3465 meters, one specimen.

Distribution.-Mid-Atlantic, one deep-abyssal record. Western Atlantic near the Bahamas, one specimen in 1645-1747 meters.

Length. - 233-250 mm.

\section{Barathrites abyssorum Roule}

Barathrites abyssorum Roule, 1916, Bull. Inst. Océanogr. Monaco, 320: 17 -Atlantic, ca. $30^{\circ} \mathrm{N} ., 25^{\circ} \mathrm{W}$.

Barathrites Roule, 1915, C. R. Acad. Sci. Paris, 161 : 56.

Barathrites abyssorum Roule, 1919, Rés. Camp. Sci. Monaco, 52: 71, pl. 2, fig. 3 ; 1934, Poiss. Monde Viv. Eaux, $7: 191-192$, pl. 11, text fig.; Belloc, 1949, Bull. Inst. Océanogr. Monaco, 958: 14.

Deep-abyssal record and distribution.-Eastern Atlantic, 5285 meters, one specimen.

Length. $-260 \mathrm{~mm}$.

\section{Bassogigas crassus Vaillant}

Bythites crassus Vaillant, 1888, Exp. Sci. Trav. Talis., Poiss., p. 279, pl. 25, fig. 1-Atlantic between France and the Azores (see footnote, p. 110).

Neobythites crassus Goode and Bean, 1895, Ocean. Ichth., p. 327; Koefoed, 1927, Rep. Sci. Res. M. Sars No. Atl. Deep-sea Exp. 1910, 4, (1), p. 133, pl. 6, fig. 1; Fowler, 1936, Bull. Amer. Mus. Nat. Hist., 70: 1064, fig. 443.

Bassogigas crassus Nybelin, 1948, Göteborgs K. Vetensk. Vitt.-Samh. Handl., (B), 5, (16), p. 76, pl. 5, figs. 1-3, pl. 6, figs. 1-3.

Deep-abyssal records.-Eastern Atlantic, 4255 meters, the type.

Mid-Atlantic on the mid-Atlantic ridge, ca. $45^{\circ} \mathrm{N}$., $25^{\circ} \mathrm{W}$, 3120 meters, one specimen.

Eastern Atlantic, ca. $35^{\circ}$ N., $8^{\circ}$ W., 2150-2300 and 2055 meters, two specimens.

Distribution.-Eastern and mid-Atlantic, four deep-abyssal records and eleven specimens in one haul in 1797 meters (ca. $50^{\circ} \mathrm{N}$., $11^{\circ}$ W.). Probably a deep-abyssal species.

Length. $-300-550 \mathrm{~mm}$.

\section{Bassogigas pterotus Alcock}

Neobythites pterotus Alcock, 1890, Ann. Mag. Nat. Hist., (6), 6: 210, 297 -Bay of Bengal, ca. $18^{\circ}$ N., $85^{\circ}$ E., 2395 meters.

Neobythites pterotus Alcock, 1891, op. cit., (6), 7:11; 1891, op. cit., (6), 8: $30 ; 1894$, Ill. Zool. Investigator, Fishes, pl. 11, fig. 4; Alcock and McArdle, 1900 , op. cit., pl. 29 , fig. 1 . 
Bassogigas pterotus Goode and Bean, 1895, Ocean. Ichth., pp. 328, 529; Smith and Radcliffe, in Radcliffe, 1913, Proc. U. S. Nat. Mus., 44: 154; Norman, 1939, Sci. Rep. John Murray Exp. 1933-34, 7, (1), p. 86.

Neobythites (Bassogigas) pterotus Alcock, 1899, Descr. Cat. Indian Deep-sea Fishes, p. 83.

Deep-abyssal records and distribution.-North Indian Ocean, ca. $11^{\circ}$ N., $88^{\circ}$ E., 3197 meters, one specimen.

North Indian Ocean, 2395 meters, the type.

North Indian Ocean, ca. $11^{\circ} \mathrm{N} ., 74^{\circ}$ E., 1829 meters, one specimen.

Probably a deep-abyssal species.

Length.-Ca. 209-300 mm.

\section{Bassogigas aequatoris Smith and Radcliffe}

Bassogigas aequatoris Smith and Radcliffe, in Radcliffe, 1913, Proc. U. S. Nat. Mus., 44: 153, pl. 10, fig. 3-Pacific, Gulf of Tomini, Celebes.

Bassogigas aequatoris Cockerell, 1916, Ann. Mag. Nat. Hist., (8), 18: 324; de Beaufort and Chapman, 1951, Fishes Indo-Austr. Arch., 9: 326, fig. 72.

Deep-abyssal record and distribution.-Western Pacific, 1992 meters, one specimen.

Length. $-288 \mathrm{~mm}$.

\section{Bassogigas digittatus Garman}

Holcomycteronus digittatus Garman, 1899, Mem. Mus. Comp. Zool., 24: 163, pl. 36, fig. 1, pl. 76, fig. 2-eastern Pacific from the Gulf of California to off northern South America (type locality not designated).

Bassogigas digittatus Norman, 1939, Sci. Rep. John Murray Exp. 1933-34, 7, (1), p. 86.

Deep-abyssal records and distribution.-Ca. $10^{\circ}$ N., $96^{\circ}$ W., 4082 meters, (?) specimens.

Ca. $14^{\circ}$ N., $98^{\circ} \mathrm{W} ., 3436$ meters, (?) specimens.

Ca. $2^{\circ}$ N., $83^{\circ}$ W., 3333 meters, (?) specimens.

Ca. $25^{\circ}$ N., $109^{\circ}$ W., 2904 meters, (?) specimens.

Ca. $2^{\circ}$ N., $82^{\circ}$ W., 2196 meters, (?) specimens.

Eastern Pacific only, $2^{\circ}-25^{\circ}$ N., $82^{\circ}-109^{\circ} \mathrm{W}$. Probably chiefly or exclusively deep-abyssal and also reaching the abyssal plain.

Length.--?-356 mm.

\section{Bassogigas koefoedi Nybelin}

Bassogigas koefoedi Nybelin, 1953, 14 Intern. Zool. Congr. Copenhagen, p. 2 -Atlantic, ca. $27^{\circ} \mathrm{N} ., 14^{\circ} \mathrm{W}$. 
Bassogigas (Holcomycteronus) digittatus Koefoed, 1927, Rep. Sci. Res. M. Sars

No. Atl. Deep-sea Exp. 1910, 4, (1), p. 140, pl. 6, fig. 2.

Deep-abyssal record and distribution.-Eastern Atlantic, 2603 meters, one specimen.

Length. $-310 \mathrm{~mm}$.

\section{Bassogigas brucei Dollo}

Neobythites brucei Dollo, 1905-06, Proc. Roy. Soc. Edinburgh, 26: 172 -Antarctic, ca. $67^{\circ} \mathrm{S} ., 36^{\circ} \mathrm{W}$.

Neobythitcs brucei Regan, 1913, Trans. Roy. Soc. Edinburgh, 49: 238, pl. 3, fig. 2.

Holcomycteronus brucei Regan, 1914, Brit. Antarctic (Terra Nova) Exp. 1910, Zool., 1 : 39.

Bassogigas brucei Norman, 1939, Sci. Rep. John Murray Exp. 1933-34, 7, (1), p. 86.

Deep-abyssal record and distribution.-Antarctic, Weddell Sea, 4571 meters, one specimen.

Length. $-350 \mathrm{~mm}$.

\section{Bassogigas grandis Günther}

Sirembo grandis Günther, 1877, Ann. Mag. Nat. Hist., (4), 20 : 437-Pacific off Japan, ca. $34^{\circ} \mathrm{N}$, $140^{\circ} \mathrm{E}$.

Neobythites grandis Günther, 1887, Rep. Sci. Res. Voy. Challenger, Zool., 22: 100 , pl. 21, fig. A.

Bassogigas grandis Goode and Bean, 1895, Ocean. Ichth., p. 529; Tanaka, 1908, Jour. Coll. Sci. Imp. Univ. Tokyo, 23, (13), p. 17, pl. 2, fig. 1.

Deep-abyssal record.-Western Pacific, 3465 meters, the type.

Distribution.-Western Pacific off Japan, one deep-abyssal record and one specimen from an unknown depth.

Length. $-750,1250 \mathrm{~mm}$.

\section{Bassogigas gilli Goode and Bean}

Bassogigas gilli Goode and Bean, 1895, Ocean. Ichth., pp. 328, 529, fig. 291 -Atlantic off United States coast, ca. $39^{\circ}$ N., $70^{\circ} \mathrm{W}$.

Bassogigas gilli Jordan and Evermann, 1898, Bull. U. S. Nat. Mus., 47: $2515 ; 1900$, op. cit., fig. 879 .

Deep-abyssal record and distribution.-Western Atlantic, 2022 meters, one specimen.

Length. $-463 \mathrm{~mm}$.

\section{Alcockia rostrata Günther}

Porogadus rostratus Günther, 1887, Rep. Sci. Res. Voy. Challenger, Zool., 22: 113, pl. 4, fig. B Pacific north of Celebes, ca. $2^{\circ}$ N., $124^{\circ} \mathrm{E}$. 
Alcockia rostrata Goode and Bean, 1895, Ocean. Ichth., p. 329; Norman, 1939,

Sci. Rep. John Murray Exp. 1933-34, 7, (1), p. 87; de Beaufort and Chapman, 1951, Fishes Indo-Austr. Arch., 9: 429, fig. 74.

Deep-abyssal record and distribution.-Western Pacific, 3932 meters, one specimen.

Length. $-292 \mathrm{~mm}$.

\section{Bassozetus normalis Gill}

Bassozetus normalis Gill, 1883, Proc. U. S. Nat. Mus., 6: 259-Atlantic off United States coast, ca. $39^{\circ} \mathrm{N} ., 68^{\circ} \mathrm{W}$., 2844 meters.

Bassozetus normalis Günther, 1887, Rep. Sci. Res. Voy. Challenger, Zool., 22: 99; Goode and Bean, 1895, Ocean. Ichth., p. 322, fig. 287; Jordan and

Evermann, 1898, Bull. U. S. Nat. Mus., 47: 2507; 1900, op. cit., fig. 875.

Deep-abyssal records and distribution.-Western Atlantic, ca. $24^{\circ}$ N., $84^{\circ}$ W., 3512 meters, (?) specimens.

Western Atlantic, ca. $39^{\circ}$ N., $68^{\circ}$ W., 2844 meters, (?) specimens.

Western Atlantic, ca. $28^{\circ} \mathrm{N}$., $87^{\circ} \mathrm{W}$., 2615 meters, (?) specimens.

Western Atlantic, Gulf of Mexico, 2104-2194 meters, two specimens (Oregon Station $1303,28^{\circ} 47^{\prime}$ N., $87^{\circ} 50^{\prime}$ W., May 26, 1955).

Western Atlantic, ca. $16^{\circ}$ N., $61^{\circ}$ W., 2068 meters, (?) specimens.

Western Atlantic only, from the Lesser Antilles, the Gulf of Mexico, and off New Jersey. Exclusively deep-abyssal as far as known.

Length. $-170-329 \mathrm{~mm}$.

\section{Bassozetus taenia Günther}

Bathyonus taenia Günther, 1887, Rep. Sci. Res. Voy. Challenger, Zool., 22: 110 , pl. 23 , fig. A-Atlantic, ca. $2^{\circ} \mathrm{N} ., 20^{\circ} \mathrm{W}$.

Bassozetus taenia Goode and Bean, 1895, Ocean. Ichth., p. 323; Jordan and Evermann, 1898, Bull. U. S. Nat. Mus., 47: 2510; Roule, 1935, Bull. Inst. Océanogr. Monaco, 674: 6; Fowler, 1936, Bull. Amer. Mus. Nat. Hist., 70: 1062, fig. 441; Nybelin, 1953, 14 Intern. Zool. Congr. Copenhagen, p. 3 .

Bassozetus sp. Nybelin, 1951, Rep. Swedish Deep-sea Exp. 1947-1948, Zool., 2, (1), pp. 15, 19.

Deep-abyssal records and distribution.-Mid-Atlantic east of the mid-Atlantic ridge, ca. $9^{\circ} \mathrm{N} ., 26^{\circ} \mathrm{W}$., $5610-5600$ meters, one specimen.

Western Atlantic, ca. $12^{\circ}$ N., $52^{\circ}$ W., 5044-5033 meters, two specimens.

Mid-Atlantic east of the mid-Atlantic ridge, 4571 meters, the type. 
Known so far only from the abyssal plain.

Length.-?-254 mm.

Remarks.--Roule (1935, p. 6) reported this species from Madeira, but without description or depth data.

\section{Bassozetus nasus Garman}

Bassozetus nasus Garman, 1899, Mem. Mus. Comp. Zool., 24: 159, 361, pls. 77, 78-Pacific off Central America (type locality not designated).

Bassozetus nasus Lendenfeld, 1905, op. cit., 30 : 197, pl. 4, figs. 14-17; Trojan, 1906 , op. cit., p. 245 , pls. 5,6 .

Deep-abyssal records and distribution.-Eastern Pacific, $4^{\circ}-14^{\circ}$ N., $80^{\circ}-98^{\circ}$ W., $3436,3240,3057$ meters, an unstated number of specimens from three hauls.

Length.-?-279 $\mathrm{mm}$.

\section{Bassozetus compressus Günther}

Bathynectes compressus Günther, 1878, Ann. Mag. Nat. Hist., (5), 2: 20 -type locality not stated, designated as Challenger Station 184, ca. $12^{\circ}$ S., $145^{\circ}$ E., 2560 meters, the locality of the specimen figured by Günther, 1887.

Bathyonus compressus Günther, 1887, Rep. Sci. Res. Voy. Challenger, Zool., 22: 109, pl. 22, fig. A.

Bassozetus compressus Goode and Bean, 1895, Ocean. Ichth., p. 322; Jordan and Evermann, 1898, Bull. U. S. Nat. Mus., 47: 2508; Radcliffe, 1913, Proc. U. S. Nat. Mus., 44: 157; Fowler, 1936, Bull. Amer. Mus. Nat. Hist., 70: 1062; de Beaufort and Chapman, 1951, Fishes Indo-Austr. Arch., 9: 431.

Deep-abyssal records and distribution.-Mid-Atlantic east of the mid-Atlantic ridge, ca. $1^{\circ} \mathrm{N} ., 26^{\circ} \mathrm{W} ., 2744$ meters, one specimen.

Western Pacific south of New Guinea, 2560 meters, two specimens.

Western Pacific off the Philippines, ca. $16^{\circ}$ N., $119^{\circ}$ E., 1920 meters, one specimen.

Length. - $114-432 \mathrm{~mm}$.

\section{Bassozetus elongatus Smith and Radcliffe}

Bassozetus elongatus Smith and Radcliffe, in Radcliffe, 1913, Proc. U. S. Nat. Mus., 44: 157, pl. 11, fig. 4-Gulf of Tomini, Celebes.

Bassozetus elongatus Cockerell, 1916, Ann. Mag. Nat. Hist., (8), 18: 322; de Beaufort and Chapman, 1951, Fishes Indo-Austr. Arch., 9: 433, fig. 75; Böhlke, 1953, Stanf. Ichth. Bull., 5: 102. 
Deep-abyssal record and distribution.-Western Pacific, 1992 meters, two(?) specimens.

Length. $-480 \mathrm{~mm}$.

\section{Bassozetus glutinosus Alcock}

Bathyonus glutinosus Alcock, 1890, Ann. Mag. Nat. Hist., (6), 6: 211-Bay of Bengal, ca. $18^{\circ} \mathrm{N} ., 85^{\circ}$ E., 2395 meters.

Bathyonus glutinosus Alcock, 1891, op. cit., (6), $7: 11$; 1892, Ill. Zool. Investigator, Fishes, pl. 1, fig. 3.

Bassozetus glutinosus Goode and Bean, 1895, Ocean. Ichth., p. 322; Alcock, 1899, Descr. Cat. Indian Deep-sea Fishes, p. 88; Norman, 1939, Sci. Rep. John Murray Exp. 1933-34, 7, (1), p. 77.

Bathyonus (Bassozetus) glutinosus McArdle, 1901, Ann. Mag. Nat. Hist., (7), $8: 518$.

Deep-abyssal records.-North Indian Ocean, ca. $18^{\circ}$ N., $85^{\circ}$ E., 2395 meters, five specimens.

North Indian Ocean, Gulf of Aden, 2312 meters, one specimen.

North Indian Ocean, ca. $10^{\circ}$ N., $74^{\circ}$ E., 2104-2140 meters, (?) specimens.

North Indian Ocean, ca. $7^{\circ}$ N., $76^{\circ}$ E., 1840 meters, (?) specimens.

Distribution.-North Indian Ocean in the Bay of Bengal and the Arabian Sea, four deep-abyssal records and an unstated number of specimens from three hauls in 1163-1629 meters.

Length. $-178-270 \mathrm{~mm}$.

\section{Bassozetus oncerocephalus Vaillant}

Sirembo oncerocephalus Vaillant, 1888, Exp. Sci. Trav. Talis., Poiss., pp. 277, 387, pl. 24, fig. 6-Atlantic off Cape Verde (see footnote, p. 110).

Dicromita oncerocephala Goode and Bean, 1895, Ocean Ichth., p. 321; Fowler, 1925, Amer. Mus. Nov., 162: 5 (subgenus Pterodicromita); 1936, Bull. Amer. Mus. Nat. Hist., 70: 1060, fig. 440.

Monomitopus oncerocephalus Fowler, 1936, op. cit., p. 1329.

[Bassozetus] oncerocephalus Norman, 1929, Sci. Rep. John Murray Exp. 1933$34,7,(1)$, p. 87.

Deep-abyssal record and distribution.-Eastern Atlantic, 3200 meters, one specimen.

Length.- $-221 \mathrm{~mm}$.

\section{Eretmichthys pinnatus Garman}

Eretmichthys pinnatus Garman, 1899, Mem. Mus. Comp. Zool., 24: 165, 362, pl. 35, figs. 1-4, pl. 79, fig. 2-Pacific off Panama and Ecuador (type locality not designated). 
Deep-abyssal records.-Eastern Pacific, ca. $0^{\circ} 36^{\prime}$ S., $86^{\circ} \mathrm{W}$., 2417 meters, (?) specimens.

Eastern Pacific, ca. $5^{\circ}$ N., $86^{\circ}$ W., 1951 meters, (?) specimens.

Distribution.-Eastern Pacific, ca. $0^{\circ} 36^{\prime}$ S., $86^{\circ} \mathrm{W}$. and ca. $5^{\circ}$ N., $85^{\circ}-86^{\circ} \mathrm{W}$., two deep-abyssal records and an unstated number of specimens from one haul in 1789 meters.

Length.-?-432 $\mathrm{mm}$.

\section{Eretmichthys ocella Garman}

Eretmichthys ocella Garman, 1899, Mem. Mus. Comp. Zool., 24: 166, 362, pl. 37, fig. 1, pl. 79, fig. 1 -Pacific off Panama, ca. $7^{\circ} \mathrm{N}$., $79^{\circ} \mathrm{W}$.

Deep-abyssal record and distribution.-Eastern Pacific off Panama, 2322 meters, one specimen.

Length. $-381 \mathrm{~mm}$.

\section{Eretmichthys remifer Smith and Radcliffe}

Eretmichthys remifer Smith and Radcliffe, in Radcliffe, 1913, Proc. U. S. Nat. Mus., 44: 155, pl. 11, fig. 2-Gulf of Tomini, Celebes.

Eretmichthys remifer Cockerell, 1916, Ann. Mag. Nat. Hist., (8), 18: 322; de

Beaufort and Chapman, 1951, Fishes Indo-Austr. Arch., 9: 428, fig. 73.

Deep-abyssal record and distribution.-Western Pacific, 1997 meters, one specimen.

Length. $-272 \mathrm{~mm}$.

\section{Porogadus miles Goode and Bean}

Porogadus miles Goode and Bean, 1885, Proc. U. S. Nat. Mus., 8: 602Atlantic off coast of United States, ca. $38^{\circ} \mathrm{N} ., 73^{\circ} \mathrm{W}$.

Porogadus miles Günther, 1887, Rep. Sci. Res. Voy. Challenger, Zool., 22: 113; Goode and Bean, 1895, Ocean. Ichth., p. 334, fig. 292; Jordan and

Evermann, 1898, Bull. U. S. Nat. Mus., 47: 2520; 1900, op. cit., fig. 881; Gilchrist, 1906, Mar. Invest. So. Afr., 4: 159; Thompson, *1916, Prov. Cape Good Hope Mar. Biol. Rep., 3: 107; Barnard, 1927, Ann. So. Afr. Mus., 21: 881; Smith, 1949, Sea Fishes So. Afr., p. 364, fig. 1022.

Deep-abyssal records.-Western Atlantic, 2136 meters, the type.

Western Atlantic, Gulf of Mexico, 2104-2194 meters, one specimen (Oregon Station 1303, $28^{\circ} 47^{\prime}$ N., $87^{\circ} 50^{\prime}$ W., May 26, 1955).

Distribution.-Western Atlantic off the northern coast of the United States and in the Gulf of Mexico, two deep-abyssal specimens. South Africa off Cape Point, one specimen in 1280-1463 meters.

Length.-153-291 mm. 


\section{Porogadus nudus Vaillant}

Porogadus nudus Vaillant, 1888, Exp. Sci. Trav. Talis., Poiss., p. 262, pl. 24, fig. 2-Atlantic off North Africa, 2324 meters (see footnote, p. 110).

Celema nuda Goode and Bean, 1895, Ocean. Ichth., p. 330; Fowler, 1936, Bull. Amer. Mus. Nat. Hist., 70: 1067, fig. 446.

Porogadus nudus Brauer, 1906, Wiss. Ergebn. Deutschen Tiefsee Exp. Valdivia, 15, (1), p. 404; Norman, 1939, Sci. Rep. John Murray Exp. 1933-34, 7, (1), p. 87.

Deep-abyssal records and distribution.-Eastern Atlantic off Cape Verde, 3200 meters, one specimen, a doubtful identification.

Eastern Atlantic, Banc d'Arguin (North Africa), 2324, meters, three specimens.

Length.-?-202 mm.

\section{Porogadus catena Goode and Bean}

Bathyonus catena Goode and Bean, 1885, Proc. U. S. Nat. Mus., 8: 603-Gulf of Mexico, ca. $28^{\circ}$ N., $87^{\circ} \mathrm{W}$.

Bathyonus catena Günther, 1887, Rep. Sci. Res. Voy. Challenger, Zool., 22: 111.

Bassozetus catena Goode and Bean, 1895, Ocean. Ichth., p. 323, fig. 286; Jordan and Evermann, 1898, Bull. U. S. Nat. Mus., 47: 2509; 1900, op. cit., figs. $876,876, a$.

Porogadus catena Norman, 1939, Sci. Rep. John Murray Exp. 1933-34, 7, (1), p. 87.

Deep-abyssal record and distribution.-Western Atlantic in the Gulf of Mexico, 2683 meters, two specimens.

Length. -227-237 mm.

\section{Porogadus gracilis Günther}

Bathynectes gracilis Günther, 1878, Ann. Mag. Nat. Hist., (5), 2: 21-Pacific south of New Guinea, ca. $12^{\circ} \mathrm{S} ., 145^{\circ} \mathrm{E}$.

Porogadus gracilis Günther, 1887, Rep. Sci. Res. Voy. Challenger, Zool., 22 : 112, pl. 16, fig. B; Brauer, 1906, Wiss. Ergebn. Deutschen Tiefsee Exp. Valdivia, 15, (1), p. 404; Norman, 1939, Sci. Rep. John Murray Exp. 1933-34, 7, (1), p. 87.

Moebia gracilis Goode and Bean, 1895, Ocean. Ichth., p. 331; de Beaufort and Chapman, 1951, Fishes Indo-Austr. Arch., 9: 436, fig. 77.

Deep-abyssal record and distribution.-Western Pacific, 2560 meters, one specimen.

Length. $-229 \mathrm{~mm}$. 


\section{Porogadus trichiurus Alcock}

Dermatorus trichiurus Alcock, 1890, Ann. Mag. Nat. Hist., (6), 6: 298Arabian Sea, ca. $11^{\circ}$ N., $74^{\circ}$ E., 1829 meters.

Dermatorus trichiurus Alcock, 1891, op. cit., (6), 7: 11; 1892, Ill. Zool. Investigator, Fishes, pl. 1, fig. 1; 1899, Descr. Cat. Indian Deep-sea Fishes, p. 90; Goode and Bean, 1895, Ocean. Ichth., p. 325; McArdle, 1901, Ann. Mag. Nat. Hist., (7), 8: 518.

Porogadus trichiurus Norman, 1939, Sci. Rep. John Murray Exp. 1933-34, 7, (1), p. 77.

Deep-abyssal records.-Indian Ocean, Gulf of Aden, 2312 meters, two specimens.

Arabian Sea, ca. $10^{\circ}$ N., $74^{\circ}$ E., 2104-2140 meters, (?) specimens.

Arabian Sea, $7^{\circ}-11^{\circ}$ N., $74^{\circ}-76^{\circ}$ E., $1893,1840,1829$ meters, two specimens from two hauls and an unstated number from a third haul.

Distribution.-North Indian Ocean in the Gulf of Aden, the Arabian Sea, and near Zanzibar; five deep-abyssal records, one specimen in 1789 meters and an unstated number from two hauls in 1628 and $1336-1410$ meters.

Length. $-130-180 \mathrm{~mm}$.

\section{Porogadus melanocephalus Alcock}

Dermatorus melanocephalus Alcock, 1891, Ann. Mag. Nat. Hist., (6), 8: 32 -Bay of Bengal, ca. $12^{\circ}$ N., $90^{\circ}$ E., 3006 meters.

Dermatorus melanocephalus Goode and Bean, 1895, Ocean. Ichth., p. 325; Alcock and Anderson, 1898, Ill. Zool. Investigator, Fishes, pl. 21, fig. 4; Alcock, 1899, Descr. Cat. Indian Deep-sea Fishes, p. 91.

Porogadus melanocephalus Norman, 1939, Sci. Rep. John Murray Exp. 1933$34,7,(1)$, p. 87.

Deep-abyssal records and distribution.-North Indian Ocean, $11^{\circ}-12^{\circ}$ N., $88^{\circ}-90^{\circ}$ E., 3197,3006 meters, two specimens.

Length.-?-203 $\mathrm{mm}$.

\section{Porogadus subarmatus Vaillant}

Porogadus subarmatus Vaillant, 1888, Exp. Sci. Trav. Talis., Poiss., p. 265, pl. 24, fig. 3-Atlantic off Cape Verde, 3200 meters (see footnote, p. 110).

Celema subarmata Goode and Bean, 1895, Ocean. Ichth., p. 330; Fowler, 1936, Bull. Amer. Mus. Nat. Hist., 70: 1068.

Porogadus subarmatus Brauer, 1906, Wiss. Ergebn. Deutschen Tiefsee Exp. Valdivia, 15, (1), p. 404; Norman, 1939, Sci. Rep. John Murray Exp. 1933-34, 7, (1), p. 87. 
Deep-abyssal records and distribution.--Eastern Atlantic, 3200 meters, eleven specimens from one haul.

Western Atlantic, Gulf of Mexico, 2104-2194 meters, two specimens (Oregon Station 1303, $28^{\circ} 47^{\prime}$ N., 87 $50^{\prime}$ W., May 26, 1955).

Length.-176-222+ $\mathrm{mm}$.

Remarks.-The type of the species was a ripe female, suggesting that the fishes had congregated for spawning, as mentioned by Goode and Bean (1895, p. 330). It is somewhat amazing that from this same haul the Talisman collected four other brotulid species, three of them new (one Porogadus nudus, three P. microphthalmus, two Mixonus laticeps and one Bassozetus oncerocephalus). In color they all range from pure white to rosy white, except for the black peritoneum and opercular linings.

\section{Porogadus longiceps Garman}

Porogadus longiceps Garman, 1899, Mem. Mus. Comp. Zool., 24: 153, 361, pl. F, fig. 2, pl. 76, fig. $1-$ Pacific off Colombia and Panama, $4^{\circ}-7^{\circ} \mathrm{N}$, $79^{\circ}-86^{\circ} \mathrm{W}$. (type locality not designated).

Deep-abyssal records.-Eastern Pacific, 3279, 3240, 1865 meters, an unstated number of specimens from three hauls.

Distribution.--Eastern Pacific, three deep-abyssal records and one very young specimen in 245 meters.

Length. - 140-495 mm.

\section{Porogadus atripectus Garman}

Porogadus atripectus Garman, 1899, Mem. Mus. Comp. Zool., 24: 154, pl. 37, fig. 3-Pacific off Mexico and Central America (type locality not designated).

Deep-abyssal records.-Eastern Pacific, ca. $7^{\circ}$ N., $79^{\circ}$ W., 2322 meters, (?) specimens.

Eastern Pacific, ca. $5^{\circ}$ N., 86 $6^{\circ}$ W., 1951 meters, (?) specimens.

Distribution.-Eastern Pacific, two deep-abyssal records and an unstated number of specimens from one haul in 1412 meters (ca. $\left.16^{\circ} \mathrm{N} ., 100^{\circ} \mathrm{W}.\right)$.

Length.--?-318 $\mathrm{mm}$.

\section{Porogadus promelas Gilbert}

Porogadus promelas Gilbert, 1891, Proc. U. S. Nat. Mus., 14: 546-Gulf of California, ca. $27^{\circ} \mathrm{N} ., 111^{\circ} \mathrm{W} ., 1839$ meters.

Moebia promelas Jordan and Evermann, 1898, Bull. U. S. Nat. Mus., 47: 2511; Townsend and Nichols, 1925, Bull. Amer. Mus. Nat. Hist., 52: 16. 
Porogadus promelas Norman, 1939, Sci. Rep. John Murray Exp. 1933-34, 7, (1), p. 87; Böhlke, 1953, Stanf. Ichth. Bull., 5: 102.

Deep-abyssal records.-Eastern Pacific off Lower California, ca. $31^{\circ} \mathrm{N} ., 118^{\circ} \mathrm{W} ., 1968$ meters, one specimen.

Eastern Pacific, Gulf of California, 1839 meters, five specimens.

Distribution.-Eastern Pacific off Lower California, $25^{\circ}-31^{\circ} \mathrm{N}$, and in the Gulf of California, two deep-abyssal records and two specimens in 1607 and 1180 meters.

Length.-?-241 mm.

\section{Penopus macdonaldi Goode and Bean}

Penopus macdonaldi Goode and Bean, 1895, Ocean. Ichth., p. 336, fig. 293 -Atlantic off coast of United States, ca. $38^{\circ} \mathrm{N}$., $70^{\circ} \mathrm{W}$.

Penopus macdonaldi Jordan and Evermann, 1898, Bull. U. S. Nat. Mus., 47: 2521.

Porogadus macdonaldi Brauer, 1906, Wiss. Ergebn. Deutschen Tiefsee Exp. Valdivia, 15, (1), p. 404.

[Porogadus] macdonaldi Norman, 1939, Sci. Rep. John Murray Exp. 1933-34, 7, (1), p. 87.

Deep-abyssal record and distribution.-Western Atlantic, 2982 meters, one specimen.

Length. $-315 \mathrm{~mm}$.

\section{Penopus(?) microphthalmus Vaillant}

Sirembo microphthalmus Vaillant, 1888, Exp. Sci. Trav. Talis., Poiss., p. 275, pl. 24, fig. 4-Atlantic off Cape Verde (see footnote, p. 110).

Dicromita microphthalma Goode and Bean, 1895, Ocean. Ichth., p. 320; Fowler, 1936, Bull. Amer. Mus. Nat. Hist., 70: 1060, fig. 439.

Monomitopus microphthalmus Brauer, 1906, Wiss. Ergebn. Deutschen Tiefsee Exp. Valdivia, 15, (1), p. 407; Fowler, 1936, Bull. Amer. Mus. Nat. Hist., 70: 1329 .

[Porogadus] microphthalmus Norman, 1939, Sci. Rep. John Murray Exp. 1933-34, 7, (1), p. 87.

Deep-abyssal record and distribution.-Eastern Atlantic, 3200 meters, three specimens.

Length.-?-142 $\mathrm{mm}$.

Remarks.-Sirembo microphthalmus Vaillant has been placed by later authors in the genera Dicromita, Monomitopus, and, tentatively, Porogadus. Norman $(1939$, p. 88$)$ noted its resemblance to Penopus macdonaldi. The recent discovery in the Gulf of Mexico of a new species closely related to $S$. microphthalmus has shown that these two fishes cannot be assigned to any known brotulid 
genus as currently understood. However, they are so similar to Penopus Goode and Bean, from which they seem to differ essentially only in scalation, that one is led to suspect a possible growth change in the form of the scales. The type and only specimen of Penopus macdonaldi was $315 \mathrm{~mm}$. long, that of $S$. microphthalmus $142 \mathrm{~mm}$. The latter, and the new form from the Gulf, which is $112 \mathrm{~mm}$. long, have minute, round, embedded scales. These scales are not imbricated, but are close together; with growth they might overlap. The scales of $P$. macdonaldi are also very small but are apparently imbricated.

\section{Mixonus laticeps Günther}

Bathynectes laticeps Günther, 1878, Ann. Mag. Nat. Hist., (5), 2: 20-Atlantic, ca. $2^{\circ} \mathrm{N} ., 20^{\circ} \mathrm{W}$.

Mixonus laticeps Günther, 1887, Rep. Sci. Res. Voy. Challenger, Zool., 22: 108, pl. 25, fig. B; Goode and Bean, 1895, Ocean. Ichth., p. 339, fig. 296, $a$; Jordan and Evermann, 1898, Bull. U. S. Nat. Mus., 47: 2523; Koefoed, 1927, Rep. Sci. Res. M. Sars No. Atl. Deep-sea Exp. 1910, 4, (1), p. 133; Fowler, 1936, Bull. Amer. Mus. Nat. Hist., 70: 1070, fig. 448; Beebe, 1937, Zoologica, 22: 206.

Sirembo Guentheri Vaillant, 1888, Exp. Sci. Trav. Talis., Poiss., pp. 268, 386, pl. 24, fig. 5 .

Deep-abyssal records and distribution.-Mid-Atlantic east of the mid-Atlantic ridge, 4571 meters, the type.

Eastern Atlantic off Cape Verde, 3200 meters, two specimens. Eastern Atlantic, ca. $34^{\circ} \mathrm{N}$., $33^{\circ} \mathrm{W}$., 2615 meters, one specimen. Length.-127-170 mm.

Remarks.-Beebe (1937, p. 206) listed from Bermuda a young specimen, $43 \mathrm{~mm}$., taken in a pelagic net at a depth of 1280 meters.

\section{Mixonus caudalis Garman}

Mixonus caudalis Garman, 1899, Mem. Mus. Comp. Zool., 24: 148, pl. 36, fig. 2, pl. 39, fig. 2, pl. 74, fig. 2-Pacific off northern South America and Panama (type locality not designated).

Mixonus caudalis Trojan, 1906, op. cit., 30 : 235, pls. 3, 4; Brauer, 1906, Wiss. Ergebn. Deutschen Tiefsee Exp. Valdivia, 15, (1), p. 309; Trotter, 1926, Zoologica, 8: 113, fig. 30.

Deep-abyssal records.-Eastern Pacific, ca. $0^{\circ} 36^{\prime} \mathrm{S}$., $86^{\circ} \mathrm{W} ., 2417$ meters, (?) specimens.

Eastern Pacific, $3^{\circ}-5^{\circ}$ N., $82^{\circ}-86^{\circ}$ W., $2150,2070,1951$ meters, an unstated number of specimens from three hauls.

North Indian Ocean, Gulf of Aden, 1840 meters, one specimen. 
Distribution.-Eastern Pacific, ca. $0^{\circ} 36^{\prime}$ S., $86^{\circ} \mathrm{W}$. and $3^{\circ}-5^{\circ}$ N., $82^{\circ}-86^{\circ} \mathrm{W}$., four deep-abyssal records and one specimen in 1524 meters. North Indian Ocean, one deep-abyssal record.

Length.-277-394 mm.

\section{Mixonus pectoralis Goode and Bean}

Bathyonus pectoralis Goode and Bean, 1885, Proc. U. S. Nat. Mus., 8: 604 -Gulf of Mexico, ca. $28^{\circ} \mathrm{N} ., 87^{\circ} \mathrm{W}$.

Nematonus pectoralis Günther, 1887, Rep. Sci. Res. Voy. Challenger, Zool., 22: 114; Goode and Bean, 1895, Ocean. Ichth., p. 333, fig. 295; Jordan and Evermann, 1898, Bull. U. S. Nat. Mus., 47: 2518.

Mixonus pectoralis Norman, 1939, Sci. Rep. John Murray Exp. 1933-34, 7, (1), p. 88 .

Deep-abyssal record.-Western Atlantic, 2615 meters, the type.

Distribution.-Western Atlantic, one deep-abyssal record in the Gulf of Mexico and one young specimen off Dominica in 604 meters.

Length. $-70,215 \mathrm{~mm}$.

\section{Mastigopterus praetor Smith and Radcliffe}

Mastigopterus praetor Smith and Radcliffe, in Radcliffe, 1913, Proc. U. S. Nat. Mus., 44: 160, pl. 12, fig. 2-Patiente Strait, Dutch East Indies.

Mastigopterus praetor de Beaufort and Chapman, 1951, Fishes Indo-Austr. Arch., 9: 434, fig. 76.

Deep-abyssal record and distribution.-Western Pacific, ca. $0^{\circ} 28^{\prime}$ S., $127^{\circ}$ E., 2361 meters, one specimen.

Length. - $384 \mathrm{~mm}$.

\section{Grimaldichthys squamosus Roule}

Grimaldichthys squamosus Roule, 1916, Bull. Inst. Océanogr. Monaco, 320 : 19-Atlantic, ca. $36^{\circ}$ N., $22^{\circ} \mathrm{W}$.

Grimaldichthys Roule, 1915, C. R. Acad. Sci. Paris, 161 : 56.

Grimaldichthys squamosus Roule, 1919, Rés. Camp. Sci. Monaco, 52: 70, pl. 2, fig. 1; 1934, Poiss. Monde Viv. Eaux, 7 : 189; Fowler, 1936, Bull. Amer. Mus. Nat. Hist., 70: 1066, fig. 445; Belloc, 1949, Bull. Inst. Océanogr. Monaco, 958: 15 .

[Mastigopterus] squamosus Norman, 1939, Sci. Rep. John Murray Exp. 1933$34,7,(1)$, p. 88.

Deep-abyssal record and distribution.-Eastern Atlantic, 4621 meters, one specimen.

Length. $-286 \mathrm{~mm}$. 


\section{Grimaldichthys profundissimus Roule}

Grimaldichthys profundissimus Roule, 1913, Bull. Inst. Océanogr. Monaco, 261 : 3, fig.-Atlantic, ca. $12^{\circ}$ N., $33^{\circ}$ W., 6035 meters.

Grimaldichthys profundissimus Roule, 1916, Bull. Inst. Océanogr. Monaco, 320 : 19; 1919, Rés. Camp. Sci. Monaco, 52: 67, pl. 2, fig. 2; 1934, Poiss. Monde Viv. Eaux, $7: 187$, pl. 11, text fig.; Richard, 1934, Rés. Camp. Sci. Monaco, 89: 298; Fowler, 1936, Bull. Amer. Mus. Nat. Hist., 70: 1066; Belloc, 1949, Bull. Inst. Océanogr. Monaco, 958: 15, pl. 1; Nybelin, 1951, Rep. Swedish Deep-sea Exp. 1947-1948, Zool., 2, (1), pp. 15, 21; 1953, 14 Intern. Congr. Zool. Copenhagen, p. 1.

[Mastigopterus] profundissimus Norman, 1939, Sci. Rep. John Murray Exp. $1933-34,7$, (1), p. 88.

Deep-abyssal records and distribution.-Mid-Atlantic east of the mid-Atlantic ridge, 6035 meters, the type.

Mid-Atlantic east of the mid-Atlantic ridge, ca. $9^{\circ} \mathrm{N} ., 26^{\circ} \mathrm{W}$, 5600-5610 meters, one specimen.

Western Atlantic, ca. $24^{\circ} \mathrm{N} ., 63^{\circ} \mathrm{W}, 5850-5860$ meters, one specimen.

Known so far only from the abyssal plain.

Length.--?-220 mm.

Remarks.-A brief discussion of the haul made in 6035 meters may be found in Richard $(1902$, p. 85). For many years this was the deepest known record for any fish.

\section{Leucicorus lusciosus Garman}

Leucicorus lusciosus Garman, 1899, Mem. Mus. Comp. Zool., 24: 146, 361, pl. 38, figs. 1-37, pl. 74, fig. 1-Pacific off Mexico, ca. $14^{\circ} \mathrm{N} ., 98^{\circ} \mathrm{W}$.

Leucicorus lusciosus Lendenfeld, 1905, op. cit., 30: 198, pl. 3, figs. 10-13; Trojan, 1906, op. cit., p. 221, pls. 1, 2.

Deep-abyssal record and distribution.-Eastern Pacific, 3436 meters, one specimen.

Length. $-279 \mathrm{~mm}$.

\section{Enchelybrotula paucidens Smith and Radcliffe}

Enchelybrotula paucidens Smith and Radcliffe, in Radcliffe, 1913, Proc. U. S. Nat. Mus., 44: 154, pl. 11, fig. 1-Gulf of Tomini, Celebes.

Enchelybrotula paucidens de Beaufort and Chapman, 1951, Fishes Indo-Austr. Arch., 9: 437, fig. 78.

Deep-abyssal record and distribution.-Western Pacific, 1992 meters, one specimen.

Length. $-547 \mathrm{~mm}$. 


\section{Acanthonus armatus Günther}

Acanthonus armatus Günther, 1878, Ann. Mag. Nat. Hist., (5), 2: 22-type locality not stated, designated as Challenger Station 218 , ca. $1^{\circ} \mathrm{S} ., 144^{\circ} \mathrm{E}$., 1956 meters, the locality of the specimen figured by Günther (1887).

Acanthonus armatus Günther, 1887, Rep. Sci. Res. Voy. Challenger, Zool., 22: 117, pl. 24, fig. A; Goode and Bean, 1895, Ocean. Ichth., p. 336; Alcock and McGilchrist, 1905, Ill. Zool. Investigator, Fishes, pl. 38, figs. 1 and 1, b; Koefoed, 1927, Rep. Sci. Res. M. Sars No. Atl. Deep-sea Exp. 1910, 4, (1), p. 140; de Beaufort and Chapman, 1951, Fishes Indo-Austr. Arch., 9: 409 , fig. 64 .

Deep-abyssal records and distribution.-Eastern Atlantic, ca. $27^{\circ}$ N., $14^{\circ}$ W., 2603 meters, one specimen.

Western Pacific north of New Guinea, 1956 meters, the type.

Western Pacific off the Philippines, ca. $16^{\circ}$ N., $119^{\circ}$ E., 1920 meters, one specimen.

Probably a deep-abyssal species.

Length.-292-350 mm.

Remarks. - I have been unable to find any data on the specimen figured (pl. 38) in Illustrations of the Zoology of the Investigator, Fishes (1905). (See p. 92 for a second eastern Atlantic specimen.)

\section{Acanthonus spinifer Garman}

Acanthonus spinifer Garman, 1899, Mem. Mus. Comp. Zool., 24: 170, pl. F, fig. 3-Pacific off Panama, ca. $4^{\circ} \mathrm{N} ., 80^{\circ} \mathrm{W}$.

Deep-abyssal record and distribution.-Eastern Pacific, 3240 meters, one specimen.

Length.-Ca. $76 \mathrm{~mm}$.

\section{Tauredophidium hexti Alcock}

Tauredophidium Hextii Alcock, 1890, Ann. Mag. Nat. Hist., (6), 6: 213, pl. 8, fig. $1-$ Bay of Bengal, ca. $18^{\circ}$ N., $85^{\circ} \mathrm{E}$.

Tauredophidium Hextii Alcock, 1891, op. cit., (6), 7: 11; 1899, Descr. Cat. Indian Deep-sea Fishes, p. 97; 1902, Nat. Indian Seas, p. 236, fig. 34; Goode and Bean, 1895, Ocean. Ichth., p. 337, fig. 296, b; Alcock and Anderson, 1898, Ill. Zool. Investigator, Fishes, pl. 21, fig. 3; Misra, 1950,

Rec. Indian Mus., 45: 410; 1953, op. cit., 50: 392, fig. 11, $a$.

Deep-abyssal record and distribution.-North Indian Ocean, Bay of Bengal, 2395 meters, three specimens in one haul.

Length.--?-102 $\mathrm{mm}$.

\section{Typhlonus nasus Günther}

Typhlonus nasus Günther, 1878, Ann. Mag. Nat. Hist., (5), 2: 21-type locality not stated, designated as Challenger Station 181 , ca. $13^{\circ} \mathrm{S} ., 151^{\circ}$ E., 4461 meters, the locality of the specimen figured in Günther (1887). 
Typhlonus nasus Günther, 1887, Rep. Sci. Res. Voy. Challenger, Zool., 22: 119, pl. 25, fig. A; Goode and Bean, 1895, Ocean. Ichth., p. 340; de Beaufort and Chapman, 1951, Fishes Indo-Austr. Arch., 9: 408, fig. 63; Spärck, 1952, Danish Foreign Office Jour., 6: 6, fig.; Marshall, 1954, Aspects Deep Sea Biol., p. 249.

Deep-abyssal records and distribution.-Western Pacific northeast of Australia, 4461 meters, one specimen.

Western Pacific north of Celebes, ca. $2^{\circ}$ N., $124^{\circ}$ E., 3932 meters, one specimen.

Length.-?-254 mm.

Remarks.-The Galathea took five specimens in one haul near the Philippines, not far from the type locality (depth not stated; probably deep-abyssal).

\section{Cataetyx simus Garman}

Cataetyx simus Garman, 1899, Mem. Mus. Comp. Zool., 24: 168, 362, pl. E, fig. 2, pl. 39, figs. 3, 4, pl. 80, fig. 2-Pacific off Panama and between Ecuador and the Galapagos Islands (type locality not stated).

Deep-abyssal records and distribution.-Eastern Pacific, ca. $6^{\circ}$ N., $83^{\circ} \mathrm{W} ., 2690$ meters, (?) specimens.

Eastern Pacific, $0^{\circ} 36^{\prime}$ S., ca. $86^{\circ} \mathrm{W}$., 2417 meters, (?) specimens. Eastern Pacific, ca. $7^{\circ}$ N., $79^{\circ}$ W., 2322 meters, (?) specimens. Length.-?-483 $\mathrm{mm}$.

\section{Diplacanthopoma brunnea Smith and Radcliffe}

Diplacanthopoma (Sarcocara) brunnea Smith and Radcliffe, in Radcliffe, 1913, Proc. U. S. Nat. Mus., $44:$ 167, pl. 13, fig. 3-Palawan Passage, Philippine Islands, 685 meters.

Diplacanthopoma brunnea Cockerell, 1916, Ann. Mag. Nat. Hist., (8), 18: 320; Norman, 1939, Sci. Rep. John Murray Exp. 1933-34, 7, (1), p. 79.

Deep-abyssal record.—Arabian Sea, ca. $22^{\circ}$ N., 64 E., 1893 meters, one specimen.

Distribution.-Western Pacific, the type in 685 meters. North Indian Ocean, one deep-abyssal specimen.

Length. $-200-450 \mathrm{~mm}$.

\section{Aphyonus gelatinosus Günther}

Aphyonus gelatinosus Günther, 1878, Ann. Mag. Nat. Hist., (5), 2 : 22-Pacific south of New Guinea, ca. $12^{\circ} \mathrm{S} ., 145^{\circ} \mathrm{E}$.

Aphyonus gelatinosus Günther, 1887, Rep. Sci. Res. Voy. Challenger, Zool., 22: 120, pl. 26, fig. A; Goode and Bean, 1895, Ocean. Ichth., p. 342; Marshall, 1954, Aspects Deep Sea Biol., p. 249. 
Deep-abyssal record and distribution.-Western Pacific, 2560 meters, one specimen.

Length. $-140 \mathrm{~mm}$.

\section{Sciadonus pedicellaris Garman}

Sciadonus pedicellaris Garman, 1899, Mem. Mus. Comp. Zool., 24: 172, pl. F, fig. 4-Pacific off Panama, ca. $5^{\circ} \mathrm{N} ., 86^{\circ} \mathrm{W}$.

Deep-abyssal record and distribution.-Eastern Pacific, 1847 meters, (?) specimens.

Length. $-105 \mathrm{~mm}$.

\section{Barathronus affinis Brauer}

Barathronus affinis Brauer, 1906, Wiss. Ergebn. Deutschen Tiefsee Exp. Valdivia, 15, (1), p. 306-Indian Ocean, ca. $1^{\circ} \mathrm{S} ., 73^{\circ} \mathrm{E}$.

Barathronus affinis Brauer, 1908, op. cit., 15, (2), p. 162, pl. 35, figs. 11-13, text fig. 3 .

Deep-abyssal record and distribution.-North Indian Ocean, 2919 meters, one specimen.

Length. $-48 \mathrm{~mm}$.

\section{Barathronus parfaiti Vaillant}

Alexterion parfaiti Vaillant, 1888, Exp. Sci. Trav. Talis., Poiss., p. 283, pl. 25, fig. 2-Atlantic between France and the Azores, 5005 meters (see footnote, p. 110).

Alexterion parfaiti Goode and Bean, 1895, Ocean. Ichth., p. 343, fig. 300.

Barathronus parfaiti Roule, 1915, C. R. Acad. Sci. Paris, 161: 56; 1916, Bull. Inst. Océanogr. Monaco, 320 : 18; 1919, Rés. Camp. Sci. Monaco, 52: 73, pl. 2, fig. 4; 1934, Poiss. Monde Viv. Eaux, 7: 194, pl. 11, text fig.; Legendre, 1934, Ann. Inst. Océanogr. Paris, 14: 406, fig. 52; Fowler, 1936, Bull. Amer. Mus. Nat. Hist., 70: 1071.

Deep-abyssal records.-Eastern Atlantic, 5005 meters, the type.

Eastern Atlantic near the Azores, ca. $39^{\circ}$ N., $30^{\circ}$ W., 1846 meters, one specimen.

Distribution.-Eastern Atlantic, two deep-abyssal records and one young specimen from the stomach of a tuna caught in the Gulf of Gascony.

\section{Length. $-33-117 \mathrm{~mm}$.}

Remarks.-The specimen (33 mm.) taken from a tuna's stomach shows that the young of this species are probably pelagic, thus raising considerable doubt that the type, which was only $42 \mathrm{~mm}$. long, came from the bottom. However, the largest example was 
taken in a bottom haul with typically benthic fishes. Nybelin (1953) considered the species to be pelagic.

\section{Leucochlamys cryptophthalmus Zugmayer}

Leucochlamys cryptophthalmus Zugmayer, 1911, Bull. Inst. Océanogr. Monaco, 193: 11 -Atlantic, ca. $44^{\circ} \mathrm{N} ., 10^{\circ} \mathrm{W}$.

Leucochlamys cryptophthalmus Zugmayer, 1911, Rés. Camp. Sci. Monaco, 35 : 131, pl. 6, fig. 4; Roule, 1934, Poiss. Monde Viv. Eaux, 7 : 192-193, pl. 11, text fig.; Belloc, 1949, Bull. Inst. Océanogr. Monaco, 958: 15, pl. 3.

Deep-abyssal record and distribution.-Eastern Atlantic, 5000 meters, one specimen.

Length. $-85 \mathrm{~mm}$. (without caudal).

Remarks.-This specimen may have been caught in mid-water. The only other fish in the dredge was a Gonostoma.

\section{Family SCORPAENIDAE}

Apparently there are no scorpaenids known from deep-abyssal waters. A specimen of Pontinus kuhlii Bowdich was reported from a depth of 2330 meters by Vaillant $(1888$, p. 372$)$ but the species is not included in the list of deep-abyssal fishes because it is an archibenthic fish not otherwise known to reach below 1098 meters.

\section{Family COTTIDAE}

Cottids are principally north Pacific fishes but are also represented in the north Atlantic and Arctic oceans, as well as in fresh water in the northern hemisphere. One species is found in the southern hemisphere. Deep-sea species are chiefly archibenthic.

\section{Zesticelus profundorum Gilbert}

Acanthocottus profundorum Gilbert, 1895, Rep. U. S. Comm. Fish, 1893: 423, pl. 27 -Bering Sea, ca. $53^{\circ}$ N., $167^{\circ}$ W., 730 meters.

Zesticelus profundorum Jordan and Evermann, 1896, op. cit., 1895: 443; 1898, Bull. U. S. Nat. Mus., 47: 1990; 1900, op. cit., fig. 727; Jordan and Gilbert, 1899, Rep. Fur-seal Invest., 3: 467; Evermann and Goldsborough, 1907, Bull. U. S. Bur. Fish., 26: 315, fig. 75; Gilbert and Burke, 1912, op. cit., 30: 61; Gilbert, 1915, Proc. U. S. Nat. Mus., 48: 342; Townsend and Nichols, 1925, Bull. Amer. Mus. Nat. Hist., 52: 14; Andriashev, 1935, C. R. Acad. Sci. U.R.S.S., 4: 114; ${ }^{*} 1937$, Inst. Hydr. Leningrad, Expl. mers U.R.S.S., 25; 1955, U. S. Fish Wildlife Serv., Spec. Sci. Rep. Fish., 145 : 23, pl. 1, fig. 13; Bolin, 1944, Stanf. Ichth. Bull., 3, (1), p. 95, fig. 37. 
Deep-abyssal record.-Eastern Pacific, Monterey Bay, 1980 meters, one specimen.

Distribution.-Northern and eastern Pacific from Kamchatka and Bering Sea to ca. $33^{\circ} \mathrm{N}$., $120^{\circ} \mathrm{W}$., one deep-abyssal specimen and eight in 730-1178 meters. Probably accidental in deep-abyssal waters.

Length. -32 (standard) $-55 \mathrm{~mm}$.

\section{Family COTTUNCULIDAE}

Only one cottunculid species has been found in the deep-abyssal zone. Others are known from shallower parts of the deep sea in the Arctic, both sides of the north Atlantic, off South Africa, and in the tropical western Pacific.

\section{Cottunculoides spinosus Gilchrist}

Cottunculus spinosus Gilchrist, 1906, Mar. Invest. So. Afr., 4: 149, pl. 38off Cape Point, South Africa, 1463 meters.

Cottunculus spinosus Thompson, *1918, Mar. Biol. Rep. So. Afr., 4: 139; Gilchrist, 1922, Rep. Fish. Mar. Biol. Surv. So. Afr., 2, (3), p. 78.

Cottunculoides spinosus Barnard, 1927, Ann. Mag. Nat. Hist., (9), 20: 76; 1927, Ann. So. Afr. Mus., 21 : 924; Smith, 1949, Sea Fishes So. Afr., p. 376, fig. 1056.

Deep-abyssal record.-Off South Africa, ca. $33^{\circ}$ S., $17^{\circ}$ E., 2176 meters, one specimen.

Distribution.-Off Cape Point, South Africa, one deep-abyssal specimen and one in 1463 meters.

Length. $-45-78 \mathrm{~mm}$.

\section{Family LIPARIDAE}

A large family composed chiefly of benthic fishes, liparids are found principally but not exclusively in northern seas, ranging from tide pools to deep-abyssal waters. A few deep-sea species have taken to life in mid-water. Like the Cottidae, it is in cold and temperate north Pacific waters that the family has its largest population, although it is also well represented in the north Atlantic. A number of species are found in the Antarctic, several in temperate South Africa, seven in deep water in the tropical eastern Pacific, and one deep-sea species in the tropical Indian Ocean.

Thirteen species, belonging to four genera, are recorded from deep-abyssal waters but there is no positive indication that they 
are more than accidental there. One the other hand there is no proof that liparids known from only one or two specimens are not normal inhabitants of the deep-abyssal zone. Their absence in shallower hauls has some significance. All but three of the species under consideration here are from the northern and tropical eastern Pacific, and of these three, Paraliparis copei scarcely deserves a place on the list. Some may prove to be pelagic forms.

The only liparids known from the abyssal plain below 3660 meters are two unnamed forms, one of them from the north Pacific at a depth of 7200 meters, the other from the south Pacific in 6660 meters (see pp. 81-82).

Rhodichthys regina Collett. Table 24.

Rhodichthys regina Collett, *1878, Vidensk. Selsk. Forh., 1878, (14), p. $99-$ Arctic, ca. $72^{\circ} \mathrm{N} ., 5^{\circ}$ E., 2431 meters.

Rhodichthys regina Collett, 1880, Norske Nordhavs Exp. 1876-78, Zool., Fiske, p. 154, pl. 5, figs. 37-39; 1905, Rep. Norwegian Fish. Mar. Invest., 2, (3), p. 143, pl. 2, fig. 10; Günther, 1887, Rep. Sci. Res. Voy. Challenger, Zool., 22: 121; Lilljeborg, *1891, Sveriges Norges Fauna, Fisk., 2: 238; Goode and Bean, 1895, Ocean. Ichth., p. 342, fig. 303; Smitt, 1895, Skand. Fiskar, p. 596, fig. 142; Lütken, 1898, Danish Ingolf Exp., 2, (1), p. 31, pl. 3, fig. 4; Lönnberg, 1900, Rev. Intern. Pêche Pisc., 2, (4), p. 13; Ehrenbaum, 1902, Fauna Arct., 2: 118; Jensen, *1905, Medd. Komm. Havunders., Ser. Fisk., 1, (7); 1950, Vidensk. Medd. Dansk naturh. Foren., 112: 243, figs. 2, 3; Regan, 1912, Ann. Mag. Nat. Hist., (8), 10: 277; Murray and Hjort, 1912, Depths of Ocean, p. 436; Johnsen, 1921, Bergens Mus. Aarb., 1918-19, (6), pp. 24, 73, figs. 5-8; Essipov, 1937, Prob. Arctic, Arctic Inst. U.S.S.R., 4: 88, 94; 1939, Zool. Zhur., 18, (5), pp. 882, 887; Berg, 1940, Trav. Inst. Zool. Acad. Sci. U.R.S.S., 5: 331, 490; Andriashev, 1954, Fauna S.S.S.R., 53: 467, 542, figs. 272-274.

Deep-abyssal records.-Arctic, Kara Sea, ca. $82^{\circ}$ N., $87^{\circ}$ E., 2365 meters, one specimen.

Arctic, ca. $72^{\circ}$ N., $5^{\circ}$ E., 2341 meters, one specimen.

Arctic, $67^{\circ}-69^{\circ} \mathrm{N} ., 8^{\circ}-10^{\circ}$ and $61^{\circ}$ W., $1835-2000$ meters, at least five specimens, perhaps more, from five hauls.

Distribution.-Arctic only, in the west from Baffin Bay and Davis Strait (ca. $74^{\circ} \mathrm{N}$., $70^{\circ} \mathrm{W}$. to ca. $67^{\circ} \mathrm{N}$., $60^{\circ} \mathrm{W}$.) and in the east from the Laptev Sea (ca. $77^{\circ}$ N., $117^{\circ}$ E.) and north of Novaya Zemlya (ca. $82^{\circ} \mathrm{N}$., $87^{\circ} \mathrm{E}$.) to ca. $63^{\circ} \mathrm{N}$., $6^{\circ} \mathrm{W}$.; seven deep-abyssal records and at least twenty-seven specimens from between 1150 1320 and 1783 meters. Perhaps partially deep-abyssal in habitat but with its center of distribution somewhat higher.

Length. - 38-297 mm. 
Remarks.-Johnsen (1921, p. 73) wrote that the species might be pelagic, although it has been taken only in bottom-fishing appliances. It is apparently confined to waters of below-freezing temperatures.

\section{Careproctus bathycoetus Gilbert and Burke}

Careproctus bathycoetus Gilbert and Burke, 1912, Proc. U. S. Nat. Mus., 42: 368 , pl. 45 , fig. 10 -Okhotsk Sea, ca. $46^{\circ}$ N., $145^{\circ} \mathrm{E}$.

Careproctus bathycoetus Burke, 1930, Bull. U. S. Nat. Mus., 150: 129, fig. 50; Soldatov and Lindberg, *1930, Bull. Pac. Sci. Inst. Fish. Oceanogr., 5: 392; Schmidt, 1950, Akad. Sci. U.S.S.R. Trans. Pac. Comm., 6 : 208.

Deep-abyssal record and distribution.-Northwestern Pacific, 3291 meters, one specimen.

Length. $-181 \mathrm{~mm}$.

\section{Careproctus longifilis Garman}

Careproctus longifilis Garman, 1892, Mem. Mus. Comp. Zool., 14: 9-Pacific off Ecuador, ca. $2^{\circ} \mathrm{N} ., 83^{\circ} \mathrm{W}$.

Careproctus longifilis Garman, 1899, op. cit., 24: 114, pl. 27, fig. 1, pl. 28, fig. 1, pl. 29, fig. 5; Burke, 1930, Bull. U. S. Nat. Mus., 150: 130, fig. 1.

Deep-abyssal record and distribution.-Eastern Pacific, 3333 meters, one specimen.

Length. $-95 \mathrm{~mm}$.

\section{Careproctus ovigerum Gilbert}

Bathyphasma ovigerum Gilbert, 1896, Rep. U. S. Comm. Fish, 1893: 448Pacific off the Queen Charlotte Islands, ca. $52^{\circ} \mathrm{N} ., 132^{\circ} \mathrm{W}$.

Bathyphasma ovigerum Jordan and Evermann, 1898, Bull. U. S. Nat. Mus., 47: $2128 ; 1900$, op. cit., fig. 767.

Careproctus ovigerum Burke, 1930, op. cit., 150: 131, fig. 52; Clemens and Wilby, 1946, Bull. Fish. Res. Bd. Canada, 68: 302, fig. 224.

Deep-abyssal record and distribution.-Eastern Pacific, 2904 meters, one specimen.

Length. $-315 \mathrm{~mm}$.

Careproctus opisthotremus Gilbert and Burke

Careproctus opisthotremus Gilbert and Burke, 1912, Bull. U. S. Bur. Fish., 30 :

78 , fig. $23-$ Bering Sea, ca. $52^{\circ}$ N., $174^{\circ}$ E.

Careproctus opisthotremus Burke, 1930, Bull. U. S. Nat. Mus., 150 : 133, fig. 53.

Deep-abyssal record and distribution:-North Pacific, 1914 meters, one specimen.

Length. $-50 \mathrm{~mm}$. 


\section{Paraliparis fimbriatus Garman}

Paraliparis fimbriatus Garman, 1892, Mem. Mus. Comp. Zool., 14: 9Pacific off Colombia, ca. $4^{\circ} \mathrm{N} ., 80^{\circ} \mathrm{W}$.

Paraliparis fimbriatus Garman, 1899, op. cit., 24: 116, pl. D, fig. 3, pl. 29,

fig. 1; Burke, 1930, Bull. U. S. Nat. Mus., 150: 167, fig. 84.

Deep-abyssal record and distribution.-Eastern Pacific, 3240 meters, (?) specimens.

Length. - $102 \mathrm{~mm}$.

\section{Paraliparis ulochir Gilbert}

Paraliparis ulochir Gilbert, 1896, Rep. U. S. Comm. Fish, 1893: 441-Gulf of California, ca. $27^{\circ} \mathrm{N} ., 111^{\circ} \mathrm{W}$.

Paraliparis ulochir Jordan and Evermann, 1898, Bull. U. S. Nat. Mus., 47 : 2144; Gilbert, 1915, Proc. U. S. Nat. Mus., 48: 354; Burke, 1930, Bull.

U. S. Nat. Mus., 150 : 171, fig. 89; Böhlke, 1953, Stanf. Ichth. Bull., 5 : 138.

Deep-abyssal record.-Eastern Pacific, 1838 meters, the types (number of specimens not stated).

Distribution.-Eastern and northern Pacific, one deep-abyssal record in the Gulf of California, two specimens from Monterey Bay in $1383-1592$ meters and one from Bering Sea (ca. $54^{\circ}$ N., $166^{\circ}$ W.) in 743 meters.

Length.-?-85 mm.

\section{Paraliparis bathybii Collett}

Liparis (Paraliparis) bathybii Collett, *1879, Vidensk. Selsk. Forh., 1878: 32 Arctic, ca. $74^{\circ}$ N., $14^{\circ}$ E., 1203 meters.

Paraliparis bathybii (bathybius of authors) Collett, 1880, Norske Nordhavs Exp. 1876-78, Zool., Fiske, p. 52, pl. 2, fig. 14; 1905, Rep. Norwegian Fish. Mar. Invest., 2, (3), p. 104, pl. 2, fig. 9; Günther, 1887, Rep. Sci. Res. Voy. Challenger, Zool., 22: 68, pl. 12, fig. C; Garman, 1892, Mem. Mus. Comp. Zool., 14: 81; Goode and Bean, 1895, Ocean. Ichth., p. 279; Lütken, 1898, Danish Ingolf Exp., 2, (1), p. 17; Lönnberg, 1900, Rev. Intern. Pêche Pisc., 2, (4), p. 13; Ehrenbaum, 1902, Fauna Arct., 2: 101; Jensen, *1905, Medd. Komm. Havunders., Ser. Fisk., 1, (7); 1950, Vidensk. Medd. Dansk naturh. Foren., 112: 243, figs. 1, 3; Murray and Hjort, 1912, Depths of Ocean, pp. 126-127, 436-437, 641, 688, fig. 107; Johnsen, 1921, Bergens Mus. Aarb., 1918-19, (6), pp. 17, 70, pl., fig. 1, text figs. 2-4; Burke, 1930, Bull. U. S. Nat. Mus., 150 : 172; Andriashev, 1954, Fauna S.S.S.R., 53: 465,542 , figs. $271,272$.

Deep-abyssal records.-Arctic between Jan Mayen and Greenland, 2000 meters, one specimen.

Arctic, ca. $67^{\circ} \mathrm{N} ., 10^{\circ} \mathrm{W}$., 1847 meters, one specimen.

Arctic, ca. $69^{\circ} \mathrm{N} ., 8^{\circ} \mathrm{W} ., 1835$ meters, six specimens. 
Distribution.-Aretic only, in Davis Strait (ca. $67^{\circ} \mathrm{N}$., $60^{\circ} \mathrm{W}$.) and from ca. $74^{\circ} \mathrm{N}$., $14^{\circ} \mathrm{E}$. and $69^{\circ} \mathrm{N}$., $8^{\circ} \mathrm{W}$. to the Faroe Channel, three deep-abyssal records and forty-nine specimens in 670-1783 meters. Only two examples known from above 1200 meters. Probably reaching the deep-abyssal zone only occasionally.

Length.-179-253 mm.

Remarks. - There is some doubt concerning the habitat of the species, as it has been taken twice in mid-water. One of these pelagic specimens, a female containing large eggs, came aboard alive from a depth of only 670 meters (Johnsen, 1921, p. 70). On the other hand thirty-four specimens were taken in one trawl haul made at a depth of 1783 meters and there have been other catches of two to seven specimens each in bottom-fishing appliances. Johnsen (op. cit., p. 69) suggested that $P$. bathybii may be a pelagic species that seeks bottom for spawning purposes, or an entirely pelagic species that gathers in shoals at spawning time.

\section{Paraliparis latifrons Garman}

Paraliparis latifrons Garman, 1899, Mem. Mus. Comp. Zool., 24: 120, pl. 27, fig. 2, pl. 28, fig. 2-Pacific off Panama, ca. $6^{\circ} \mathrm{N}$., $80^{\circ} \mathrm{W}$.

Paraliparis latifrons Burke, 1930, Bull. U. S. Nat. Mus., 150 : 174, fig. 91.

Deep-abyssal record and distribution.-Eastern Pacific, 3279 meters, seven specimens in one haul.

Length.-?-145 $\mathrm{mm}$.

\section{Paraliparis holomelas Gilbert}

Paraliparis holomelas Gilbert, 1896, Rep. U. S. Comm. Fish, 1893: 441Bering Sea, ca. $54^{\circ}$ N., $166^{\circ}$ W., 743 meters.

Paraliparis holomelas Jordan and Evermann, 1898, Bull. U. S. Nat. Mus., 47 : 2140; Johnsen, 1921, Bergens Mus. Aarb., 1918-19, (6), pp. 20, 71, fig. 1; Burke, 1930, Bull. U. S. Nat. Mus., 150:175, fig. 92; Schmidt, 1950, Akad. Sci. U.S.S.R. Trans. Pac. Comm., 6: 222; Böhlke, 1953, Stanf. Ichth. Bull., 5: 138 .

Deep-abyssal records. - Northern Okhotsk Sea off Cape Elizabeth, 3350 meters, one specimen.

Bering Sea, ca. $56^{\circ}$ N., $172^{\circ}$ W., 2971 meters, one specimen.

Distribution.-North Pacific in the Okhotsk and Bering Seas, two deep-abyssal specimens and two in 743 and 128 meters.

Length. - 53-100 mm.

Remarks.-Specimens reported from Alaska by Evermann and Goldsborough (1907, p. 334) belong to P. deani Burke (1930, p. 168). 


\section{Paraliparis copei Goode and Bean}

Paraliparis copei Goode and Bean, 1895, Ocean. Ichth., p. 279, fig. 253-Atlantic off coast of United States, ca. $39^{\circ}$ N., $72^{\circ}$ W., 951 meters.

Paraliparis copei Jordan and Evermann, 1898, Bull. U. S. Nat. Mus., 47 : 2143; Roule, 1919, Rés. Camp. Sci. Monaco, 52: 62; Barnard, 1927, Ann. So. Afr. Mus., 21: 926; Burke, 1930, Bull. U. S. Nat. Mus., 150: 181, fig. 99; Smith, 1949, Sea Fishes So. Afr., p. 380, fig. 1073.

Deep-abyssal record.-Off Cape Point, South Africa, 1829 meters, one specimen.

Distribution.-Western Atlantic, ca. $39^{\circ}$ N., $70^{\circ}-74^{\circ}$ W., ten specimens, 548-1086 meters. Northwestern Atlantic, Davis Strait, ca. $63^{\circ} \mathrm{N}$., $55^{\circ} \mathrm{W}$., one specimen in 1200 meters. Eastern Atlantic, ca. $38^{\circ} \mathrm{N} ., 28^{\circ} \mathrm{W}$., three specimens in 1692 meters. South Africa off Cape Point, one deep-abyssal specimen and one in 1645 meters. Probably accidental in deep-abyssal waters.

Length. $-120-225 \mathrm{~mm}$.

\section{Paraliparis grandiceps Garman}

Paraliparis grandiceps Garman, 1899, Mem. Mus. Comp. Zool., 24: 117, pl. 29, fig. 4-Gulf of California, ca. $25^{\circ} \mathrm{N} ., 109^{\circ} \mathrm{W}$.

Paraliparis grandiceps Burke, 1930, Bull. U. S. Nat. Mus., 150: 183, fig. 101.

Deep-abyssal record and distribution.-Eastern Pacific, 2904 meters, one specimen.

Length. $-254 \mathrm{~mm}$.

Acantholiparis opercularis Gilbert and Burke

Acantholiparis opercularis Gilbert and Burke, 1912, Bull. U. S. Bur. Fish., 30 : 83, fig. 28 -Pacific off Kamchatka, ca. $52^{\circ}$ N., $158^{\circ}$ E., 1248 meters. Acantholiparis opercularis Burke, 1930, Bull. U. S. Nat. Mus., 150: 188, figs. 104, 105; Böhlke, 1953, Stanf. Ichth. Bull., 5: 135.

Deep-abyssal record.-North Pacific, ca. $53^{\circ}$ N., $159^{\circ}$ W., 3608 meters, one specimen.

Distribution.-North Pacific, one deep-abyssal record and two specimens off Kamchatka in 1248 meters.

Length.-?-76 mm.

\section{Family TRIACANTHODIDAE}

\section{Atrophacanthus danae Fraser-Brunner}

Atrophacanthus danae Fraser-Brunner, 1950, Dana Rep., 35: 3, figs. 1-5 -Celebes Sea. 
Distribution.-Western Pacific, Celebes Sea only, 156 specimens in 2500-3500 meters (4000-5000 meters of wire) and twenty-eight specimens in 300-2000 meters (600-3000 meters of wire), all over bottom depths ranging between 2160 and 4950 meters.

This is a bathypelagic species which, according to FraserBrunner $(1950$, p. 7$)$ lives and breeds over considerable depths and was absent from Dana hauls made over shallower water in the Celebes Sea.

Length.-2-44 mm. (standard).

Remarks.-Other members of the family live in shallower water and all may not be bathypelagic.

\section{Family CHAUNACIDAE}

Of the eight species of this small deep-sea family, only one has been reported from deep-abyssal waters. Barnard (1927, p. 1003) and other authors believe that the various species of the single genus Chaunax are synonymous. The fishes are found chiefly in tropical latitudes but also extend into temperate waters in the eastern Atlantic and off Australia.

\section{Chaunax roseus Barbour}

Chaunax roseus Barbour, 1941, Proc. New England Zool. Club, 19: 8, pl. 2 -off Cuba.

Deep-abyssal record and distribution.-Western Atlantic, 1956 meters, one specimen.

Length.-Ca. $219 \mathrm{~mm}$.

\section{Family OGOCEPHALIDAE}

Six ogocephalids, only one of them known from more than a few specimens, have been taken from the deep-abyssal zone. Other members of the family are known from both deep and shallow water in almost all tropical and temperate seas.

\section{Malthopsis spinosa Garman}

Malthopsis spinosa Garman, 1899, Mem. Mus. Comp. Zool., 24: 104, pl. 22 - Pacific off Panama, ca. $7^{\circ}$ N., $79^{\circ} \mathrm{W}$.

Deep-abyssal records and distribution.-Eastern Pacific, 2322, 1865 meters, an unstated number of specimens from two hauls.

Length.-Not stated. 


\section{Halieutopsis tumifrons Garman}

Halieutopsis tumifrons Garman, 1899, Mem. Mus. Comp. Zool., 24: 90, pl. 25 - Pacific near the Galapagos Islands.

Deep-abyssal records and distribution.-Eastern Pacific, ca. $2^{\circ} \mathrm{N}$, $92^{\circ}$ W., 2488 meters, (?) specimens.

Eastern Pacific, ca. $0^{\circ} 36^{\prime}$ S., $86^{\circ}$ W., 2417 meters, (?) specimens. Length.-Not stated.

\section{Dibranchus nasutus Alcock}

Dibranchus nasutus Alcock, 1891, Ann. Mag. Nat. Hist., (6), 8: 24, pl. 7, fig. 1-Bay of Bengal, ca. $11^{\circ}$ N., $92^{\circ}$ E., 343-403 meters.

Dibranchus nasutus Alcock and Anderson, 1898, Ill. Zool. Investigator, Fishes, pl. 20, fig. 2; Alcock, 1899, Descr. Cat. Indian Deep-sea Fishes, p. 63; 1902, Nat. Indian Seas, p. 247, fig. 46; Brauer, 1906, Wiss. Ergebn. Deutschen Tiefsee Exp. Valdivia, 15, (1), p. 328; Radcliffe, 1912, Proc. U. S. Nat. Mus., 42: 211, pl. 22, fig. 3, pl. 23, fig. 3; Weber, 1913, Monogr. Siboga Exp., 57: 568, pl. 9, figs. 1, 2; Norman, 1939, Sci. Rep. John Murray Exp. 1933-34, 7, (1), p. 113.

Deep-abyssal record.-Western Pacific, Banda Sea, 1886 meters, one specimen.

Distribution.-Western Pacific in Macassar Strait, Banda Sea and Timor Sea, one deep-abyssal record and three specimens in 724-1158 meters. North Indian Ocean from ca. $1^{\circ}$ S., $41^{\circ} \mathrm{E}$. and the Gulf of Aden to the Andaman Sea, at least five specimens, three records in 343-743 meters, one in 1270 meters and one in 1668 meters. Probably accidental in deep-abyssal waters.

Length. - $33-140 \mathrm{~mm}$.

\section{Dibranchus nudiventer Lloyd}

Dibranchus nudiventer Lloyd, 1909, Mem. Indian Mus., 2: 168, pl. 45, fig. 2 -Bay of Bengal off Arakan, 2012 meters.

Dibranchus nudiventer Norman, 1939, Sci. Rep. John Murray Exp. 1933-34, 7, (1), p. 113.

Deep-abyssal record.-North Indian Ocean, Bay of Bengal, 2012 meters, one specimen.

Distribution.-North Indian Ocean, one deep-abyssal record and one specimen near Zanzibar in 1789 meters.

Length. $-65-75 \mathrm{~mm}$.

\section{Dibranchus hystrix Garman}

Dibranchus hystrix Garman, 1899, Mem. Mus. Comp. Zool., 24: 92, pl. 23 -Pacific off Colombia and Panama (type locality not stated). 
Dibranchus hystrix Townsend and Nichols, 1925, Bull. Amer. Mus. Nat. Hist., 52: 18.

Deep-abyssal records.--Eastern Pacific, ca. $7^{\circ}$ N., $79^{\circ}$ W., 2322 meters, (?) specimens.

Eastern Pacific, ca. $2^{\circ}$ N., 89 W., 2196 meters, (?) specimens.

Eastern Pacific, ca. $5^{\circ} \mathrm{N}$., $85^{\circ} \mathrm{W}$., 2150 meters, (?) specimens.

Distribution.-Eastern Pacific, three deep-abyssal records, $2^{\circ}-7^{\circ}$ N., $79^{\circ}-89^{\circ} \mathrm{W}$.; and one specimen off Mexico, ca. $22^{\circ} \mathrm{N} ., 109^{\circ} \mathrm{W}$, 1153 meters.

Length.-Not stated.

Remarks. - The eastern Atlantic specimen reported by Murray and Hjort (1912, p. 411) was later identified as D. atlanticus by Koefoed (1927, p. 141).

\section{Dibranchus obscurus Brauer}

Dibranchus olscurus Brauer, 1906, Wiss. Ergebn. Deutschen Tiefsee Exp. Valdivia, 15, (1), p. 330-Gulf of Aden, 1840 meters.

Dibranchus obscurus Norman, 1939, Sci. Rep. John Murray Exp. 1933-34, 7, (1), p. 113, fig. 39.

Deep-abyssal record.-North Indian Ocean, Gulf of Aden, 1840 meters, one specimen.

Distribution.-North Indian Ocean in the Gulf of Aden and near Zanzibar, one deep-abyssal specimen and three in 1022-1789 meters.

Length. $-42-210 \mathrm{~mm}$.

\section{GERATIOIDEA}

Recent studies by Bertelsen (1951) have shown that the distribution of adult ceratioid fishes is nearly cosmopolitan, including temperate and cold waters in the north Atlantic, north Pacific, and southern oceans, but that larval stages have been found only in the warmer areas, between about $40^{\circ} \mathrm{N}$. and $35^{\circ} \mathrm{S}$.

All members of the order, which contains ten families, are apparently bathypelagic, the metamorphosing and adult specimens being found principally at or below a depth of about 2000 meters, according to Bertelsen. Larvae occur in much shallower water, above 200 meters, and adolescent females in 1500-2000 meters. Few species are represented in collections in enough quantity to permit an estimate of their maximum occurrence. Bertelsen found that Cryptopsaras couesi Gill (family Ceratiidae) apparently in- 
habits lesser depths than most species, while Melanocetus murrayi Günther (family Melanocetidae) lives somewhat deeper. Edriolychnus schmidti Regan (family Linophrynidae) probably inhabits deep-abyssal waters.

In view of the complexities of the vertical distribution of this group of fishes, metamorphosing, adolescent and adult specimens of all known species are listed. It is quite possible that some live in deep-abyssal waters as adolescents and adults. The presence well above the 2000-meter line of mature females of various species suggests that occurrence below that depth may in some cases be confined to males and unmated adult females (see Linophryne arborifera, p. 273).

Parasitic males have been discovered in four families: Caulophrynidae, Ceratiidae, Neoceratiidae and Linophrynidae. Judging from evidence that free-living males of the Himantolophidae and Melanocetidae grow and feed after metamorphosis and that those of the families Oneirodidae and Gigantactinidae have large testicles, Bertelsen has concluded that males of these four families probably never become parasitic, only attaching temporarily by means of their rostral denticles. Too little is known of males of the families Diceratiidae and Centrophrynidae to speculate upon their life histories.

For Dana records the depth of capture in relation to meters of wire has been taken from Bertin (1934, p. 29), as follows:

Meters of Wire
$1000-2000$
$3000-4000$
$5000-6000$

\section{Family CAULOPHRYNIDAE}

Bertelsen (1951) limited the family to one species with three subspecies, adult and adolescent specimens of which are listed below. Larvae have been taken in the Indian Ocean, Tasman Sea, western Pacific and eastern Atlantic. Nine adult or adolescent specimens have been recorded, five of them from deep-abyssal waters. Males are known to be parasitic.

\section{Caulophryne jordani jordani Goode and Bean}

Caulophryne jordani Goode and Bean, 1895, Ocean. Ichth., p. 496, fig. 409 -Atlantic off coast of United States, ca. $39^{\circ}$ N., $71^{\circ}$ W., 2334 meters. 
Caulophryne jordani Jordan and Evermann, 1898, Bull. U. S. Nat. Mus., 47 : 2735; 1900, op. cit., fig. 957; Gill, 1908, Ann. Rep. Smithsonian Inst., p. 585, fig. 24; Regan, 1912, Ann. Mag. Nat. Hist., (8), 9: 288; 1926, Oceanogr. Rep. Danish Dana Exp. 1920-22, 2: 22 (part), fig. 16; Beebe, 1926, Arcturus Adv., p. 422; 1929, Zoologica, 12:19; Regan and Trewavas, 1932, Dana Rep., 2: 101, fig. 159; Bertelsen, 1951, op. cit., 39: 33, fig. 8. Caulophryne Beebe and Rose, 1926, Bull. N. Y. Zool. Soc., 29: 53, fig.

Deep-abyssal record.-Western Atlantic, 2334 meters, one specimen.

Distribution.-Western Atlantic off New York, off New Jersey, and near Bermuda, one deep-abyssal record and two specimens in 1098 and 549-914 meters.

Length.-?-40 $\mathrm{mm}$.

\section{Caulophryne jordani pelagica Brauer}

Melanocetus pelagicus Brauer, 1902, Zool. Anz., 25: 295-Indian Ocean west of Chagos Archipelago, ca. $2^{\circ} \mathrm{S} ., 65^{\circ}$ E., 2500 meters.

Melanocetus pelagicus Brauer, 1906, Wiss. Ergebn. Deutschen Tiefsee Exp. Valdivia, 15, (1), p. 321 , pl. 15, fig. 5.

Caulophryne pelagicus Regan, 1912, Ann. Mag. Nat. Hist., (8), 9: 288.

Caulophryne jordani Regan, 1926, Oceanogr. Rep. Danish Dana Exp. 1920-22, 2: 22 (part).

Caulophryne pelagica Regan and Trewavas, 1932, Dana Rep., 2: 102, fig. 162.

Caulophryne ramulosa Regan and Trewavas, 1932, op. cit., p. 101, pl. 7, text fig. 160 .

Caulophryne acinosa Regan and Trewavas, 1932, op. cit., p. 101, pl. 8, fig. 1, text fig. 61 .

Caulophryne jordani pelagica Bertelsen, 1951, op. cit., 39: 33, 37, figs. 9, 10, $11, c$.

Deep-abyssal records and distribution.-North Indian Ocean, ca. $1^{\circ}-2^{\circ}$ S., $65^{\circ}$ E., 2500,2200 meters, two specimens.

Western Pacific, ca. $15^{\circ}$ N., $115^{\circ}$ E. (South China Sea), 2000 meters, one metamorphosing male, the only known free-living male specimen of the family.

Eastern Pacific, Gulf of Panama, 2000 meters, one specimen.

Length.-11-90 mm.

\section{Caulophryne jordani polynema Regan}

Caulophryne polynema Regan, 1930, Jour. Linn. Soc. London, 37: 191, figs. 1-3 - Madeira.

Caulophryne polynema Parr, 1930, Copeia, p. 131, fig. 4; Regan and Trewavas, 1932, Dana Rep., 2: 14, 101, fig. 2; Fowler, 1936, Bull. Amer. Mus. Nat. Hist., 70 : 1347. 
Ceratocaulophryne regani Roule and Angel, 1932, Bull. Mus. Hist. Nat. Paris, 4: 500; 1933, Rés. Camp. Sci. Monaco, 86: 55, pl. 3, figs. 26, 26, $a$; Belloc, 1949, Bull. Inst. Océanogr. Monaco, 958: 17.

Caulophryne regani Fowler, 1936, Bull. Amer. Mus. Nat. Hist., 70: 1347.

Caulophryne jordani polynema Bertelsen, 1951, Dana Rep., 39: 33.

Deep-abyssal record.-Mid-Atlantic, ca. $38^{\circ}$ N., $34^{\circ}$ W., 3000 meters, one specimen.

Distribution.-Atlantic, one deep-abyssal record and one female specimen with a parasitic male taken on a line in deep water off Madeira (eastern Atlantic).

Length. $-59-210 \mathrm{~mm}$.

\section{Family MELANOCETIDAE}

According to the latest revision the family contains only the genus Melanocetus Günther, with nine species, although Bertelsen (1951) explained that the separation of the species is still uncertain.

Melanocetus murrayi Günther. Table 25.

Melanocetus murrayi Günther, 1887, Rep. Sci. Res. Voy. Challenger, Zool., 22: 57, pl. 11, fig. A-mid-Atlantic, ca. $1^{\circ}$ N., $24^{\circ}$ W., 3384 meters.

Melanocetus bispinosus Günther, 1880, Intr. Study Fishes, p. 473 (name only). Melanocetus (Liocetus) murrayi Günther, 1887, Rep. Sci. Res. Voy. Challenger, Zool., 22: 56.

Liocetus murrayi Goode and Bean, 1895, Ocean. Ichth., p. 495, fig. 407; Gill, 1908, Ann. Rep. Smithsonian Inst., p. 583, fig. 22.

Melanocetus vorax Brauer, 1902, Zool. Anz., 25: 294; 1906, Wiss. Ergebn. Deutschen Tiefsee Exp. Valdivia, 15, (1), p. 320, pl. 15, fig. 4; Fowler, 1936, Bull. Amer. Mus. Nat. Hist., 70: 1144.

Melanocetus johnsoni Brauer, 1906, Wiss. Ergebn. Deutschen Tiefsee Exp. Valdivia, 15, (1), p. 319, pl. 15, fig. 3; Regan, 1926, Oceanogr. Rep. Danish Dana Exp. 1920-22, 2: 33 (part); Murray and Hjort, 1912, Depths of Ocean, pp. 609, 614, 618, fig. 469.

Melanocetus krechi Murray and Hjort, 1912, Depths of Ocean, pp. 614 (part), 618.

Melanocetus murrayi Regan, 1926, Oceanogr. Rep. Danish Dana Exp. 1920-22, 2: 32; Parr, 1927, Bull. Bingham Oceanogr. Coll., 3, (1), p. 27; 1934, op. cit., 4, (6), p. 7; Regan and Trewavas, 1932, Dana Rep., 2: 49, fig. 71; Beebe, 1932, Zoologica, 13: 99, figs. 29, 30; Koefoed, 1944, Rep. Sci. Res. M. Sars No. Atl. Deep-sea Exp. 1910, 4, (2), p. 3; Bertelsen, 1951, Dana Rep., 39: 44, fig. 16; Grey, 1955, Fieldiana, Zool., $37: 299$.

Melanocetus niger Parr, 1927, Bull. Bingham Oceanogr. Coll., 3, (1), p. 29; Beebe, 1929, Zoologica, 12: 18; ? Gregory, 1933, Trans. Amer. Phil. Soc., 23 : 400 , fig. 272. 


\section{SYNONYMY OF MALES}

Rhynchoceratias acanthirostris Parr, 1927, Bull. Bingham Oceanogr. Coll., 3, (1), p. 31, fig. 11.

Rhynchoceratias latirhinus Parr, 1927, op. cit., p. 32, fig. 12.

Rhynchoceratias longipinnis Parr, 1930, Occ. Pap. Bingham Oceanogr. Coll., 3: 7, figs. 1-7.

Xenoceratias acanthirostris Regan and Trewavas, 1932, Dana Rep., 2 : 55.

Xenoceratias longipinnis Regan and Trewavas, 1932, op. cit., p. 56.

Xenoceratias latirhinus Regan and Trewavas, 1932, op. cit., p. 57.

? Xenoceratias regani Koefoed, 1944, Rep. Sci. Res. M. Sars No. Atl. Deep-sea Exp. 1910, 4, (2), p. 4, pl. 1, fig. 6 .

Distribution.-Eastern and mid-Atlantic from ca. $34^{\circ}$ N., $33^{\circ} \mathrm{W}$. to the Gulf of Guinea. Western Atlantic off New York to Bermuda, in the Gulf of Mexico and in the Caribbean Sea. Pacific in ca. $4^{\circ} \mathrm{S}$., $116^{\circ}$ W.; in the Banda Sea; and off Sydney, Australia, ca. $33^{\circ} \mathrm{S}$., $154^{\circ} \mathrm{E}$. More than fifty specimens known, about half of them deep-abyssal. Extreme depth range 1050-4480 meters.

Larvae have been found on both sides of the north Atlantic, in the south Atlantic, the south Pacific and the western Pacific.

Length. - $15-115 \mathrm{~mm}$. (without caudal).

\section{Melanocetus megalodontis Beebe and Crane}

Melanocetus megalodontis Beebe and Crane, 1947, Zoologica, 31 : 152, fig. 1Pacific, ca. $20^{\circ} \mathrm{N}$., $115^{\circ} \mathrm{W}$.

Melanocetus megalodontis Bertelsen, 1951, Dana Rep., 39: 48.

Distribution.-Eastern Pacific, 914 meters, one specimen.

Length. $-38.5 \mathrm{~mm}$.

Melanocetus johnsoni Günther. Table 26.

Melanocetus johnsoni Günther, 1864, Proc. Zool. Soc. London, p. 301, pl. 25 - Madeira.

Melanocetus johnsoni Lütken, *1871, Dansk Vidensk. Selsk. Skr., (5), 11, (5), pp. 64, 74; Günther, 1887, Rep. Sci. Res. Voy. Challenger, Zool., 22: 56; Vaillant, 1888, Exp. Sci. Trav. Talis., Poiss., p. 346; Goode and Bean, 1895, Ocean. Ichth., p.494, fig.406; Gill, 1908, Ann. Rep. Smithsonian Inst., p.582, fig. 20; Regan, 1913, Proc. Zool. Soc. London, p. 1096; 1926, Oceanogr. Rep. Danish Dana Exp. 1920-22, 2: 33; Parr, 1927, Bull. Bingham Oceanogr. Coll., 3, (1), p. 29; Norman, 1930, Disc. Rep., 2: 354; 1939, Sci. Rep. John Murray Exp. 1933-34, 7, (1), p. 114; Regan and Trewavas, 1932, Dana Rep., 2: 12, 49, 50, figs. 72, 73; Fowler, 1936, Bull. Amer. Mus. Nat. Hist., 70: 1143, fig. 482; Koefoed, 1944, Rep. Sci. Res. M. Sars No. Atl. Deep-sea Exp. 1910, 4, (2), p. 3; Beebe and Crane, 1947, 
Zoologica, 31 : 152; Bertelsen, 1951, Dana Rep., 39: 39, 48, figs. 13, 15, 17-19.

?Melanocetus krechi Brauer, 1902, Zool. Anz., 25: 293; 1906, Wiss. Ergebn.

Deutschen Tiefsee Exp. Valdivia, 15, (1), p. 319, pl. 15, figs. 1, 2; Gill, 1908, Ann. Rep. Smithsonian Inst., p. 583, fig. 21; Murray and Hjort, 1912, Depths of Ocean, pp. 87, 614 (part); Borodin, 1931, Bull. Mus. Comp. Zool., 72: 84; Regan and Trewavas, 1932, Dana Rep., 2: 52, fig. 74.

?Melanocetus rotundatus Gilchrist, 1903, Mar. Invest. So. Afr., 2: 206, pl. 15; Gilchrist and Thompson, *1917, Ann. Durban Mus., 1: 417; Thompson, *1918, Mar. Biol. Rep. So. Afr., 4 : 155; Barnard, 1927, Ann. So. Afr. Mus., 21 : 1007; Von Bonde, 1928, Rep. Fish. Mar. Biol. Surv. So. Afr., 5: 49; Smith, 1949, Sea Fishes So. Afr., p. 429, fig. 1232.

Melanocetus sp. Roule and Angel, 1930, Rés. Camp. Sci. Monaco, 79: 121, pl. 6, fig. 159.

Melanocetus cirrifer Regan and Trewavas, 1932, Dana Rep., 2: 52 (part).

\section{SYNONYMY OF MALES}

Centrocetus spinulosus Regan and Trewavas, 1932, Dana Rep., 2: 53, fig. 79. Xenoceratias micracanthus Regan and Trewavas, 1932, op. cit., p. 55, fig. 81. Xenoceratias heterorhynchus Regan and Trewavas, 1932, op. cit., p. 56, fig. 82. Xenoceratias laevis Regan and Trewavas, 1932, op. cit., p. 56, fig. 83.

Xenoceratias brevirostris Regan and Trewavas, 1932, op. cit., p. 57, fig. 84.

? Xenoceratias braueri Koefoed, 1944, Rep. Sci. Res. M. Sars No. Atl. Deep-sea Exp. 1910, 4, (2), p. 6, fig. 2.

Distribution.-Atlantic from ca. $41^{\circ}$ to $8^{\circ} \mathrm{N}$. and, in the east, south to ca. $35^{\circ} \mathrm{S}$., $18^{\circ} \mathrm{E}$. North Indian Ocean from off the CocosKeeling Islands and the Arabian Sea to off Durban, ca. $31^{\circ} \mathrm{S} ., 30^{\circ} \mathrm{E}$. Western Pacific in the South China Sea and Celebes Sea. South Pacific off New Caledonia, between New Zealand and the Kermadec Islands, and in ca. $4^{\circ} \mathrm{S}$., $116^{\circ} \mathrm{W}$. Gulf of Panama. Extreme depth range 0-4789 meters.

Larvae have been taken in the western, eastern and southeastern Atlantic, the Indian Ocean off South Africa, the north Indian Ocean and the western and southwestern Pacific.

About four hundred specimens are known in all, only eighty or so being adults, adolescents or metamorphosing examples. About half of these older specimens were caught at or below 2000 meters.

Length.-7-114 mm.

Remarks.-Four specimens found floating at the surface and three whose depth of capture is unknown have been omitted from Table 26. 


\section{Melanocetus niger Regan}

Melanocetus niger Regan, 1925, Ann. Mag. Nat. Hist., (9), 15: 565-Gulf of Panama, 2200 meters.

Melanocetus niger Regan, 1926, Oceanogr. Rep. Danish Dana Exp. 1920-22, 2: 33, pl. 8, fig. 1; Regan and Trewavas, 1932, Dana Rep., 2: 53, fig. 76, b; Beebe and Crane, 1947, Zoologica, 31: 153; Bertelsen, 1951, Dana Rep., 39: 53.

Deep-abyssal records.-Gulf of Panama, 2000-2300 meters, eight specimens from five hauls.

Distribution.-Eastern Pacific in the Gulf of Panama and south of Cocos Island, fifteen specimens, eight of them deep-abyssal, seven in $500-1700$ meters.

Length. $-18-113 \mathrm{~mm}$.

Remarks.-Males and larvae unknown.

\section{Melanocetus ferox Regan}

Melanocetus ferox Regan, 1926, Oceanogr. Rep. Danish Dana Exp. 1920-22, 2: 33, pl. 9, fig. 1-Gulf of Panama, 2000 meters.

Melanocetus ferox Regan and Trewavas, 1932, Dana Rep., 2: 52, fig. 75; Beebe and Crane, 1947, Zoologica, 31: 152; Bertelsen, 1951, Dana Rep., 39: 53.

Deep-abyssal records. - Gulf of Panama, 2500, 2000 meters, three specimens from three hauls.

Distribution.-Eastern Pacific off the Galapagos Islands, off Costa Rica, and in the Gulf of Panama. Five specimens known, three deep-abyssal, one in 914 meters and one in 549 meters.

Length. $-23.5-110 \mathrm{~mm}$.

Remarks.-The species is probably a variation of $M$. niger (Bertelsen, 1951, p. 53). Males and larvae unknown.

\section{Melanocetus cirrifer Regan and Trewavas}

Melanocetus cirrifer Regan and Trewavas, 1932, Dana Rep., 2: 52, pl. 2, fig. 1, text figs. 76, $a, 77$--Banda Sea.

Melanocetus cirrifer Bertelsen, 1951, op. cit., 39: 53.

Deep-abyssal records and distribution.-Western Pacific, Banda Sea, 2500 meters, the type.

Western Pacific, Celebes Sea, 2000 meters, one specimen.

Length.-24-39 mm.

Remarks.-Bertelsen (1951, p. 53) doubted the validity of this species. Males and larvae unknown. 
Melanocetus longirostris Regan and Trewavas

Xenoceratias longirostris Regan and Trewavas, 1932, Dana Rep., 2: 54, fig. 80 —north of New Guinea, ca. $3^{\circ} \mathrm{N}$., $137^{\circ} \mathrm{E}$.

Melanocetus longirostris Bertelsen, 1951, op. cit., 39: 54.

Deep-abyssal record and distribution.-Western Pacific, 2000 meters, the type, a male.

Length. $-29 \mathrm{~mm}$.

Remarks.-The only known specimen is probably the male of M. megalodontis, niger, ferox, or cirrifer (Bertelsen, 1951, p. 54).

\section{Melanocetus nudus Beebe and Crane}

Xenoceratias nudus Beebe and Crane, 1947, Zoologica, 31 : 155, fig. 2-Pacific, ca. $9^{\circ}$ N., $85^{\circ}$ W., 914 meters.

Melanocetus nudus Bertelsen, 1951, Dana Rep., 39: 54, fig. 20.

Deep-abyssal record.-Gulf of Panama, 3000 meters, one male specimen.

Distribution.-Eastern Pacific off Costa Rica and in the Gulf of Panama, one deep-abyssal specimen and one from 914 meters. Length.-29-31 mm.

Remarks.-Females and larvae unknown. This species is probably the male of $M$. megalodontis, niger, ferox or cirrifer and perhaps is not distinct from $M$. longirostris (Bertelsen, 1951, p. 54).

\section{Melanocetus polyactis Regan}

Melanocetus polyactis Regan, 1925, Ann. Mag. Nat. Hist., (9), 15: 565-Gulf of Panama, 2200 meters.

Melanocetus polyactis Regan, 1926, Oceanogr. Rep. Danish Dana Exp. 192022, 2: 34, pl. 8, fig. 2; Regan and Trewavas, 1932, Dana Rep., 2: 53, fig. 78; Bertelsen, 1951, op. cit., 39: 40, 54, figs. 14, 21.

\section{SYNONYMY OF MALES}

Rhynchoceratias rostratus Regan, 1926, Oceanogr. Rep. Danish Dana Exp. 1920-22, 2: 44 (part).

Rhynchoceratias leucorhinus Regan, 1926, op. cit., p. 44 (part).

Deep-abyssal records.-Gulf of Panama, 2200 meters, three specimens.

Gulf of Panama, 2000 meters, one specimen.

Distribution.-Eastern Pacific, only in the Gulf of Panama and in ca. $2^{\circ} \mathrm{N} ., 87^{\circ} \mathrm{W}$. Five specimens known, four deep-abyssal, one in 1500 meters. Larvae, six specimens, from ca. $2^{\circ} \mathrm{N} ., 87^{\circ} \mathrm{W}$.

Length. $-22-90 \mathrm{~mm}$. 


\section{Melanocetus tumidus Parr}

Melanocetus tumidus Parr, 1927, Bull. Bingham Oceanogr. Coll., 3, (1), p. 28, fig. 10-Atlantic off Bermuda.

Melanocetus tumidus Regan and Trewavas, 1932, Dana Rep., 2: 27.

Distribution.-Western Atlantic, only one specimen, a young female, 1409 meters.

Length. $-21 \mathrm{~mm}$.

Remarks.-Probably a young female $M$. murrayi, although not mentioned by Bertelsen (1951).

\section{Family HIMANTOLOPHIDAE}

Bertelsen (1951) considered valid three species of the single genus Himantolophus and retained a fourth until further material proves to which female species the two male specimens should be assigned. H. groenlandicus is one of the larger ceratioids, reaching a length of nearly two feet. The family is included here for the sake of completeness. There is no evidence that any of the species inhabits deep-abyssal waters. Males are probably free-living throughout life.

\section{Himantolophus groenlandicus Reinhardt}

Himantolophus groenlandicus Reinhardt, ${ }^{*} 1837$, Dansk Vidensk. Selsk. Afhandl., (4), $7: 116$, pl. 4-off Greenland.

Himantolophus groenlandicus Reinhardt, ${ }^{*} 1857$, Grønl. geogr. statisk. besk. H. Rink, 2: 23; Lütken, 1878, Dansk Vidensk. Selsk. Skr., (5), 11, (5), pl. 2, fig. 5; Günther, 1887, Rep. Sci. Res. Voy. Challenger, Zool., 22: 51; Girard, *1893, Bull. Soc. Geogr. Lisboa, 11 : 603, pl. 1; Goode and Bean, 1895, Ocean. Ichth., p. 493, fig. 405; Jordan and Evermann, 1898, Bull. U. S. Nat. Mus., 47: 2733; Ehrenbaum, 1902, Fauna Arct., 2: 76; Regan, 1926, Oceanogr. Rep. Danish Dana Exp. 1920-22, 2: 40, pl. 12, fig. 1, text fig. 23; Regan and Trewavas, 1932, Dana Rep., 2: 13, 59, pl. 1, fig. 1, text fig. 88; Nobre, 1935, Fauna Mar. Portugal, 1: 233, pl. 32, fig. 105, pl. 32, a, fig. 105, $a$, $b$; Barbour, 1942, Proc. New England Zool. Club, 21: 82, pl. 15; Coelho, 1942, Trav. Stat. Biol. Marit. Lisbonne, 46: 90; Bertelsen, 1951, Dana Rep., 39: 60, figs. 23-25, 26, $a-e$.

Himantolophus reinhardtii Lütken, 1878, Dansk Vidensk. Selsk. Skr., (5), 11, (5), p. 320 , pls. 1 , 2, figs. $1-4 ; 1887$, op. cit., (6), 4, (5), p. 325 , pl., text figs.; 1895, Vidensk. Medd. Dansk naturh. Foren., 1894: 78; Günther, 1887, Rep. Sci. Res. Voy. Challenger, Zool., 22: 51; Ehrenbaum, 1902, Fauna Arct., 2: 76 ; ${ }^{*} 1936$, Naturg. wirtsch. Bedeut. Seefische Nordeur., p. 163; Gill, 1908, Ann. Rep. Smithsonian Inst., p. 581, figs. 11, 18; Holt and Byrne, *1909, Mem. Challenger Soc., 1 : 195, pls. 1, 2; Osório, 1909, Mem. Mus. Bocage, Lisboa, 1, (1), p. 18, pl. 1, figs. 5, 6; Seabra, ${ }^{*} 1911$, Bull. Soc. 
Port. Sci. Nat., 5, (3), p. 163; Williamson, 1911, Ann. Rep. Fish. Bd. Scotland, 1909, (3), p. 51, pl. 3; Pappenheim, 1914, Deutsche SüdpolarExp. 1901-1903, 15, Zool., 7 : 198; Saemundsson, 1922, Vidensk. Medd. Dansk naturh. Foren., 74: 183; 1927, op. cit., 84 : 168; 1949, Zool. Iceland, 4, (72), p. 46 (further references, Iceland); Barnard, 1927, Ann. So. Afr. Mus., 21 : 1006, pl. 37, fig. 2; Fage, 1929, Faune Ichth. Atl. Nord, 2, figs.; Bigelow and Barbour, 1944, Proc. New England Zool. Club, 23: 14; Smith, 1949, Sea Fishes So. Afr., p. 429, fig. 1230; Rae, 1951, Ann. Biol. Cons. Perm. Intern. Explor. Mer, $7: 38$.

Corynolophus reinhardti Gill, 1878, Proc. U. S. Nat. Mus., 1: 228; Goode and Bean, 1895, Ocean. Ichth., p. 494 ; Jordan and Evermann, 1898, Bull. U. S. Nat. Mus., 47: 2733; Tanaka, 1908, Jour. Coll. Sci. Imp. Univ. Tokyo, 23, (13), p. 22, pl. 1, fig. 5; 1931, Jour. Fac. Sci. Imp. Univ. Tokyo, Zool., 3, (1), p. 53; Jordan, Tanaka, and Snyder, 1913, Jour. Coll. Sci,Imp. Univ. Tokyo, 33, (1), p. 427.

?Corynophorus compressus Osório, 1912, Mem. Mus. Bocage, Lisboa, 4: 89, figs. 1, 2; Nobre, 1935, Fauna Mar. Portugal, 1: 235, pl. 32, fig. 105, $c$, pl. 33, fig. 106.

Corynolophus sagamius Tanaka, 1918, Fig. Descr. Fishes Japan, 27: 491, pl. 134, fig. 377 .

Corynolophus globosus Tanaka, 1918, op. cit., 29: 529, pl. 139, fig. 388.

?Lipactis megalops Beebe, 1929, Zoologica, 12: 19.

Himantolophus danae Regan and Trewavas, 1932, Dana Rep., 2: 60, pl. 1, fig. 2, text figs. 87,88 .

Himantolophus globosus Barbour, 1942, Proc. New England Zool. Club, 21 :

82, pls. 12, 14; Bigelow and Barbour, 1944, op. cit., 23: 14.

Himantolophus kainarae Barbour, 1942, op. cit., 21 : 82.

Himantolophus ranoides Barbour, 1942, op. cit., p. 83, pls. 13, 14.

\section{SYNONYMY OF MALES}

Lipactis tumidus Regan, 1925, Ann. Mag. Nat. Hist., (9), 15: 566; 1926, Oceanogr. Rep. Danish Dana Exp. 1920-22, 2: 43 (part), pl. 12, fig. 2; ?Roule and Angel, 1930, Rés. Camp. Sci. Monaco, 79: 122, pl. 6, fig. 164; ?Norman, 1930, Disc. Rep., 2: 357; Regan and Trewavas, 1932, Dana Rep., 2: 61; Fowler, 1936, Bull. Amer. Mus. Nat. Hist., 70: 1349, fig. 567.

Rhynchoceratias brevirostris Regan, 1925, Ann. Mag. Nat. Hist., (9), 15: 566; 1926, Oceanogr. Rep. Danish Dana Exp. 1920-22, 2: 43, pl. 13, fig. 1, text fig. 25, a; Norman, 1930, Disc. Rep., 2: 357; Regan and Trewavas, 1932, Dana Rep., 2: 62.

Rhynchoceratias onchorhynchus Regan, 1925, Ann. Mag. Nat. Hist., (9), 15: 566; 1926, Oceanogr. Rep. Danish Dana Exp. 1920-22, 2: 44 (part), pl. 13, fig. 3, text fig. 25, c; Parr, 1930, Copeia, p. 131, fig. 5; Regan and Trewavas, 1932, Dana Rep., 2: 62.

?Rhynchoceratias altirostris Regan and Trewavas, 1932, op. cit., p. 62, fig. 90 .

Deep-abyssal records.-Western Atlantic, ca. $8^{\circ}$ N., $44^{\circ}$ W., 4000 meters, two male specimens. 
Eastern Atlantic, ca. $17^{\circ}$ N., $29^{\circ}$ W., 3000 meters, one young female specimen.

Western Atlantic, ca. $8^{\circ}$ N., $44^{\circ}$ W., 2500 meters, one male specimen.

Western Atlantic, ca. $17^{\circ}$ N., $64^{\circ}$ W., 2500 meters, one male specimen.

Distribution.-Eastern Atlantic from Iceland to South Africa. Western Atlantic from Greenland to off northern South America. Western Pacific, South China Sea and Japan. More than forty specimens known, some washed ashore or from an unknown depth, twenty-four recorded between 30 and 1500 meters, and five from deep-abyssal waters. Larvae have been taken in the western, eastern and southern Atlantic, off South Africa, in the northern Indian Ocean, and the western, southern and eastern Pacific.

Length. $-13-580 \mathrm{~mm}$.

\section{Himantolophus appeli Clarke}

Aegeonichthys appelii Clark, 1878, Trans. New Zealand Inst., 10: 245, pl. 6 -New Zealand.

Aegeonichthys appelii Günther, 1887, Rep. Sci. Res. Voy. Challenger, Zool., 22: 52; Goode and Bean, 1895, Ocean. Ichth., p. 494; Gill, 1908, Ann. Rep. Smithsonian Inst., p. 582, fig. 19; Waite, 1911, Trans. New Zealand Inst., 44: 194, pl. 10; Phillipps, 1927, New Zealand Mar. Dept. Fish. Bull., 1 : 55.

Himantolophus appelii Regan, 1926, Oceanogr. Rep. Danish Dana Exp. 1920-22, 2: 41; Regan and Trewavas, 1932, Dana Rep., 2: 60, fig. 89; Bertelsen, 1951, op. cit., 39: 66.

Distribution.-New Zealand. Only two specimens known, one washed ashore, the other caught on a line, depth unknown.

Length. $-310-410 \mathrm{~mm}$.

\section{Himantolophus azurlucens Beebe and Crane}

Himantolophus azurlucens Beebe and Crane, 1947, Zoologica, 31 : 155, pl. 1, text figs. 3, 4-Pacific off Panama, ca. $7^{\circ} \mathrm{N} ., 79^{\circ} \mathrm{W}$.

Himantolophus azurlucens Bertelsen, 1951, Dana Rep., 39: 66.

Distribution.-Eastern Pacific, only the type known, 914 meters. Length.-123 mm.

\section{Himantolophus rostratus Regan}

Rhynchoceratias rostratus Regan, 1925, Ann. Mag. Nat. Hist., (9), 15: 567 - Gulf of Panama.

Rhynchoceratias rostratus Regan, 1926, Oceanogr. Rep. Danish Dana Exp. 1920-22, 2: 44 (part), pl. 13, fig. 4, text fig. 25; Regan and Trewavas, 1932, Dana Rep., 2: 62 . 
Himantolophus rostratus Bertelsen, 1951, op. cit., 39: 55, 60, figs. 25, $d, e, 26, f$.

Deep-abyssal records and distribution.-Eastern Pacific, 2700 meters, the type.

Eastern Atlantic, ca. $17^{\circ}$ N., $24^{\circ}$ W., 2000 meters, one specimen.

Length.-38-46 mm.

Remarks.-Both specimens are males and may belong to different species. The Atlantic example, $46 \mathrm{~mm}$., is the largest known free-living male ceratioid.

\section{Family DICERATIIDAE}

Two genera and three species are contained in this family; a third genus, Laevoceratias, based on a single male specimen, is retained until further material can connect it with one of the other genera.

\section{Diceratias bispinosus Günther}

Ceratias (Diceratias) bispinosus Günther, 1887, Rep. Sci. Res. Voy. Challenger, Zool., 22: 53, pl. 11, fig. B-Banda Sea, ca. $4^{\circ}$ S., $129^{\circ}$ E., 658 meters.

Ceratias bispinosus Alcock, 1896, Jour. Asiat. Soc. Bengal, 65: 318; Alcock and McArdle, 1900, Ill. Zool. Investigator, Fishes, pl. 35, fig. 2.

Ceratias (Diceratias) bispinosus Alcock, 1899, Descr. Cat. Indian Deep-sea Fishes, p. 56.

Diceratias bispinosus Regan, 1926, Oceanogr. Rep. Danish Dana Exp. 1920-22, 2: 42, fig. 24; Regan and Trewavas, 1932, Dana Rep., 2: 31, 58, figs. 29, $a$, 85, $a$; Bertelsen, 1951, op. cit., 39:67, 69, figs. 27, 28.

Distribution.-Western Pacific in the Banda Sea. North Indian Ocean, Laccadive Sea. Two specimens, 658 and 1163 meters. Two larvae have been recorded, one from the Celebes Sea and one from the north Indian Ocean.

Length. $-10-140 \mathrm{~mm}$.

Remarks.-Males unknown.

\section{Paroneirodes glomerosus Alcock}

Paroneirodes glomerosus Alcock, 1890, Ann. Mag. Nat. Hist., (6), 6: 206, pl. 9, fig. 6-Bay of Bengal, ca. $15^{\circ}$ N., $81^{\circ} \mathrm{E}$.

Paroneirodes glomerosus (glomerulosus of authors) Goode and Bean, 1895, Ocean. Ichth., p. 493; Alcock, 1896, Jour. Asiat. Soc. Bengal, 65: 318; Gill, 1908, Ann. Rep. Smithsonian Inst., p. 579, fig. 15; Norman, 1930, Disc. Rep., 2: 356, fig. 46; Regan and Trewavas, 1932, Dana Rep., 2: 58; Bertelsen, 1951, op. cit., 39: 70. 
Oneirodes glomerosus Alcock, 1899, Descr. Cat. Indian Deep-sea Fishes, p. 57; 1902, Nat. Indian Seas, p. 236, fig. 32; Alcock and McArdle, 1900, Ill. Zool. Investigator, Fishes, pl. 28, fig. 4.

Diceratias glomerulosus Regan, 1926, Oceanogr. Rep. Danish Dana Exp. 1920-22, 2: 42; Fowler, 1936, Bull. Amer. Mus. Nat. Hist., 70: 1344, fig. 563 .

Deep-abyssal record.-North Indian Ocean, 2304 meters, the type.

Distribution.-North Indian Ocean. Southeastern Atlantic, ca. $15^{\circ} \mathrm{S} ., 10^{\circ} \mathrm{E}$. Only two specimens known, one deep-abyssal, the other in $600-700$ meters.

Length.-26-28 mm.

Remarks.-Males and larvae unknown.

\section{Paroneirodes wedli Pietschmann}

Phrynichthys wedli Pietschmann, 1926, Anz. Akad. Wiss. Wien, 63, (11), p. 89-Madeira.

Phrynichthys wedli Pietschmann, 1930, Ann. Nat. Mus. Wien, 44: 419, fig.; Fowler, 1936, Bull. Amer. Mus. Nat. Hist., 70: 1344, fig. 564.

Paroneirodes wedli Regan and Trewavas, 1932, Dana Rep., 2: 58; Bertelsen, 1951, op. cit., 39: 70.

Distribution.-Eastern Atlantic, known only from the type. Depth of capture unknown.

Length. $-55 \mathrm{~mm}$.

Remarks.-Perhaps not distinct from P. glomerosus. Males and larvae unknown.

\section{Laevoceratias liparis Parr}

Laevoceratias liparis Parr, 1927, Bull. Bingham Oceanogr. Coll., 3, (1), p. 33, fig. 13-off the Bahama Islands.

Laevoceratias liparis Regan and Trewavas, 1932, Dana Rep., 2: 93; Bertelsen, 1951, op. cit., 39: 70, fig. 29.

Distribution.-Western Atlantic. Known only from the type, a male specimen taken at a depth of 1609 meters.

Length. $-23 \mathrm{~mm}$.

\section{Family ONEIRODIDAE}

The wealth of material assigned to the family Oneirodidae has been separated by Bertelsen (1951) into fifteen genera and about forty species. As with all ceratioids, variability and ontogenetic changes in appearance greatly complicate the taxonomy of the 
group. In some instances, described species of the genera Oneirodes and Chaenophryne can neither be separated nor synonymized, with present knowledge. Bertelsen has referred to these as "groups" of species and this procedure is followed in the present paper. Parasitic males are not known and Bertelsen reported evidence that they are probably free-living throughout life, attaching to the female temporarily only.

\section{Oneirodes cristatus Regan and Trewavas}

Dolopichthys cristatus Regan and Trewavas, 1932, Dana Rep., 2: 67, fig. 93Banda Sea, ca. $5^{\circ}$ S., $131^{\circ}$ E., 2500 meters.

Oneirodes cristatus Bertelsen, 1951, op. cit., 39: 79, fig. 31, c, $d$.

Deep-abyssal records and distribution.--Western Pacific, Banda Sea, 4000 meters, 2500 meters, two specimens.

Western Pacific north of Celebes, ca. $3^{\circ}$ N., $123^{\circ}$ E., 3500 meters, one specimen.

Length. - 30-215 mm.

Remarks.-Males and larvae unknown.

\section{Oneirodes eschrichti group. Table 27.}

Oneirodes eschrichtii Lütken, *1871, Overs. Dansk Vidensk. Selsk. Forhandl., p. 56, pl. 2-off Greenland.

Oneirodes eschrichtii Lütken, 1878, Dansk Vidensk. Selsk. Skr., (5), 11, (5), pl. 2 , fig. 6; Gill, 1878, Proc. U. S. Nat. Mus., 1: 218; Günther, 1887, Rep. Sci. Res. Voy. Challenger, Zool., 22: 56; Goode and Bean, 1895, Ocean. Ichth., p. 492; Jordan and Evermann, 1898, Bull. U. S. Nat. Mus., 47: 2732; Ehrenbaum, 1902, Fauna Arct., 2: 76; Regan, 1926, Oceanogr. Rep. Danish Dana Exp. 1920-22, 2: 26 (part), fig. 17; Regan and Trewavas, 1932, Dana Rep., 2: 63; Fowler, 1936, Bull. Amer. Mus. Nat. Hist., 70: 1140, fig. 479; Maul, 1949, Bol. Mus. Mun. Funchal, 4, (9), p. 34, figs. 14-17.

Oneirodes megaceros Holt and Byrne, 1908, Ann. Mag. Nat. Hist., (8), 1: 93; 1908, Rep. Fish. Ireland 1906, Sci. Invest., 5: 60; Murray and Hjort, 1912, Depths of Ocean, pp. 94, 614, fig. 81; Fowler, 1936, Bull. Amer. Mus. Nat. Hist., 70 : 1339.

?Dermatias platynogaster Radcliffe, 1912, Proc. U. S. Nat. Mus., 42: 206, pl. 17 , fig. 3 .

Dolopichthys anisacanthus Regan, 1925, Ann. Mag. Nat. Hist., (9), 15: 562; Regan and Trewavas, 1932, Dana Rep., 2: 72, pl. 2, fig. 2, text fig. 105.

Dolopichthys heteracanthus Regan, 1926, Oceanogr. Rep. Danish Dana Exp. 1920-22, 2 : 28 (part).

Dolopichthys megaceros Regan, 1926, op. cit., p. 29 (part); Regan and Trewavas, 1932, Dana Rep., 2: 67, 71, fig. 103; Koefoed, 1944, Rep. Sci. Res. M. 
Sars No. Atl. Deep-sea Exp. 1910, 4, (2), p. 6, pl. 1, figs. 4, $a, b, 5$, pl. 3, fig. 6 .

?Dolopichthys platynogaster Regan, 1926, Oceanogr. Rep. Danish Dana Exp. 1920-22, 2: 29; Regan and Trewavas, 1932, Dana Rep., 2: 68, fig. 95.

Dolopichthys sp. Regan, 1926, Oceanogr. Rep. Danish Dana Exp. 1920-22, 2: 5; Norman, 1939, Sci. Rep. John Murray Exp. 1933-34, 7, (1), p. 115, figs. 40,41 .

?Dolopichthys (Dermatias) platynogaster Parr, 1927, Bull. Bingham Oceanogr. Coll., 3, (1), p. 14.

Dolopichthys obtusus Parr, 1927, op. cit., p. 16, fig. 5; Regan and Trewavas, 1932, Dana Rep., 2: 71.

Dolopichthys tentaculatus Beebe, 1932, Zoologica, 13: 88, fig. 23; 1937, op. cit., 22: 207; Regan and Trewavas, 1932, Dana Rep., 2: 70; Hollister and Beebe, 1934, Bull. N. Y. Zool. Soc., 37: 188, fig.

Dolopichthys digitatus Regan and Trewavas, 1932, Dana Rep., 2: 68, fig. 94.

Dolopichthys simplex Regan and Trewavas, 1932, op. cit., p. 68, fig. 96.

Dolopichthys pollicifer Regan and Trewavas, 1932, op. cit., p. 69, fig. 97.

Dolopichthys diadematus Regan and Trewavas, 1932, loc. cit., fig. 98.

Dolopichthys brevifilis Regan and Trewavas, 1932, loc. cit., fig. 99.

Dolopichthys pennatus Regan and Trewavas, 1932, loc. cit., fig. 100 .

Dolopichthys frondosus Regan and Trewavas, 1932, op. cit., p. 70, fig. 101.

Dolopichthys cirrifer Regan and Trewavas, 1932, loc. cit., fig. 102.

Dolopichthys plumatus Regan and Trewavas, 1932, op. cit., p. 71, fig. 104.

Dolopichthys heteronema Regan and Trewavas, 1932, op. cit., p. 72, fig. 106.

Dolopichthys ptilotus Regan and Trewavas, 1932, op. cit., p. 73, fig. 107.

Dolopichthys multifilis Regan and Trewavas, 1932, loc. cit., fig. 108.

Dolopichthys claviger Regan and Trewavas, 1932, loc. cit., fig. 109.

?Dolopichthys hibernicus Fraser-Brunner, 1935, Proc. Roy. Irish Acad., B42: 325 , fig. 4 .

?Oneirodes theodor-tissieri Belloc, 1938, Rev. Trav. Pêches Marit., 11: 303, figs. $23,25,26$.

Oneirodes bulbosus Chapman, 1939, Proc. U. S. Nat. Mus., 86: 538, fig. 70; Clemens and Wilby, 1946, Bull. Fish. Res. Bd. Canada, 68: 338, fig. 253.

Oneirodes eschrichtii group Bertelsen, 1951, Dana Rep., 39: 79, figs. 31, e-g, 32-37.

\section{SYNONYMY OF MALES}

Rhynchoceratias leucorhinus Regan, 1926, Oceanogr. Rep. Danish Dana Exp. 1920-22, 2: 44 (part).

Caranactis pumilus Regan and Trewavas, 1932, Dana Rep., 2: 59, fig. 86.

Trematorhynchus leucorhinus Regan and Trewavas, 1932, op. cit., p. 91 (part).

Deep-abyssal records.-Eastern Pacific, Gulf of Panama and ca. $0^{\circ} 18^{\prime} \mathrm{S} ., 99^{\circ} \mathrm{W}$., 2000 meters, five specimens from five hauls. 
Western Pacific in the Sulu Sea and north of New Guinea, 25002000 meters, four specimens in four hauls.

North Indian Ocean near Ceylon, ca. $5^{\circ} \mathrm{N} ., 80^{\circ}$ E., 2500 meters, one specimen.

Southeastern Atlantic, ca. $15^{\circ}$ S., $5^{\circ}$ W., 2000 meters, one specimen.

Eastern Atlantic, ca. $25^{\circ}$ N., $20^{\circ}$ W., 3500 meters, one specimen.

North Atlantic, $8^{\circ}-32^{\circ} \mathrm{N}$., $17^{\circ}-65^{\circ} \mathrm{W}$., $2500-2000$ meters, nine specimens from eight hauls.

Distribution.-Atlantic from Greenland and ca. $53^{\circ}$ N., $12^{\circ} \mathrm{W}$. south to ca. $8^{\circ} \mathrm{N} ., 44^{\circ} \mathrm{W}$. and $15^{\circ} \mathrm{S} ., 5^{\circ} \mathrm{W}$. Eastern Pacific from the Gulf of Panama to ca. $0^{\circ} 18^{\prime}$ S., $99^{\circ} \mathrm{W}$. (?) Northeastern Pacific off the Queen Charlotte Islands $(O$. bulbosus Chapman, included here with some doubt). Western Pacific around the Dutch East Indies and the Philippines. Indian Ocean south of Ceylon and in the Gulf of Aden. Forty-two specimens in all, twenty-one of them deep-abyssal, two from an unknown depth (including the type), the rest between 300 and 1700 meters.

The Dana took 163 larvae in the eastern, western and southeastern Atlantic, the eastern and western Pacific, and the north Indian Ocean.

Length. $-14-227 \mathrm{~mm}$.

Remarks.-Many or all of the species included in this group may be synonymous.

\section{Oneirodes flagellifer group}

Dolopichthys flagellifer Regan and Trewavas, 1932, Dana Rep., 2: 74, fig. 111 -Indian Ocean near Ceylon, ca. $5^{\circ} \mathrm{N} ., 80^{\circ} \mathrm{E}$.

Dolopichthys thysanophorus Regan and Trewavas, 1932, op. cit., p. 74, fig. 110.

Oneirodes flagellifer group Bertelsen, 1951, op. cit., 39: 84, figs. $31, j, k$.

Deep-abyssal records and distribution.-North Indian Ocean, 2200 meters, the type.

Western Pacific, Sulu Sea, 2200 meters, two specimens in one haul.

Length.-17-32 mm.

Remarks.-Males and larvae unknown.

\section{Oneirodes schmidti group}

Dolopichthys schmidti Regan and Trewavas, 1932, Dana Rep., 2: 75, fig. 113 -Banda Sea. 
Dolopichthys macronema Regan and Trewavas, 1932, op. cit., p. 66, fig. 91 .

Dolopichthys mirus Regan and Trewavas, 1932, op. cit., p. 74, fig. 112.

Oneirodes schmidti group Bertelsen, 1951, op. cit., 39: 84, fig. $3, h, i$.

Deep-abyssal records.-Western Pacific, 3500 meters, the type. North Indian Ocean, ca. $1^{\circ}$ N., $96^{\circ}$ E., 2000 meters, one specimen.

Distribution.-Western Pacific and north Indian Ocean, two deep-abyssal records. Western Atlantic, ca. $17^{\circ} \mathrm{N} ., 64^{\circ} \mathrm{W}$, one specimen, 300-500 meters.

Length. $-37-59 \mathrm{~mm}$.

Remarks.-Males and larvae unknown.

\section{Oneirodes inimicus Fraser-Brunner}

Dolopichthys inimicus Fraser-Brunner, 1935, Proc. Roy. Irish Acad., B42: 324 , fig. 3 -Atlantic, ca. $53^{\circ}$ N., $13^{\circ} \mathrm{W}$.

Oneirodes inimicus Bertelsen, 1951, Dana Rep., 39: 85.

Distribution.-Eastern Atlantic off southwestern Ireland, one specimen, 320 meters.

Length. $-33 \mathrm{~mm}$.

Remarks.-Males and larvae unknown.

\section{Oneirodes acanthias Gilbert}

Monoceratias acanthias Gilbert, 1915, Proc. U. S. Nat. Mus., 48: 379, pl. 22, fig. 4-off Santa Cruz Island, southern California, 1397-1629 meters.

Dolopichthys acanthias Regan, 1926, Oceanogr. Rep. Danish Dana Exp. 1920-22, 2: 28; Regan and Trewavas, 1932, Dana Rep., 2: 76, fig. 114; Schultz, 1934, Copeia, p. 66, figs. 1, 2; Bolin and Myers, 1950, Stanf. Ichth. Bull., 3: 203-208.

Dolopichthys (Monoceratias) acanthias Parr, 1927, Bull. Bingham Oceanogr. Coll., 3, (1), p. 15.

Dolopichthys thompsoni Schultz, 1934, Copeia, p. 66, figs. 3, 4.

Oneirodes acanthias Bertelsen, 1951, Dana Rep., 39: 85, fig. 38.

Distribution.-Eastern Pacific off southern California, three specimens, 900 meters and 1397-1629 meters. North Pacific, ca. $54^{\circ} \mathrm{N}$., $159^{\circ} \mathrm{W}$, , one specimen in 900 meters.

Length. $-32-80 \mathrm{~mm}$.

Remarks.-Males and larvae unknown.

\section{Oneirodes carlsbergi Regan and Trewavas}

Dolopichthys carlsbergi Regan and Trewavas, 1932, Dana Rep., 2: 76, fig. 115 Gulf of Panama, ca. 500 meters. 
Oneirodes eschrichtii Regan, 1926, Oceanogr. Rep. Danish Dana Exp. 1920-22, 2: 26 (part).

Dolopichthys heteracanthus Regan, 1926, op. cit., p. 28 (part); Norman, 1930, Disc. Rep., 2 : 353 (part).

Oneirodes carlsbergi Bertelsen, 1951, Dana Rep., 39: 86, figs. 31, m-o, 39.

Deep-abyssal records.-Eastern Atlantic, ca. $15^{\circ}$ N., $26^{\circ}$ W., 2000 meters, one specimen.

Mid-Atlantic, ca. $12^{\circ} \mathrm{N}$., $35^{\circ}$ W., 2000 meters, one specimen.

Distribution.-Eastern Atlantic off Cape Verde and the Cape Verde Islands, one deep-abyssal specimen and two in 900 meters. Mid-Atlantic, one deep-abyssal record. Eastern Pacific in the Gulf of Panama and ca. $2^{\circ} \mathrm{N}$., $87^{\circ} \mathrm{W}$., three specimens, 500-1700 meters. Western Pacific, ca. $16^{\circ} \mathrm{N}$., $120^{\circ}$ E., one specimen in ca. $300-500$ meters.

Length. -25-205 mm.

Remarks.-Males and larvae unknown.

\section{Oneirodes luetkeni Regan}

Dolopichthys luetkeni Regan, 1925, Ann. Mag. Nat. Hist., (9), 15: 562-Gulf of Panama.

Dolopichthys heteracanthus Regan, 1925, loc. cit.; 1926, Oceanogr. Rep. Danish Dana Exp. 1920-22, 2: 28 (part), pl. 5, fig. 1; Regan and Trewavas, 1932, Dana Rep., 2: 69, 77, fig. 117 .

Dolopichthys luetkeni Regan, 1926, Oceanogr. Rep. Danish Dana Exp. 1920-22, 2: 27, pl. 4, fig. 2; Parr, 1927, Bull. Bingham Oceanogr. Coll., 3, (1), p. 15; Regan and Trewavas, 1932, Dana Rep., 2: 76, fig. 116; Beebe and Crane, 1947, Zoologica, 31 : 159.

Oneirodes luetkeni Bertelsen, 1951, Dana Rep., 39: 86, figs. 31, $p-s, 40$.

Deep-abyssal records.-Gulf of Panama, 2000-2500 meters, five specimens from four hauls.

Distribution.-Eastern Pacific in the Gulf of Panama, off Costa Rica, and near Cocos Island, twenty-six specimens known, five of them deep-abyssal, the others between $300-500$ and 1700 meters.

Length. $-16-160 \mathrm{~mm}$.

Remarks.-Males and larvae unknown.

\section{Oneirodes melanocauda Bertelsen}

Oneirodes melanocauda Bertelsen, 1951, Dana Rep., 39: 87, figs. 31, l, $41-$ South China Sea, ca. $6^{\circ}$ N., $114^{\circ}$ E.

Deep-abyssal record.-Western Pacific, 2500 meters, the type. 
Distribution.-Western Pacific, one deep-abyssal record. Three larvae are known, one from the western Pacific, one from the north Indian Ocean and one from the western Atlantic.

\section{Length. $-8-21 \mathrm{~mm}$.}

Remarks.-The type is a metamorphosing female. Adolescent and adult stages are unknown.

Microlophichthys microlophus Regan. Table 28.

Dolopichthys microlophus Regan, 1925, Ann. Mag. Nat. Hist., (9), 15 : 563 Atlantic, ca. $17^{\circ} \mathrm{N} ., 24^{\circ} \mathrm{W}$.

Dolopichthys heteracanthus Regan, 1926, Oceanogr. Rep. Danish Dana Exp. 1920-22, 2: 28 (part).

Dolopichthys allector Regan, 1926, op. cit., p. 28 (part).

Dolopichthys microlophus Regan, 1926, op. cit., p. 29, fig. 18; Regan and Trewavas, 1932, Dana Rep., 2: 77, figs. 118, 119.

Dolopichthys analogus Parr, 1927, Bull. Bingham Oceanogr. Coll., 3, (1), pp. 16 , 20, fig. 7; Regan and Trewavas, 1932, Dana Rep., 2:78, fig. 120; Beebe, 1937, Zoologica, 22: 207.

Dolopichthys exiguus Regan and Trewavas, 1932, Dana Rep., 2: 78, fig. 121.

Dolopichthys implumis Regan and Trewavas, 1932, loc. cit., fig. 122; Beebe and Crane, 1947, Zoologica, 31 : 160, fig. 5.

Microlophichthys microlophus Bertelsen, 1951, Dana Rep., 39: 89, 90, figs. 42-45.

\section{SYNONYMY OF MALES}

Rhynchoceratias leucorhinus Regan, 1926, Oceanogr. Rep. Danish Dana Exp. 1920-22, 2: 44 (part).

Deep-abyssal records.-Eastern Atlantic, $0^{\circ} 31^{\prime}$ S., $11^{\circ} 02^{\prime} \mathrm{W}$., 4000 meters, one specimen.

Eastern Atlantic off the Cape Verde Islands, 2000 meters, the type.

Western Atlantic off Bermuda and in the Caribbean Sea, 2000, 2012 meters, two specimens.

Gulf of Panama, 2000-2200 meters, five specimens in four hauls.

Western Pacific, north of New Guinea, ca. $3^{\circ}$ N., $137^{\circ}$ E., 2500 meters, one specimen.

Western Pacific, Banda Sea, ca. $4^{\circ}$ N., $128^{\circ}$ E., 2000 meters, one specimen.

Indian Ocean, ca. $5^{\circ} \mathrm{N} ., 90^{\circ}$ E., 2000 meters, one specimen.

Distribution.--Eastern Atlantic off Cape Verde Islands and in ca. $0^{\circ}$ S., $11^{\circ} \mathrm{W}$. Western Atlantic off Bermuda and in the Caribbean Sea. Eastern Pacific in the Gulf of Panama, off Costa Rica 
and near the Galapagos Islands. Western Pacific around the Dutch East Indies and in the South China Sea (ca. $15^{\circ}$ N., $115^{\circ}$ E.). North Indian Ocean between Sumatra and Ceylon. Twenty-one specimens in all, twelve of them deep-abyssal, the others in 914-1800 meters. Larvae known from the eastern, southeastern and western Atlantic, the eastern Pacific and the north Indian Ocean.

Length. $-2.4-62 \mathrm{~mm}$.

\section{Microlophichthys andracanthus Bertelsen}

Microlophichthys andracanthus Bertelsen, 1951, Dana Rep., 39: 92, fig. 47Caribbean Sea, ca. $17^{\circ} \mathrm{N} ., 64^{\circ} \mathrm{W}$.

Deep-abyssal record and distribution.-Western Atlantic, 2700 meters, the type, an adult male.

Length. $-24 \mathrm{~mm}$.

Remarks.-Females and larvae unknown.

\section{Tyrannophryne pugnax Regan and Trewavas}

Tyrannophryne pugnax Regan and Trewavas, 1932, Dana Rep., 2: 83, pl. 4, fig. 1 -Pacific, ca. $18^{\circ} \mathrm{S} ., 153^{\circ} \mathrm{W}$.

Tyrannophryne pugnax Bertelsen, 1951, op. cit., 39: 93, fig. 48.

Deep-abyssal record and distribution.-South Pacific near Tahiti, 2500 meters, one specimen.

Length. $-18.5 \mathrm{~mm}$.

Remarks.-Males and larvae unknown.

\section{Leptacanthichthys gracilispinis Regan}

Dolopichthys gracilispinis Regan, 1925, Ann. Mag. Nat. Hist., (9), 15: 563Gulf of Panama.

Dolopichthys gracilispinis Regan, 1926, Oceanogr. Rep. Danish Dana Exp. 1920-22, 2: 30, pl. 5, fig. 2; Regan and Trewavas, 1932, Dana Rep., 2: 80 , fig. 128.

Leptacanthichthys gracilispinis Bertelsen, 1951, op. cit., 39: 94, fig. 49.

Deep-abyssal records and distribution.-Eastern Pacific, 2200 meters, the type.

Western Atlantic, ca. $28^{\circ} \mathrm{N}$., $56^{\circ} \mathrm{W}$., 2000 meters, one specimen. Length. $-50-65 \mathrm{~mm}$.

Remarks.-Males and larvae unknown.

\section{Chirophryne xenolophus Regan and Trewavas}

Chirophryne xenolophus Regan and Trewavas, 1932, Dana Rep., 2: 82, figs. 131,132 - western Pacific, ca. $14^{\circ}$ N., $119^{\circ}$ E. 
Chirophryne xenolophus Bertelsen, 1951, op. cit., 39: 94, fig. 50.

Distribution.-Western Pacific, South China Sea, 1700 meters, type only.

Length. $-15 \mathrm{~mm}$.

Remarks.-Males and larvae unknown.

\section{Ctenochirichthys longimanus Regan and Trewavas}

Ctenochirichthys longimanus Regan and Trewavas, 1932, Dana Rep., 2: 82, pl. 3, fig. 3-Gulf of Panama.

Dolopichthys heteracanthus Regan, 1926, Oceanogr. Rep. Danish Dana Exp. 1920-22, $2: 7$ (part).

Ctenochirichthys longimanus Bertelsen, 1951, Dana Rep., 39: 95, figs. 51, 52.

\section{SYNONYMY OF MALES}

Trematorhynchus multiradiatus Beebe and Crane, 1947, Zoologica, 31: 166, fig. 11.

Deep-abyssal record.-Gulf of Panama, 2000 meters, the type.

Distribution.-Eastern Pacific, Gulf of Panama, two specimens, 1700 and 910 meters, and one deep-abyssal specimen. Two male larvae known, one in the Gulf of Panama and one from the eastern Atlantic.

Length. $-6.5-47 \mathrm{~mm}$.

\section{Dolopichthys sp.}

Rhynchoceratias leucorhinus Regan, 1926, Oceanogr. Rep. Danish Dana Exp. 1920-22, 2: 44 (part).

Dolopichthys sp. Regan and Trewavas, 1932, Dana Rep., 2: 9, 10; Bertelsen, 1951, op. cit., 39: 98, figs. 54, a, 55, 56.

Distribution.-Eastern and western Atlantic. Eastern, southern and western Pacific. North Indian Ocean. Indian Ocean off South Africa.

Length. $-2.4-17.3 \mathrm{~mm}$.

Remarks.-Under this name Bertelsen (1951) listed the Dolopichthys larval and male specimens that cannot be separated into species.

\section{Dolopichthys allector Garman}

Dolopichthys allector Garman, 1899, Mem. Mus. Comp. Zool., 24: 81, pls. 13-15 -Pacific off Panama, ca. $5^{\circ}$ N., $86^{\circ} \mathrm{W}$.

Dolopichthys allector Gill, 1908, Ann. Rep. Smithsonian Inst., p. 580, fig. 16; Regan and Trewavas, 1932, Dana Rep., 2: 78, 80; (?) Beebe and Crane, 1947, Zoologica, 31 : 161, fig. 7; Bertelsen, 1951, Dana Rep., 39: 100. 
Distribution.-Eastern Pacific, the type in 1409 meters and one specimen, perhaps belonging to this species, taken near the Galapagos Islands in 823 meters.

Length. $-25 \mathrm{~mm}$. and $72 \mathrm{~mm}$. (type).

Remarks.-Males and larvae unknown.

\section{Dolopichthys danae Regan}

Dolopichthys danae Regan, 1926, Oceanogr. Rep. Danish Dana Exp. 1920-22, 2: 29 , pl. 4, fig. 1 -Atlantic, ca. $8^{\circ} \mathrm{N} ., 44^{\circ} \mathrm{W}$.

Dolopichthys danae Regan and Trewavas, 1932, Dana Rep., 2: 80, fig. 127; Bertelsen, 1951, op. cit., 39: 96, 98, 100, figs. 53, f, 54, $d$.

Deep-abyssal record and distribution.-Eastern Atlantic, 4000 meters, type only.

Length. $-95 \mathrm{~mm}$.

Remarks.-Males and larvae unknown.

\section{Dolopichthys niger Brauer}

Dolopichthys niger Brauer, 1902, Zool. Anz., 25: 292-Indian Ocean, ca. $29^{\circ}$ S., $89^{\circ} \mathrm{E}$.

Oneirodes niger Brauer, 1906, Wiss. Ergebn. Deutschen Tiefsee Exp. Valdivia, 15, (1), p. 316 , pl. 15, fig. 6 ; 1908, op. cit., 15, (2), pp. 103, 184, pl. 32, figs. 7,8 , pl. 34 , figs. 15,16 , pl. 44 , figs. 2,3 .

Dolopichthys niger Regan, 1926, Oceanogr. Rep. Danish Dana Exp. 1920-22, 2: 27; Parr, 1927, Bull. Bingham Oceanogr. Coll., 3, (1), p. 14; Regan and Trewavas, 1932, Dana Rep., 2: 79, fig. 125; Bertelsen, 1951, op. cit., 39: 96,100 , fig. $53, a$.

Deep-abyssal records and distribution.-South Indian Ocean, 2500 meters, the type.

North Indian Ocean near Zanzibar, ca. $4^{\circ}$ S., $48^{\circ}$ E., 2000 meters, one specimen.

Length.-13-15 mm.

Remarks.-Males and larvae unknown.

\section{Dolopichthys cornutus Gilchrist and von Bonde}

Oneirodes cornutus Gilchrist and von Bonde, 1924, Rep. Fish. Mar. Biol. Surv.

So. Afr., 3, (7), p. 23, pl. 6, fig. 2-South Africa, exact locality unknown.

Dolopichthys cornutus Regan, 1926, Oceanogr. Rep. Danish Dana Exp. 192022, 2: 27 ; Parr, 1927, Bull. Bingham Oceanogr. Coll., 3, (1), p. 14; Barnard, 1927, Ann. So. Afr. Mus., 21: 1004; Regan and Trewavas, 1932, Dana Rep., 2: 63; Smith, 1949, Sea Fishes So. Afr., p. 429, fig. 1231.

Distribution.- South Africa, one specimen, depth of capture unknown. 
Length. $-16.5 \mathrm{~mm}$.

Remarks.-Males and larvae unknown.

\section{Dolopichthys longicornis Parr}

Dolopichthys longicornis Parr, 1927, Bull. Bingham Oceanogr. Coll., 3, (1), p. 18, fig. 6-Bahama Islands.

Dolopichthys megaceros Regan, 1926, Oceanogr. Rep. Danish Dana Exp. 1920-22, $2: 29$ (part).

Dolopichthys danae Regan, 1926, loc. cit. (part).

Dolopichthys longicornis Norman, 1930, Disc. Rep., 2: 354; Regan and Trewavas, 1932, Dana Rep., 2: 79; Beebe, 1937, Zoologica, 22: 207; Bertelsen, 1951, Dana Rep., 39: 96, 98, 100, figs. 53, b-e, 54, $c-f, 57$.

Dolopichthys pullatus Regan and Trewavas, 1932, op. cit., 2: 79, pl. 3, fig. 1, text fig. 123; Beebe and Crane, 1947, Zoologica, 31 : 161, fig. 6.

Dolopichthys mucronatus Regan and Trewavas, 1932, Dana Rep., 2: 79, fig. 124.

Dolopichthys jubatus Regan and Trewavas, 1932, op. cit., pp. 79, 80, fig. 126.

Deep-abyssal records.-Banda Sea, ca. $2^{\circ}$ S., $126^{\circ}$ E., 3500 meters, one specimen.

South China Sea, ca. $19^{\circ}$ N., $120^{\circ}$ E., 2500 meters, one specimen.

Eastern Atlantic, ca. $33^{\circ}$ N., $16^{\circ} \mathrm{W}$. and ca. $36^{\circ}$ N., $26^{\circ} \mathrm{W}$, 2000 meters, two specimens.

Western Atlantic, 2012 meters, the type.

Distribution.-Eastern Atlantic from near the Azores and Madeira to ca. $2^{\circ}$ S., $9^{\circ}$ W., two deep-abyssal records and two specimens in 800-1000 meters. Western Atlantic off Bermuda and the Bahamas, one deep-abyssal specimen and two from 1645 and 1829 meters. Eastern Pacific, Gulf of Panama, one specimen, 914 meters. Western Pacific, two deep-abyssal records. North Indian Ocean near the Seychelles, ca. $1^{\circ}$ S., $62^{\circ}$ E., one specimen in 1700 meters. Eleven specimens in all.

Length.-23-114 mm.

Remarks.-Most of the larval and male specimens listed as Dolopichthys spp. probably belong to this species (Bertelsen, 1951, p. 98).

\section{Danaphryne nigrifilis Regan and Trewavas}

Dolopichthys nigrifilis Regan and Trewavas, 1932, Dana Rep., 2: 67, fig. $92-$ South China Sea, ca. $19^{\circ}$ N., $120^{\circ}$ E.

Dolopichthys albifilosa Waterman, 1939, Bull. Mus. Comp. Zool., 85: 89, figs. $5,6$.

Danaphryne nigrifilis Bertelsen, 1951, Dana Rep., 39: 102, figs. 58, 59. 
Deep-abyssal record.-Western Pacific, 2000 meters, the type.

Distribution.-Western Pacific, one deep-abyssal record. Western Atlantic, ca. $35^{\circ} \mathrm{N}$., $69^{\circ} \mathrm{W}$., one specimen, depth of capture unknown.

Length. - 36-45 mm.

Remarks.-Males and larvae unknown.

\section{Pentherichthys sp. Bertelsen}

Pentherichthys sp. Bertelsen, 1951, Dana Rep., 39: 103, figs. 61, 62.

Rhynchoceratias leucorhinus Regan, 1926, Oceanogr. Rep. Danish Dana Exp. 1920-22, 2: 44 (part).

Distribution.-Under this heading Bertelsen listed nineteen larval and four male specimens belonging to the genus Pentherichthys. These were taken by the Dana in the eastern and western Atlantic, the Gulf of Panama in the eastern Pacific, the western Pacific, and the north Indian Ocean.

Length. $-5.3-15 \mathrm{~mm}$.

\section{Pentherichthys atratus Regan and Trewavas}

Dolopichthys atratus Regan and Trewavas, 1932, Dana Rep., 2: 81, pl. 3, fig. 2, text fig. 129-Gulf of Panama, 1700 meters.

Dolopichthys allector Regan, 1926, Oceanogr. Rep. Danish Dana Exp. 1920-22, 2: 28 (part).

Dolopichthys atratus Beebe and Crane, 1947, Zoologica, 31 : 162.

Pentherichthys atratus Bertelsen, 1951, Dana Rep., 39: 105, fig. 63.

Deep-abyssal records.-Gulf of Panama, 2200, 2000 meters, two specimens.

Distribution.-Eastern Pacific in the Gulf of Panama, off Costa Rica and near Cocos Island, two deep-abyssal records and five specimens in $914-1700$ meters.

Length. - 23-41.7 mm.

Remarks.-Males and larvae unknown.

\section{Pentherichthys venustus Regan and Trewavas}

Dolopichthys venustus Regan and Trewavas, 1932, Dana Rep., 2: 81, fig. 130Atlantic, ca. $12^{\circ} \mathrm{N} ., 20^{\circ} \mathrm{W}$.

Dolopichthys allector Norman, 1930, Disc. Rep., 2: 353, fig. 43.

Pentherichthys venustus Bertelsen, 1951, Dana Rep., 39: 105.

Distribution.-Eastern Atlantic, type only, 250-300 meters.

Length. $-36 \mathrm{~mm}$.

Remarks.-Males and larvae unknown. 


\section{Lophodolus acanthognathus Regan}

Lophodolus acanthognathus Regan, 1925, Ann. Mag. Nat. Hist., (9), 15: 563 Atlantic, ca. $28^{\circ} \mathrm{N} ., 56^{\circ} \mathrm{W} ., 2000$ meters.

Oneirodes sp. Murray and Hjort, 1912, Depths of Ocean, p. 104, fig. 90.

Lophodolus acanthognathus Regan, 1926, Oceanogr. Rep. Danish Dana Exp. 1920-22, 2: 30, pl. 6, fig. 1; Norman, 1930, Disc. Rep., 2: 354; Regan and Trewavas, 1932, Dana Rep., 2: 83; Parr, 1934, Bull. Bingham Oceanogr. Coll., 4, (6), p. 7; Beebe, 1937, Zoologica, 22: 207; Nybelin, 1948, Göteborgs K. Vetensk. Vitt.-Samh. Handl., (B), 5, (16), p. 86, fig. 9; Bertelsen, 1951, Dana Rep., 39: 107, figs. 64, b-d, 65; Grey, 1955, Fieldiana, Zool., $37: 299$.

Lophodolus lyra Beebe, 1932, Zoologica, 13: 96, fig. 28; Koefoed, 1944, Rep. Sci. Res. M. Sars No. Atl. Deep-sea Exp. 1910, 4, (2), p. 7, pl. 3, fig. 3.

Lophodolus Gregory, 1933, Trans. Amer. Phil. Soc., 23: 402, figs. 274, 276, $a$.

Deep-abyssal records.-Mid-Atlantic, ca. $47^{\circ}$ N., $31^{\circ}$ W., 4000, 3500 meters, two specimens.

Eastern Atlantic, ca. $36^{\circ}$ N., $9^{\circ}$ W., (?) 2000 meters, one specimen, depth of capture uncertain.

Western Atlantic, ca. $34^{\circ} \mathrm{N}$., $70^{\circ} \mathrm{W}$., 2700 meters, one specimen.

Western Atlantic, ca. $28^{\circ} \mathrm{N}$., $56^{\circ} \mathrm{W}$., 2000 meters, one specimen.

South China Sea, ca. $6^{\circ}$ N., $118^{\circ}$ E., 2200 meters, one specimen.

Distribution.-Atlantic from ca. $48^{\circ} \mathrm{N} ., 49^{\circ} \mathrm{W}$. south to ca. $2^{\circ} \mathrm{S}$., $9^{\circ} \mathrm{W}$. in the east and, in the west, to the Bahama Islands, five deep-abyssal specimens and fifty-six in 800-1829 meters. Western Pacific, one deep-abyssal record and one specimen in the Celebes Sea, 1000-1500 meters. Five larvae known, from the north Atlantic only.

Length. $-10-70 \mathrm{~mm}$.

Remarks.-Metamorphosed males unknown.

\section{Lophodolus dinema Regan and Trewavas}

Lophodolus dinema Regan and Trewavas, 1932, Dana Rep., 2: 83, pl. 4, fig. 3 -South China Sea, ca. $19^{\circ}$ N., $120^{\circ}$ E.

Lophodolus dinema Bertelsen, 1951, op. cit., 39: 108, fig. 64, a.

Deep-abyssal record and distribution.-Western Pacific, 2000 meters, type only.

Length. $-54 \mathrm{~mm}$.

Remarks.-Males and larvae unknown.

\section{Lophodolus indicus Lloyd}

Lophodolus indicus Lloyd, 1909, Mem. Indian Mus., 2: 167, pl. 14, fig. 7Indian Ocean off Travancore. 
Lophodolus indicus Regan, 1926, Oceanogr. Rep. Danish Dana Exp. 1920-22, 2: 30; Regan and Trewavas, 1932, Dana Rep., 2: 83; Bertelsen, 1951, op. cit., 39: 108 .

Distribution.-North Indian Ocean, type only, 1624 meters.

Length. $-65 \mathrm{~mm}$.

Remarks.-Males and larvae unknown.

\section{Chaenophryne longiceps group}

Himantolophus groenlandicus Regan, 1926, Oceanogr. Rep. Danish Dana Exp. 1920-22, 2: 40 (part).

Rhynchoceratias leucorhinus Regan, 1926, op. cit., p. 44 (part).

Trematorhynchus leucorhinus Regan and Trewavas, 1932, Dana Rep., 2: 91 (part).

Chaenophryne longiceps group Bertelsen, 1951, op. cit., 39: 110, figs. 68-70.

Distribution.-Eastern and western Atlantic. North Indian Ocean.

Length. $-3.2-30 \mathrm{~mm}$.

Remarks.-Under this heading Bertelsen discussed the larvae, males, and metamorphosing stages of females of the species Chaenophryne longiceps, C. bicornis, C. quadrifilis, C. crenata and C. crossota.

\section{Chaenophryne longiceps Regan}

Chaenophryne longiceps Regan, 1925, Ann. Mag. Nat. Hist., (9), 15: 564 (part) - Gulf of Panama.

Chaenophryne longiceps Regan, 1926, Oceanogr. Rep. Danish Dana Exp. 1920-22, 2: 31 (part), pl. 6, fig. 2; Regan and Trewavas, 1932, Dana Rep., 2: 86, fig. 135; Bertelsen, 1951, op. cit., 39: 113, figs. 66, $a, 71$.

Deep-abyssal record and distribution.-Eastern Pacific, 2000 meters, type only.

Length. $-27 \mathrm{~mm}$.

Remarks.-Males and larvae unknown.

\section{Chaenophryne bicornis Regan and Trewavas}

Chaenophryne bicornis Regan and Trewavas, 1932, Dana Rep., 2: 85, fig. 133 -Atlantic, ca. $13^{\circ}$ N., $18^{\circ}$ W., 2500 meters.

Chaenophryne bicornis Bertelsen, 1951, op. cit., 39: 109, 113, fig. 66, $c$.

Deep-abyssal records and distribution.-Eastern Atlantic southeast of the Cape Verde Islands, 2500, 2200 meters, two specimens.

Length. - $18-20 \mathrm{~mm}$.

Remarks.-Males and larvae unknown. 


\section{Chaenophryne quadrifilis Parr}

Chaenophryne longiceps var. quadrifilis Parr, 1927, Bull. Bingham Oceanogr. Coll., 3, (1), p. 22, fig. 8, $a$-off Bermuda.

Chaenophryne longiceps Parr, 1927, op. cit., p. 22, fig. 8, b; Beebe, 1937, Zoologica, 22: 207.

Chaenophryne quadrifilis Regan and Trewavas, 1932, Dana Rep., 2: 87, fig. 136; Parr, 1937, Bull. Bingham Oceanogr. Coll., 3, (7), p. 63; Koefoed, 1944, Rep. Sci. Res. M. Sars No. Atl. Deep-sea Exp. 1910, 4, (2), p. 8, pl. 1, figs. 2, 3; Bertelsen, 1951, Dana Rep., 39: 113.

Deep-abyssal record.-Off Bermuda, 2012 meters, two specimens, including the type.

Distribution.-Eastern Atlantic, ca. $35^{\circ}$ N., $7^{\circ} \mathrm{W}$, one specimen, 1000-1500 meters. Western Atlantic off Bermuda and the Bahamas, two deep-abyssal records and two specimens in 1409 and 1609 meters.

Length. - 25-42 mm.

Remarks.-Males and larvae unknown.

\section{Chaenophryne crenata Regan and Trewavas}

Chaenophryne crenata Regan and Trewavas, 1932, Dana Rep., 2: 86, fig. 134South China Sea, ca. $15^{\circ}$ N., $115^{\circ} \mathrm{E}$.

Chaenophryne crenata Bertelsen, 1951, op. cit., 39: 109, 114, fig. 66, $b$.

Distribution.-Western Pacific, type only, 1000-1500 meters.

Length. $-25 \mathrm{~mm}$.

Remarks.-Males and larvae unknown.

\section{Ghaenophryne crossota Beebe}

Chaenophryne crossotus Beebe, 1932, Zoologica, 13: 83, fig. 21-off Bermuda.

Chaenophryne crossata Regan and Trewavas, 1932, Dana Rep., 2: 86.

Chaenophryne crossota Bertelsen, 1951, op. cit., 39: 114.

Distribution.-Western Atlantic, type only, 914 meters.

Length. $-17 \mathrm{~mm}$.

Remarks.-Males and larvae unknown.

\section{Chaenophryne draco group}

Himantolophus groenlandicus Regan, 1926, Oceanogr. Rep. Danish Dana Exp. 1920-22, 2: 40 (part).

Rhynchoceratias leucorhinus Regan, 1926, op. cit., p. 44 (part).

Trematorhynchus leucorhinus Regan and Trewavas, 1932, Dana Rep., 2: 91 (part). 
Trematorhynchus obliquidens Regan and Trewavas, 1932, op. cit., p. 92, fig. 148.

Chaenophryne draco group Bertelsen, 1951, op. cit., 39: 72, 109, 114, figs. 30, $67,72,73$.

Distribution.-Eastern and western Atlantic. Eastern, southern and western Pacific. North Indian Ocean. South Africa.

Length.-3.5-20.5 mm.

Remarks.--Included under this heading are larvae, males, and metamorphosing females of $C$. draco, $C$. parviconus, and $C$. ramifera.

\section{Chaenophryne draco Beebe}

Chaenophryne draco Beebe, 1932, Zoologica, 13: 84, fig. 22-off Bermuda.

Chaenophryne draco Regan and Trewavas, 1932, Dana Rep., 2: 89; Bertelsen, 1951, op. cit., 39: 116.

Distribution.-Western Atlantic, type only, 1098 meters.

Length.-23 mm.

Remarks.-Males and larvae unknown.

\section{Chaenophryne parviconus Regan and Trewavas}

Chaenophryne parviconus Regan and Trewavas, 1932, Dana Rep., 2: 87, fig. 138-Gulf of Panama, 2000 meters.

Chaenophryne longiceps Regan, 1926, Oceanogr. Rep. Danish Dana Exp. 1920-22, 2: 31 (part).

Chaenophryne haplactis Regan and Trewavas, 1932, Dana Rep., 2: 87, fig. 137.

Chaenophryne atriconus Regan and Trewavas, 1932, op. cit., p. 87, fig. 139.

Chaenophryne columnifera Regan and Trewavas, 1932, op. cit., p. 88, fig. 140.

Chaenophrync melanodactylus Regan and Trewavas, 1932, op. cit., p. 88, fig. 141.

Chaenophryne macractis Regan and Trewavas, 1932, op. cit., p. 89, fig. 142;

Imai, 1942, Jour. Dept. Agr. Kyusyu Imp. Univ., 7: 46, figs. 6, 7.

Chaenophryne melanorhabdus Regan and Trewavas, 1932, Dana Rep., 2: 89, fig. 143.

Chaenophryne pterolophus Regan and Trewavas, 1932, op. cit., p. 89, fig. 144.

Chaenophryne pacis Koefoed, 1944, Rep. Sci. Res. M. Sars No. Atl. Deep-sea Exp. 1910, 4, (2), p. 9, pl. 2, figs. 6, 7.

Chaenophryne parviconus Beebe and Crane, 1947, Zoologica, 31 : 158; Bertelsen, 1951, Dana Rep., 39: 117, fig. 74.

Deep-abyssal records.-Gulf of Panama, 2000-2200 meters, four specimens from three hauls.

Eastern Atlantic, ca. $36^{\circ} \mathrm{N} ., 26^{\circ} \mathrm{W}$. and ca. $30^{\circ} \mathrm{N} ., 20^{\circ} \mathrm{W}$., 2000 meters, two specimens. 
Distribution.-Eastern Atlantic near the Canary Islands and off the Azores, and west to ca. $33^{\circ} \mathrm{W}$., two deep-abyssal records and one specimen in 1500 meters. Eastern Pacific from the Gulf of Panama to ca. $4^{\circ} \mathrm{S}$, $116^{\circ} \mathrm{W}$., three deep-abyssal records (four specimens) and sixteen specimens in 732-1800 meters. Western Pacific, near Japan, one specimen in ca. 1000 meters. North Indian Ocean, ca. $12^{\circ} \mathrm{S} ., 96^{\circ} \mathrm{E}$., one specimen in ca. 1000 meters.

Length. $-14.5-55 \mathrm{~mm}$.

Remarks.-Perhaps a synonym of C. draco (Bertelsen, 1951, p. 118). Males and larvae unknown.

Chaenophryne ramifera Regan and Trewavas

Chaenophryne ramifera Regan and Trewavas, 1932, Dana Rep., 2: 90, fig. 146 - Gulf of Panama.

Chaenophryne fimbriata Regan and Trewavas, 1932, op. cit., p. 90, fig. 145; Belloc, 1938, Rev. Trav. Pêches Marit., 11 : 305, fig. 27.

Chaenophryne intermedia Belloc, 1938, loc. cit., figs. 24, 28.

Chaenophryne ramifera Belloc, 1938, loc. cit., fig. 29; Bertelsen, 1951, Dana Rep., 39: 118, fig. 75 .

Deep-abyssal records.-Eastern Pacific, 2000 meters, the type.

North Indian Ocean, ca. $1^{\circ}$ N., $71^{\circ}$ E., 2000 meters, one specimen.

Distribution.-Eastern Atlantic between the Azores and French West Africa, one specimen in 300-500 meters. Eastern Pacific, one deep-abyssal record. North Indian Ocean, one deep-abyssal record.

Length.-19-24 mm.

Remarks.-Males and larvae unknown.

\section{Lasiognathus saccostoma Regan}

Lasiognathus saccostoma Regan, 1925, Ann. Mag. Nat. Hist., (9), 15: 563Caribbean Sea, ca. $18^{\circ} \mathrm{N} ., 79^{\circ} \mathrm{W}$.

Lasiognathus saccostoma Regan, 1926, Oceanogr. Rep. Danish Dana Exp. 1920-22, 2: 31, pl. 7; Regan and Trewavas, 1932, Dana Rep., 2: 90; Bertelsen, 1951, op. cit., 39: 119, figs. 76, 77, $a$.

Deep-abyssal record and distribution.-Western Atlantic, 2500 meters, type only.

Length. $-75 \mathrm{~mm}$.

Remarks.-Males and larvae unknown.

\section{Lasiognathus beebei Regan and Trewavas}

Lasiognathus beebei Regan and Trewavas, 1932, Dana Rep., 2: 90-off Bermuda. 
Lasiognathus Beebe, 1930, Bull. N. Y. Zool. Soc., 33: 60, figs.; 1932, Nat. Geogr. Mag., 61 : 64, 86, fig.; Gregory, 1933, Trans. Amer. Phil. Soc., 23 : 403 , figs. $275,276, b$.

Lasiognathus beebei Beebe, 1937, Zoologica, 22: 207; Bertelsen, 1951, Dana Rep., 39: 119, fig. 77, $b$.

Distribution.-Western Atlantic, type only, 1098 meters.

Length. $-38 \mathrm{~mm}$.

Remarks.-Males and larvae unknown.

\section{Thaumatichthys pagidostomus Smith and Radcliffe}

Thaumatichthys pagidostomus Smith and Radcliffe, 1912, Proc. U. S. Nat. Mus., 42: 580, pl. 72-Gulf of Tomini, Celebes.

Thaumatichthys pagidostomus Regan, 1926, Oceanogr. Rep. Danish Dana Exp. 1920-22, 2: 31; Regan and Trewavas, 1932, Dana Rep., 2: 91; Bertelsen, 1951, op. cit., 39: 121, figs. 78-80 (misspelled Thaumanichthys).

\section{SYNONYMY OF MALES}

Lipactis tumidus Regan, 1926, Oceanogr. Rep. Danish Dana Exp. 1920-22, 2: 43 (part).

Distribution.-Western Pacific, type only, 1393 meters. Four larvae were taken by the Dana in the western and southeastern Atlantic, and in the Indian Ocean off South Africa. The largest of these, a male approaching metamorphosis, was taken in the Caribbean at a depth of 2000 meters.

Length. $-4.4-84 \mathrm{~mm}$.

\section{Amacrodon binghami Parr}

Thaumatichthys binghami Parr, 1927, Bull. Bingham Oceanogr. Coll., 3, (1), p. 25, fig. 9-off the Bahamas, 1609 meters.

Amacrodon binghami Regan and Trewavas, 1932, Dana Rep., 2: 91; Parr, 1934, Bull. Bingham Oceanogr. Coll., 4, (6), p. 7; Bertelsen, 1951, Dana Rep., 39 : 122.

Distribution.-Western Atlantic off the Bahamas and in the Caribbean Sea, two specimens, 1050-1609 meters.

Length.-?-52 mm.

Remarks.-Males and larvae unknown.

\section{Spiniphryne gladisfenae Beebe}

Dolopichthys gladisfenae Beebe, 1932, Zoologica, 13: 86-off Bermuda.

Centrophryne gladisfenae Regan and Trewavas, 1932, Dana Rep., 2: 84.

Spiniphryne gladisfenae Bertelsen, 1951, op. cit., 39: 122, fig. 81 . 
Bertelsenia gladisfenae Whitley, 1954, Proc. Roy. Zool. Soc. New South Wales, 1952-53: 30.

Distribution.-Western Atlantic, type only, 1280 meters.

Length. $-40 \mathrm{~mm}$.

Remarks.-Males and larvae unknown.

\section{Family CENTROPHRYNIDAE}

This family was introduced by Bertelsen (1951, p. 124) for a single species. The adult male is unknown.

\section{Centrophryne spinulosa Regan and Trewavas}

Centrophryne spinulosa Regan and Trewavas, 1932, Dana Rep., 2: 84, pl. 4, fig. 2 -north of New Guinea, ca. $1^{\circ}$ S., $138^{\circ} \mathrm{E}$.

Centrophryne spinulosa Bertelsen, 1951, op. cit., 39 : 124, 126, figs. 84-87.

\section{SYNONYMY OF MALES}

Rhynchoceratias leucorhinus Regan, 1926, Oceanogr. Rep. Danish Dana Exp. 1920-22, 2: 44 (part).

Deep-abyssal records and distribution.-Western Pacific, 2500 meters, the type.

Eastern Pacific in the Gulf of Panama, 2500, 2000 meters, two specimens.

The Dana took two larvae, one in the Pacific south of the Marquesas Islands and one in the Indian Ocean off South Africa.

Length. $-5.7-51 \mathrm{~mm}$.

\section{Family CERATIIDAE}

The numerous species assigned to this family have been reduced by Bertelsen (1951, p. 127) to two genera, each containing a single widespread species, neither of which belongs to the deep-abyssal fauna. The largest known ceratioid fish is a specimen of Ceratias holboelli $1200 \mathrm{~mm}$. long (Bertelsen, 1951, p. 135). Parasitic males have been found on both species.

\section{Ceratias holboelli Krøyer}

Ceratias holboelli Krøyer, *1844, Naturh. Tidsskr., 1, (2), p. 638 -off Greenland.

Ceratias holboelli Krøyer, *1844, in Gaimard, 1842-56, Voy. Comm. Sci. Nord., 2, pl. 9; Günther, 1861, Cat. Fishes Brit. Mus., 3: 205; 1887, Rep. 
Sci. Res. Voy. Challenger, Zool., 22: 53; Lütken, 1878, Dansk Vidensk. Selsk. Skr., (5), 11, (5), p. 326, figs. 2-8; Goode and Bean, 1895, Ocean. Ichth., p. 489, fig. 399; Jordan and Evermann, 1898, Bull. U. S. Nat. Mus., 47: 2729; 1900, op. cit., fig. 954; Ehrenbaum, 1902, Fauna Arct., 2: 76; *1936, Naturg. Wirtsch. Bedeut. Seefische Nordeur., p. 164, fig. 137; Gill, 1908, Ann. Rep. Smithsonian Inst., p. 578, fig. 12; Saemundsson, 1922, Vidensk. Medd. Dansk naturh. Foren., 74: 163, fig.; 1927, op. cit., 84: 168; 1932, Faune Ichth. Atl. Nord, 10, fig.; 1949, Zool. Iceland, 4, (72), p. 47 (further references, Iceland); Regan, 1925, Naturalist, p. 41, pl. 2; 1925, Proc. Roy. Soc. London, (B), 97: 387, pl. 20, text figs. 1-4; 1926, Oceanogr. Rep. Danish Dana Exp. 1920-22, 2: 34, figs. 1, 20; Beebe, 1926, Bull. N. Y. Zool. Soc., 29: 79, figs.; 1938, op. cit., 41 : 50, figs.; Regan and Trewavas, 1932, Dana Rep., 2: 12, 13, 42, figs. 1, 96; Schnakenbeck, *1936, Der Fischmarkt, (4), 7 : 189, fig.; Rauther, ${ }^{*}$ 1941, Zool. Anz., 136: 131, figs. 1-3; Barbour, 1942, Proc. New England Zool. Club, 21: 81; Bertelsen, 1943, Vidensk. Medd. Dansk naturh. Foren., 107: 185, figs. 1-4; 1951, Dana Rep., 39: 19, 128, 129, 133, pl., text figs. 4, 88, $a, c, 89, b, d, e, 90-92$; Clarke, 1950, Disc. Rep., 26:9, pl. 1, text figs. 1-4; Bigelow and Schroeder, 1953, Fish. Bull., Fish Wildlife Serv., 53, (74), p. 543, fig. 288; Brandes, Kotthaus and Krefft, 1954, Ann. Biol. Cons. Perm. Intern. Expl. Mer, 9: 47 .

Ceratias uranoscopus Murray, in Thomson, *1877, Voy. Challenger, p. 70, fig. 20; Günther, 1887, Rep. Sci. Res. Voy. Challenger, Zool., 22: 54, pl. 11, fig. C.

Mancalias uranoscopus Gill, 1878, Proc. U. S. Nat. Mus., 1: 228; Goode, 1880, op. cit., 3: 469; Goode and Bean, 1895, Ocean. Ichth., p. 490; Jordan and Evermann, 1898, Bull. U. S. Nat. Mus., 47: 2729; Regan, 1926, Oceanogr. Rep. Danish Dana Exp. 1920-22, 2: 37, fig. 21; Parr, 1927, Bull. Bingham Oceanogr. Coll., 3, (1), p. 30; 1932, Bull. Boston Soc. Nat. Hist., 63: 12, fig. 4; Norman, 1930, Disc. Rep., 2: 355; 1939, Sci. Rep. John Murray Exp. 1933-34, 7, (1), p. 116; Regan and Trewavas, 1932, Dana Rep., 2: 13, 99, fig. 158; Beebe, 1937, Zoologica, 22: 207; Barbour, 1942, Proc. New England Zool. Club, 21: 77, pl. 9; Imai, 1942, Jour. Dept. Agr. Kyusyu Imp. Univ., 7: 43, figs. 4, 5; Beebe and Crane, 1947, Zoologica, 31 : 169 (misspelled Mancalius).

Typhlopsaras shufeldti Gill, 1883, Forest and Stream, p. 284; Barbour, 1942, Proc. New England Zool. Club, 21 : 78, pl. 9.

Ceratias shufeldti Günther, 1887, Rep. Sci. Res. Voy. Challenger, Zool., 22: 54 .

Mancalias shufeldti Goode and Bean, 1895, Ocean. Ichth., p. 490, fig. 401; Jordan and Evermann, 1898, Bull. U. S. Nat. Mus., 47: 2730; 1900, op. cit., fig. 955; Gill, 1908, Ann. Rep. Smithsonian Inst., p. 579, fig. 14.

Miopsaras myops Gilbert, 1905, Bull. U. S. Fish Comm., 1903: 695, pl. 99; Fowler, 1928, Mem. Bishop Mus., 10: 475.

Ceratias n. sp. Murray and Hjort, 1912, Depths of Ocean, pp. 81, 82, fig. 59. Mancalias tentaculatus Norman, 1930, Disc. Rep., 2: 355, fig. 45; Regan and Trewavas, 1932, Dana Rep., 2: 100.

Mancalias xenistius Regan and Trewavas, 1932, op. cit., p. 99, pl. 6, fig. 2. 
Mancalias bifilis Regan and Trewavas, 1932, op. cit., p. 100, pl. 6, fig. 1.

Mancalias uranoscopus triflos Roule and Angel, 1933, Rés. Camp. Sci. Monaco, 86: 57, pl. 3, fig. 27; Belloc, 1949, Bull. Inst. Océanogr. Monaco, 958: 17. Mancalias sessilis Imai, 1941, Jap. Jour. Zool., 9: 245, figs. 12, 13; 1942 , Jour. Dept. Agr. Kyusyu Imp. Univ., 7: 45.

Typhloceratias firthi Barbour, 1942, Proc. New England Zool. Club, 21: 78, pls. 10,11 , fig. 2 .

Parrichthys merrimani Barbour, 1942, op. cit., p. 84, pl. 11, fig. 1.

Ceratias holboelli xenistius Bertelsen, 1943, Vidensk. Medd. Dansk Naturh. Foren., 107: 203.

Ceratias holboelli sessilis Bertelsen, 1943, op. cit., p. 203.

Ceratias tentaculatus Bertelsen, 1943, op. cit., p. 203.

Reganichthys giganteus Bigelow and Barbour, 1944, Proc. New England Zool. Club, 23: 9, pls. 4-6.

Reganula gigantea Bigelow and Barbour, 1944, Copeia, p. 123.

Mancalias kroyeri Koefoed, 1944, Rep. Sci. Res. M. Sars No. Atl. Deep-sea Exp. 1910, 4, (2), p. 11, pl. 3, fig. 4.

Deep-abyssal records.-Eastern Atlantic, ca. $22^{\circ}$ N., $22^{\circ}$ W., 4390 meters, one specimen.

Western Atlantic, ca. $40^{\circ} \mathrm{N}$., $56^{\circ} \mathrm{W} ., 4000$ meters, one specimen.

Western Atlantic, $17^{\circ}-25^{\circ} \mathrm{N} ., 60^{\circ}-76^{\circ}$ W., 2000-3000 meters, four specimens in three hauls.

Arabian Sea, 2091 meters, one specimen.

Indian Ocean, ca. $5^{\circ}$ N., $80^{\circ}-90^{\circ}$ E., 2200,2000 meters, two specimens.

Western Pacific, ca. $5^{\circ}$ S., $130^{\circ}$ E., 2000 meters, one specimen.

Distribution.- North Atlantic off Iceland and Greenland; from $38^{\circ} \mathrm{N}$. to $4^{\circ} \mathrm{N}$. in the east; and in the west from off Nova Scotia to Bermuda, the Caribbean, and off South America in ca. $7^{\circ} \mathrm{N} ., 46^{\circ}$ W. South Atlantic, ca. $7^{\circ} \mathrm{S} ., 8^{\circ} \mathrm{W}$. and ca. $52^{\circ} \mathrm{S} ., 9^{\circ} \mathrm{E}$. Indian Ocean off South Africa. North Indian Ocean from the Arabian Sea to ca. $9^{\circ} \mathrm{S} ., 114^{\circ} \mathrm{E}$. (off Java). Western Pacific off Japan, in the South China Sea and around the Dutch East Indies. Mid-Pacific off Hawaii. South Pacific off New Zealand and near New Caledonia. Eastern Pacific near Cocos Island, the Galapagos Islands, and in ca. $4^{\circ} \mathrm{S} ., 116^{\circ} \mathrm{W}$. Antarctic, ca. $61^{\circ} \mathrm{S} ., 102^{\circ} \mathrm{E}$.

There are about sixty-five specimens known, of which only ten have been reported from deep-abyssal waters. Large adult examples have all been found in relatively shallow water, some of them from an unknown depth, others in 120-680 meters. The only known Antarctic specimen was taken from the stomach of a sperm whale. 
Smaller examples were caught between 200 and 1700 meters, one of them from a closing net hauled in 650-700 meters in the south Atlantic (ca. $52^{\circ}$ S., $9^{\circ}$ E.).

Larvae are known from the eastern, western, and southeastern Atlantic, the southern and western Pacific, and the north Indian Ocean.

Length.-3-1200 mm.

Remarks.-Free-living males were first described by Bertelsen (1951, p. 137), who also listed the seven known males parasitic on adult females whose length ranged between 850 and $1130 \mathrm{~mm}$.

\section{Cryptopsaras couesi Gill}

Cryptopsaras couesii Gill, 1883, Forest and Stream, p. 284-Atlantic off coast of United States, ca. $38^{\circ}$ N., $68^{\circ}$ W., 3084 meters.

Cryptopsaras (Cryptosparas of authors) couesii Günther, 1887, Rep. Sci. Res. Voy. Challenger, Zool., 22: 55; Goode and Bean, 1895, Ocean. Ichth., p. 491, fig. 402; Jordan and Evermann, 1898, Bull. U. S. Nat. Mus., 47: 2731; 1900, op. cit., fig. 956; Gill, 1908, Ann. Rep. Smithsonian Inst., p. 579, fig. 13; Roule, 1916, Bull. Inst. Océanogr. Monaco, 320: 27; 1919, Rés. Camp. Sci. Monaco, 52: 76, pl. 5, fig. 6; Regan, 1925, Proc. Roy. Soc. London, (B), 97 : 393, fig. 5; 1926, Oceanogr. Rep. Danish Dana Exp. 1920-22, 2: 35, pl. 9, fig. 2; Barnard, 1927, Ann. So. Afr. Mus., 21 : 1005; Parr, 1927, Bull. Bingham Oceanogr. Coll., 3, (1), p. 29; 1930, Copeia, p. 135, fig. 8; Norman, 1930, Disc. Rep., 2 : 354, fig. 44 ; Regan and Trewavas, 1932, Dana Rep., 2: 12, 96, fig. 154; Oshima, 1934, Rec. Oceanogr. Works Japan, 6: 108, fig.; Beebe, 1937, Zoologica, 22: 207; Kuronuma, 1941, Bull. Biogeogr. Soc. Japan, 11: 64; Barbour, 1941, Proc. New England Zool. Club, 19: 11, pl. 4, fig. 2, pl. 6, figs. 5, 6; Imai, 1942, Jour. Dept. Agr. Kyusyu Imp. Univ., 7: 42, fig. 3; Koefoed, 1944, Rep. Sci. Res. M. Sars No. Atl. Deep-sea Exp. 1910, 4, (2), p. 11; Smith, 1949, Sea Fishes So. Afr., p. 428, fig. 1229; Bertelsen, 1951, Dana Rep., 39: 14, 17, 22, 23, $128,129,139,145$, figs. $2,3,6, b, 7,88, b, 89, a, c, 93-97$; Abe and $\mathrm{Na}$ kamura, 1954, Jap. Jour. Ichth., 3: 95; Grey, 1955, Fieldiana, Zool., 37 : 299.

Ceratias carunculatus Günther, 1887, Rep. Sci. Res. Voy. Challenger, Zool., 22: 55, pl. 11, fig. D.

Cryptopsaras carunculatus Goode and Bean, 1895, Ocean. Ichth., p. 491; Regan, 1926, Oceanogr. Rep. Danish Dana Exp. 1920-22, 2: 36; Regan and Trewavas, 1932, Dana Rep., 2: 98, fig. 155; Norman, 1939, Sci. Rep. John Murray Exp. 1933-34, 7, (1), p. 115; Phillipps, 1941, Trans. Roy. Soc. New Zealand, 71: 160, pl. 27; Barbour, 1941, Proc. New England Zool. Club, 19: 11, pl. 3, fig. 2, pl. 6, figs. 7, 8 .

Ceratias couesii Brauer, 1906, Wiss. Ergebn. Deutschen Tiefsee Exp. Valdivia, 15, (1), p. 317 , pl. 15, fig. 7; 1908, op. cit., 15: (2), p. 184, pl. 32, fig. 17, pl. 34, fig. 17; Murray and Hjort, 1912, Depths of Ocean, pp. 92, 608, 614, 627, fig. 466; Saemundsson, 1922, Vidensk. Medd. Dansk naturh. 
Foren., 74: 160, pl. 3; 1932, Faune Ichth. Atl. Nord, 10, fig.; 1949, Zool. Iceland, 4, (72), p. 47 (further references, Iceland); Borodin, 1931, Bull. Mus. Comp. Zool., 72: 83; Fowler, 1936, Bull. Amer. Mus. Nat. Hist., 70: 1137 , fig. 477.

Ceratias (Paraceratias) mitsukurii Tanaka, 1908, Jour. Coll. Sci. Imp. Univ. Tokyo, 23, (13), p. 18, pl. 2, fig. 3.

Paraceratias mitsukurii Tanaka, 1911, Fig. Descr. Fishes Japan, 2: 30, pl. 8, fig. 25; Jordan, Tanaka, and Snyder, 1913, Jour. Coll. Sci. Imp. Univ. Tokyo, 33, (1), p. 427; Barbour, 1941, Copeia, p. 175.

Ceratias sp. Regan, 1916, Rep. Brit. Antarctic (Terra Nova) Exp. 1910, Zool., 1, (4), p. 147, pl. 10, fig. 1.

Cryptopsaras mitsukurii Regan, 1926, Oceanogr. Rep. Danish Dana Exp. 1920-22, 2: 36; Regan and Trewavas, 1932, Dana Rep., 2: 98; Barbour, 1941, Proc. New England Zool. Club, 19: 7, 10, pl. 5.

Melanocetus sp. Beebe, 1926, Arcturus Adv., figs. 50, 53.

Cryptopsaras valdiviae Regan and Trewavas, 1932, Dana Rep., 2: 98, fig. 156. Cryptopsaras pennifer Regan and Trewavas, 1932, op. cit., p. 98, fig. 157.

Cryptopsaras normani Regan and Trewavas, 1932, op. cit., p. 98; Barbour, 1941, Proc. New England Zool. Club, 19: 11, pl. 4, fig. 1, pl. 6, figs. 1, 2; Imai, 1942, Jour. Dept. Agr. Kyusyu Imp. Univ., 7: 39, figs. 1, 2; Beebe and Crane, 1947, Zoologica, 31 : 168, fig. 14.

Cryptosparas Gregory, 1933, Trans. Amer. Phil. Soc., 23: 401, fig. 273.

Cryptosparas atlantidis Barbour, 1941, Proc. New England Zool. Club, 19: 11, pl. 3, pl. 6, figs. 3, 4 .

Ceratias holboelli Koefoed, 1944, Rep. Sci. Res. M. Sars No. Atl. Deep-sea Exp. 4, (2), p. 10, pl. 2, fig. 2.

Cryptosaras couesii Fowler, 1949, Mem. Bishop Mus., 12: 160.

Deep-abyssal records.-Eastern Atlantic, ca. $36^{\circ}$ N., $26^{\circ}$.W., 3500 meters, one specimen.

Eastern Atlantic, ca. $39^{\circ}$ N., $18^{\circ}$ W. and ca. $21^{\circ}$ N., $22^{\circ}$ W., 2100, 2000 meters, two specimens.

Mid-Atlantic, ca. $28^{\circ}$ N., $56^{\circ}$ W., 4000 meters, one specimen.

Mid-Atlantic, ca. $28^{\circ} \mathrm{N}$., $56^{\circ} \mathrm{W}$. and ca. $31^{\circ} \mathrm{N}$., $41^{\circ} \mathrm{W}$., 2000 2500 meters, five specimens from two stations (three nets).

Western Atlantic, 3084 meters, the type.

Western Atlantic, $17^{\circ}-34^{\circ}$ N., $60^{\circ}-70^{\circ}$ W., $2000-2500$ meters, five specimens from four hauls.

South Atlantic, ca. $23^{\circ}$ S., $3^{\circ}$ E. and ca. $35^{\circ}$ S., $18^{\circ}$ E., 2000 2500 meters, three specimens.

North Indian Ocean, ca. $1^{\circ}$ N., $71^{\circ}$ E. and ca. $5^{\circ}$ N., $80^{\circ}$ E., 2300-2500 meters, two specimens.

Arabian Sea and Gulf of Aden, 2091, 1840 meters, three specimens. 
Western Pacific, $4^{\circ}-5^{\circ}$ S., $128^{\circ}-130^{\circ}$ E., $2000-2500$ meters, two specimens.

Southwestern Pacific, ca. $33^{\circ}$ S., $154^{\circ}-165^{\circ}$ E., 2000 meters, two specimens from two hauls.

South Pacific, ca. $14^{\circ}$ and $18^{\circ}$ S., $147^{\circ}-163^{\circ}$ E., $2000-2500$ meters, three specimens from three hauls.

Eastern Pacific, ca. $0^{\circ} 18^{\prime}$ S., $99^{\circ} \mathrm{W}$. and ca. $4^{\circ}$ S., $116^{\circ} \mathrm{W}$, 2000 meters, three specimens.

Distribution.-Atlantic, Iceland (one specimen) and from ca. $50^{\circ}$ $\mathrm{N}$. to ca. $7^{\circ} \mathrm{N} ., 46^{\circ} \mathrm{W}$. on the western side, and in the east, south to Cape Point, South Africa. Indian Ocean off South Africa. North Indian Ocean from the Gulf of Aden and the Arabian Sea south to ca. $7^{\circ}$ S., $41^{\circ}$ E. and east to ca. $9^{\circ}$ S., $114^{\circ}$ E. Western Pacific off Japan and around the Philippines and the Dutch East Indies. Southwestern Pacific off Australia and New Zealand $\left(33^{\circ}-35^{\circ} \mathrm{S}\right.$.) and in Cook Strait, New Zealand (ca. $42^{\circ}$ S.). South Pacific near the Society Islands and Samoa. Eastern Pacific from the Gulf of Panama to ca. $4^{\circ} \mathrm{S} ., 116^{\circ} \mathrm{W}$. Larvae known from all areas inhabited by older examples.

Of about 150 known specimens only twenty-eight have been recorded from deep-abyssal waters, the rest from the surface to 1700 meters.

Length.-2.5-440 mm.

Remarks.-Two parasitic males recorded. Free-living males first described by Bertelsen (1951, p. 144).

\section{Family GIGANTACTINIDAE}

Bertelsen (1951, pp. 145-154, figs. 98-103) described the first known larvae belonging to the family Gigantactinidae. Those of the genus Gigantactis can be separated into two types but none can definitely be assigned to any of the described species, which are based on adult or adolescent female specimens. Gigantactis larvae were taken by the Dana in the north Atlantic, the western, southern and eastern Pacific, and the Indian Ocean. Bertelsen has noted that Beebe and Rose (1926, p. 49) figured a male Gigantactis larva.

Bertelsen also identified the first gigantactinid males. Those of the genus Gigantactis include Teleotrema Regan and Trewavas (see G. microphthalmus, below), Teleotrema sp. Nybelin (1948, p. 89) from the northwestern Atlantic, and five Dana specimens, one from the eastern Atlantic in 2500 meters and four from the Caribbean 
Sea and western Atlantic in 1500-2000 meters. Like the larvae, males cannot be assigned to the described species, the taxonomy of which is most uncertain.

Also unassigned to any species is a metamorphosing female Gigantactis taken at a depth of 2500 meters in the Indian Ocean (Bertelsen, 1951, p. 150, fig. 99, d).

\section{Gigantactis macronema Regan}

Gigantactis macronema Regan, 1925, Ann. Mag. Nat. Hist., (9), 15: 565Atlantic, ca. $31^{\circ} \mathrm{N} ., 41^{\circ} \mathrm{W}$.

Gigantactis macronema Regan, 1926, Oceanogr. Rep. Danish Dana Exp. 1920-22, 2: 38, pl. 11; Regan and Trewavas, 1932, Dana Rep., 2: 94; Bertelsen, 1951, op. cit., 39: 150, fig. 101.

Deep-abyssal record and distribution.-Mid-Atlantic, 3500 meters, type only.

Length. - $133 \mathrm{~mm}$.

\section{Gigantactis gracilicauda Regan}

Gigantactis gracilicauda Regan, 1925, Ann. Mag. Nat. Hist., (9), 15: 565Caribbean Sea, ca. $13^{\circ}$ N., $61^{\circ} \mathrm{W}$.

Gigantactis gracilicauda Regan, 1926, Oceanogr. Rep. Danish Dana Exp. 1920-22, 2: 38, pl. 10, fig. 2; Regan and Trewavas, 1932, Dana Rep., 2: 94; Bertelsen, 1951, op. cit., 39: 150 .

Deep-abyssal record and distribution.-Western Atlantic, 2700 meters, type only.

Length. $-107 \mathrm{~mm}$.

\section{Gigantactis sexfilis Regan and Trewavas}

Gigantactis sexfilis Regan and Trewavas, 1932, Dana Rep., 2: 94, pl. 5, fig. 2, text fig. 150 -Atlantic, ca. $13^{\circ} \mathrm{N}$, $57^{\circ} \mathrm{W}$.

Gigantactis vanhoeffeni Regan, 1926, Oceanogr. Rep. Danish Dana Exp. 1920-22, 2: 38 (part).

Gigantactis sexfilis Bertelsen, 1951, Dana Rep., 39: 150.

Deep-abyssal record and distribution.-Western Atlantic, 2500 meters, type only.

Length. $-70 \mathrm{~mm}$.

\section{Gigantactis vanhoeffeni Brauer}

Gigantactis vanhoeffeni Brauer, 1902, Zool. Anz., 25: 296-Indian Ocean, ca. $5^{\circ} \mathrm{S} ., 43^{\circ} \mathrm{E}$.

Gigantactis vanhoeffeni Brauer, 1906, Wiss. Ergebn. Deutschen Tiefsee Exp. Valdivia, 15, (1), p. 322, pl. 15, figs. 8, 9; 1908, op. cit., 15, (2), pp. 103, 184, pl. 31, figs. 18-20, pl. 32 , figs. $1-5$, pl. 34 , fig. 14, pl. 44, fig. 1; Gill, 
1908, Ann. Rep. Smithsonian Inst., p. 586, fig. 25; Regan and Trewavas, 1932, Dana Rep., 2: 93, 94; Fowler, 1936, Bull. Amer. Mus. Nat. Hist., 70 : 1345, fig. 565; Bertelsen, 1951, Dana Rep., 39: 150.

Deep-abyssal records and distribution.-North Indian Ocean east of Zanzibar, 2500 meters, the type.

North Indian Ocean, ca. $6^{\circ}$ S., $73^{\circ}$ E., 1900 meters, one specimen.

Length. - 30-35 mm.

Remarks.-A $30 \mathrm{~mm}$. specimen from the Atlantic (ca. $25^{\circ} \mathrm{N}$., $20^{\circ}$ W., 3500 meters), listed as this species by Regan (1926, p. 38), was said by Bertelsen (1951, p. 150) to be a distinct species.

\section{Gigantactis exodon Regan and Trewavas}

Gigantactis exodon Regan and Trewavas, 1932, Dana Rep., 2: 94, pl. 5, fig. 1, text fig. 151 -Caribbean Sea, ca. $18^{\circ} \mathrm{N}$., $79^{\circ} \mathrm{W}$.

Gigantactis vanhoeffeni Regan, 1926, Oceanogr. Rep. Danish Dana Exp. 1920-22, 2: 38 (part).

Gigantactis exodon Bertelsen, 1951, Dana Rep., 39: 150.

Distribution.-Western Atlantic, type only, 1700 meters.

Length. $-32 \mathrm{~mm}$.

\section{Gigantactis perlatus Beebe and Crane}

Gigantactis perlatus Beebe and Crane, 1947, Zoologica, 31 : 167, pl. 2, fig. 3, text fig. $13-$ Pacific off Panama, ca. $7^{\circ}$ N., $81^{\circ} \mathrm{W}$.

Gigantactis perlatus Bertelsen, 1951, Dana Rep., 39: 150.

Distribution.-Eastern Pacific, type only, 914 meters.

Length. $-42 \mathrm{~mm}$.

\section{Gigantactis ovifer Regan and Trewavas}

Gigantactis ovifer Regan and Trewavas, 1932, Dana Rep., 2: 95, fig. 152South China Sea, ca. $14^{\circ}$ N., $119^{\circ} \mathrm{E}$.

Gigantactis ovifer Bertelsen, 1951, op. cit., 39: 150 .

Distribution.-Western Pacific, type only, 1700 meters.

Length. $-39 \mathrm{~mm}$.

\section{Gigantactis filibulbosus Fraser-Brunner}

Gigantactis filibulbosus Fraser-Brunner, 1935, Proc. Roy. Irish Acad., B42: 326-Atlantic, ca. $53^{\circ} \mathrm{N} ., 12^{\circ} \mathrm{W}$.

Gigantactis filibulbosus Bertelsen, 1951, Dana Rep., 39: 150.

Distribution.-Eastern Atlantic off southwestern Ireland, type only, 320 meters.

Length. $-25 \mathrm{~mm}$. (without caudal). 


\section{Gigantactis longicirra Waterman}

Gigantactis longicirra Waterman, 1939, Bull. Mus. Comp. Zool., 85: 82, figs. 1, 2-Atlantic, ca. $39^{\circ} \mathrm{N} ., 70^{\circ} \mathrm{W}$.

Gigantactis longicirra Waterman, 1948, Jour. Morph., 82: 81, figs. 1-10; Bertelsen, 1951, Dana Rep., 39: 150.

Distribution.-Western Atlantic off the coast of the United States, type only, 1000 meters, taken in a closing net.

Length. $-62 \mathrm{~mm}$.

Gigantactis microphthalmus Regan and Trewavas

Teleotrema microphthalmus Regan and Trewavas, 1932, Dana Rep., 2: 93, fig. 149 -Atlantic, ca. $8^{\circ} \mathrm{N} ., 15^{\circ} \mathrm{W}$.

Gigantactis microphthalmus Bertelsen, 1951, op. cit., 39: 152, figs. 102, b, $103, d-e$.

Deep-abyssal records and distribution.--Eastern Atlantic, 3500 meters, the type.

Western Atlantic, ca. $17^{\circ} \mathrm{N} ., 64^{\circ} \mathrm{W} ., 2500$ meters, one specimen.

Length.-20.5-22.5 mm.

Remarks.-Both specimens are males.

\section{Rhynchactis leptonema Regan}

Rhynchactis leptonema Regan, 1925, Ann. Mag. Nat. Hist., (9), 15: 565Atlantic, ca. $8^{\circ} \mathrm{N} ., 44^{\circ} \mathrm{W}$.

Rhynchactis leptonema Regan, 1926, Oceanogr. Rep. Danish Dana Exp. 1920-22, 2: 38, pl. 10, fig. 1; Regan and Trewavas, 1932, Dana Rep., 2: 95; Bertelsen, 1951, op. cit., 39: 154, fig. 104.

Deep-abyssal records and distribution.-Western Atlantic, 2000 meters, the type, a female.

South Pacific near Samoa, ca. $7^{\circ}$ S., $167^{\circ}$ W., 2500 meters, one male specimen.

Larvae were taken by the Dana in the western Atlantic and north Indian Oceans.

Length. $-3-57 \mathrm{~mm}$.

\section{Family NEOCERATIIDAE}

The family contains only one species, of which the male is known to be parasitic.

\section{Neoceratias spinifer Pappenheim}

Neoceratias spinifer Pappenheim, 1914, Deutsche Südpolar-Exp. 1901-1903, 15, Zool., 7: 198, fig. 10-Atlantic near St. Helena, ca. $12^{\circ} \mathrm{S} ., 6^{\circ} \mathrm{W}$. 
Neoceratias spinifer Regan, 1926, Oceanogr. Rep. Danish Dana Exp. 1920-22,

2: 39, fig. 22; Regan and Trewavas, 1932, Dana Rep., 2: 95, fig. 153; Fowler, 1936, Bull. Amer. Mus. Nat. Hist., 70: 1146, fig. 484; Koefoed, 1944, Rep. Sci. Res. M. Sars No. Atl. Deep-sea Exp. 1910, 4, (2), p. 9, pl. 2, fig. 5; Bertelsen, 1951, Dana Rep., 39: 158, figs. 105-107.

Deep-abyssal records. - Southeastern Atlantic, 2000 meters, the type.

Western Pacific, ca. $3^{\circ}$ N., $137^{\circ}$ E., 2500 meters, a female specimen with a parasitic male.

Distribution.-Mid- and southeastern Atlantic, one deep-abyssal record and one specimen in 1700 meters (ca. $34^{\circ} \mathrm{N} ., 33^{\circ} \mathrm{W}$.). Western Pacific, one deep-abyssal record.

The Dana took larvae in the north Atlantic, the north Indian Ocean and the western Pacific.

Length. $-4-60 \mathrm{~mm}$.

\section{Family LINOPHRYNIDAE}

Bertelsen (1951) divided linophrynids into five genera and about nineteen species. He was unable to separate larvae of Edriolychnus schmidti from those of Linophryne arborifera and for all of these larvae he retained the name of "Hyaloceratias." Parasitic males have been found in four species and probably occur throughout the family.

\section{Photocorynus spiniceps Regan}

Photocorynus spiniceps Regan, 1925, Proc. Roy. Soc. London, (B), 97: 393, figs. 6, 7-Gulf of Panama, 1700 meters.

Photocorynus spiniceps Regan, 1926, Oceanogr. Rep. Danish Dana Exp. 1920-22, 2: 21, pl. 1, fig. 1, text fig. 2; Regan and Trewavas, 1932, Dana Rep., 2: 15, 102; Bertelsen, 1951, op. cit., 39: 163, 165, figs. 108, a, 109, 110.

Deep-abyssal records.-Gulf of Panama, 2200-2500 meters, four specimens from three hauls.

Distribution.-Eastern Pacific in the Gulf of Panama, four specimens from deep-abyssal hauls and the type, a female with a parasitic male, from 1700 meters.

Length.-11.5-62 mm.

Remarks.-Larvae unknown.

Edriolychnus schmidti Regan. Table 29.

Edriolychnus schmidti Regan, 1925, Proc. Roy. Soc. London, (B), 97: 398, figs. 8, 9-Caribbean Sea, ca. $13^{\circ}$ N., $61^{\circ}$ W., 2700 meters. 
Edriolychnus schmidti Regan, 1926, Oceanogr. Rep. Danish Dana Exp. 192022, 2: 25, pl. 3, fig. 2, text fig. 5; Roule and Angel, 1930, Rés. Camp. Sci. Monaco, 79: 122, pl. 6, figs. 156-158; Regan and Trewavas, 1932, Dana Rep., 2: 15, 104, pl. 9, fig. 2, text figs. 3-5; Fowler, 1936, Bull. Amer. Mus. Nat. Hist., 70: 1342, fig. 562; Grey, 1955, Fieldiana, Zool., 37: 299.

Edriolychnus sp. Beebe, 1930, Bull. N. Y. Zool. Soc., 33: 205, 242, figs.; 1937, Zoologica, 22: 207; Regan and Trewavas, 1932, Dana Rep., 2: 104, fig. 163.

Edriolychnus macracanthus Regan and Trewavas, 1932, op. cit., p. 104.

Edriolychnus radians Regan and Trewavas, 1932, op. cit., pp. 17, 104, pl. 9, fig. 1, text fig. 64 .

Edriolychnus roulei Regan and Trewavas, 1932, op. cit., p. 105.

Edriolychnus schmidti (?= Haplophryne mollis) Bertelsen, 1951, op. cit., 39: 163, 168, figs. $108, b, 111, a, b, 112,113$.

\section{SYNONYMY OF MALES}

?Aceratias mollis Brauer, 1902, Zool. Anz., 25: 297; 1906, Wiss. Ergebn. Deutschen Tiefsee Exp. Valdivia, 15, (1), p. 324, pl. 16, fig. 10.

? Haplophryne mollis Regan, 1912, Ann. Mag. Nat. Hist., (8), 9: 289; 1926, Oceanogr. Rep. Danish Dana Exp. 1920-22, 2: 25 (part).

Aceratias macrorhinus indicus juvenes Murray and Hjort, 1912, Depths of Ocean, p. 745, fig. 536; Koefoed, 1944, Rep. Sci. Res. M. Sars No. Atl. Deep-sea Exp. 1910, 4, (2), p. 15, pl. 2, figs. 3, 4.

Haplophryne sp. (?Edriolychnus male) Regan and Trewavas, 1932, Dana Rep., 2: 111 (part); ?Beebe, 1937, Zoologica, 22: 207 (part).

Distribution.-North Atlantic from ca. $35^{\circ} \mathrm{N}$. to ca. $8^{\circ} \mathrm{N}$., $44^{\circ} \mathrm{W}$. in the west and to ca. $25^{\circ} \mathrm{N} ., 20^{\circ} \mathrm{W}$. in the east. South Atlantic, ca. $23^{\circ} \mathrm{S}$., $3^{\circ} \mathrm{E}$. Eastern Pacific, Gulf of Panama. Southwestern Pacific around New Caledonia, east of the Kermadec Islands (ca. $30^{\circ} \mathrm{S} ., 176^{\circ} \mathrm{W}$.) and near Lord Howe Island. Indian Ocean off Sumatra (ca. $6^{\circ} \mathrm{S} ., 105^{\circ}$ E.) and in ca. $26^{\circ}$ S., $93^{\circ} \mathrm{E}$.

The species is probably chiefly deep-abyssal. Of twenty-seven recorded specimens (thirty-two, including the five known parasitic males) only seven were caught above 2000 meters. All females with parasitic males have been taken at or below a depth of about 2000 meters. Extreme depth range 200-4000 meters.

Larvae are recorded as "Hyaloceratias" (p. 278).

Length.-17-63 mm.

\section{Linophryne algibarbata Waterman}

Linophryne algibarbata Waterman, 1939, Bull. Mus. Comp. Zool., 85: 85, figs. 3 , 4-Atlantic, ca. $39^{\circ}$ N., $70^{\circ}$ W., 400 meters. 
Linophryne algibarbata Koefoed, 1944, Rep. Sci. Res. M. Sars No. Atl. Deepsea Exp. 1910, 4, (2), p. 13, pl. 2, fig. 1; Bertelsen, 1951, Dana Rep., 39: 175.

\section{SYNONYMY OF MALES}

Aceratias macrorhinus Koefoed, 1944, Rep. Sci. Res. M. Sars No. Atl. Deepsea Exp. 1910, 4, (2), p. 15 (part).

Distribution.-Western Atlantic only, the type in a closing net in 400 meters and two specimens in ca. $40^{\circ} \mathrm{N}$., $50^{\circ} \mathrm{W}$., $400-500$ and 1100-1500 meters, respectively.

Length. - 24-48 mm.

Remarks.-Larvae unknown.

\section{Linophryne lucifer Collett}

Linophryne lucifer Collett, 1886, Proc. Zool. Soc. London, p. 138, pl. 15Atlantic, ca. $36^{\circ}$ N., $20^{\circ} \mathrm{W}$.

Linophryne lucifer Günther, 1887, Rep. Sci. Res. Voy. Challenger, Zool., 22 : 57 ; Goode and Bean, 1895, Ocean Ichth., p. 496, fig. 408; Gill, 1908, Ann. Rep. Smithsonian Inst., p. 584, fig. 23; Regan, 1926, Oceanogr. Rep. Danish Dana Exp. 1920-22, 2: 24; Regan and Trewavas, 1932, Dana Rep., 2: 106, 109 (spelled lucifera); Fowler, 1936, Bull. Amer. Mus. Nat. Hist., 70: 1141, fig. 480; Bertelsen, 1951, Dana Rep., 39: 175, 176.

Distribution.-Eastern Atlantic off Madeira, the type only, found alive and helpless at the surface with a fish larger than itself in its stomach.

Length. $-49 \mathrm{~mm}$.

Remarks.-Males and larvae unknown.

\section{Linophryne coronata Parr}

Linophryne coronata Parr, 1927, Bull. Bingham Oceanogr. Coll., 3, (1), p. 13, fig. 4-Atlantic off the Bahama Islands, 1609 meters.

Linophryne longibarbata Borodin, 1930, Proc. New England Zool. Club, 11 : 87; 1931, Bull. Mus. Comp. Zool., 72: 83; Regan and Trewavas, 1932, Dana Rep., 2: 107.

Linophryne coronata Regan and Trewavas, 1932, op. cit., p. 110; Parr, 1934, Bull. Bingham Oceanogr. Coll., 4, (6), pp. 44, 49, figs. 15, 16; Bertelsen, 1951, Dana Rep., 39: 175, 176.

Linophryne coronata longibarbata Parr, 1934, Bull. Bingham Oceanogr. Coll., 4, (6), pp. 45, 52, figs. 17, 18.

Linophryne coronata diphlegma Parr, 1934, op. cit., pp. 7, 45, 49, 54, figs. 19, 20.

Distribution.-Western Atlantic only, ca. $25^{\circ}$ N., $77^{\circ} \mathrm{W}$. to ca. $37^{\circ} \mathrm{N} ., 67^{\circ} \mathrm{W}$., four specimens, $1050-1609$ meters. 
Length.-33-75 mm. (without caudal).

Remarks.-Males and larvae unknown; L. masculina (p. 277) may be the male.

\section{Linophryne quinqueramosus Beebe and Crane}

Linophryne quinqueramosus Beebe and Crane, 1947, Zoologica, 31: 174, pl. 3, fig. 5, text fig. 17-Gulf of Panama.

Linophryne quinqueramosus Bertelsen, 1951, Dana Rep., 39: 176.

Distribution.-Eastern Pacific, type only, 914 meters.

Length. $-89 \mathrm{~mm}$.

Remarks.-Males and larvae unknown.

\section{Linophryne corymbifera Regan and Trewavas}

Linophryne corymbifera Regan and Trewavas, 1932, Dana Rep., 2: 110, pl. 10, fig. 1, text figs. 69, 70-Celebes Sea.

Linophryne corymbifera Bertelsen, 1951, op. cit., 39: 177, fig. 116.

\section{SYNONYMY OF MALES}

Aceratias indicus Regan and Trewavas, 1932, Dana Rep., 2: 113 (part).

Deep-abyssal records.-Banda Sea, ca. $5^{\circ} \mathrm{S} ., 130^{\circ}$ E., 2500 meters, two young male specimens.

North Indian Ocean, ca. $1^{\circ}$ N., $71^{\circ}$ E., 2200 meters, one young male.

Distribution.-North Indian Ocean and western Pacific, three males from deep-abyssal waters and the type, a female, in 300-500 meters. Larvae known from the same areas.

Length. $-10-48 \mathrm{~mm}$.

Remarks.-Younger larvae of this species may be included in "Hyaloceratias" (p. 278).

Linophryne arborifera Regan. Table 30.

Linophryne arborifer Regan, 1925, Ann. Mag. Nat. Hist., (9), 15: 564Atlantic, ca. $14^{\circ} \mathrm{N}$., $28^{\circ} \mathrm{W} ., 200-300$ meters.

Linophryne arborifer Regan, 1926, Oceanogr. Rep. Danish Dana Exp. 1920-22, 2: 24, pl. 3, fig. 1; Parr, 1927, Bull. Bingham Oceanogr. Coll., 3, (1), p. 10, fig. 3; Beebe, 1932, Zoologica, 13: 90, fig. 24.

Haplophryne mollis Regan, 1926, Oceanogr. Rep. Danish Dana Exp. 1920-22, 2: 25 (part), pl. 3, fig. 3.

Linophryne brevibarbata Beebe, 1932, Zoologica, 13: 94, figs. 26, 27; 1937, op. cit., 22: 207; Regan and Trewavas, 1932, Dana Rep., 2: 109; Parr, 1934, Bull. Bingham Oceanogr. Coll., 4, (6), p. 43. 
Cryptolychnus micractis Regan and Trewavas, 1932, Dana Rep., 2: 45, 105.

Linophryne arborifera Regan and Trewavas, 1932, op. cit., pp. 105, 111;

Beebe, 1932, Nat. Geogr. Mag., 61: 85; 1937, Zoologica, 22: 207; Parr,

1934, Bull. Bingham Oceanogr. Coll., 4, (6), p. 44; Bertelsen, 1951, Dana Rep., 39: 21, 168, 178, figs. 5, 111, $c-d, 117,118$; Grey, 1955, Fieldiana, Zool., $37: 300$.

Linophryne eupogon Regan and Trewavas, 1932, Dana Rep., 2: 110.

Linophryne arborifera eupogon Parr, 1934, Bull. Bingham Oceanogr. Coll., 4, (6), pp. 7, 44, 46, fig. 14 .

Linophryne densiramus Imai, 1941, Jap. Jour. Zool., 9: 247, figs. 14-17; 1942, Jour. Dept. Agr. Kyusyu Imp. Univ., $7: 48$.

\section{SYNONYMY OF MALES}

Aceratias macrorhinus indicus Brauer, 1902, Zool. Anz., 25: 297; 1906, Wiss. Ergebn. Deutschen Tiefsee Exp. Valdivia, 15, (1), p. 325, pl. 16, figs. 6-9; Murray and Hjort, 1912, Depths of Ocean, pp. 87, 90, 96, 609, 615, 627, figs. 68, 470; Koefoed, 1944, Rep. Sci. Res. M. Sars No. Atl. Deep-sea Exp. 1910, 4, (2), p. 13 (part).

Aceratias indicus Gill, 1908, Ann. Rep. Smithsonian Inst., p. 587, figs. 27, 28; Regan, 1912, Ann. Mag. Nat. Hist., (8), 9: 289; 1926, Oceanogr. Rep. Danish Dana Exp. 1920-22, 2: 45, fig. 26; Regan and Trewavas, 1932, Dana Rep., 2 : 113 (part).

Lipactis tumidus Regan, 1926, Oceanogr. Rep. Danish Dana Exp. 1920-22, 2: 43 (part).

Rhynchoceratias onchorhynchus Regan, 1926, op. cit., p. 44 (part).

? Haplophryne simus Borodin, 1930, Occ. Pap. Boston Nat. Hist. Soc., 5: 285; 1931, Bull. Mus. Comp. Zool., 72: 83, pl. 4, fig. 1; Regan and Trewavas, 1932, Dana Rep., 2: 112.

Nannoceratias denticulatus Regan and Trewavas, 1932, op. cit., p. 112, fig. 172.

Deep-abyssal records.-Eastern Atlantic, ca. $25^{\circ} \mathrm{N} ., 20^{\circ} \mathrm{W}$, 4000 meters, one male specimen.

Eastern Atlantic, ca. $33^{\circ} \mathrm{N} ., 16^{\circ} \mathrm{W}$. and ca. $7^{\circ} \mathrm{S} ., 8^{\circ} \mathrm{W} ., 3500$ meters, two male specimens.

Eastern Atlantic, ca. $29^{\circ}$ N., $25^{\circ} \mathrm{W}$. and ca. $36^{\circ}$ N., $29^{\circ}$ W., 2000 meters, two male specimens.

Mid-Atlantic, ca. $31^{\circ}$ N., $35^{\circ}$ W., 2500 meters, one male specimen.

Mid-Atlantic, ca. $28^{\circ} \mathrm{N}$., $56^{\circ} \mathrm{W} ., 2000$ meters, two male specimens.

Western Atlantic, ca. $25^{\circ}$ N., $76^{\circ}$ W., 2000 meters, one male specimen.

Southwestern Pacific, ca. $33^{\circ}$ S., $154^{\circ}-165^{\circ}$ E., $2200-2500$ meters, three male specimens from three nets. 
Western Pacific, ca. $4^{\circ}$ S., $128^{\circ}$ E., 2500 meters, one male specimen.

North Indian Ocean, ca. $6^{\circ}$ S., $73^{\circ}$ E., 1900 meters, two male specimens.

Distribution.-Eastern Atlantic from south of the Azores (ca. $36^{\circ}$ N., $29^{\circ} \mathrm{W}$.) to ea. $7^{\circ} \mathrm{S} ., 8^{\circ} \mathrm{W}$., five deep-abyssal specimens, two in 1000-1500 meters and two in 100-200 meters. Mid-Atlantic, three deep-abyssal specimens and one, ca. $34^{\circ} \mathrm{N}$., $47^{\circ} \mathrm{W}$., in 1700 meters. Western Atlantic from ca. $54^{\circ}$ to ca. $25^{\circ} \mathrm{N}$., one deep-abyssal record and twelve specimens in 500-1645 meters. Southwestern Pacific between Australia and New Zealand, deep-abyssal records only. Western Pacific in the Banda Sea (a deep-abyssal record), and off Japan, one specimen in 200-400 meters. North Indian Ocean near Chagos Archipelago and the Seychelles Islands, one deep-abyssal record and one specimen in 1500 meters. Males perhaps deepabyssal. Deepest capture of a female, 1645 meters.

Length. - 25-70 mm.

Remarks.-Larvae are included in "Hyaloceratias" (p. 278). Parasitic males have not yet been found.

\section{Linophryne arcturi Beebe}

Diabolidium arcturi Beebe, 1926, Bull. N. Y. Zool. Soc., 29: 80, fig.-Pacific, ca. $4^{\circ} \mathrm{N} ., 87^{\circ} \mathrm{W}$.

Diabolidium arcturi Beebe, 1926, Arcturus Adv., fig. 1.

Linophryne arcturi Regan and Trewavas, 1932, Dana Rep., 2 : 107; Parr, 1934, Bull. Bingham Oceanogr. Coll., 4, (6), p. 42; Beebe and Crane, 1947, Zoologica, 31 : 173, fig. 16; Bertelsen, 1951, Dana Rep., 39: 183.

Distribution.--Eastern Pacific south of Cocos Island, type only, 914 meters.

Length. $-38.6 \mathrm{~mm}$.

Remarks.-Males and larvae unknown.

\section{Linophryne bicornis Parr}

Linophryne bicornis Parr, 1927, Bull. Bingham Oceanogr. Coll., 3, (1), p. 9, fig. 2-Atlantic off Bermuda, 1609 meters.

Linophryne bicornis Regan and Trewavas, 1932, Dana Rep., 2: 111; Parr, 1934, Bull. Bingham Oceanogr. Coll., 4, (6), p. 43; Bertelsen, 1951, Dana Rep., 39: 175, 184; Grey, 1955, Fieldiana, Zool., 37: 300, fig. 56.

Distribution.-Western Atlantic off Bermuda, two specimens, 1000-1609 meters. 
Length. $-27 \mathrm{~mm}$. (without caudal) and $42 \mathrm{~mm}$.

Remarks.-Males and larvae unknown.

\section{Linophryne polypogon Regan}

Linophryne polypogon Regan, 1925, Ann. Mag. Nat. Hist., (9), 15: 565Atlantic, ca. $32^{\circ} \mathrm{N} ., 16^{\circ} \mathrm{W}$.

Linophryne polypogon Regan, 1926, Oceanogr. Rep. Danish Dana Exp. 192022, 2: 24, pl. 2, fig. 3; Regan and Trewavas, 1932, Dana Rep., 2: 108; Parr, 1934, Bull. Bingham Oceanogr. Coll., 4, (6), p. 43; Bertelsen, 1951, Dana Rep., 39: 183, figs. 120, $a, 121, a$.

Deep-abyssal record and distribution.-Eastern Atlantic, 3500 meters, type only.

Length. $-45 \mathrm{~mm}$.

Remarks.-Males and larvae probably included in the $L$. macrorhinus group (p. 277).

\section{Linophryne macrodon Regan}

Linophryne macrodon Regan, 1925, Ann. Mag. Nat. Hist., (9), 15: 564-Gulf of Panama.

Linophryne macrodon Regan, 1926, Oceanogr. Rep. Danish Dana Exp. 1920 22, 2: 24, pl. 2, fig. 2; Regan and Trewavas, 1932, Dana Rep., 2: 107; Parr, 1934, Bull. Bingham Oceanogr. Coll., 4, (6), p. 43; Bertelsen, 1951, Dana Rep., 39: 185, figs. 120, $b, 121, c$.

Deep-abyssal record and distribution.-Eastern Pacific, 2200 meters, type only.

Length. $-53 \mathrm{~mm}$.

Remarks.-Males and larvae probably included in the $L$. macrorhinus group (p. 277).

\section{Linophryne brevibarbis Parr}

Linophryne brevibarbis Parr, 1927, Bull. Bingham Oceanogr. Coll., 3, (1), p. 7, fig. 1-Atlantic off Bermuda.

Linophryne brevibarbis Regan and Trewavas, 1932, Dana Rep., 2: 108; Parr, 1934, Bull. Bingham Oceanogr. Coll., 4, (6), p. 43; Bertelsen, 1951, Dana Rep., 39: 185.

Deep-abyssal record and distribution.-Western Atlantic, 2012 meters, type only.

Length. $-34 \mathrm{~mm}$.

Remarks.-Males and larvae probably included in the $L$. macrorhinus group (p. 277). 
Linophryne racemifera Regan and Trewavas

Linophryne racemifera Regan and Trewavas, 1932, Dana Rep., 2: 108, pl. 10, fig. 2 , text figs. 165,166 -Atlantic, ca. $24^{\circ} \mathrm{N}$., $17^{\circ} \mathrm{W}$.

Linophryne racemifera Parr, 1934, Bull. Bingham Oceanogr. Coll., 4, (6), p. 43;

Bertelsen, 1951, Dana Rep., 39: 185, fig. 121, $b$.

Deep-abyssal record and distribution.-Eastern Atlantic, 2500 meters, type only.

Length. $-70 \mathrm{~mm}$.

Remarks.-Males and larvae probably included in the L. macrorhinus group (see below).

\section{Linophryne argyresca Regan and Trewavas}

Linophryne argyresca Regan and Trewavas, 1932, Dana Rep., 2: 19, 108, pl. 10, fig. 3 , text figs. $9,167,168$-Indian Ocean, ca. $5^{\circ}$ N., $90^{\circ}$ E.

Linophryne argyresca Parr, '1934, Bull. Bingham Oceanogr. Coll., 4, (6), p. 44; Bertelsen, 1951, Dana Rep., 39: 185, fig. 121, $e$.

Deep-abyssal record and distribution.--North Indian Ocean, 2200 meters, a female with a parasitic male.

Length. $-84 \mathrm{~mm}$.

Remarks.-Larvae and free-living males perhaps included in the L. macrorhinus group (see below).

\section{Linophryne masculina Parr}

Borophryne masculina Parr, 1934, Bull. Bingham Oceanogr. Coll., 4, (6), pp.

7, 56, fig. 21-off the Bahama Islands.

Borophryne masculina Beebe and Crane, 1947, Zoologica, 31 : 176.

Linophryne masculina Bertelsen, 1951, Dana Rep., 39: 186.

Distribution.-Western Atlantic, one male specimen, 1050-1100 meters.

Length. $-24 \mathrm{~mm}$.

Remarks.-Perhaps the male of $L$. coronata.

\section{Linophryne macrorhinus group}

Aceratias macrorhinus Brauer, 1902, Zool. Anz., 25: 296-Atlantic, ca. $9^{\circ}$ S., $9^{\circ}$ E., 2000 meters.

Aceratias macrorhinus Brauer, 1906, Wiss. Ergebn. Deutschen Tiefsee Exp. Valdivia, 15, (1), p. 324, pl. 16, figs. 4, 5; 1908, op. cit., 15, (2), p. 184, fig.; Gill, 1908, Ann. Rep. Smithsonian Inst., p. 587, fig. 26; Regan and Trewavas, 1932, Dana Rep., 2: 113; Koefoed, 1944, Rep. Sci. Res. M. Sars No. Atl. Deep-sea Exp. 1910, 4, (2), p. 15 (part), pl. 3, fig. 5. 
Haplophryne hudsonius Beebe, 1929, Zoologica, 12: 19; 1929, op. cit., p. 23, figs. 2-5; Parr, 1930, Occ. Pap. Bingham Oceanogr. Coll., 3: 14; Gregory, 1933, Trans. Amer. Phil. Soc., 23: 407, fig. 280.

Haplophryne mollis Norman, 1930, Disc. Rep., 2 : 352.

Cryptolychnus paucidens Regan and Trewavas, 1932, Dana Rep., 2: 105, pl. 8, fig. 2.

Anomalophryne hudsonius Regan and Trewavas, 1932, op. cit., p. 112.

Aceratias edentula Beebe, 1932, Zoologica, 13: 102, fig. 31; 1937, op. cit., 22: 207.

Linophryne macrorhinus group Bertelsen, 1951, Dana Rep., 39: 184, 186, figs. $121, d, 122$.

Distribution.-Eastern, western and southeastern Atlantic. Eastern, southern and western Pacific. North Indian Ocean.

Length.--3.5-30 $\mathrm{mm}$.

Remarks.--In this group Bertelsen (1951) placed about fifty male and larval specimens not at present identified with any described species of Linophryne. The following species are perhaps represented in the group: polypogon, macrodon, brevibarbis, racemifera, argyresca(?).

\section{"Hyaloceratias"}

Haplophryne mollis Regan, 1916, Brit. Antarctic (Terra Nova) Exp. 1910, Zool., 1 : 148, pl. 10, fig. 2.

Haplophryne sp. Regan and Trewavas, 1932, Dana Rep., 2: 111 (part), fig. 171.

Haplophryne triregium Whitley and Phillipps, 1939, Trans. Roy. Soc. New Zealand, 69: 236.

Hyaloceratias parri Koefoed, 1944, Rep. Sci. Res. M. Sars No. Atl. Deep-sea Exp. 1910, 4, (2), p. 16, pl. 1, figs. 7, 8.

"Hyaloceratias" Bertelsen, 1951, Dana Rep., 39: 168, 172, 189, figs. 123, 124.

Distribution.-North Atlantic. Eastern Pacific. South Pacific off New Zealand. Western Pacific. North Indian Ocean.

Length. $-6-23 \mathrm{~mm}$.

Remarks.-Under this name Bertelsen (1951) grouped 235 larval specimens belonging to Edriolychnus schmidti, Linophryne arborifera and possibly L. corymbifera.

\section{Acentrophryne longidens Regan}

Acentrophryne longidens Regan, 1926, Oceanogr. Rep. Danish Dana Exp. 1920-22, 2: 23, pl. 1, fig. 2-Gulf of Panama, 1700 meters.

Acentrophryne longidens Regan and Trewavas, 1932, Dana Rep., 2: 106; Beebe and Crane, 1947, Zoologica, 31 : 170, fig. 15; Nigrelli, 1947, op. cit., p. 183, pls. 1-4, figs. 2-10; Bertelsen, 1951, Dana Rep., 39: 192. 
Distribution.-Eastern Pacific in the Gulf of Panama and off Costa Rica, two specimens, 1700 and 914 meters.

Length. $-60-75 \mathrm{~mm}$.

Remarks.-Males and larvae unknown.

Borophryne apogon Regan. Table 31.

Borophryne apogon Regan, 1925, Ann. Mag. Nat. Hist., (9), 15: 564-Gulf of Panama, 2200 meters.

Borophryne apogon Regan, 1926, Oceanogr. Rep. Danish Dana Exp. 1920-22, 2: 23, pl. 2, fig. 1; Regan and Trewavas, 1932, Dana Rep., 2: 12, 18, 106, figs. 7, 8; Beebe and Crane, 1947, Zoologica, 31 :171, pl. 2, fig. 4 Bertelsen, 1951, Dana Rep., 39: 163, 193, figs. 108, $d, 125,126$.

\section{SYNONYMY OF MALES}

Aceratias macrorhinus Regan, 1926, Oceanogr. Rep. Danish Dana Exp. 1920-22, 2: 45, fig. 27.

Distribution.-Eastern Pacific, Gulf of Panama and south of Cocos Island. Forty specimens known, including six parasitic males. Perhaps partially deep-abyssal in habitat. Four male larvae recorded from the Gulf of Panama. Extreme depth range 200-2700 meters.

Length. $-7-83 \mathrm{~mm}$.

\section{Allector cheloniae Heller and Snodgrass}

Allector cheloniae Heller and Snodgrass, 1903, Proc. Washington Acad. Sci., 5: 228, fig. 1-Galapagos Islands.

Allector cheloniae Regan, 1926, Oceanogr. Rep. Danish Dana Exp. 1920-22, 2: 25; Parr, 1930, Occ. Pap. Bingham Oceanogr. Coll., 3: 14; Regan and Trewavas, 1932, Dana Rep., 2: 103; Fowler, 1936, Bull. Amer. Mus. Nat. Hist., 70: 1368; Böhlke, 1953, Stanf. Ichth. Bull., 5: 148.

Distribution.--Eastern Pacific, one damaged specimen of uncertain taxonomic position, taken from the stomach of a green turtle.

Length. $-27 \mathrm{~mm}$. 


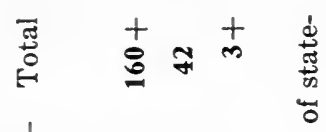

衰总

嵞

鰝 |

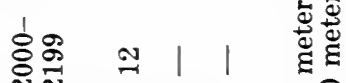

\%ู้ ลง -

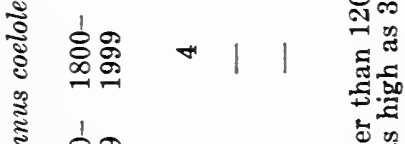

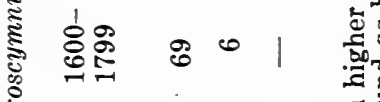

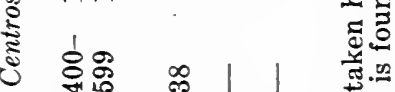

话兽

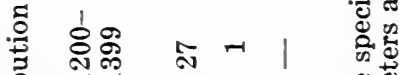

黄

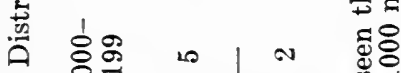

可

营 do

ᄀ 옹

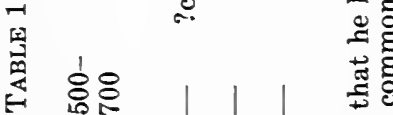

言

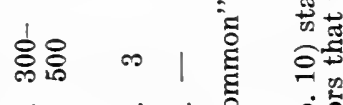

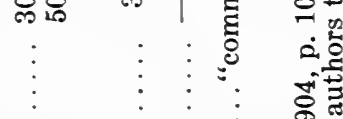

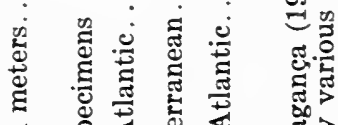

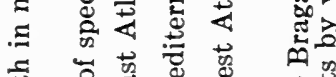

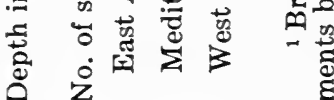

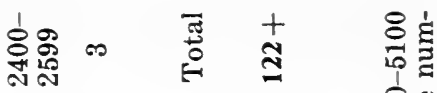

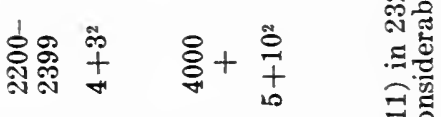

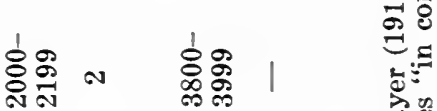

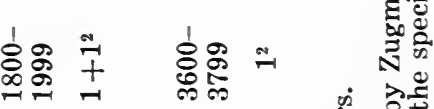

$\therefore$

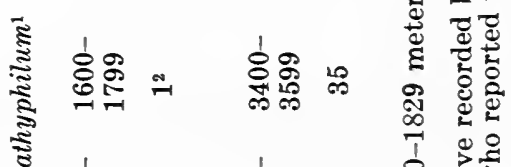

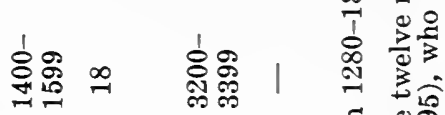

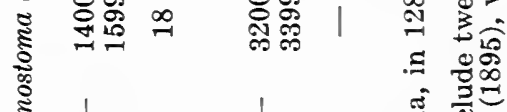

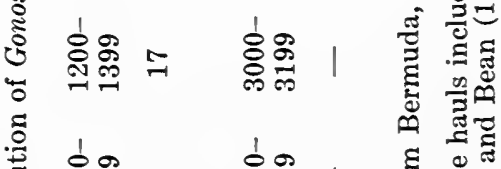

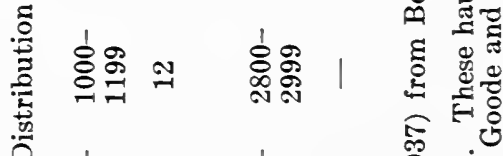

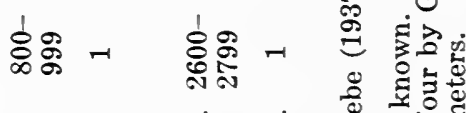

密名

so

政

동

원

os

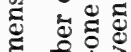

है

के 2 \%

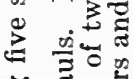

里可

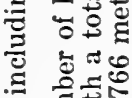

政

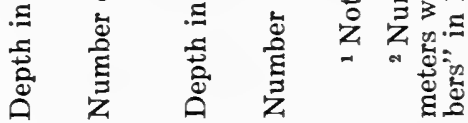




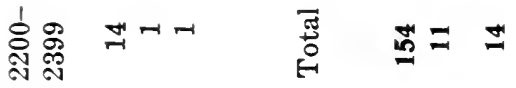

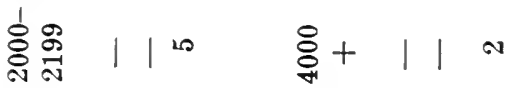

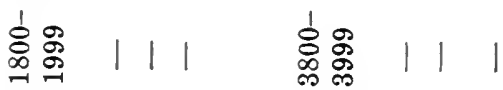

高总 | | | 高总 | | |

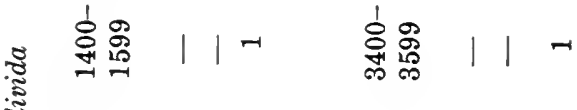

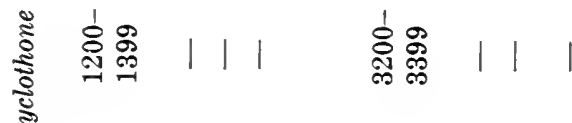

రิ

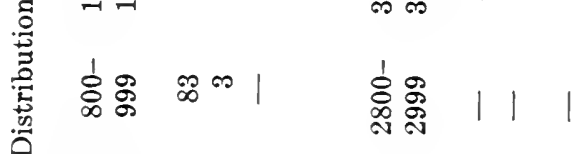

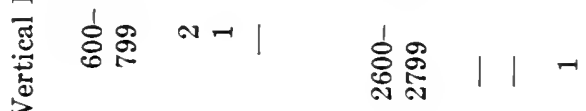

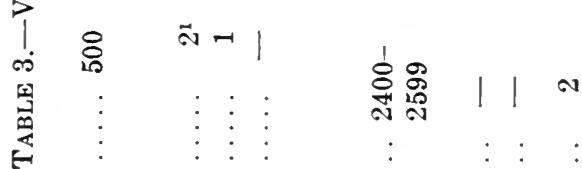




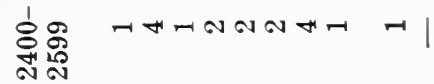

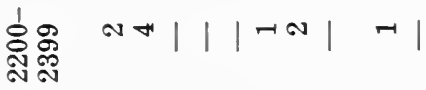

各需

ஓ्ठ요묨

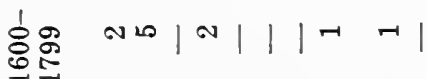

过露

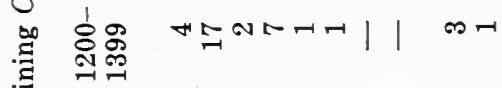

రి임

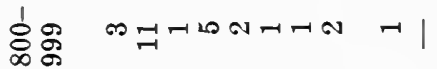

¿

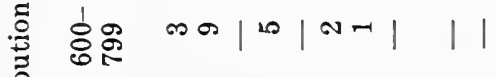

营

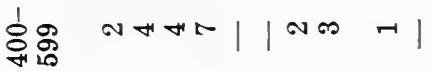

유요 $N$ - | | | | |

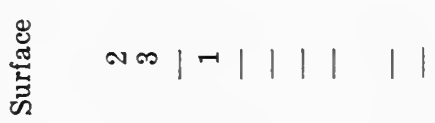

क

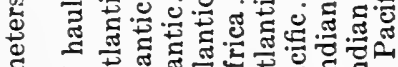

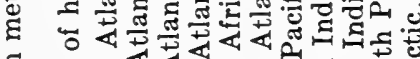

.

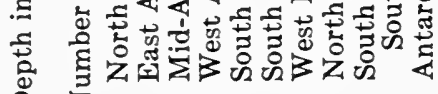

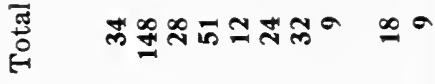

$8+1|-1| 1|1| 1$

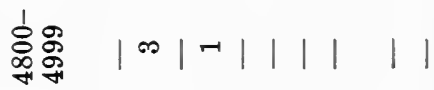

迡尔 |Nー||-1| | |

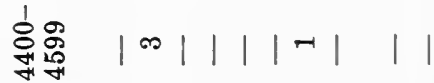

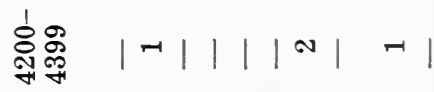

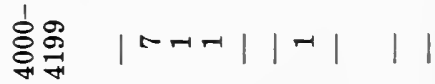

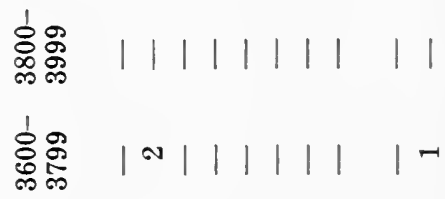

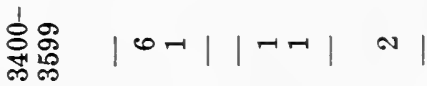

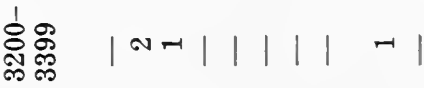

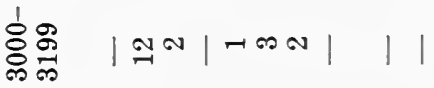

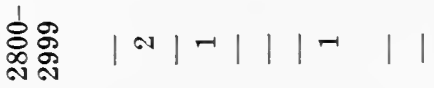

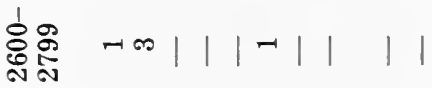

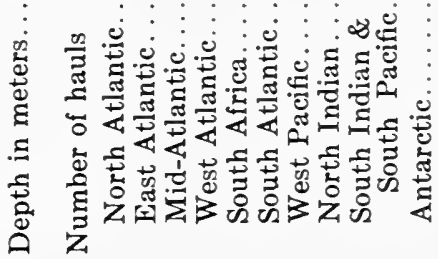




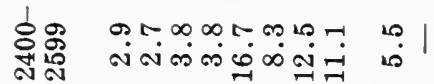

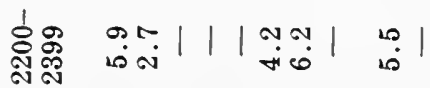

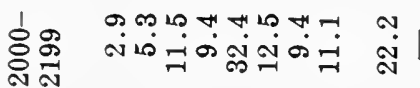

胥

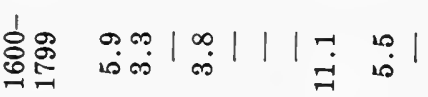

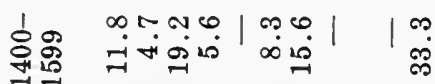

రిळ

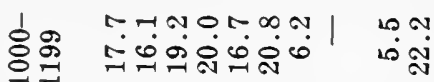

de

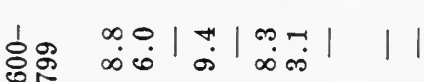

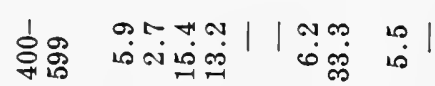

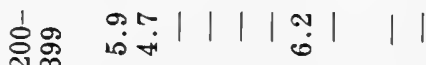

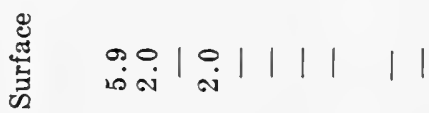

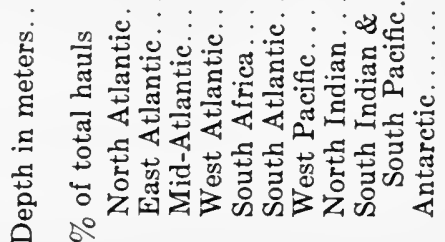

$\oint_{0}^{\circ}+|| \begin{aligned} & \infty \\ & \infty\end{aligned}|||| \mid$

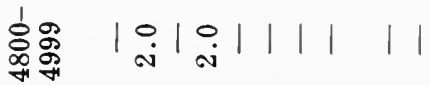

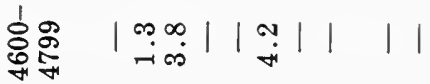

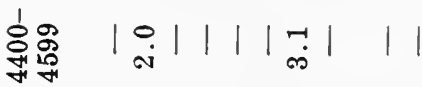

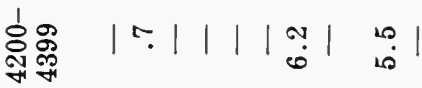

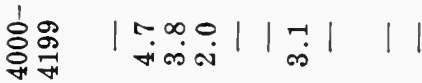

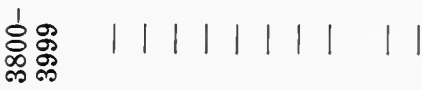

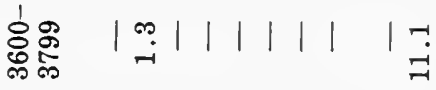

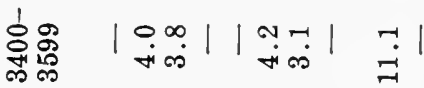

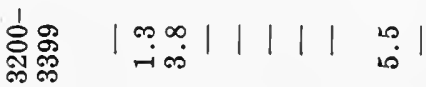

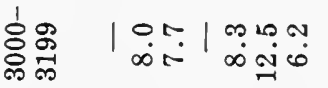

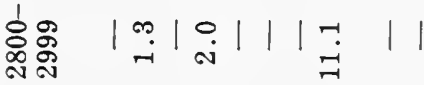

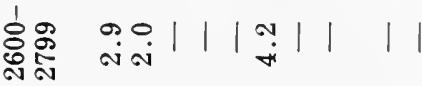

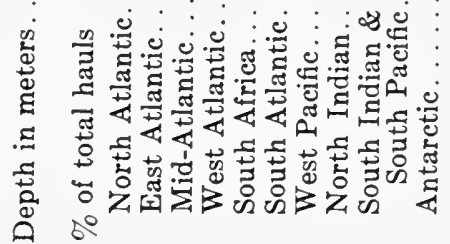




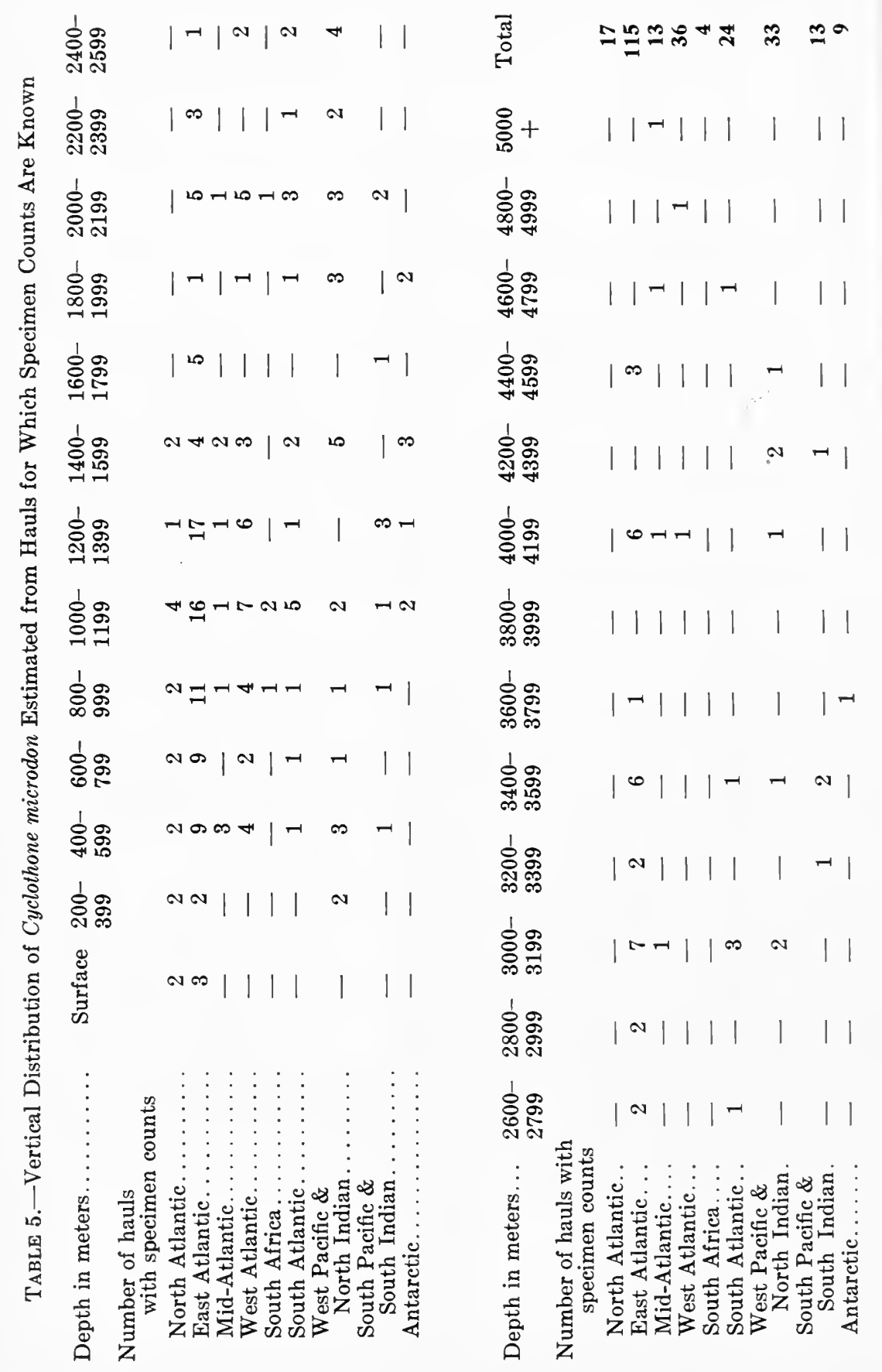




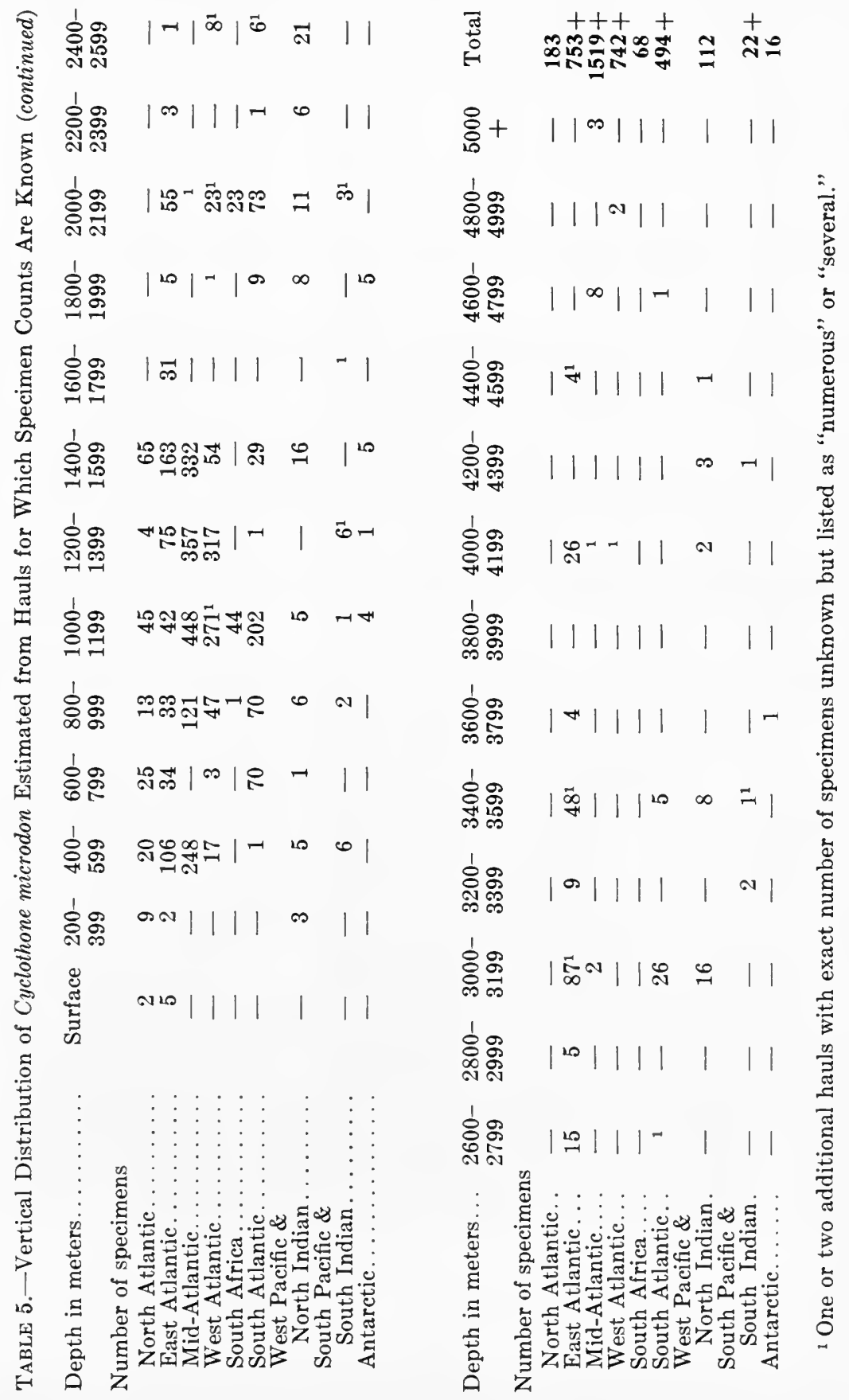




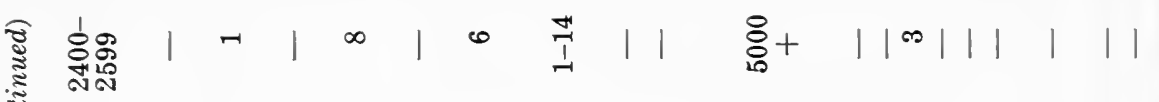

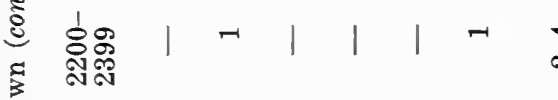

स11 密兽

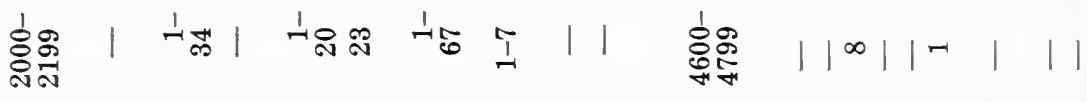

ร कूล

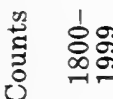

密密密 1 th

$110 \pm 15$

せ 䇏

क

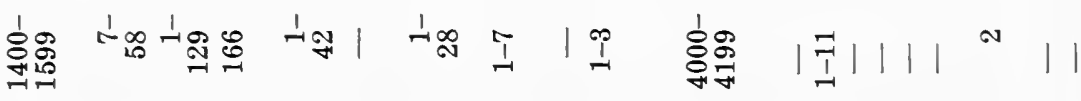

递

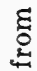

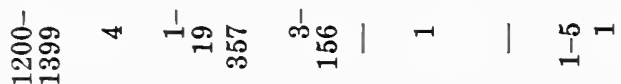

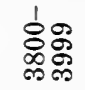

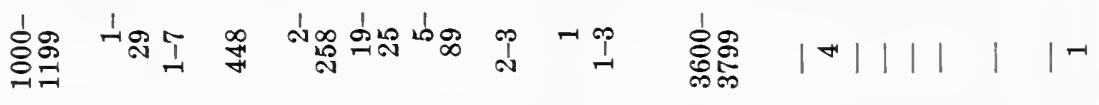

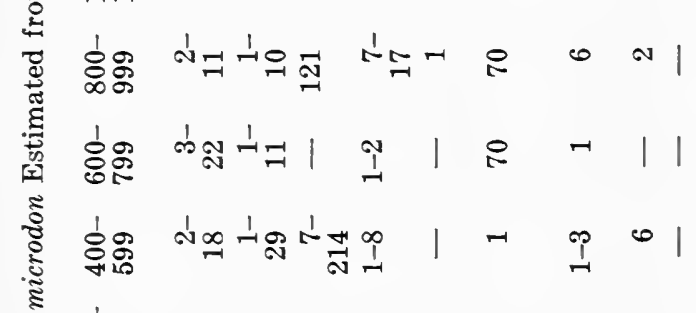

竎 离总

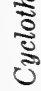

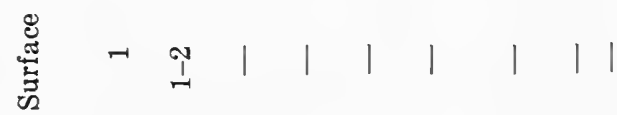

.

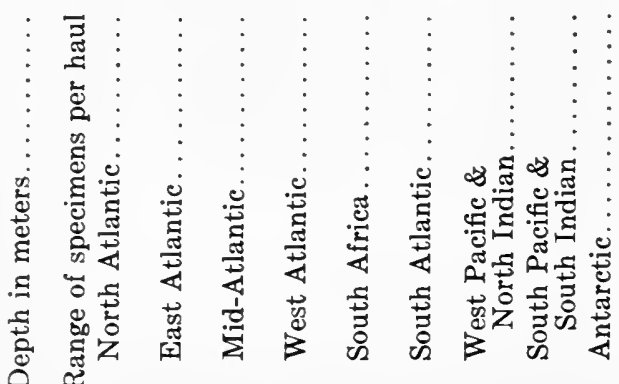

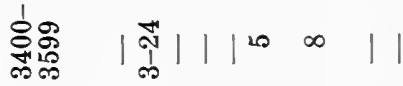

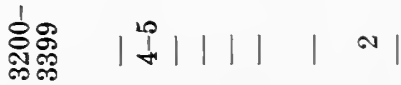

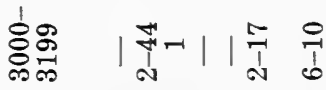

高恋 |

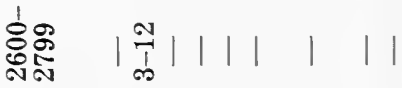

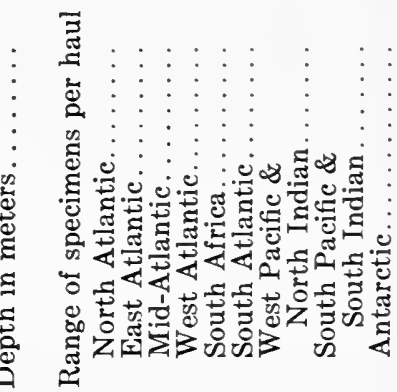


ళ్

은

ई

잉

4

晃

๕ี

号

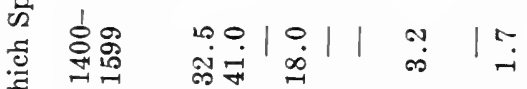

运

总

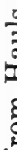

ฐ

总 8

ठํㅇ

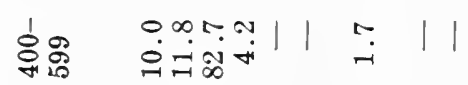

ठ্ণু

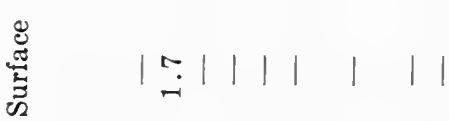

을

:

i. 峁

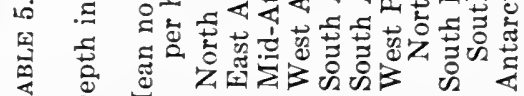

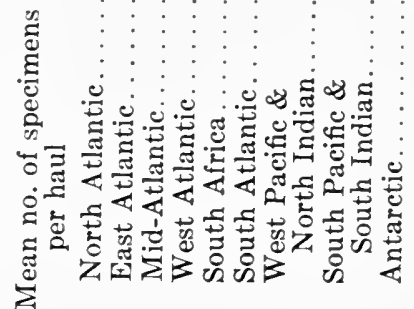

8+

产。

实究

악 용

过房

ஓे

용

유요

하요

$\left|\stackrel{\infty}{\infty}^{0}\right|||||$

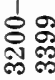

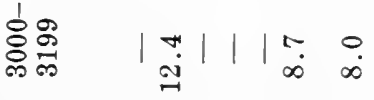

¿े.

옹ㅇำ

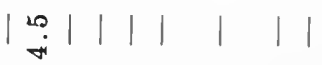

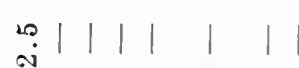

疍

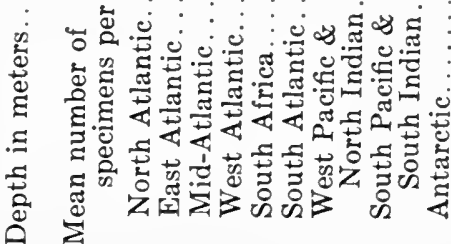




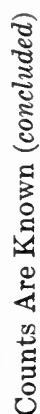

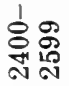

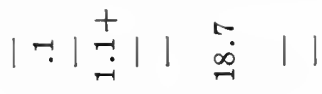

s.

มูฐ

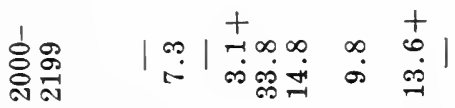

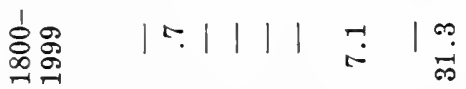

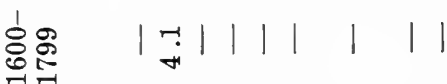

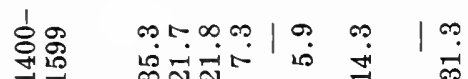

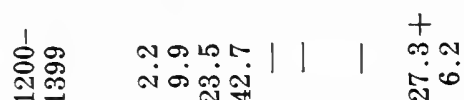

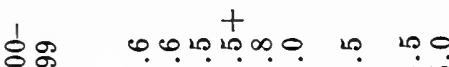

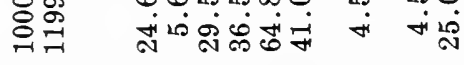

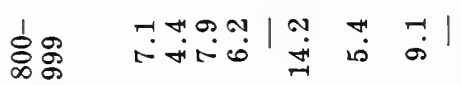

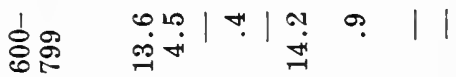

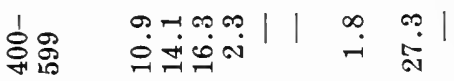

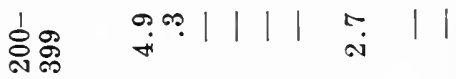
总

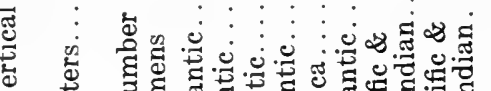
要 1. $\frac{1}{20}$

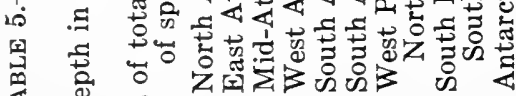

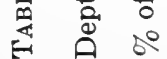

悹+

紊密

毫离

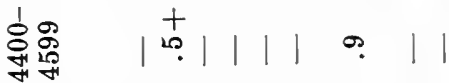

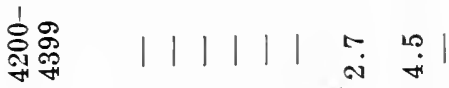

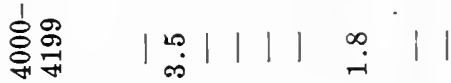

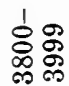

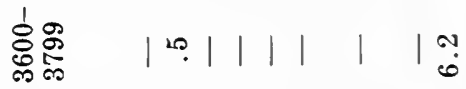

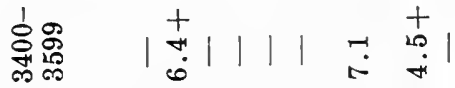

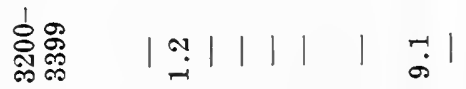

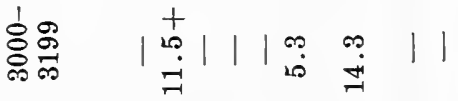

总兽|r|||| | | |

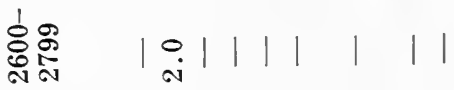

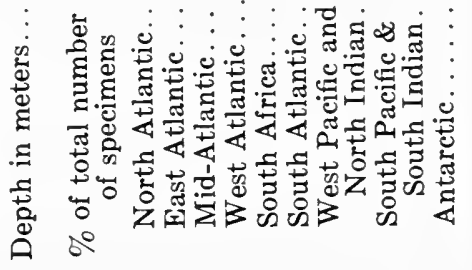


鄗多|||||

ฟูง

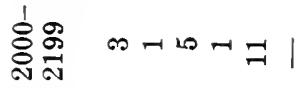

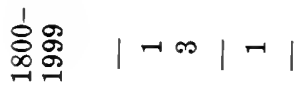

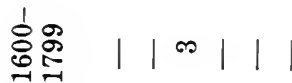

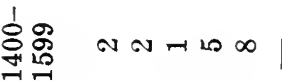

高嵒 $-1+\mid \infty$

亳 | | N

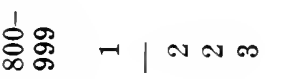

형욜|-|||

후욤

.

용

畺
퐁 뚜ㅇㅛㅛ

总+ | | N | | |

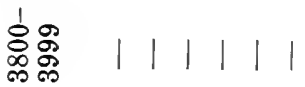

亮趸 | | | | | |

憝总 N | | | | |

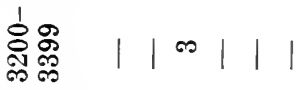

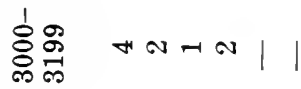

商昫 | | | | | |

高畓 | | | | | |

亭总 N

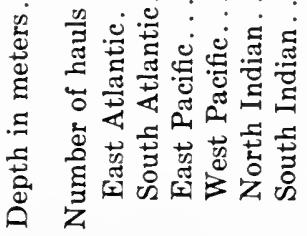

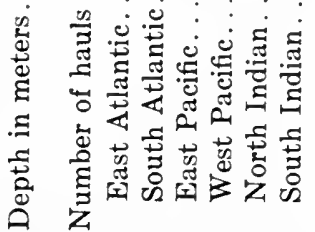




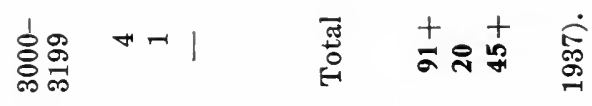

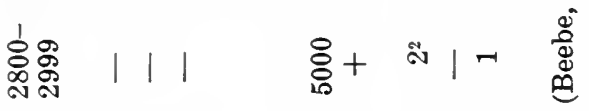

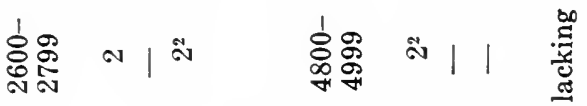

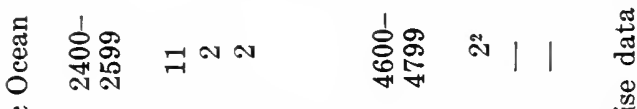

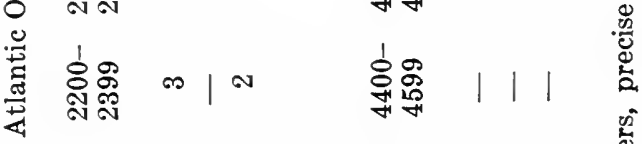

竎密

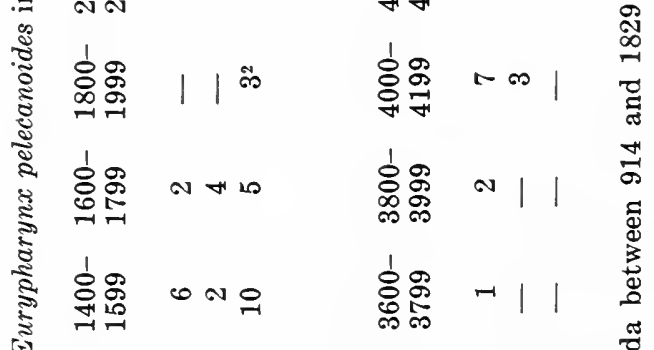

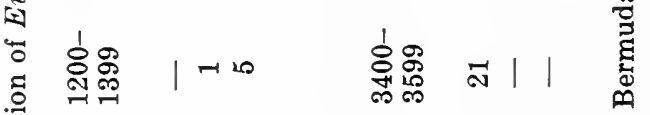

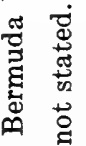

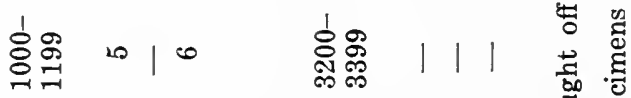

कo

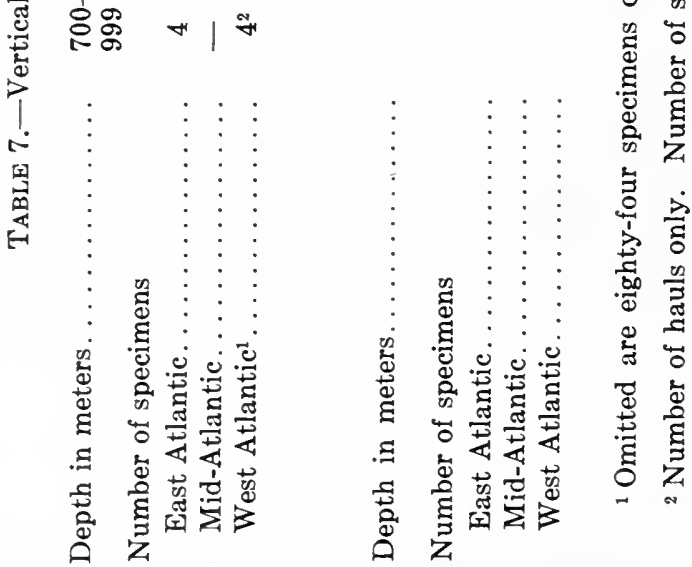




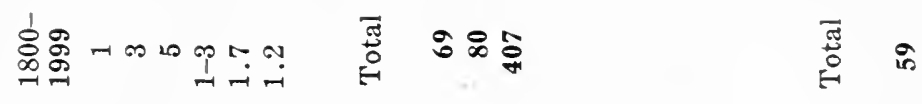

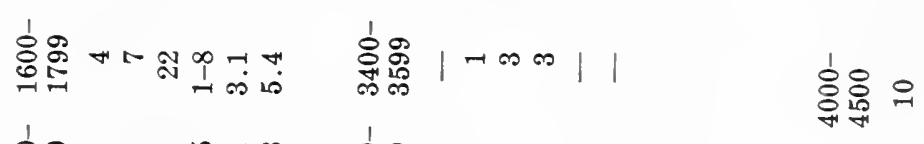

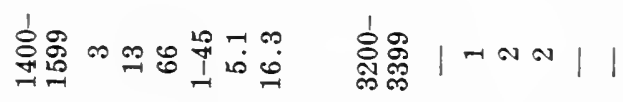

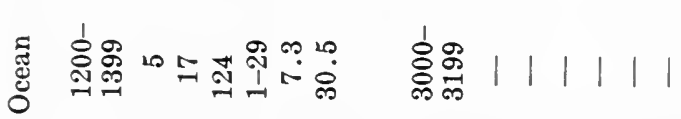

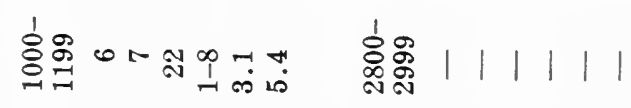

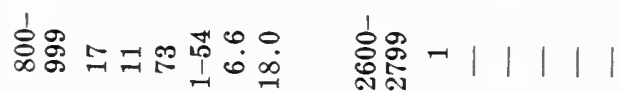

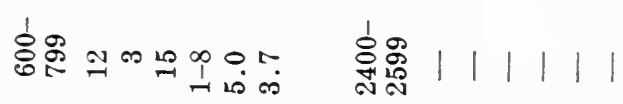

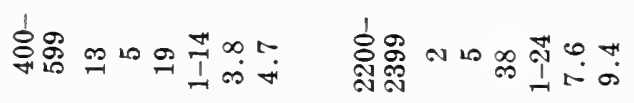

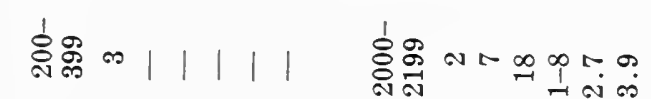
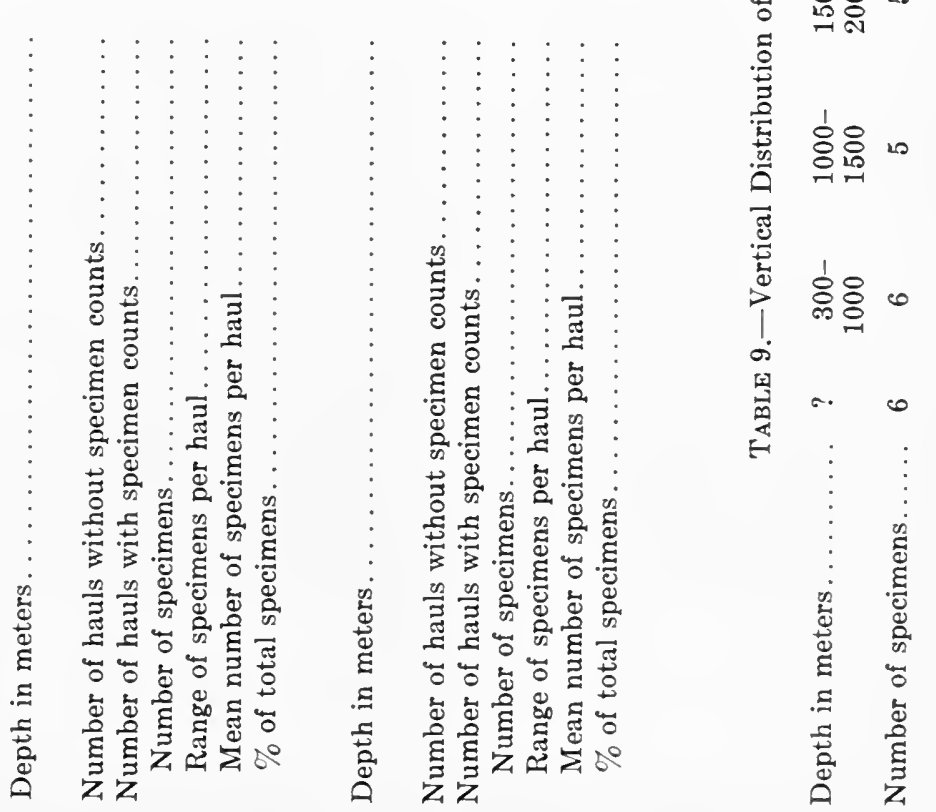


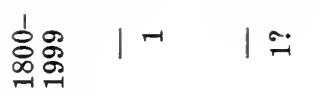

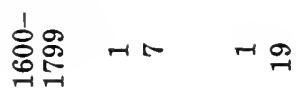

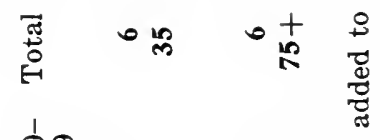

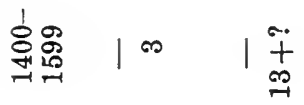

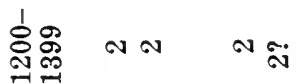
䱛1010<smiles></smiles><smiles>C[131I-]</smiles>
क्ष

尊

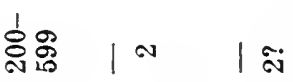

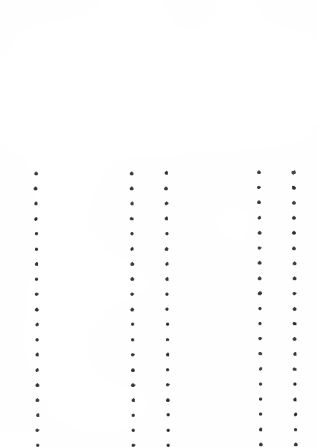

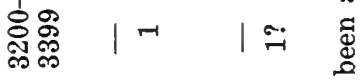

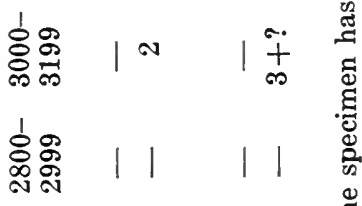

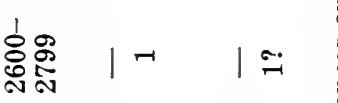

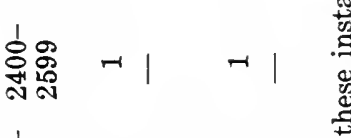
率哭

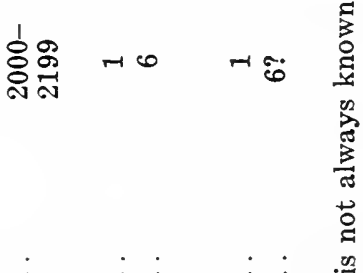

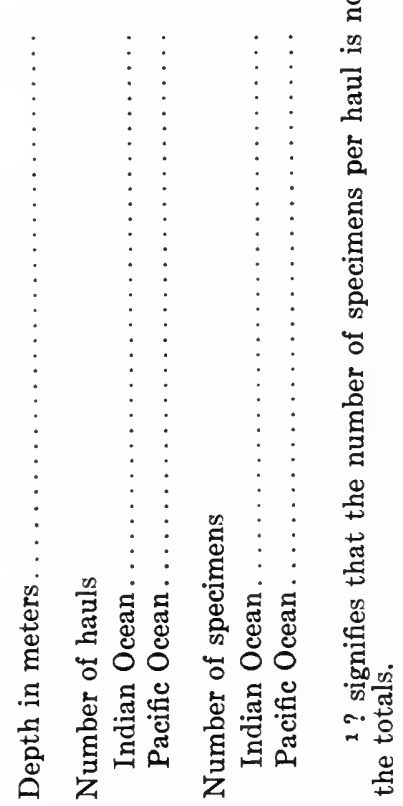




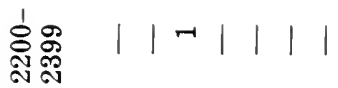

워ำ $-|-|$ |

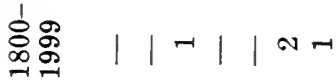

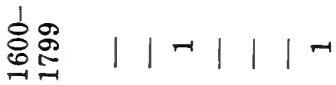

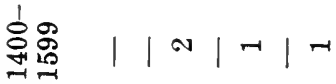

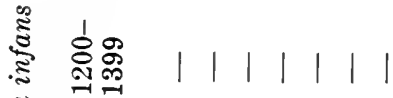

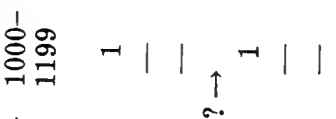

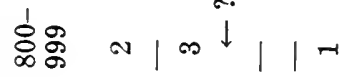

毫量 $-|-||-|$

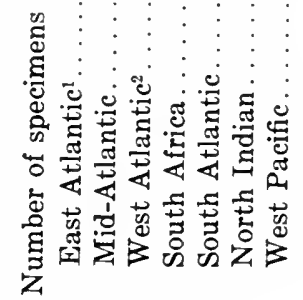

焉

号+

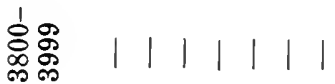

這盆 | | | | |

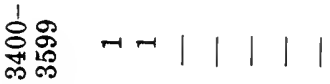

商总 | | | | | |

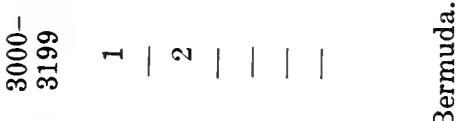

요 $|+|-1 \mid-1$

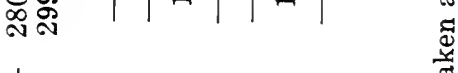

嗃冬 | | | | | |

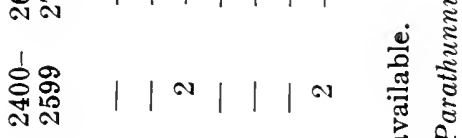

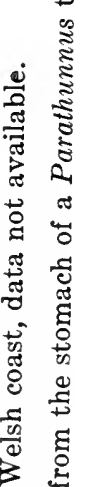

胥

๓ $\vdots \vdots \vdots \vdots \vdots$ च

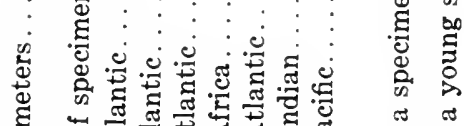

घ

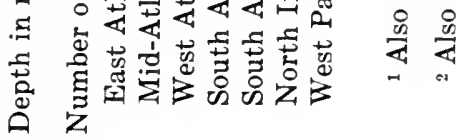




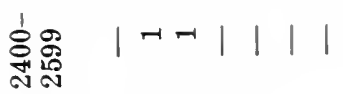

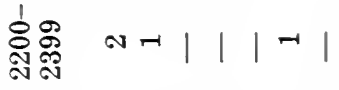

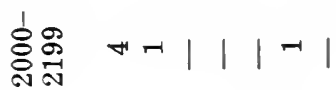

离㖏 | | | | | | |

兽涅 $-1|1|||$

害旁昫 -||$-|| \mid$

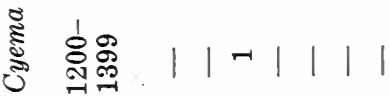

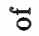

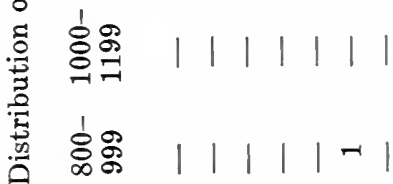

政
喜 ミかッーカーー

$\delta_{+}+-\pi|1| 1 \mid$

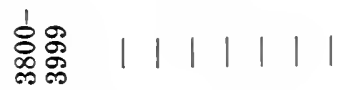

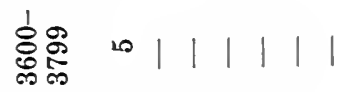

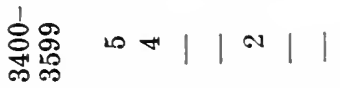

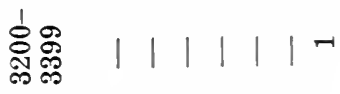

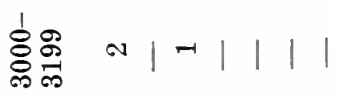

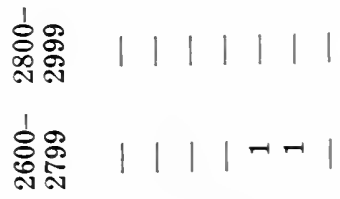

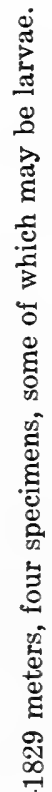

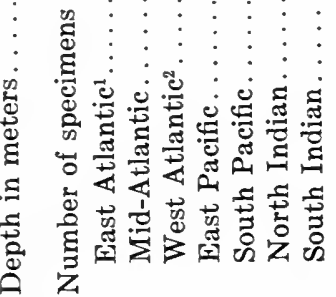

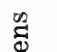

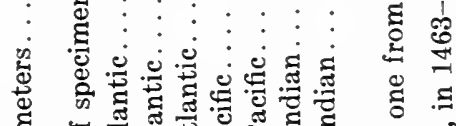

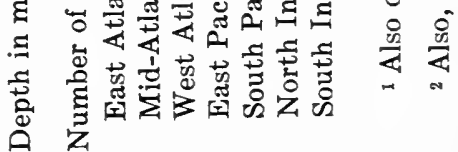




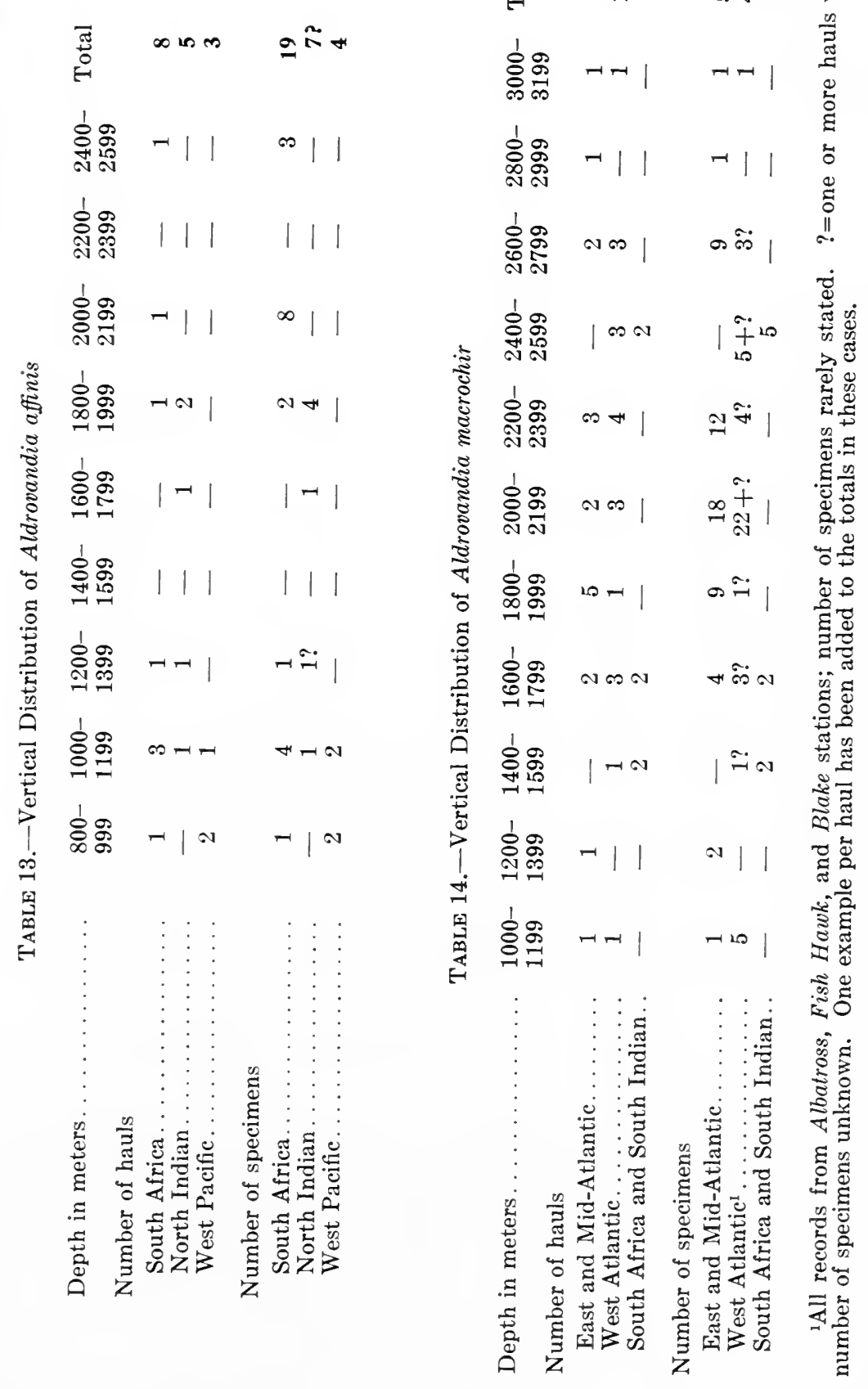




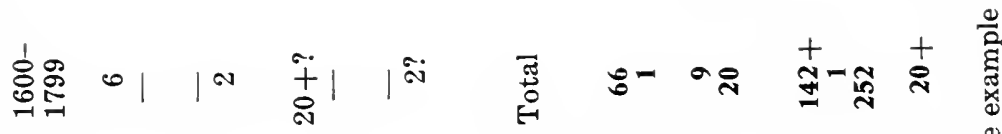

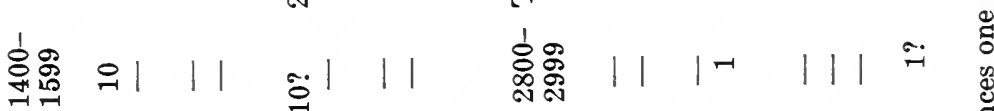

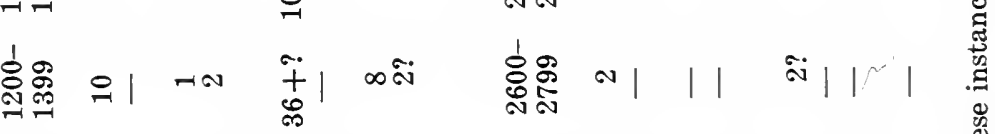

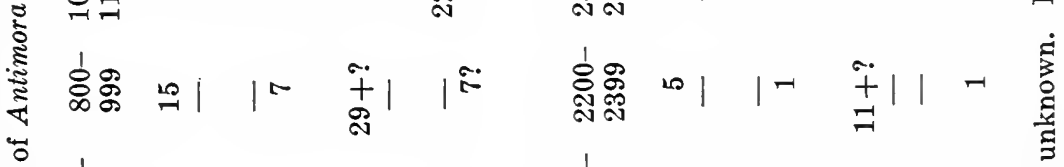

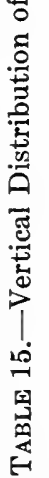

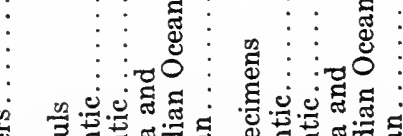

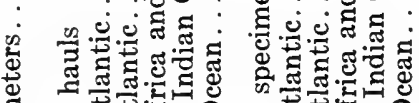

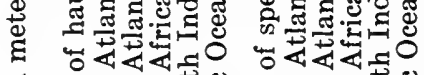

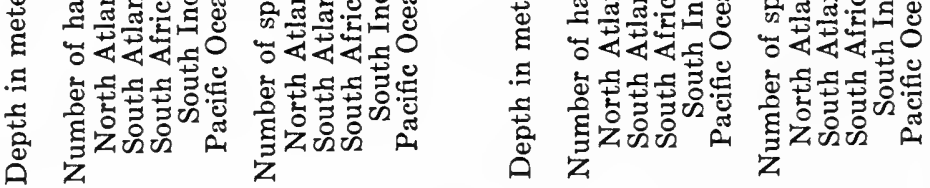
家总 N | |
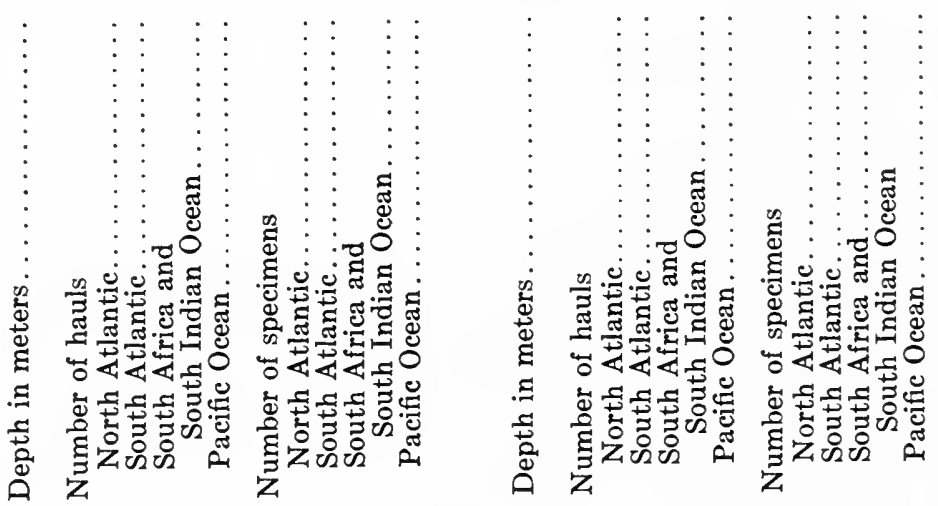

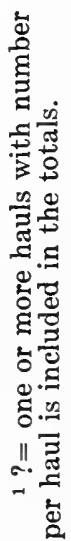




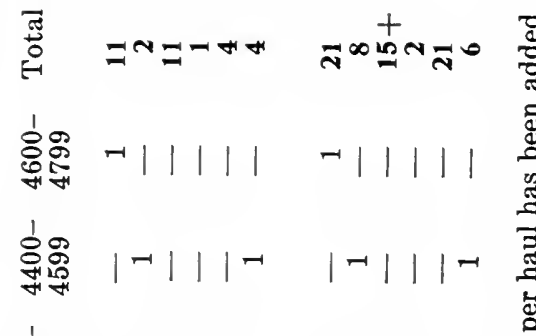

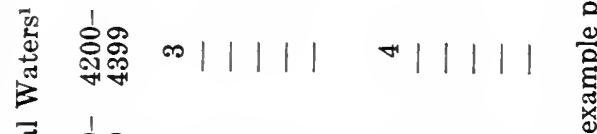

裹鮥-11111- -11111

敦

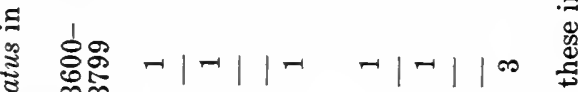

క్ల

8

政

है

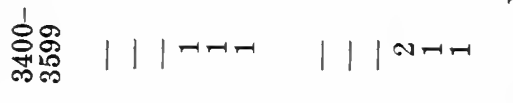

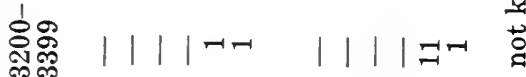

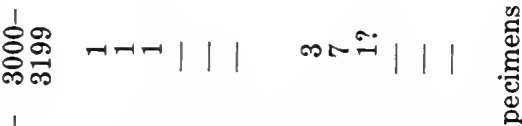

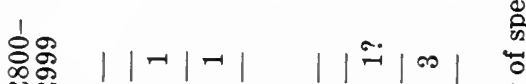

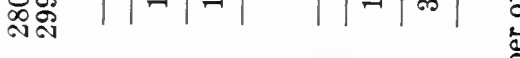

לํํ요 $\infty|n|||$ 엥 $\mid$ |

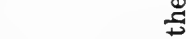

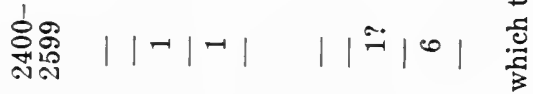

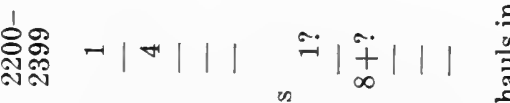

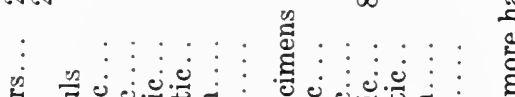

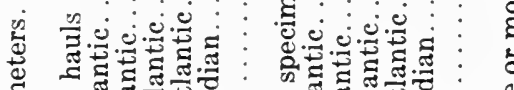

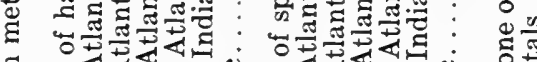

I $4<4$

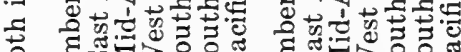

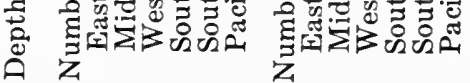

$\prod_{\substack{0 \\ 0}}^{\infty}$

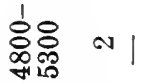

文需 -1

용요

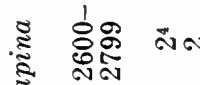

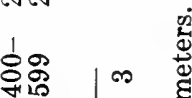

ลำ

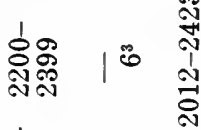

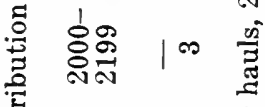

范

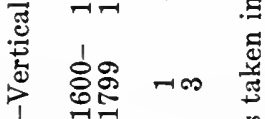

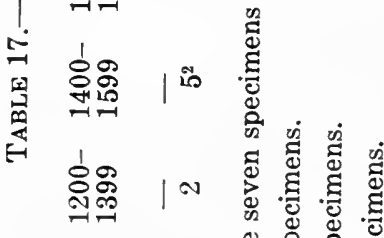

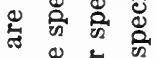

.

告

$\because 2$

웅 


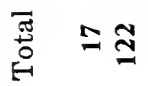

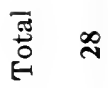

总 $r-r$

高怒| |

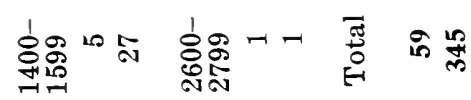

함웜

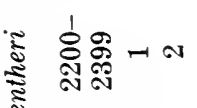

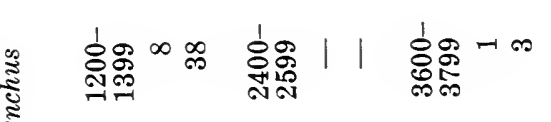

호요용

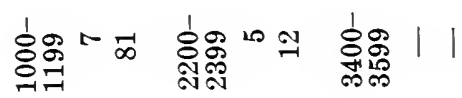

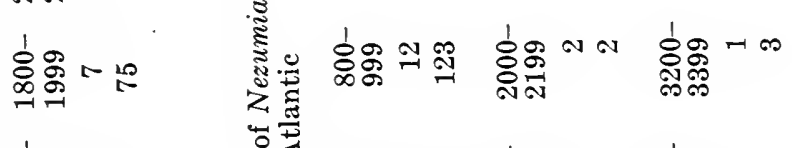

递题 $\cdots$

就山

త్

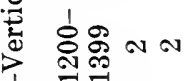

한

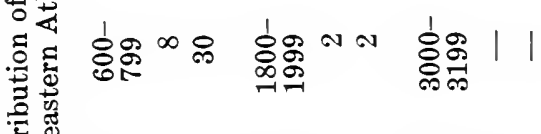

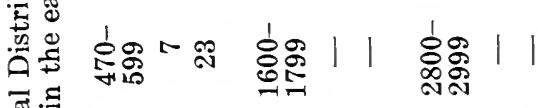

焉

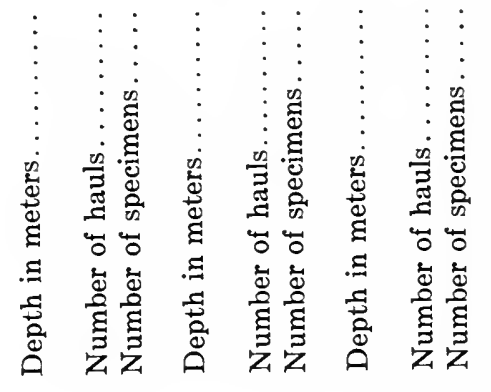

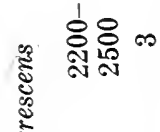

(4)

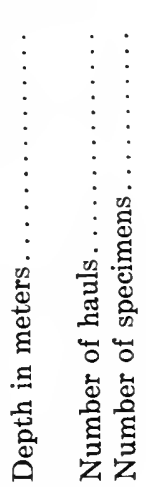

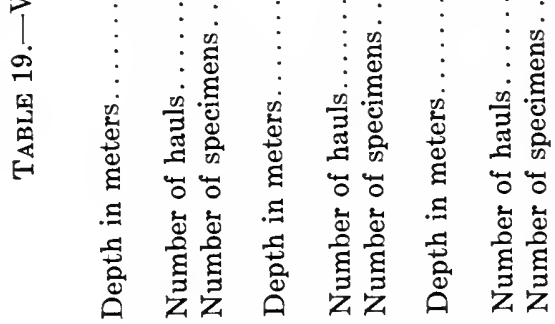




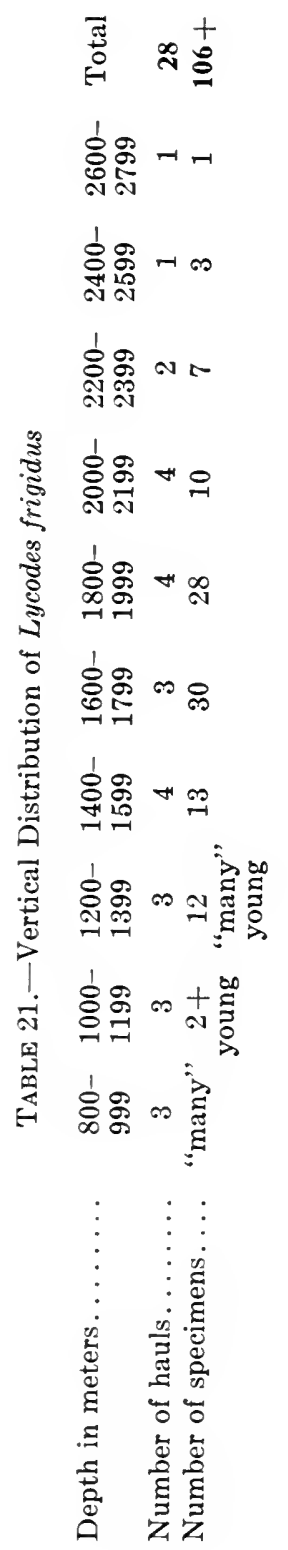

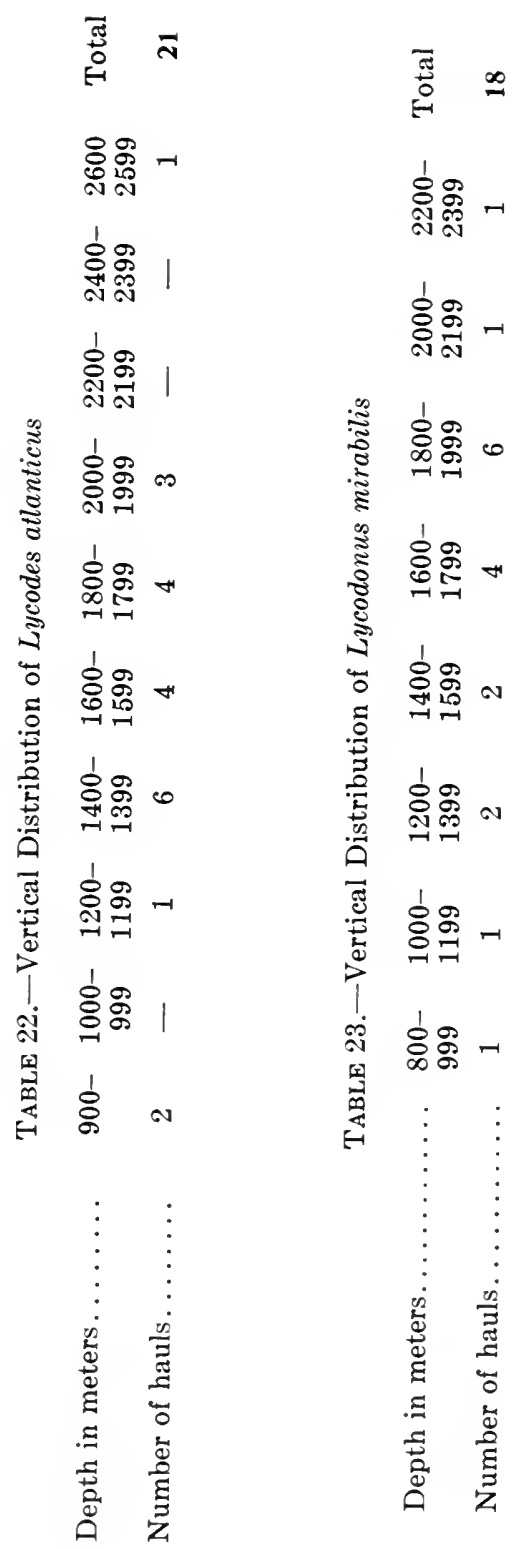



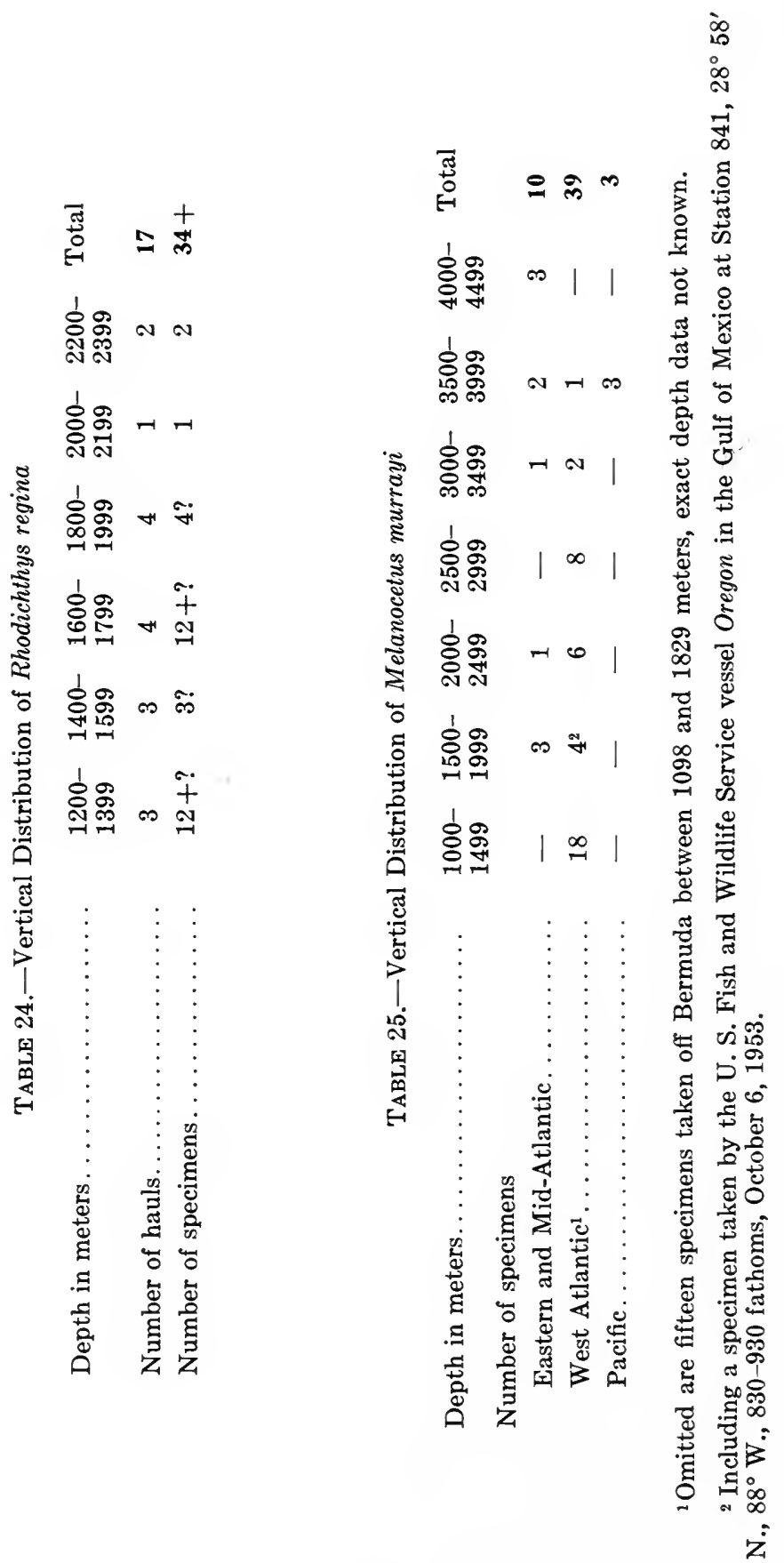


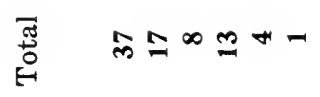

离总高 N | | | | |

亳旁 -|||| $\mid$

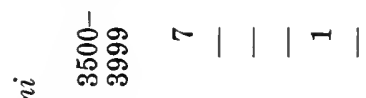

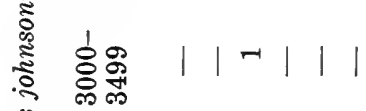

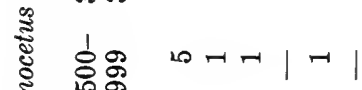

胥

\&

유융

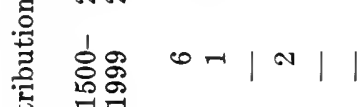

$\frac{n}{0}$

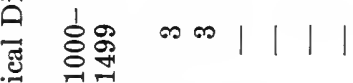

变

| 용용유 ง

四
要

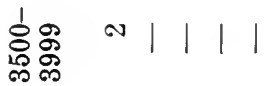

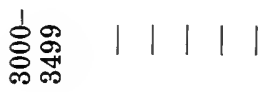

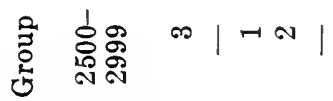

芯芯梁

胥

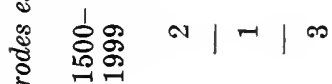

ङ

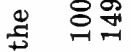

응요 $N \mid N-$

葛

帛员

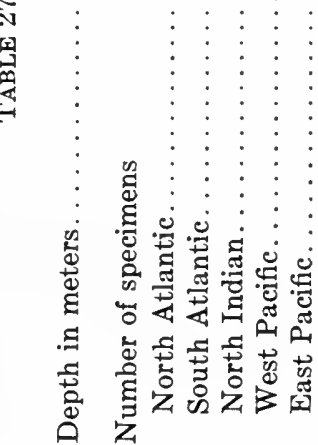




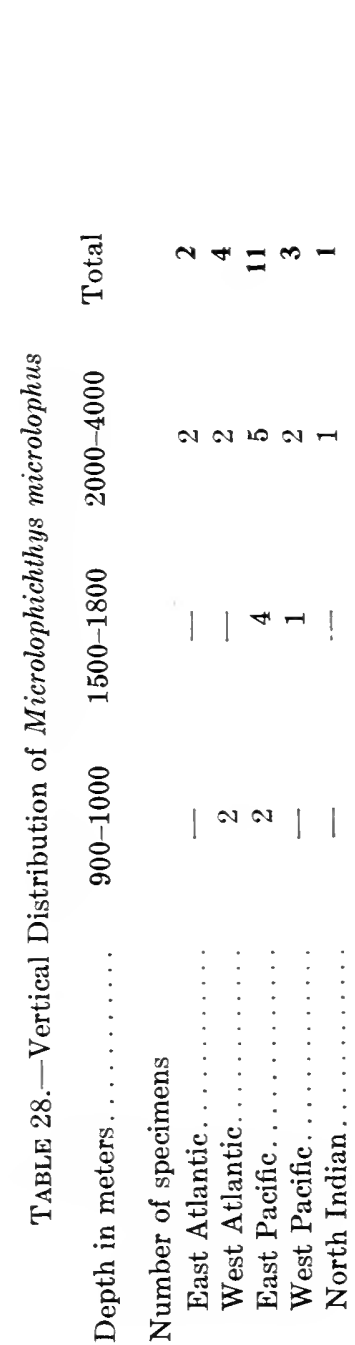

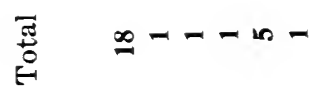

亲皮|1|1

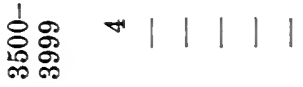

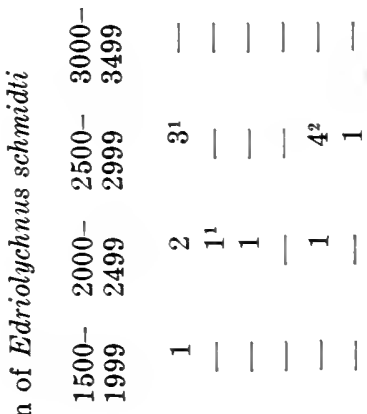

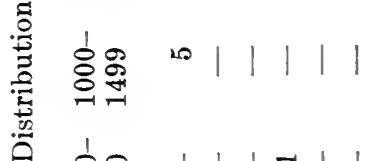

홍요

帘

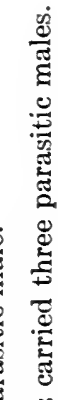

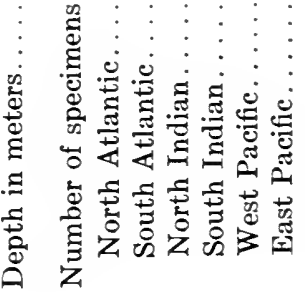

है थ

$\rightarrow$ है

兘

ฮี

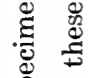

당

参 


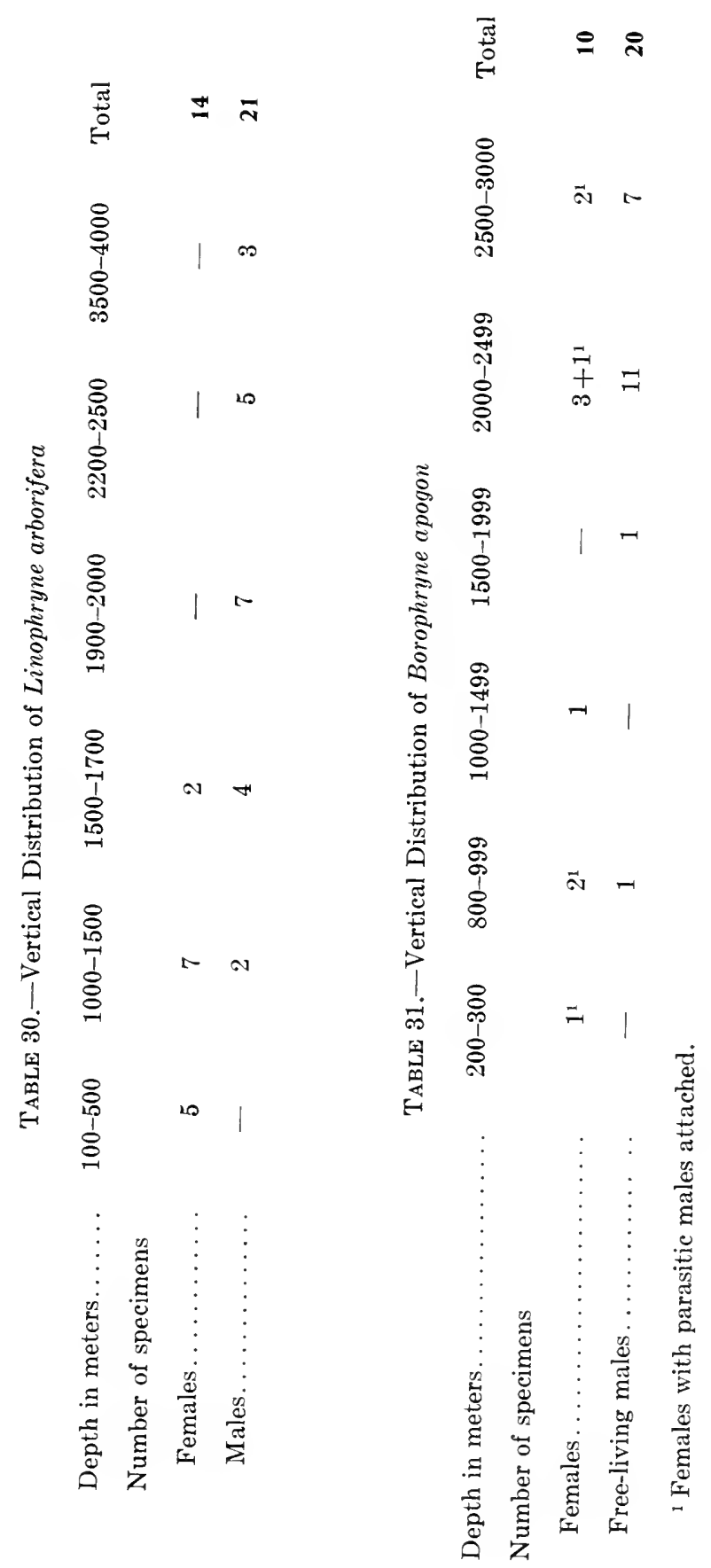




\section{BENTHIC FISHES FOUND BELOW A DEPTH OF 2000 METERS OR 1000 FATHOMS}

Names followed by an asterisk are those of species found also below 3660 meters (2000 fathoms).

Names followed by a dagger are those of species probably occurring normally in deep-abyssal waters.

\section{Key to Symbols Used for Showing Geographic Distribution}

$\begin{array}{llll}\text { AR } & \text { Arctic } & \text { EP } & \text { Eastern Pacific } \\ \text { EA } & \text { Eastern Atlantic } & \text { NP } & \text { North Pacific } \\ \text { M } & \text { Mediterranean } & \text { MP } & \text { Mid-Pacific } \\ \text { MA } & \text { Mid-Atlantic } & \text { WP } & \text { Western Pacific } \\ \text { SAf } & \text { South Africa } & \text { NI } & \text { North Indian } \\ \text { WA } & \text { Western Atlantic } & \text { SI } & \text { South Indian }\end{array}$

SWP Southwestern Pacific

SMP South mid-Pacific

SEP Southeastern Pacific

SA South Atlantic

AN Antarctic

Name
Apristurus indicus Brauer.

Distribution

Depth in meters

\section{Scylliorhinidae}

$1225-1840$

\section{Squalidae}

Etmopterus princeps Collett............. EA, WA

$567-2055$

Centrophorus squamosus Bonnaterre.......... EA

Centroscymnus coelolepis Bocage and Capello†... . EA, M, WA

400-1875

330-2718

\section{Rajidae}

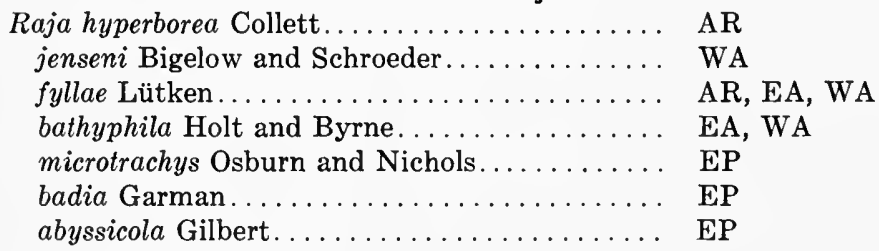

$183-2394$

$366-2296$

274-2055

$676-2173$

1994

2323

2904

\section{Chimaeridae}

Hydrolagus affinis Capello.............. EA, WA

293-2360

purpurescens Gilbert.

MP

1750-1951

Rhinochimaeridae

Harriotta raleighana Goode and Bean........ EA, WA

\section{Alepocephalidae}

\begin{tabular}{|c|c|c|}
\hline Alepocephalus rostratus Risso*............... & EA, M, ?WA & $300-3655$ \\
\hline agassizi Goode and Bean $\dagger .$. & EA, WA & $984-2286$ \\
\hline productus Gill... & EA, WA & $2055-2491$ \\
\hline fundulus Garman. & $\mathrm{EP}$ & $2322-3057$ \\
\hline australis Barnard.. & ?EA, SAf & $1153-2603$ \\
\hline
\end{tabular}


Name

atlanticus Roule and Angel'.

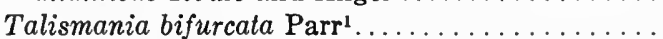

Rinoctes nasutus Koefoed*................

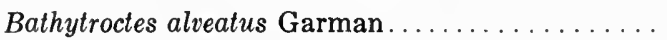

macrolepis Günther*...............

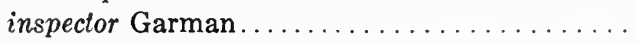

alvifrons Garman.

koefoedi Parr....

michaelsarsi Koefoed.

Bajacalifornia drakei Beebe ${ }^{1}$.

Grimatroctes grimaldii Zugmayer ${ }^{1}$ microlepis Günther.

Narcetes pappenheimi Fowler. stomias Gilbert.

pluriserialis Garman.

affinis Lloyd

Bellocia vaillanti Parr.

Leptochilichthys macrops Roule and Angel ${ }^{1}$ agassizi Garman

Rouleina attritus Vaillant* $\dagger$ danae Parr ${ }^{1}$. guentheri Alcock.

Asquamiceps velaris Zugmayer ${ }^{1}$. indagatio Parr ${ }^{1}$. pacificus Parr ${ }^{1}$.

Brunichthys asperifrons Garman

Leptoderma macrops Vaillant.

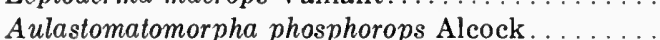
caeruliceps Lloyd.

Conocara werneri Nybelin macroptera Vaillant $\dagger$.

murrayi Koefoed

Ericara salmonea Gill and Townsend* niger Günther.

Einara macrolepis Koefoed ${ }^{1}$.

\section{Searsiidae}

Mirorictus taningi Parr ${ }^{1}$.

Platytroctes apus Günther

Holtbyrnia melanocephalus Vaillant.

Searsia polycoeca Parr ${ }^{1}$.

Pellisolus facilis Parr ${ }^{1}$

Barbantus curvifrons Roule and Angel' ${ }^{1}$.
Distribution

EA

EP

EA, WA

EP

WP, ?NI

EP

EP

EA

EA

WA

EA

EA, ?NI

WP

$\mathrm{EP}$

EP

NI

EA

EA

EP

EA

WP

NI

EA

SAf

EP

EP

EA

NI

NI

EA

EA, WA

EA, WA

EP, NP

WP

EA
Depth in meters

4000

$? 2200$

2865-3886

2068-2415

3932

2690

2488-3279

2865

2865

732-1645, ?2012

4900

$1992, ? 914$

1997

1604-1968

1847

1839

2600

3000

2877

1442-3655

$? 2500$

786-2140

3660

2580

2200

1430-1865

800-2330

1829-2012

1839

2150-2300

865-2115

2055-2603

2469-3991

2560

4000

$? 2200$

EP

1353-2744

$? 4900$

EA, ?WA

EA, WA

EP

EA

?-2012

?2300

4500

\section{Bathylaconidae}

Bathylaco nigricans Goode and Bean*.

WA, EP

2200-4370

${ }^{1}$ Perhaps pelagic. 
Name

Distribution

Depth in meters

Bathysaurus ferox Günther $\dagger$.

Harpadontidae mollis Günther*†

EA, MA, SAf, 1098-3120 WA, SWP

EA, EP, WP, 2615-4361 SMP

\section{Chlorophthalmidae}

Bathysauropsis gracilis Günther $\dagger$

SAf, SWP, SEP, SA

869-2606

Bathypteroidae

Benthosaurus grallator Goode and Bean $\dagger . . . . . .$.

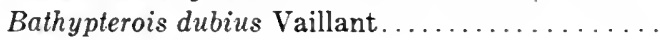

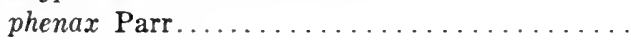

EA, WA

2104-3384

EA, M

$800-1940$

WA

$1645-2194$

antennatus Gilbert.

MP, WP

258-2403

pectoralis Garman.

EP

1619-2070

longipes Günther*

EA, WA, SA

$827-4846$

filiferus Gilchrist.

SAf

insularum Alcock

NI

1098-2561

capensis Gilchrist and von Bonde

SAf

2084

2231

\section{Ipnopidae}

Bathymicrops regis Hjort and Koefoed ${ }^{*} \dagger \ldots . .$. seuelli Norman*.

EA, WA

NI

4255-5300

SAf, WA, WP,

NI, SA

agassizi Garman

EP

$3840-3872$

Ipnops murrayi Günther* $\dagger$

WP

2488

1393-1992

Simenchelyidae

Simenchelys parasiticus Gill

EA, SAf, WA,

$366-2620$ WP

Nettastomidae

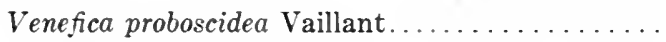

ocella Garman.......................

procera Goode and Bean.

EA, SAf, NI

EP

$1207-2200$

1951

WA, WP

326-2194

Congridae

Promyllantor purpureus Alcock

NI

1829

Ilyophidae

Ilyophis brunneus Gilbert.

EA, SAf, WA,

1159-2615

\section{Synaphobranchidae}

Synaphobranchus infernalis Gill $\dagger$.
bathybius Günther* ${ }^{*} . \ldots \ldots \ldots$ 


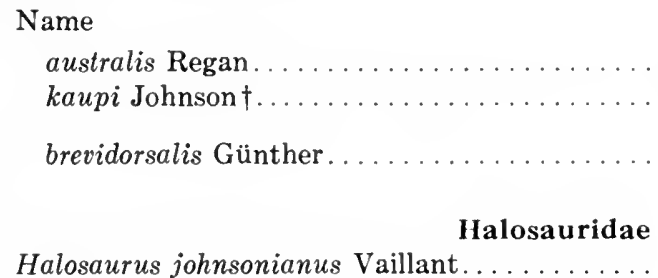

Depth in

Distribution meters

SI, SA

2515-3185

EA, SAf, WA

236-3200

?3465

WP, NI

693-1956

attenuatus Garman................. EP

Aldrovandia rostrata Günther* ...........

affinis Günther $\nmid \ldots \ldots \ldots \ldots \ldots \ldots \ldots \ldots$

EA, MA, ?WA

$834-2115$

EA

2488

$? 1180$

SAf, WP, NI

1311-5029

WA

$883-2560$

gracilis Goode and Bean.

WA

1380-2615

pallida Goode and Bean.

EA, MA, SAf,

1241-2615

macrochir Günther $\dagger$

WA, SI

1163-3166

phalacra Vaillant $\dagger$

EA, NI

1103-2320

\section{Notacanthidae}

Macdonaldia sp. Koefoed................ EA

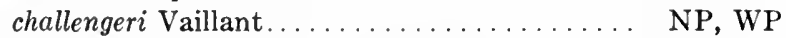

africana Gilchrist and von Bonde......... SAf

Polyacanthonotus vaillanti Fowler............ EA, WA

altus Gill and Townsend.............. NP

2615

2971-3429

2231

$1995-2212$

2562

\section{Moridae}

Lepidion lepidion Risso .

EA, M, WA

540-2230

EA, SAf, WA,

EP, NP, MP,

403-2904

WP, SWP, SI,

SA

Laemonema melanurum Goode and Bean...... EA, WA

$380-2683$

\section{Gadidae}

Gaidropsarus ensis Reinhardt

WA

$360-2022$

\section{Macrouridae}

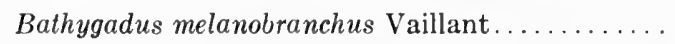

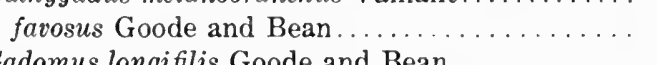

Gadomus longifilis Goode and Bean.

Nematonurus cyclolepis Gilbert.

lecointei Dollo.

suborbitalis Gill and Townsend.

EA, SAf

$830-2560$

?EA, WA

$768-2745$

EA, WA

$631-2165$

EP

2904

SA, AN

NP

2800-3246

3239

2065-2562

NP

abyssorum Gilbert*................. EP

armatus Hector* $\dagger$

EA, MA, WA,

MP, SWP, SI,

2469-3991

SA

EA, WA

282-4700

Coryphaenoides rupestris Gunnerus.

EA, SWP

155-2276

$\mathrm{M}$ 
Name

Distribution

fernandeziana Günther.

SEP

Depth in

meters

filifera Gilbert.

EP

2515

2904

liocephala Günther*.

MP, WP

$3429-3749$

simula Goode and Bean*†

EA, MA, WA

carapina Goode and Bean* $\dagger$

EA, MA, WA

$1669-4600$

(?610-1183)

ferrieri Regan.

AN

1211-5300

whitsoni Regan.

$\mathrm{SA}, \mathrm{AN}$

2579-3109

guentheri Vaillant $\dagger$.

EA, ?NI

604-3185

serrula Bean.

NP

$1200-2603$

brevibarbis Goode and Bean*†.

EA, MA, WA

2869

Macrourus paradoxus Smith and Radcliffe

WP

$1748-4700$

2021

bucephalus Garman................. EP

carminifer Garman................. EP

anguliceps Garmant................ EP

longicirrhus Gilbert................. MP

aequatoris Smith and Radcliffe........... WP

cinereus Gilbert................... NP

altipinnis Günther................ WP

ingolfi Lütken................... EA

sublaevis Vaillant................. EA

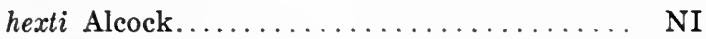

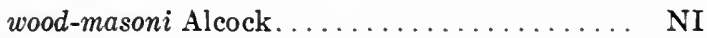

hoskyni Alcock. ................... NI

camurus Smith and Radcliffe............ WP

245-2877

$588-1865$

$1271-2417$

$1829-2403$

$1992-1997$

$630-1890$

$1034-3429$

$1545-2378$

$$
\text { ? }
$$

$1582-1829$

$693-1840$

2395

2021

2308

3429

1968

albatrossus Townsend and Nichols......... EP

Hemimacrurus acrolepis Bean $\nmid \ldots \ldots \ldots \ldots \ldots$ EP, NP, WP

$155-2226$

(?2469)

Grenurus hirundo Collett.............. EA

$1266-2300$

flagellicauda Koefoed.

EA, MA

$2150-3120$

Oxygadus labiatus Koehler................. EA

$460-2220$

Hymenocephalus italicus Giglioli........... EA, M

$400-4900$

Lionurus filicauda Günther*†.

SI, SEP, SA

$2515-4846$

294-2012

liolepis Gilbert.

EP

fragilis Garman.

EP

Nezumia convergens Garman............. EP

bairdi Goode and Bean............... ?EA, WA

$3057-3333$

$1271-1865$

$0-2295$

aequalis Günther.

EA, M, WA

$200-2320$

sclerorhynchus Valenciennes* $\dagger$

EA, M

parvipes Smith and Radcliffe $\dagger . . . . . \ldots \ldots$ WP

500-3655

EA, WA

$1992-2308$

Cetonurus globiceps Vaillant*.

EA

$1139-4621$

$5000-5413$

\section{Stephanoberycidae}

Stephanoberyx monae Gill

WA

979-2295

Acanthochaenus luetkeni Gill*†.

EA, WA

Malacosarcus macrostoma Günther*.......... MP 
Name

Distribution

\section{Acropomatidae}

Brephostoma carpenteri Alcock.............. NI

\section{Parapercidae}

Macrias amissus Gill and Townsend......... SEP

Bathydraconidae

Bathydraco antarcticus Günther.

AN

AN

2304

$2260-2579$

\section{Zoarcidae}

Lycodes frigidus Collett $\nmid \ldots \ldots \ldots \ldots \ldots \ldots \ldots$ AR, SAf

atlanticus Jensen $\dagger \ldots \ldots \ldots \ldots \ldots \ldots \ldots \ldots \ldots$ WA

platyrhinus Jensen.................. AR

cicatrifer Garman................... EP

Lycenchelys jordani Evermann and Goldsborough. NP

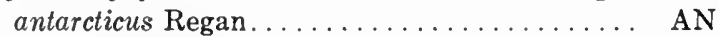

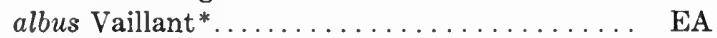

Lycodonus ophidium Jensen............... EA

mirabilis Goode and Beant............. WA

flagellicauda Jensen.................. AR

Maynea bulbiceps Garman............... EP

conorhynchus Garman............... EP

Bothrocaropsis elongata Garman............ EP

Melanostigma pammelas Gilbert............ EP

atlanticum Koefoed.................. EA, SAf, WA

Pachycara obesa Zugmayer*. meters

$2505-2780$

1920

$631-2750$

944-2603

1847

3057

$1687-1945$

3246

3975

1992

$850-2394$

$839-1835$

2690

3279

1271-1865

97-2012

723-1853

(?2561)

4780
Depth in

(?2880-3000)

111-1968

EP, NP

2904

NP

\section{Brotulidae}

Monomeropus malispinosus Garman..........

Dicrolene filamentosa Garman..............

nigra Garman....................

Barathrodemus manatinus Goode and Bean..... nasutus Smith and Radcliffe.............

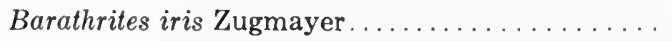
abyssorum Roule*..................

Bassogigas crassus Vaillant*†............... pterotus Alcock..................... aequatoris Smith and Radcliffe............. digittatus Garman* $\dagger$

EP

1865

EP

935-1865

$769-1865$

EP

1183-2552

$1992-1997$

WP

MA, WA

1645-3465

5285

EA

EA, MA

1797-4255

$1829-3197$

1992

$2196-4082$ 


\begin{tabular}{|c|c|c|}
\hline Name & Distribution & $\begin{array}{l}\text { Depth in } \\
\text { meters }\end{array}$ \\
\hline 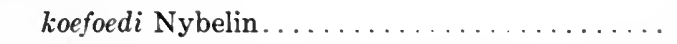 & EA & 2603 \\
\hline brucei Dollo*............ & $\mathrm{AN}$ & 4571 \\
\hline grandis Günther................... & WP & 3465 \\
\hline 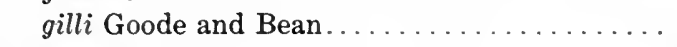 & WA & 2022 \\
\hline Alcockia rostrata Günther ${ }^{*} \ldots \ldots \ldots \ldots \ldots$ & WP & 3932 \\
\hline Bassozetus normalis Gill $\uparrow \ldots . . . \ldots \ldots \ldots \ldots$ & WA & $2068-3512$ \\
\hline 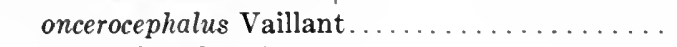 & $\mathrm{EA}$ & 3200 \\
\hline 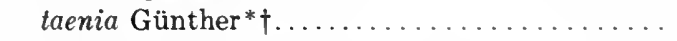 & MA, WA & $4571-5610$ \\
\hline 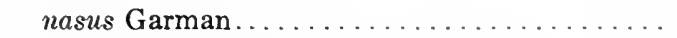 & $\mathrm{EP}$ & $3057-3436$ \\
\hline 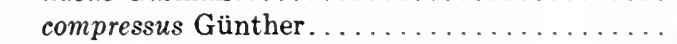 & MA, WP & $1920-2744$ \\
\hline elongatus Smith and Radcliffe............. & WP & 1992 \\
\hline glutinosus Alcock $† \ldots \ldots \ldots \ldots \ldots \ldots \ldots$ & NI & $1163-2395$ \\
\hline Eretmichthys pinnatus Garman... & $\mathrm{EP}$ & $1789-2417$ \\
\hline ocella Garman............. & EP & 2322 \\
\hline 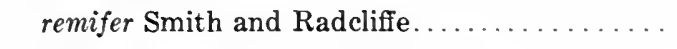 & WP & 1997 \\
\hline Porogadus miles Goode and Bean............ & SAf, WA & $1280-2194$ \\
\hline 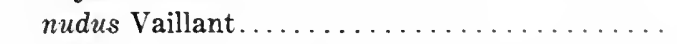 & $\mathrm{EA}$ & $2324-3200$ \\
\hline 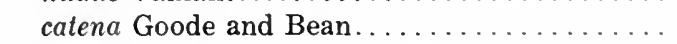 & WA & 2683 \\
\hline 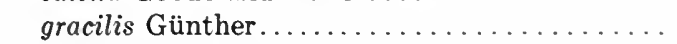 & WP & 2560 \\
\hline$s$ Alcock $† \ldots \ldots \ldots \ldots \ldots \ldots \ldots$ & $\mathrm{NI}$ & $1336-2312$ \\
\hline melanocephalus Alcock................. & $\mathrm{NI}$ & $3006-3197$ \\
\hline subarmatus Vaillant $\dagger \ldots . . . . . . . . . . . . .$. & EA, WA & $2104-3200$ \\
\hline 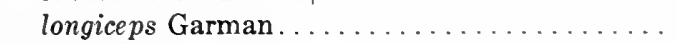 & $\mathrm{EP}$ & $1865-3279$ \\
\hline atripectus Garma & EP & $1412-2322$ \\
\hline 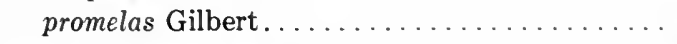 & EP & $1180-1968$ \\
\hline Penopus macdonaldi Goode and Bean.......... & WA & 2982 \\
\hline Penopus(?) microphthalmus Vaillant....... & $\mathrm{EA}$ & 3200 \\
\hline Mixonus laticeps Günther*........ & EA, MA, ?WA & $2615-4571$ \\
\hline caudalis Garman $\dagger \ldots . .$. & $\mathrm{EP}, \mathrm{NI}$ & $1524-2417$ \\
\hline 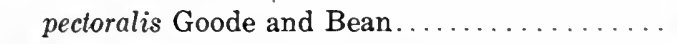 & WA & 604,2615 \\
\hline Mastigopterus praetor Smith and Radcliffe....... & WP & 2361 \\
\hline Grimaldichthys squamosus Roule*........... & $\mathrm{EA}$ & 4621 \\
\hline profundissimus Roule*................ & MA, WA & $5600-6035$ \\
\hline Leucicorus lusciosus Garman................ & $\mathrm{EP}$ & 3436 \\
\hline Enchelybrotula paucidens Smith and Radcliffe... & WP & 1992 \\
\hline Acanthonus armatus Günthert............ & EA, WP & $1920-2603$ \\
\hline 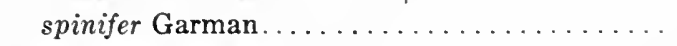 & $\mathrm{EP}$ & 3240 \\
\hline Tauredophidium hexti Alcock............. & NI & 2395 \\
\hline Typhlonus nasus Günther*†..... & WP & $3932-4461$ \\
\hline Cataetyx simus Garman........ & $\mathrm{EP}$ & $2322-2690$ \\
\hline Diplacanthopoma brunnea Smith and Radcliffe... & WP, NI & $685-1893$ \\
\hline Aphyonus gelatinosus Günther.............. & WP & 2560 \\
\hline Sciadonus pedicellaris Garman........ & EP & 1847 \\
\hline Barathronus affinis Brauer........ & NI & 2919 \\
\hline 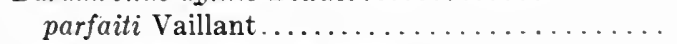 & $\mathrm{EA}$ & 1846,5005 \\
\hline Leucochlamys cryptophthalmus Zugmayer*...... & EA & 5000 \\
\hline
\end{tabular}


Name

Distribution

Depth in meters

Cottidae

Zesticelus profundorum Gilbert

$\mathrm{EP}, \mathrm{NP}$

$730-1980$

Cottunculidae

Cottunculoides spinosus Gilchrist............ SAf

$1463-2176$

\section{Liparidae}

Rhodichthys regina Collett $\uparrow$

AR

$1150-2365$

Careproctus bathycoetus Gilbert and Burke.

NP

3291

3333

longifilis Garman.

EP

2904

ovigerum Gilbert................. EP

1914

opisthotremus Gilbert and Burke.

NP

3240

Paraliparis fimbriatus Garman

EP

ulochir Gilbert

EP, NP

bathybii Collett.

AR

latifrons Garman

EP

$743-1838$

$670-2000$

3279

holomelas Gilbert.

NP

$128-3350$

copei Goode and Bean

EA, SAf, WA

548-1829

2904

grandiceps Garman.

EP

1248-3608

\section{Chaunacidae}

Chaunax roseus Barbour

WA

1956

Ogocephalidae

Malthopsis spinosa Garman.............. EP

1865-2322

EP

2417-2488

WP, NI

343-1886

Dibranchus nasutus Alcock.

NI

1789-2012

$\mathrm{EP}$

$1153-2322$

hystrix Garman.

NI

1022-1840

\section{BATHYPELAGIC FISHES FOUND BELOW A DEPTH OF 2000 METERS OR 1000 FATHOMS}

\section{Gonostomatidae}

\begin{tabular}{|c|c|c|}
\hline Gonostoma bathyphilum Vaillant $\nmid \ldots . . . \ldots \ldots$ & $\begin{array}{l}\text { EA, MA, WA, } \\
\text { SAf }\end{array}$ & $650-5100$ \\
\hline Cyclothone livida Brauert.................. & $\mathrm{EA}, \mathrm{SA}$ & $500-4972$ \\
\hline 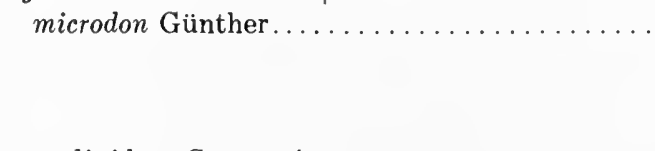 & $\begin{array}{l}\text { EA, M, MA, } \\
\text { SAf, WA, EP, } \\
\text { NP, WP, MP, } \\
\text { NI, SI, SWP, } \\
\text { SMP, SA, AN }\end{array}$ & $0-5000$ \\
\hline acclinidens Garman $\dagger$. & $\begin{array}{l}\text { EA, MA, SAf, } \\
\text { ?WA, EP, MP, } \\
\text { WP, NI, SA }\end{array}$ & $224-4417$ \\
\hline obscura Brauer $\dagger \ldots$ & EA, SAf, NI & $800-5248$ \\
\hline
\end{tabular}


Name
Eurypharyngidae
Eurypharynx pelecanoides Vaillant $\dagger \ldots \ldots \ldots \ldots$

Distribution

Depth in

meters

EA, MA, WA,

549-7625

EP, MP, WP,

NI, SWP

\section{Serrivomeridae}

Serrivomer parabeani Bertin $\nmid \ldots \ldots \ldots \ldots \ldots \ldots \ldots$ EA, MA, WA

$300-4500$

sector Garman $†$.

$\mathrm{EP}, \mathrm{MP}, \mathrm{NI}$,

245-3240

Nemichthyidae

Avocettina infans Günther $\nmid \ldots \ldots \ldots \ldots \ldots \ldots \ldots$ EA, MA, SAf,

$600-4571$

WA, WP, NI,

SA

Cyemidae

Cyema atrum Günther $\dagger$

EA, MA, SAf,

WA, EP, SEP,

$800-5100$ SWP, NI, SI,

SA

Melamphaidae

Melamphaes nigrescens Brauer $\dagger$.

EA, MA, SAf, WA, EP, WP, $1098-4000$ NI, SI

Triacanthodidae

Atrophacanthus danae Fraser-Brunner $† . . . . .$. WP

$300-3500$

Melanocetidae

Melanocetus murrayi Günther $\dagger$.

EA, MA, WA, EP, WP, SWP

Melanocetus johnsoni Günther $\dagger$

EA, MA, SAf,

WA, NI, WP,

$1050-4480$

SWP, EP

0-4789

\section{Oneirodidae}

Microlophichthys microlophus Regan $\dagger$

EA, WA, EP, WP, NI

$914-4000$

\section{Linophrynidae}

$\begin{array}{lll}\text { Edriolychnus schmidti Regant.................. } & \text { EA, MA, WA, } \\ \text { SA, EP, SWP, } & \text { NI }\end{array}$

Borophryne apogon Regan $† . . . \ldots \ldots \ldots \ldots \ldots$ EP

$200-4000$

200-2700 


\section{REFERENCES}

\section{Agassiz, Alexander}

1906. Reports on the scientific results of the expedition to the tropical Pacific .... V. General report of the expedition. Mem. Mus. Comp. Zool., 33, 75 pp., 96 pls., 8 text figs.

\section{Albert I, Prince of Monaco}

1932. Sur la septième campagne scientifique de la Princesse Alice; La mer des Sargasses. Rés. Camp. Sci. Monaco, 84: 37-41 (originally published in C. R. Acad. Sci., 142: 621-624, 1906).

Alcock, A.

1889. Natural history notes from H. M. Indian survey steamer Investigator. On the bathybial fishes of the Bay of Bengal and neighbouring waters, obtained during the seasons 1885-1889. Ann. Mag. Nat. Hist., (6), 4: 376-399; $450-461$.

1892. Natural history notes from H. M. Indian survey steamer Investigator. On the bathybial fishes collected during the season of 1891-92. Ann. Mag. Nat. Hist., (6), 10: 345-365, 1 pl., 2 text figs.

1899. A descriptive catalogue of the Indian deep-sea fishes. $220 \mathrm{pp}$. Calcutta, Indian Museum.

ANDRIASHEV, A. P.

1954. Fishes of northern seas [trans.]. Fauna S.S.S.R., 53, 566 pp., 300 figs. Akad. Nauk S.S.S.R.

\section{ANONYMOUS}

1949. Classification of marine environments. Rep. Comm. Treatise Mar. Ecol. Paleoecol., 9: 2, chart.

BARNARD, K. H.

1927. A monograph of the marine fishes of South Africa. Part 2. Ann. So. Afr. Mus., 21 : 419-1065, 18 pls., 14 text figs.

Bauchot-Boutin, M. L.

1953. Revision synoptique du genre Serrivomer (Anguilliformes). Bull. Mus. Hist. Nat. Paris, 25: 365-367, 4 figs.

1954. Identification de Serrivomer beani Gill et Ryder (Téleostéen Anguilliforme). Bull. Mus. Hist. Nat. Paris, 26:303-306, 2 figs.

\section{Beebe, William}

1934. A half mile down. Nat. Geogr. Mag., 66: 700, 703.

1937. Preliminary list of Bermuda deep-sea fish. Zoologica, 22: 197-208.

BeEBE, W., and Rose, Ruth

1926. "The Arcturus Adventure." Bull. N. Y. Zool. Soc., 29: 43-55, figs.

BERG, LEO S.

1940. Classification of fishes both recent and fossil. Trav. Inst. Zool. Acad. Sci. U.R.S.S., 5: 87-517, 190 figs. 
Bertelsen, E.

1951. The ceratioid fishes. Dana Rep., 39: 1-276, 1 pl., 141 text figs.

BERTIN, LÉON

1934. Les poissons apodes appartenant au sous-ordre des Lyomères. Dana Rep., 3: 1-56, 2 pls., 47 text figs.

1937. Les poissons abyssaux du genre Cyema Günther (Anatomie, Embryologie, Bionomie). Dana Rep., 10: 1-30, 24 figs.

1938. Formes nouvelles et formes larvaires de poissons apodes appartenant au sous-ordre des Lyomères. Dana Rep., 15: 1-26, 2 pls., 17 text figs.

1940. Catalogue des types de poissons du Muséum National d'Histoire Naturelle. Part 2. Dipneustes, Chondrostéens, Holostéens, Isospondyles. Bull. Mus. Hist. Nat. Paris, 12: 244-322.

1944. Synopsis ostéologique et synonymie des poissons de la famille des Serrivoméridés (Apodes, Anguilliformes). Bull. Mus. Hist. Nat. Paris, 16: 101-107, 2 figs.

Bertin, Leon, and Estève, Rolande

1951. Catalogue des types de poissons du Muséum National d'Histoire Naturelle. Part 6. Haplomes, Hétéromes, Catostéomes. Paris. 60 pp.

Bigelow, Henry B., and Schroeder, William C.

1948. Fishes of the western north Atlantic. Part I. Sharks. Mem. Sears Found. Mar. Res., 1, (1), 576 pp., 106 figs.

1953. Fishes of the western north Atlantic. Sawfishes, guitar-fishes, skates, rays and chimaeroids. Mem. Sears Found. Mar. Res., 1, (2), 588 pp., 127 figs.

Bigelow, H. B., and Welsh, William W.

1925. Fishes of the Gulf of Maine. Bull. U. S. Bur. Fish., 40, (1), 567 pp., 278 figs.

Bolin, Rolf, and Myers, George S.

1950. Station records of the Crocker-Stanford Deep-sea Expedition, coast of California, September, 1938. Stanf. Ichth. Bull., 3: 203-208.

Borodin, N. A.

1931. Atlantic deep-sea fishes. Bull. Mus. Comp. Zool., 72: 55-89, 5 pls., 1 text fig.

Bragança, Carlos de

1904. Esqualos obtidos nas costas de Portugal durante as campanhas de 1896 a 1903. Resultados das Investigaçoes scientificas feitas a bordo do yacht Amelia. Ichthyologia, 2, 107 pp., 2 pls. Lisboa.

Brauer, A.

1906. Die Tiefseefische. 1. Systematischer Teil. Wiss. Ergebn. Deutschen Tiefsee Exp. Valdivia, 15, (1), pp. 1-432, 18 pls., 176 text figs. Jena.

BruUn, ANTON Fr.

1953. Problems of life in the deep sea. Geogr. Mag., 26: 247-262, figs.

1955. Life and life conditions in the deepest deep sea. Proc. 8th Pac. Sci. Congr. Philippines, 1953, 1: 399-408, 14 pls.

BURKE, VICTOR

1930. Revision of the fishes of the family Liparidae. Bull. U. S. Nat. Mus., 150: $1-204,110$ figs.

CARUS, J. V.

1893. Prodromus faunae Mediterraneae. 2, $854 \mathrm{pp}$. Stuttgart. 


\section{COLLeTt, RoBert}

1880. Den Norske Nordhavs Expedition 1876-78. Zoologi. Fiske. pp.1-162, 5 pls. Christiania.

1905. Fiske indsamlede under "Michael Sars" Togter i Nordhavet 1900-1902. Rep. Norwegian Fish. Mar. Invest., 2, (3), pp. 1-152, 2 pls.

\section{Cousteau, JaQues-Yves}

1954. To the depths of the sea by bathyscaphe. Nat. Geogr. Mag., 106: $67-79$, figs.

Edgerton, H. E.

1955. Photographing the sea's dark underworld. Nat. Geogr. Mag., 107: 523-537, illus.

Ekman, Sven

1935. Tiergeographie des Meeres. Akad. Verlags. M.B.H. Leipzig, pp. 1-542, 244 figs.

1953. Zoogeography of the sea. pp. 1-417, 121 figs. Sidgwick and Jackson, London.

\section{Essipov, V.}

1937. On the fishes of the polar basin and adjacent depths. Problems of Arctic, Arctic Inst. U.S.S.R., Leningrad, 4: 85-97, 2 figs.

1939. On fishes collected by the ice-breaker Sadko expedition in 1935 . Zool. Zhur., 18, (5), pp. 877-887.

Evermann, Barton Warren, and Goldsborough, Edmund Lee

1907. The fishes of Alaska. Bull. U. S. Bur. Fish., 26: 219-360, pls. 14-42, 144 text figs.

FARRAN, G. P.

1924. Seventh report on the fishes of the Irish Atlantic slope. The macrurid fishes (Coryphaenoididae). Proc. Roy. Irish Acad. Dublin, B36: 91-148, 2 pls., 11 text figs.

Fowler, Henry W.

1936. The marine fishes of West Africa. Bull. Amer. Mus. Nat. Hist., 70, 1493 pp., 567 text figs.

Fraser-BrunNer, A.

1935. New or rare fishes from the Irish Atlantic slope. Proc. Roy. Irish Acad. Dublin, B42: 319-326, figs. 1-5.

1950. Studies in plectognath fishes from the Dana expeditions. I. An interesting new genus of triacanthodid fishes from the Celebes Sea. Dana Rep., 35: $3-8$, figs. $1-5$.

\section{Gilbert, Charles Henry}

1915. Fishes collected by the United States Fisheries Steamer Albatross in southern California in 1904. Proc. U. S. Nat. Mus., 48: 305-380, pls. 14-22.

Gilbert, C. H., and Burke, Charles Victor

1912. Fishes from Bering Sea and Kamchatka. Bull. U. S. Bur. Fish., 30: $33-96,37$ figs.

Gilbert, C. H., and Hubbs, Carl H.

1916. Report on the Japanese macrourid fishes collected by the United States Fisheries Steamer Albatross in 1906, with a synopsis of the genera. Proc. U. S. Nat. Mus., 51: 135-214, pls. 8-11. 
1920. Contributions to the biology of the Philippine Archipelago and adjacent regions. The macrourid fishes of the Philippine Islands and the East Indies. Bull. U. S. Nat. Mus., 100, (1), pp. 369-588, 40 figs.

GILChrist, J. D. F.

1922. List of fishes, etc., procured. Rep. Fish. Mar. Biol. Surv. So. Afr., 2: $7-48$.

Gilchrist, J. D. F., and von Bonde, C.

1924. Deep-sea fishes procured by the S. S. Pickle. Rep. Fish. Mar. Biol. Surv. So. Afr., 3, (7), pp. 1-24, pls. 1-6.

Goode, George Brown, and Bean, Tarleton H.

1895. Oceanic ichthyology. Smithsonian Contr. Knowl., 981, 982, xxxv +553 pp., 417 figs.

\section{GÜNTHER, Albert}

1887. Report on the deep-sea fishes. Report on the scientific results of the voyage of H.M.S. Challenger during the years 1873-76. Zoology, 22, pp. 1-268, 66 pls. Edinburgh.

Hickling, C. F.

1928. The exploratory voyages of the Florence Brierly. Notes on the fish recorded. Ann. Mag. Nat. Hist., (10), 2 : 196-209.

Holt, E. W. L., and Byrne, L. W.

1913. Sixth report on the fishes of the Irish Atlantic slope. The families Stomiatidae, Sternoptychidae and Salmonidae. Rep. Fish. Ireland 1912, Sci. Invest., 1: 1-28, pls. 1, 2, text figs. 1-11.

Hovot, Georges S.

1954. Two and a half miles down. Nat. Geogr. Mag., 106: 80-86, figs.

1955. Le bathyscaphe F.N.R.S. 3 au service de l'exploration des grandes profondeurs. Deep-sea Research, 2: 247-249.

JENSEN, AD. S.

1904. The Lycodinae of north Europe and Greenland. Danish Ingolf Exp., 2, (4), pp. 1-99, 10 pls., map. Copenhagen.

1948. Contributions to the ichthyofauna of Greenland, nos. 8-24. Skr. Univ. Zool. Mus. København, 9: 1-182, 4 pls., 26 text figs.

JOHNSEN, SiguRD

1921. Ichthyologiske Notiser I. Bergens Mus. Aarb., 1918-19, (6), pp. 1-95, 1 pl., 8 text figs.

Jordan, David Starr, and Evermann, Barton Warren

1896-1900. The fishes of north and middle America. Bull. U. S. Nat. Mus., 47, 3136 pp., 958 figs.

Jordan, D. S., EvermanN, B. W. and Clark, H. W.

1930. Check list of the fishes and fishlike vertebrates of north and middle America. Rep. U. S. Comm. Fish., 1928, (2), pp. 1-670.

KoEFoEd, EINAR

1927. Fishes from the sea-bottom. Rep. Sci. Res. M. Sars No. Atl. Deep-sea Exp. 1910, 4, (1), pp. 1-147, 6 pls., 55 text figs. Bergen.

Koumans, F. P.

1953. Biological results of the Snellius Expedition. XVI. The Pisces and Leptocardii. Temminckia, 9: 177-275, 2 figs. 
Krogh, August

1934. Conditions of life at great depths in the ocean. Ecol. Monogr., 4, (4), pp. 430-439.

LÖNNBERG, EINAR

1905. The fishes of the Swedish South Polar Expedition. Wiss. Ergebn. Schwed. Südpol.-Exp. 1901-1903, 5, (6), pp. 1-69, 5 pls., 4 text figs.

LÜTKEN, Chr.

1898. The ichthyological results. Danish Ingolf Exp., 2, (1), pp. 1-38, 4 pls., 2 text figs., 1 map. Copenhagen.

MaCLeish, KenNeth

1954. A vertical voyage to the bottom of the sea. Life, 37, (13), pp. 88-100, figs.

MARShall, N. B.

1951. Bathypelagic fishes as sound scatterers in the ocean. Jour. Mar. Res., 10, (1), pp. 1-17.

1954. Aspects of deep sea biology. 380 pp., 4 pls., text figs. London.

MONOD, THEODORE

1954. Sur un premier essai d'utilisation scientifique du bathyscaphe F.N.R.S.

3. C. R. Acad. Sci. Paris, 238: 1951.

MURRAY, JoHN, and HJORT, JOHAN

1912. The depths of the ocean. $x x+821$ pp., 4 maps, 10 pls., 575 text figs. London.

NoRMAN, J. R.

1930. Oceanic fishes and flatfishes collected in 1925-1927. Discovery Rep., 2: $261-370$, pl. 2, 47 text figs.

1939. Fishes. Sci. Rep. John Murray Exp. 1933-34, 7, (1), 116 pp., 41 figs.

NYBELIN, ORVAR

1948. Fishes collected by the Skagerak Expedition in the eastern Atlantic, 1946. Göteborgs K. Vetensk. Vitt.-Samh. Handl., (B), 5, (16), pp. 1-95, 6 pls., 9 text figs.

1951. Introduction and station list. Rep. Swedish Deep-sea Exp. 1947-1948, Zool., 2, (1), pp. 1-28, 5 figs., map.

1953. Sur la distribution géographique et bathymétrique des brotulidés, trouvés au-dessous de 2000 mètres de profondeur. 14 Intern. Zool. Congr. $3 \mathrm{pp}$. Copenhagen.

Pappenheim, P.

1914. Die Fische der Deutschen Südpolen-Expedition 1901-1903. II. Die Tiefseefische. Deutsche Südpol.-Exp. 1901-1903, 15, Zool., 7: 163-200, pls. 9, 10. Berlin.

PARDI, L.

1951. Sul Bathypterois dubius Vaillant nel Mediterraneo. Monit. Zool. Ital., 58: 125-126, fig.

PARR, Albert Eide

1928. Scientific results of the Third Oceanographic Expedition of the Pawnee, 1927. Deep-sea fishes of the order Iniomi from the waters around the Bahama and Bermuda Islands, with annotated keys to the Sudidae, Myctophidae, Scopelarchidae, Evermannellidae, Omosudidae, Cetomimidae and Rondeletiidae of the world. Bull. Bingham Oceanogr. Coll., 3, (3), pp. 1-193, 43 figs. 
1933. Scientific results of the Third Oceanographic Expedition of the Pawnee, 1927. Deep sea Berycomorphi and Percomorphi from the waters around the Bahama and Bermuda Islands. Bull. Bingham Oceanographic Coll., 3, (6), pp. 1-51, 22 figs.

1937. Concluding report on fishes. With species index for articles 1-7 (Fishes of the Third Oceanographic Expedition of the Pawnee). Bull. Bingham Oceanogr. Coll., 3, (7), pp. 1-79, 22 figs.

1946. The Macrouridae of the western north Atlantic and Central American seas. Bull. Bingham Oceanogr. Coll., 10, (1), pp. 1-99, 28 figs.

1948. The classification of the fishes of the genera Bathylaco and Macromastax, possible intermediates between the Isospondyli and the Iniomi. Copeia, pp. 48-54, 2 figs.

1951. Preliminary revision of the Alepocephalidae, with the introduction of a new family, Searsiidae. Amer. Mus. Nov., 1531: 1-21.

Perluman, David

1952. They found a new world six miles under the sea. Argosy, 335, (6), pp. 19-21, 65-68, figs. Kokomo, Indiana.

REgan, C. T.

1926. The pediculate fishes of the suborder Ceratioidea. Oceanogr. Rep. Danish Dana Exp. 1920-22, 2, 45 pp., 13 pls., 27 text figs.

RICHARD, JULES

1902. Campagne scientifique de la Princesse Alice en 1901. Bull. Soc. Zool. France, 27: 81-104.

1934. Liste générale des stations des campagnes scientifiques du Prince Albert de Monaco, avec neuf cartes des itinéraires et des notes et observations. Rés. Camp. Sci. Monaco, 89: 1-348.

Roule, Louis

1912. Notice sur les Sélaciens conservés dans les collections du Musée Océanographique. Bull. Inst. Océanogr. Monaco, 243, $34 \mathrm{pp}$. (Only reprint seen, 1940, Rés. Camp. Sci. Monaco, 103: 174-200.)

1919. Poissons provenant des croisières du yacht Princesse Alice (1891-1919) et du yacht Hirondelle II (1914). Rés. Camp. Sci. Monaco, 52, 190 pp., 7 pls.

1927. Considerations sur deux espèces abyssales de poissons recemment entrées au Musée Océanographique, et sur la valeur teratologique possible de l'une d'elles. Bull. Inst. Océanogr. Monaco, 497, 12 pp., 2 figs.

1935. Nouvelles observations sur quelques espèces de poissons abyssaux provenant de Madère. Bull. Inst. Océanogr. Monaco, 674, 6 pp.

Roule, Louis, and Angel, Fernand

1933. Poissons provenant des campagnes du Prince Albert 1er de Monaco. Rés. Camp. Sci. Monaco, 86, 115 pp., 4 pls.

Sмiтн, J. L. B.

1949. The sea fishes of southern Africa. 550 pp., 1100 figs. Capetown.

SMITH, SANDERSON

1888. Lists of dredging stations in North American waters from 1867 to 1887. Rep. U. S. Comm. Fish, 1886: 869-1017, 5 charts.

Svetovidov, A. N.

1948. Gadiformes. Fauna S.S.S.R., 9, (4), 221 pp., 72 pls., 39 text figs.

Thomson, C. Wyville, and Murray, John

1895. A summary of the scientific results. Rep. Sci. Res. Voy. Challenger, 2 vols., 1608 pp., 9 pls., 43 charts, 22 diagrams. 


\section{TORTONESE, ENRICO}

1952. Studi sui Plagiostomi. VI. Osservazioni critiche su alcune specie mediterranee. Arch. Zool. Ital., 37: 383-397, 5 figs.

Townsend, Charles $\mathrm{H}$.

1901. Dredging and other records of the United States Fish Commission Steamer Albatross, with bibliography relative to the work of the vessel. Rep. U. S. Comm. Fish, 1900: 387-570, 4 pls.

Townsend, Charles H., and Nichols, John T.

1925. Deep-sea fishes of the Albatross Lower California Expedition. Bull. Amer. Mus. Nat. Hist., 52: 1-20, 4 pls., 4 text figs.

VAILLANT, LÉON

1888. Poissons. Expéditions scientifiques du Travailleur et du Talisman pendant les années $1880,1881,1882,1883.406$ pp., 28 pls. Paris.

WEBER, MAX

1913. Die Fische der Siboga-Expedition. Uitkomsten op Zoologisch, Botanisch, Oceanographisch en Geologisch Gebied verzameld in Nederlandsch OostIndië $1899-1900 \ldots$... Monogr, 56, xii +710 pp., 12 pls., 123 text figs. Leiden.

Zenkevich, L. A., Birschstein, I. R., and Beliaev, G. M.

1954. Study of the fauna of the Kurile-Kamchatka trenches, based on materials of the Pacific Ocean Expedition of the Institute of Oceanography of the Academy of Sciences of the U.S.S.R. Priroda, 43: 61-74, 15 figs.

\section{ZUGMAYER, ERIC}

1911. Poissons provenant des campagnes du yacht Princesse Alice (1901-1910). Rés. Camp. Sci. Monaco, 35, 159 pp., 6 pls.

1933. Appendice: Liste complémentaire des déterminations faites par $M$. Zugmayer, in Roule, L., and ANGEL, F., Poissons provenant des campagnes du Prince Albert 1er de Monaco. Rés. Camp. Sci. Monaco, 86: 79-85. 


\section{ADDENDUM}

After this paper had gone to press a few additional species from Oregon Station 1303 were received, including the three listed below (see p. 93). Data are as follows: Gulf of Mexico, $28^{\circ} 47^{\prime}$ N., $87^{\circ} 50^{\prime}$ W., May 26, 1955, 2104-2194 meters.

Aldrovandia gracilis Goode and Bean. Twenty-one specimens, total length 267-536 mm. (see p. 154).

Aldrovandia pallida Goode and Bean. Twenty specimens, total length 307.5-512 mm. (see p. 154).

Chalinura murrayi Günther. One specimen, total length 730 $\mathrm{mm}$. Not known previously from the western Atlantic (see p. 170). The specimen is a male, larger than any other recorded example, and has a decidedly hump-backed appearance.

It should also be noted that $I$ have received, too late for inclusion, a paper by Nybelin (1954, Union Int. Sci. Biol., (B), 16: 65-71), in which are reported twenty specimens of Bathypterois longipes (see p. 133) caught by the Swedish Deep-sea Expedition (1947-1948) at four localities in the north Atlantic, in depths between 4200 and 5600 meters.

I have found that the depth of capture given for the type of Benthosaurus grallator (p. 131) is perhaps an error. Data for this specimen were given originally as follows: Blake station CLXXIV, in Lat. $24^{\circ} 33^{\prime}$ N., Long. $84^{\circ} 23^{\prime}$ W., at a depth of 1850 fathoms. Sanderson Smith (1888, p. 967) listed Blake station 174 in the Caribbean Sea off Guadeloupe Island, depth 878 fathoms. In the Gulf of Mexico, with the latitude and longitude noted above, Smith (op. cit., p. 963) listed only one station (Agassiz 31), and the depth was said to be 1920 fathoms, If the type of $B$. grallator actually came from a depth of 1920 fathoms its lower limit would then be extended from 3384 to 3512 meters.

Manuscript completed February 8, 1956. 


\section{INDEX}

Principal discussion and tables indicated by bold-faced type.

abyssicola, Raja 100

abyssorum, Barathrites 80, 205

Nematonurus 80,167

acanthias, Dolopichthys 247

Monoceratias 247

Oneirodes 247

acanthirostris, Rhynchoceratias 235

Xenoceratias 235

Acanthochaenus luetkeni 80, 190

Acanthocottus profundorum 222

acanthognathus, Lophodolus $\mathbf{2 5 5}$

Acantholiparis 84

opercularis 228

Acanthonus armatus 92, 202, 219

spinifer 219

acclinidens, Cyclothone 123, 125, 289

Acentrophryne longidens 278

Aceratias edentula 278

indicus 273,274

macrorhinus $272,277,279$

indicus 271,274

mollis 271

acinosa, Caulophryne 233

acrolepis, Hemimacrurus 85, 181

Macrurus 181

Acropomatidae 82, 84, 192

Aegeonichthys appelii 241

aequalis, Coryphaenoides 179, 186, 187

Lionurus 187

Macrurus 187

Nezumia 86,186

aequatoris, Bassogigas 206

Coryphaenoides 177

Macrourus 177

affinis, Aldrovandia 153, 295

Barathronus 221

Chimaera 100

Coryphaenoides 167

Halosauropsis 153

Halosaurus 153

Hydrolagus 100

Macrurus 167

Narcetes 109

Nematonurus 168

Psychichthys 100

africana, Macdonaldia 157

agassizi (agassizii), Alepocephalus 104

Bathysaurus 128

Ipnops 137

Leptochilichthys 110 macrops 110 albatrossus, Macrourus 181

alberti, Hydrolagus 101

albifilosa, Dolopichthys 253

albus, Lycenchelys 80, 197

Lycodes 197

alcocki, Dysalotus 193

Alcockia 84

rostrata 80,207

Aldrovandia affinis 153, 295

anguilliformis 153

goodei 155

gracilis $\mathbf{1 5 4}$

hoskynii 153

macrochir $87,152,155,295$

macrochira 155

pallida 154

phalacra 87,155

rostrata $80,152,153$

sp. 153

alepidotus, Derepodichthys 202

Alepocephalidae 79, 82-84, 102

alepocephalids 85

Alepocephalus agassizi 104

asperifrons 113

atlanticus 105

australis $\mathbf{1 0 5}$

barnardi 105

barnardi 105

edentulus atlanticus 105

fundulus 105

macrolepis 116

macropterus 114

murrayi 115

niger 115

productus 93,104

rostratus $79,86,103,111$

sp. 103,116

(Conocara) macroptera 114

Aleposomus copei 77

Alexterion parfaiti 221

algibarbata, Linophryne $\mathbf{2 7 1}$

allector, Dolopichthys $249,251,254$

Allector cheloniae $\mathbf{2 7 9}$

alta, Macdonaldia 158

altipinnis, Coryphaenoides 178

Macrourus 178

altirostris, Rhynchoceratias 240

altus, Polyacanthonotus $\mathbf{1 5 8}$

alveatus, Bathytroctes $\mathbf{1 0 6}$

alvifrons, Bathytroctes $\mathbf{1 0 7}$

Amacrodon binghami 260 
amissus, Macrias 193

analogus, Dolopichthys 249

andracanthus, Microlophichthys 250

anguilliformis, Aldrovandia 153

Halosaurus 153

anguliceps, Coryphaenoides 177

Macrourus 177

anisacanthus, Dolopichthys 244

Anomalophryne hudsonius 278

antarcticus, Bathydraco $\mathbf{1 9 4}$

Lycenchelys 197

antennatus, Bathypterois $\mathbf{1 3 3}$

Antimora australis 160

microlepis 160

mikrolepis 160

rhina 160

rostrata $85,88,158,159,296$

viola 160

Aphanopus carbo 118

Aphyonus 92

gelatinosus 220

mollis 203

apogon, Borophryne 279, 303

Apogonidae 192

appeli (appelii), Aegeonichthys 241

Himantolophus 241

Apristurus indicus 94

apus, Platytroctes $\mathbf{1 1 6}$

arborifer, Linophryne 273

arborifera, Linophryne 232, 270, 273, 278,303

arcturi, Diabolidium 275

Linophryne $\mathbf{2 7 5}$

argentatus, Gaidropsarus 161

argyresca, Linophryne 277,278

armatus, Acanthonus 92, 202, 219

Macrurus 167

(Nematonurus) 168

Nematonurus 78-80, 88, 167, 297

asper, Coryphaenoides 181

Macrourus 181

Macrurus 167

asperifrons, Alepocephalus 113

Brunichthys 113

asperrimus, Trachonurus 171

Asquamiceps indagatio 112

macrolepis 116

pacificus 112

velaris 112

(Perioceps) pacificus 112

atlanticum, Melanostigma 200

atlanticus, Alepocephalus $\mathbf{1 0 5}$

Dibranchus 231

Lycodes 86, 196, 299

atlantidis, Cryptosparas 265

atratus, Dolopichthys 254

Pentherichthys $\mathbf{2 5 4}$

atricolor antennatus, Bathypterois 133

indicus, Bathypterois 133

phenax, Bathypterois 133

atriconus, Chaenophryne 258

atripectus, Porogadus 214
Atrophacanthus danae 88, 228

atrum, Cyema 88, 151, 294

attenuatus, Halosaurus $\mathbf{1 5 2}$

attritus, Bathytroctes 110, 111

Rouleina 79, 85, 111

Aulastomatomorpha 83

caeruliceps 114

phosphorops 113

Aulastomomorpha caeruliceps 114 phosphorops 113

australis, Alepocephalus $\mathbf{1 0 5}$ barnardi 105

Antimora 160

Synaphobranchus 143, 144

Avocettina 149

elongata 150

infans 91, 149, 293

sp. 150

azurlucens, Himantolophus 241

badia, Raja 100

bairdi, Nezumia 186

bairdii, Gastrostomus 138

Macrurus 186

Saccopharynx 138

Bajacalifornia drakei 108

Barathrites 205

abyssorum 80, 205

iris 204

sp. 203

Barathrodemus manatinus 204 nasutus 204

Barathronus affinis 221

diaphanus 203

parfaiti 221

Barbantus 83 curvifrons 118

barnardi, Alepocephalus 105

Bassogigas 82, 92

aequatoris 206

brucei 80,207

crassus $80,85,205$

digittatus $80,85,206$

gilli 207

grandis $\mathbf{2 0 7}$

koefoedi 206

pterotus 205

(Holcomycteronus) digittatus 207

Bassozetus catena 212

compressus $86,202,209$

elongatus 209

glutinosus 86,210

nasus 209

normalis 93,208

oncerocephalus 210,214

sp. 208

taenia 79, 80, 208

bathybii, Liparis (Paraliparis) 226

Paraliparis 226

bathybius, Histiobranchus 143-145

Paraliparis 226

Synaphobranchus $79,80,143,144,145$ 
bathycoetus, Careproctus $\mathbf{2 2 5}$

Bathydraco 83

antarcticus 194

scotiae 194

Bathydraconidae 83, 84, 194

Bathygadus favosus 164

furvescens 164

longifilis 165

melanobranchus 164

sp. (favosus?) 165

Bathylaco nigricans $80,85,127$

Bathylaconidae 80, 127

Bathymicrops 92

regis $79,80,135,136$

sewelli 80,136

Bathynectes compressus 209

gracilis 212

laticeps 216

Bathyonus catena 212

compressus 209

glutinosus 210

pectoralis 217

taenia 208

(Bassozetus) glutinosus 210

Bathyphasma ovigerum 225

bathyphila, Cyclothone 119

Raja 99

bathyphilum, Gonostoma 88, 119, 280

Neostoma 119

Bathypteroidae 80,130

Bathypterois 130, 135

antennatus 133

atricolor antennatus 133

indicus 133

phenax 133

capensis 134,135

dubius $86,130,131$

filiferus 134, 135

insularum 134, 135

longicauda $77,133,134$

longifilis 132,133

longipes 80,133

pectoralis 132

phenax 93,133

sp. 130

Bathysauropsis 129

gracilis 88, 129

Bathysaurus 128

agassizii 128

ferox 87,128

mollis $79,80,85,87,92,129$

obtusirostris 129

Bathytroctes alveatus $\mathbf{1 0 6}$

alvifrons 107

attritus 110,111

curvifrons 118

drakei 108

grimaldii 108

innesi 117

inspector 107

koefoedi 107 macrolepis 79,106

melanocephalus 110,117

michaelsarsi 107

microlepis 108

mollis 111

nasutus 106

rostratus 105

stomias 109

beani (beanii), Serrivomer 148, 149

beebei, Lasiognathus $\mathbf{2 5 9}$

Bellocia 83

vaillanti 110, 118

Benthosaurus 81, 92, 130 . grallator 93, 131

berglax, Macrourus 163

Bertelsenia gladisfenae 261

bicornis, Chaenophryne 256 Linophryne 275

bifilis, Mancalias 263

bifurcata, Talismania 106

bifurcatus, Nemabathytroctes 106

binghami, Amacrodon 260

Thaumatichthys 260

binocularis, Dolichopteryx 90

bispinosus, Ceratias 242

Ceratias (Diceratias) 242

Diceratias 242

Melanocetus 234

bona-nox, Coryphaenoides 182

Nematonurus 182

Borophryne apogon 279, 303

masculina 277

Bothrocaropsis 83

elongata 199

brasiliensis, Isistius 94

braueri, Xenoceratias 236

Brephostoma 84 carpenteri 192

brevibarbata, Linophryne 273

brevibarbis, Chalinura 80,175 Linophryne 276, 278

Macrurus (Chalinura) 175

brevibarbus, Coryphaenoides 175

brevidorsalis, Synaphobranchus 147

brevifilis, Dolopichthys 245

brevirostris, Rhynchoceratias 240

Xenoceratias 236

Brotulidae 80, 82-84, 202

brotulids $81,82,85$

brucei, Bassogigas 80,207

Holcomycteronus 207

Neobythites 207

Brunichthys 84

asperifrons 113

brunnea, Diplacanthopoma 220

brunneus, Ilyophis 85, 142

bucephalus, Coryphaenoides 176 Macrourus 176

bulbiceps, Maynea 199

bulbosus, Oneirodes 245, 246

Bythites crassus 205 
caeruliceps, Aulastomatomorpha 114 Aulastomomorpha 114

Callionymus 161

camurus, Coryphaenoides 180

Macrourus 180

capensis, Bathypterois 134, 135

Caranactis pumilus 245

carapina, Chalinura $80,173,297$

carapinus, Coryphaenoides 173

Macrurus 173

carbo, Aphanopus 118

Careproctus bathycoetus 225

longifilis 225

opisthotremus 225

ovigerum 225

carlsbergi, Dolopichthys 247

Oneirodes 247

carminatus, Coryphaenoides 176

carminifer, Macrourus 176

carpenteri, Brephostoma 192

carunculatus, Ceratias 264

Cryptopsaras 264

Cataetyx simus 220

catena, Bassozetus 212

Bathyonus 212

Porogadus 212

caudalis, Mixonus 85, 202, 216

Caudania mollis 111

Caulophryne 233

acinosa 233

jordani 232, 233

pelagica 233

polynema 233

pelagica 233

pelagicus 233

polynema 233

ramulosa 233

regani 234

Caulophrynidae 232

Celema nuda 212 subarmata 213

Centrocetus spinulosus 236

Centrophorus coelolepis 97 dumerili 96

squamosus 95,97 dumerili 96

Centrophryne gladisfenae 260 spinulosa 261

Centrophrynidae 232, 261

Centroscymnus coelolepis $86,95,97$, 280

Ceratias bispinosus 242

carunculatus 264

couesii 264

holboelli 261, 265

sessilis 263

xenistius 263

n. sp. 262

shufeldti 262

sp. 265

tentaculatus 263

uranoscopus 262
(Diceratias) bispinosus 242

(Paraceratias) mitsukurii 265

Ceratiidae 231, 232, 261

Ceratioidea 90, 231

ceratioids 91

Ceratocaulophryne regani 234

Cetonurus globiceps 80,189 microps 190

Chaenophryne 244

atriconus 258

bicornis 256

columnifera 258

crenata 256,257

crossata 256

crossota 256, 257

crossotus 257

draco 258,259

draco group 257

fimbriata 259

haplactis 258

intermedia 259

longiceps 256, 257, 258

longiceps group 256

longiceps quadrifilis 257

macractis 258

melanodactylus 258

melanorhabdus 258

pacis 258

parviconus 258

pterolophus 258

quadrifilis 256,257

ramifera 258,259

Chalinura 163

brevibarbis 80,175

carapina 80,173, 297

fernandeziana 171

ferrieri 162,173

filifera 171

guentheri 174, 188, 298

guntheri 174

liocephala 80,171

mediterranea 86,170

murrayi $86,170,171$

europaea 170

serrula 175

simula 80,172

whitsoni 162,173

challengeri, Macdonaldia 156

Notacanthus 156

Chauliodus 83

Chaunacidae 229

Chaunax 229

roseus 229

cheloniae, Allector 279

Chiasmodontidae 193

Chimaera affinis 100

gilberti 101

purpurescens 101

Chimaeridae 100

Chirophryne xenolophus 250

Chlorophthalmidae 129

Chlorophthalmus gracilis 129,130 
cicatrifer, Lycodes 197

cinereus, Coryphaenoides 178

Macrourus 86, 177

cirrifer, Dolopichthys 245

Melanocetus 236, 237, 238

claviger, Dolopichthys 245

coelolepis, Centrophorus 97

Centroscymnus $86,95,97,280$

Coelorhynchus talismani 183 vaillanti 183

columnifera, Chaenophryne 258

compressus, Bassozetus 86, 202, 209

Bathynectes 209

Bathyonus 209

Corynophorus 240

Conchognathus grimaldii 140

Congridae 82, 84, 142

Conocara macroptera 114

murrayi 93,115

werneri 114

conorhynchus, Gymnelis 199

Maynea 199

convergens, Lionurus (Nezumia) 186

Macrurus 186

Nezumia 186

copei, Aleposomus 77

Paraliparis 224, 228

cornutus, Dolopichthys 252

Oneirodes 252

coronata, Linophryne 272, 277

corymbifera, Linophryne $\mathbf{2 7 3}, 278$

Corynolophus globosus 240

reinhardti 240

sagamius 240

Corynophorus compressus 240

Coryphaenoides 163

aequalis $179,186,187$

aequatoris 177

affinis 167

altipinnis 178

anguliceps 177

asper 181

bona-nox 182

brevibarbis 175

bucephalus 176

camurus 180

carapinus 173

carminatus 176

cinereus 178

ferrieri 173

filicauda 184

filifer 171

gigas 168

guentheri 174

hextii 179

hoskynii 180

ingolfi 178

liocephalus 171

longicirrhus 177

mediterraneus 170

murrayi 170

orthogrammus 181 paradoxus 176

rupestris 169

serratus 170,187

serrulus 175

simulus 172

sublaevis 179

variabilis 167

whitsoni 174

wood-masoni 180

(Coelorhynchus) talismani 183

(Macrurus) guntheri 174

Cottidae 82, 222, 223

Cottunculidae 223

Cottunculoides spinosus 223

Cottunculus spinosus 223

couesi (couesii), Ceratias 264

Cryptopsaras 231, 264

Cryptosaras 265

Cryptosparas 265

crassiceps, Hymenocephalus 189

Melamphaes 192

Scopelus 192

crassus, Bassogigas $80,85,205$

Bythites 205

Neobythites 205

crenata, Chaenophryne 256, 257

cristatus, Dolopichthys 244

Oneirodes 244

crossata, Chaenophryne 257

crossota, Chaenophryne 256,257

crossotus, Chaenophryne 257

Cryptolychnus micractis 274

paucidens 278

cryptophthalmus, Leucochlamys 80,222

Cryptopsaras carunculatus 264

couesi 231, 264

mitsukurii 265

normani 265

pennifer 265

valdiviae 265

Cryptosaras couesii 265

Cryptosparas 264, 265

atlantidis 265

Ctenochirichthys longimanus 251

curvifrons, Barbantus 118

Bathytroctes 118

cyclolepis, Dolloa 166

Moseleya 166

Nematonurus 166

Cyclothone acclinidens 123, 125, 289

bathyphila 119

grandis 119

livida 120,281

lusca 122

microdon $90,121,126,282-288$

pygmaea 123,125

obscura 88,127

(Gonostoma) microdon 123

Cyema 151

atrum 88, 151, 294

Cyemidae 151

Cynomacrurus pirei 163 
danae, Atrophacanthus 88, 228

Dolopichthys 252, 253

Himantolophus 240

Rouleina 111

Danaphryne nigrifilis 253

deani, Paraliparis 227

densiramus, Linophryne 274

denticulatus, Nannoceratias 274

denudatum, Gonostoma 119

Derepodichthyidae 83, 84, 202

Derepodichthys 84 alepidotus 202

Dermatias platynogaster 244

Dermatorus melanocephalus 213 trichiurus 213

Diabolidium arcturi 275

diadematus, Dolopichthys 245

diaphanus, Barathronus 203

Dibranchus atlanticus 231

hystrix 230

nasutus 230

nudiventer 230

obscurus 231

Diceratias bispinosus 242 glomerulosus 243

Diceratiidae 232, 242

Dicrolene filamentosa 85, 203 introniger 80 nigra 85,203

Dicromita 215 microphthalma 215 oncerocephala 210

digitatus, Dolopichthys 245

digittatus, Bassogigas 80, 85, 206 Bassogigas (Holcomycteronus) 207 Holcomycteronus 206

dinema, Lophodolus 255

Diogenichthys laternatus 90

diphlegma, Linophryne coronata 272

Diplacanthopoma brunnea 220

(Sarcocara) brunnea 220

dofleini, Simenchelys 140

Dolichopteryx binocularis 90 longipes 90 sp. 90

Dolloa cyclolepis 166

Dolopichthys 251

acanthias 247

albifilosa 253

allector $249,251,254$

analogus 249

anisacanthus 244

atratus 254

brevifilis 245

carlsbergi 247

cirrifer 245

claviger 245

cornutus 252

cristatus 244

danae 252, 253

diadematus 245

digitatus 245 exiguus 249

flagellifer 246

frondosus 245

gladisfenae 260

gracilispinis 250

heteracanthus $244,248,249,251$

heteronema 245

hibernicus 245

implumis 249

inimicus 247

jubatus 253

longicornis 253

luetkeni 248

macronema 247

megaceros 244,253

microlophus 249

mirus 247

mucronatus 253

multifilis 245

niger 252

nigrifilis 253

obtusus 245

pennatus 245

platynogaster 245

plumatus 245

pollicifer 245

ptilotus 245

pullatus 253

schmidti 246

simplex 245

sp. 245, 251, 253

tentaculatus 245

thompsoni 247

thysanophorus 246

venustus 254

(Dermatias) platynogaster 245

(Monoceratias) a canthias 247

dorsalis, Macrourus 167

draco, Chaenophryne 258, 259 group 257

drakei, Bajacalifornia 108

Bathytroctes 108

dubius, Bathypterois $86,130,131$

dumerili, Centrophorus 96

dumerilli, Machephilus 96

Dysalotus alcocki 193

Echinomacrurus 83 mollis 80,190

edentula, Aceratias 278 atlanticus, Alepocephalus 105

Edriolychnus macracanthus 271 radians 271

roulei 271

schmidti $88,232, \mathbf{2 7 0}, 278, \mathbf{3 0 2}$ sp. 271

Einara 83 macrolepis 116

Elasmobranchii 94

Electrona parallela 90

elongata, Avocettina 150

Bothrocaropsis 199 
elongatum, Gonostoma 119

elongatus, Bassozetus 209

Labichthys 150

Enchelybrotula 84 paucidens 218

ensis, Gaidropsarus 86, 162 Motella 162

Onogadus 162

Onos 162

Onus 162

eques, Haloporphyrus 159

Lepidion 159

Eretmichthys ocella 211

pinnatus 210

remifer 211

Ericara niger 115

salmonea 79,115

eschrichti group, Oneirodes 244, 301

eschrichtii, Oneirodes 244,248

Etmopterus princeps 95

pusillus 95

spinax 95

eupogon, Linophryne 274

europaea, Chalinura murrayi 170

Eurypharyngidae 137

Eurypharynx 139

pelecanoides 88, 137, 290

richardi 138

sp. 138,139

(Gastrostomus) pacificus 138

exiguus, Dolopichthys 249

exodon, Gigantactis 268

fabricii, Macrourus 163

facilis, Pellisolus 118

favosus, Bathygadus 164

fernandeziana, Chalinura 171

fernandezianus, Macrurus 171

ferox, Bathysaurus 87, 128

Melanocetus 237, 238

ferrieri, Chalinura 162, 173

Coryphaenoides 173

fierasfer, Lycodapus 201

filamentosa, Dicrolene 85, 203

filibulbosus, Gigantactis $\mathbf{2 6 8}$

filicauda, Coryphaenoides 184 Lionurus $79,80,88,184$

Macrurus 185

filifer, Coryphaenoides 171

filifera, Chalinura 171

filiferus, Bathypterois 134, 135

fimbriata, Chaenophryne 259

fimbriatus, Paraliparis 226

firmisquamis, Nematonurus 163

firthi, Typhloceratias 263

flagellicauda, Grenurus 182

Lycenchelys 198

Lycodonus 198

Macrurus 182

flagellifer, Dolopichthys 246

group, Oneirodes 246

flagellum, Saccopharynx 138 fragilis, Lionurus $\mathbf{1 8 5}$

Macrurus 185

frigida, Ly codes reticulatus 195

frigidus, Lycodes 195, 196, 299

frondosus, Dolopichthys 245

fundulus, Alepocephalus $\mathbf{1 0 5}$

furvescens, Bathygadus 164

fyllae, Raja 98

Gadidae 82, 161

Gadomus longifilis 93, 165

Gadus lepidion 158

Gaidropsarus argentatus 161 ensis 86,162

Gastrostomus 139

bairdii 138

pacificus 138

Gavialiceps microps 149 tinrayei 150

gelatinosum, Melanostigma 200

gelatinosus, Aphyonus 220

Gigantactinidae 232, 266

Gigantactis 266, 267

exodon 268

filibulbosus 268

gracilicauda 267

longicirra 269

macronema 267

microphthalmus 266, 269

ovifer 268

perlatus 268

sexfilis 267

vanhoeffeni 267,268

gigantea, Reganula 263

giganteus, Reganichthys 263

gigas, Coryphaenoides 168

Macrourus 77

Nematonurus 168

gilberti, Chimaera 101

gilli (gillii), Bassogigas 207

Stephanoberyx 190

gladisfenae, Bertelsenia 261

Centrophryne 260

Dolopichthys 260

Spiniphryne 260

globiceps, Centonurus 80, 189

Hymenocephalus 189

Macrurus 189

(Cetonurus) 189

globosus, Corynolophus 240

Himantolophus 240

glomerosus, Oneirodes 243

Paroneirodes 242, 243

glomerulosus, Diceratias 243

Paroneirodes 242

glutinosus, Bassozetus 86, 210

Bathyonus 210

Gonostoma 222

bathyphilum $88, \mathbf{1 1 9}, 280$

denudatum 119

elongatum 119

grande 120 
grandis 120

microdon 121,122

sp. 120

(Cyclothone) microdon 122

Gonostomatidae 88, 119

goodei, Aldrovandia 155

Halosaurus 154

Hymenocephalus 168

Macrurus 167

(Hymenocephalus) 168

(Nematonurus) 168

Nematonurus 168

goodii, Macrurus 167

gracilicauda, Gigantactis 267

gracilis, Aldrovandia 154, 320

Bathynectes 212

Bathysauropsis 88, 129

Chlorophthalmus 129, 130

Moebia 212

Porogadus 212

gracilispinis, Dolopichthys 250

Leptacanthichthys $\mathbf{2 5 0}$

grallator, Benthosaurus 93, 131, 320

grande, Gonostoma 120

grandiceps, Microlepidium 158

Paraliparis 228

grandis, Bassogigas 207

Cyclothone 119

Gonostoma 120

Neobythites 207

Sirembo 207

Grenurus flagellicauda 182

hirundo 182

Grimaldichthys 217

profundissimus $79,80,82,218$

squamosus 80,217

grimaldii (grimaldi), Bathytroctes 108

Conchognathus 140

Grimatroctes 108

Grimatroctes grimaldii 108 microlepis 108

griseus, Hexanchus 94

groenlandicus, Himantolophus 239, 256, 257

guentheri (guntheri), Chalinura 174, 188,298

Coryphaenoides 174

(Macrurus) 174

Lionurus 174

Macruroplus 174

Macrurus 174

Rouleina 111

Sirembo 216

Xenodermichthys 111, 112

Gymnelis conorhynchus 199

Gymnosimenchelys leptosomus 140

\section{Halargyreus 81}

Halieutopsis tumifrons $\mathbf{2 3 0}$

Haloporphyrus eques 159

lepidion 158

eques 159 rostratus 159

viola 159

Halosauridae 80, 152

halosaurids 85

Halosauropsis affinis 153

macrochir 155

phalacrus 156

rostratus 153

Halosaurus affinis 153

anguilliformis 153

attenuatus 152

goodei 154

hoskynii 153

johnsonianus 152

macrochir 155

niger 155

phalacrus 155

rostratus 153

haplactis, Chaenophryne 258

Haplophryne hudsonius 278

mollis $271,273,278$

simus 274

sp. 278

sp. (?Edriolychnus male) 271

triregium 278

Harpadon 128

Harpadontidae 80, 128

Harriotta raleighana 101

hasta, Nettastoma 148

Paraserrivomer 148

Serrivomer 148

Hemimacrurus acrolepis 85, 181

heteracanthus, Dolopichthys 244, 248, 249,251

heteronema, Dolopichthys 245

heterorhynchus, Xenoceratias 236

Hexanchus griseus 94

hexti (hextii), Coryphaenoides 179

Macrourus 179

Tauredophidium 219

hibernicus, Dolopichthys 245

Himantolophidae 232, 239

Himantolophus 239

appeli 241

azurlucens 241

danae 240

globosus 240

groenlandicus 239, 256, 257

kainarae 240

ranoides 240

reinhardtii 239

rostratus 241

hirundo, Grenurus 182

Macruroplus 182

Macrurus 182

Histiobranchus bathybius 143-145

infernalis 77,143

holboelli, Ceratias 261, 265

Holcomycteronus brucei 207 digittatus 206

Holocephali 100

holomelas, Paraliparis 227 
holotrachys, Macrurus 174

Holtbyrnia innesi 117

melanocephalus 117

(Mentodus) polycoeca 118

hoskyni (hoskynii), Aldrovandia 153

Coryphaenoides 180

Halosaurus 153

Macrourus 180

hudsonius, Anomalophryne 278

Haplophryne 278

Hyaloceratias 270, 271, 273, 275, 278 parri 278

Hydrolagus affinis $\mathbf{1 0 0}$

alberti 101

purpurescens 101

Hymenocephalus crassiceps 189 globiceps 189

goodei 168

italicus $80,86,184$

longifilis 165

hyperborea, Raja 98

hystrix, Dibranchus 230

\section{Ilyophidae 142}

Ilyophis brunneus 85,142

implumis, Dolopichthys 249

indagatio, Asquamiceps 112

indicus, Aceratias 273, 274

Aceratias macrorhinus 271, 274

Apristurus 94

Bathypterois atricolor 133

Lophodolus 255

Pentanchus 94

Scylliorhinus 94

(Halaelurus) 94

infans, Avocettina 91, 149, 293

Labichthys 150

Nemichthys 149,150

infernalis, Histiobranchus 77, 143

Synaphobranchus 143

inflaticeps, Macrouroides 92

ingolfi, Coryphaenoides 178

Macrourus 178

inimicus, Dolopichthys 247

Oneirodes 247

innesi, Bathytroctes 117

Holtbyrnia 117

inspector, Bathytroctes 107

insularum, Bathypterois 134, 135

intermedia, Chaenophryne 259

introniger, Dicrolene 80

Ipnoceps pristibrachium 137

Ipnopidae 80, 135

Ipnops 137

agassizi 137

murrayi $80,93,136$

pristibrachium 137

iris, Barathrites 204

Isistius brasiliensis 94

italicus, Hymenocephalus $80,86,184$ Macrurus 184

(Mystaconurus) 184 japonicus, Macrurus 183

jenseni, Raja 98

johnsoni, Melanocetus 234, 235, 301

johnsonianus, Halosaurus $\mathbf{1 5 2}$

jordani, Caulophryne 232, 233

Lycenchelys 197

Lycodes 197

pelagica, Caulophryne 233

polynema, Caulophryne 233

jubatus, Dolopichthys 253

kainarae, Himantolophus 240

Kali macrodon 193

kaupi, Synaphobranchus 93, 145, 291

koefoedi, Bassogigas 206

Bathytroctes 107

Searsia 103

krechi, Melanocetus 234, 236

kroyeri, Mancalias 263

kuhlii, Pontinus 222

labiatus, Macrurus 183

Oxygadus 84, 183

Labichthys elongatus 150 infans 150

Laemonema melanurum 161

laevis, Malacocephalus 179, 184 Xenoceratias 236

Laevoceratias 242 liparis 243

Lasiognathus 260 beebei 259 saccostoma 259

laternatus, Diogenichthys 90

laticeps, Bathynectes 216 Mixonus 80, 214, 216

latifrons, Paraliparis 227

latirhinus, Rhynchoceratias 235 Xenoceratias 235

lecointei, Macrurus 166

Nematonurus 162, 166

lepidion, Gadus 158

Haloporphyrus 158

Lepidion 81, 86, 158

Lota 158

Lepidion eques 159

lepidion 81, 86, 158

rissoi 158

rubescens 158

Lepidoleprus sclerorhynchus 187

Lepidorhinus squamosus 96

Leptacanthichthys gracilispinis 250

Leptocephalus Cyematis atri 151 Gastrostomi Bairdii 138

Histiobranchi infernalis 143

Ilyophidis brunnei 143

sp. 151

sp. F 151

Synaphobranchi pinnati 146

Synaphobranchus infernalis 144

Leptochilichthys agassizi 110 agassizii macrops 110 
macrops 110

Leptoderma macrops 113

leptonema, Rhynchactis 269

leptosomus, Gymnosimenchelys 140

Simenchelys 140

lepturus, Macrourus 167

Nematonurus 167

Leucicorus 84

lusciosus 218

Leucochlamys 83

cryptophthalmus 80,222

sp. 203

leucorhinus, Rhynchoceratias 238,245 , $249,251,254,256,257,261$

Trematorhynchus $245,256,257$

Linophryne 278

algibarbata 271

arborifer 273

arborifera $232,270,273,278,303$

eupogon 274

arcturi 275

argyresca 277,278

bicornis 275

brevibarbata 273

brevibarbis 276,278

coronata 272,277

diphlegma 272

longibarbata 272

corymbifera $\mathbf{2 7 3}, 278$

densiramus 274

eupogon 274

longibarbata 272

lucifer 272

macrodon 276,278

macrorhinus group 276, 277

masculina 273,277

polypogon 276,278

quinqueramosus 273

racemifera 277,278

Linophrynidae 232,270

liocephala, Chalinura 80, 171

liocephalus, Coryphaenoides 171 Macrurus 171

Liocetus murrayi 234

liolepis, Lionurus 85, 185

Macrurus 185

(Lionurus) 185

Lionurus aequalis 187

filicauda $79,80,88,184$

fragilis $\mathbf{1 8 5}$

guntheri 174

liolepis 85,185

parvipes 189

(Nezumia) aequalis 187

convergens 186

parvipes 189

sclerorhynchus 188

lipacantha, Raja fyllae 99

Lipactis megalops 240

tumidus $240,260,274$

Liparidae 81-85, 92, 223

liparids 224
Liparis (Paraliparis) bathybii 226

liparis, Laevoceratias 243

livida, Cyclothone 120, 281

longibarbata, Linophryne 272

longicauda, Bathypterois 77, 133, 134

longicaudatus, Macropharynx 138

longiceps, Chaenophryne 256, 257, 258 group, Chaenophryne 256 Porogadus 214 quadrifilis, Chaenophryne 257

longicirra, Gigantactis $\mathbf{2 6 9}$

longicirrhus, Coryphaenoides 177 Macrourus 177

longicornis, Dolopichthys 253

longidens, Acentrophryne 278

longidentatus, Serrivomer sector 148, 149

longifilis, Bathygadus 165

Bathypterois 132, 133

Careproctus 225

Gadomus 93, 165

Hymenocephalus 165

longimanus, Ctenochirichthys 251

longipes, Bathypterois $80, \mathbf{1 3 3}, 320$ Dolichopteryx 90

longipinnis, Rhynchoceratias 235 Xenoceratias 235

longirostris, Melanocetus 238

longus, Macdonaldia 156

Lophodolus 255

acanthognathus 255

dinema 255

indicus 255

lyra 255

Lota lepidion 158

lucifer, Linophryne 272

luetkeni (lutkeni), Acanthochaenus 80, 190

Dolopichthys 248

Oneirodes 248

lusca, Cyclothone 122

lusciosus, Leucicorus $\mathbf{2 1 8}$

Lycenchelys albus 80,197

antarcticus 197

flagellicauda 198

jordani 197

ophidium 198

platyrhinus 197

Lycodapodidae 83, 84, 201

Lycodapus 83

fierasfer 201

Lycodes albus 197

atlanticus 86, 196, 299

cicatrifer 197

frigidus 195, 196, 299

jordani 197

muraena 198, 199

platyrhinus 196

reticulatus 195

frigida 195

sp. 195,196

terrae-novae 196 
vahlii 195

Lycodonus flagellicauda 198 mirabilis $86,198,299$

ophidium 198

Lyconus pinnatus 163

lyra, Lophodolus 255

macdonaldi, Penopus 215, 216

Porogadus 215

Macdonaldia africana 157

alta 158

challengeri 156

longus 156

rostrata 157

sp. 156

Machephilus dumerilli 96

macracanthus, Edriolychnus 271

macractis, Chaenophryne 258

Macrias 84

amissus 193

macrochir, Aldrovandia $87,152,155$, 295

Halosauropsis 155

Halosaurus 155

macrochira, Aldrovandia 155

macrodon, Kali 193

Linophryne 276, 278

macrolepis, Alepocephalus 116

Asquamiceps 116

Bathytroctes 79,106

Einara 116

macronema, Dolopichthys 247

Gigantactis 267

Macropharynx longicaudatus 138

macrops, Leptochilichthys $\mathbf{1 1 0}$

Leptoderma 113

macroptera, Alepocephalus (Conocara) 114

Conocara 114

macropterus, Alepocephalus 114

macrorhinus, Aceratias 272, 277, 279

group, Linophryne 276, 277

indicus, Aceratias 271, 274

macrostoma, Malacosarcus 80, 191

Scopelus 191

Macrouridae $80,83,84,162$

macrourids $81,82,85$

Macrouroides inflaticeps 92

Macrourus (Macrurus) 163

acrolepis 181

aequalis 187

aequatoris 177

affinis 167

albatrossus 181

altipinnis $\mathbf{1 7 8}$

anguliceps $\mathbf{1 7 7}$

armatus 167

asper 167,181

bairdii 186

berglax 163

bucephalus 176

camurus 180 carapinus 173

carminifer 176

cinereus 86,177

convergens 186

dorsalis 167

fabricii 163

fernandezianus 171

filicauda 185

flagellicauda 182

fragilis 185

gigas 77

globiceps 189

goodei 167

goodii 167

guentheri 174

guntheri 174

hexti 179

hirundo 182

holotrachys 174

hoskyni 180

ingolfi 178

italicus 184

japonicus 183

labiatus 183

lecointei 166

lepturus 167

liocephalus 171

liolepis 185

longicirrhus 177

murrayi 170

orthogrammus 181

paradoxus 176

parvipes 189

rupestris 169

sclerorhynchus $174,175,187,188$

sclerorhynchus var. 174

serratus 187

simulus 172

smiliophorus 187

sublaevis $\mathbf{1 7 9}$

wood-masoni 86,180

(Cetonurus) globiceps 189 sp. 189

(Chalinura) brevibarbis 175

murrayi 170

simulus 172

(Coelorhynchus) talismani 183

(Coryphaenoides) mediterraneus 170 rupestris 169

(Hymenocephalus) goodei 168

(Lionurus) filicauda 185

liolepis 185

(Mystaconurus) italicus 184

(Nematonurus) armatus 168 goodei 168

suborbitalis 166

macrurid larva "A. H." $1 a$ and " $A$. H." 2169

Macruroplus guentheri 174

hirundo 182

sclerorhynchus 188

serratus 187 
Macrurus, see Macrourus

magnus, Nematonurus 163

Malacocephalus laevis 179, 184 sublaevis 179

Malacosarcus 84 macrostoma 80, 191

malispinosus, Monomeropus 203

Malthopsis spinosa 229

manatinus, Barathrodemus 204

Mancalias bifilis 263

kroyeri 263

sessilis 263

shufeldti 262

tentaculatus 262

uranoscopus 262

triflos 263

xenistius 262

Mancalius 262

masculina, Borophryne 277

Linophryne 273, 277

Mastigopterus 84

praetor 217

profundissimus 218

squamosus 217

Maynea bulbiceps 199

conorhynchus 199

mediterranea, Chalinura 86,170

mediterraneus, Coryphaenoides 170

Macrurus (Coryphaenoides) 170

megaceros, Dolopichthys 244,253 Oneirodes 244

megalodontis, Melanocetus 235, 238

Megalopharynx longicaudatus 138

megalops, Lipactis 240

Melamphaes 191

crassiceps 192

nigrescens 88, 191, 298

nycterinus 192

robustus 191

Melamphaidae 191

melanobranchus, Bathygadus 164

melanocauda, Oneirodes 248

melanocephalus, Bathytroctes 110, 117

Dermatorus 213

Holtbyrnia 117

Porogadus 213

Melanocetidae 232, 234

Melanocetus bispinosus 234

cirrifer $236,237,238$

ferox 237238

johnsoni 234, 235, 301

krechi 234,236

longirostris 238

megalodontis 235,238

murrayi $93,232, \mathbf{2 3 4}, 239,300$

niger $234,237,238$

nudus 238

pelagicus 233

polyactis $\mathbf{2 3 8}$

rotundatus 236

sp. 236,265

tumidus 239 vorax 234

(Liocetus) murrayi 234

melanodactylus, Chaenophryne 258

Melanonus 158

melanorhabdus, Chaenophryne 258

Melanostigma atlanticum 200

gelatinosum 200

pammelas 85, 200

melanurum, Laemonema 161

melas, Scymnodon 97

merrimani, Parrichthys 263

metriostomus, Monomitopus 203

michaelsarsi, Bathytroctes 107

micracanthus, Xenoceratias 236

micractis, Cryptolychnus 274

microdon, Cyclothone 90, 121, 126, 282-288

(Gonostoma) 123

Gonostoma 121, 122

(Cyclothone) 122

pygmaea, Cyclothone 123

Microlepidium grandiceps 158

microlepis, Antimora 160

Bathytroctes 108

Grimatroctes 108

Microlophichthys andracanthus 250

microlophus 249, 302

microlophus, Dolopichthys 249

Microlophichthys 249, 302

microphthalma, Dicromita 215

microphthalmus, Gigantactis 266, 269

Monomitopus 215

Penopus(?) 215

Porogadus 214, 215

Sirembo 215,216

Teleotrema 269

microps, Cetonurus 190

Gavialiceps 149

Serrivomer 149

microtrachys, Raja 99

mikrolepis, Antimora 160

miles, Porogadus 93, 211

Miopsaras myops 262

mirabilis, Lycodonus 86, 198, 299

Mirorictus 84

taningi 116

mirus, Dolopichthys 247

mitsukurii, Ceratias (Paraceratias) 265

Cryptopsaras 265

Paraceratias 265

Mixonus caudalis 85, 202, 216

laticeps $80,214,216$

pectoralis 217

Moebia gracilis 212

promelas 214

mollis, Aceratias 271

Aphyonus 203

Bathysaurus 79, 80, 85, 87, 92, 129

Bathytroctes 111

Caudania 111

Echinomacrurus 80, 190 
Haplophryne 271, 273, 278

Talismania 111 monae, Stephanoberyx 86,190

Monoceratias acanthias 247

Monomeropus malispinosus 203

Monomitopus 215

metriostomus 203

microphthalmus 215

oncerocephalus 210

Moridae 158

Moseleya cyclolepis 166

Motella ensis 162

mucronatus, Dolopichthys 253

multifilis, Dolopichthys 245

multiradiatus, Trematorhynchus 251

muraena, Lycodes 198, 199

murrayi, Alepocephalus 115

Chalinura 86, 170, 171, 320

Conocara 93,115

Coryphaenoides 170

Ipnops $80,93,136$

Liocetus 234

Macrurus 170

(Chalinura) 170

Melanocetus 93, 232, 234, 239, 300

Odontomacrurus 163

Myctophidae 88

myops, Miopsaras 262

Nannoceratias denticulatus 274

Narcetes 109

affinis $\mathbf{1 0 9}$

pappenheimi 109

pluriserialis 109

stomias 109

nasus, Bassozetus 209

Typhlonus 80, 219

nasutus, Barathrodemus 204

Bathytroctes 106

Dibranchus 230

Rinoctes 79, 106

Nemabathytroctes bifurcatus 106

Nematonurus abyssorum 80,167

affinis 168

armatus $78-80,88,167,297$

bona-nox 182

cyclolepis 166

firmisquamis 163

gigas 168

goodei 168

lecointei 162, 166

lepturus 167

magnus 163

pectoralis 163

suborbitalis 166

Nematonus pectoralis 217

Nemichthyidae 149

Nemichthys infans 149,150

Neobythites brucei 207

crassus 205

grandis 207 pterotus 205

(Bassogigas) pterotus 206

Neoceratias spinifer 269

Neoceratiidae 232, 269

Neostoma bathyphilum 119 quadrioculatum 122

Nessorhamphus 83

Nettastoma hasta 148

proboscideum 141

procerum 141

Nettastomidae 140

Nettophichthys retropinnatus 146

Nezumia aequalis 86,186

bairdi 186

convergens 186

parvipes 85,189

sclerorhynchus $80,84,86,187,298$

niger, Alepocephalus 115

Dolopichthys 252

Ericara 115

Halosaurus 155

Melanocetus 234, 237, 238

Oneirodes 252

Whitleydea 115

nigra, Dicrolene 85, 203

nigrescens, Melamphaes 88, 191, 298

nigricans, Bathylaco $80,85,127$

nigrifilis, Danaphryne 253

Dolopichthys 253

nilssoni, Skagerakia 131

normalis, Bassozetus 93, 208

normani, Cryptopsaras 265

Notacanthidae 156

Notacanthus challengeri 156

rissoanus 157

nuda, Celema 212

nudiventer, Dibranchus 230

nudus, Melanocetus 238

Porogadus 212, 214

Xenoceratias 238

nycterinus, Melamphaes 192

obesa, Pachycara 80, 201

Pachycarichthys 201

obliquidens, Trematorhynchus 258

obscura, Cyclothone 88, 127

obscurus, Dibranchus 231

obtusirostris, Bathysaurus 129

obtusus, Dolopichthys 245

ocella, Eretmichthys 211

Venefica 142

Odontomacrurus murrayi 163

Ogocephalidae 82,229

oncerocephala, Dicromita 210

oncerocephalus, Bassozetus 210, 214

Monomitopus 210

Sirembo 210

onchorhynchus, Rhynchoceratias 240 , 274

Oneirodes 244

acanthias $\mathbf{2 4 7}$

bulbosus 245,246 
carlsbergi 247

cornutus 252

cristatus 244

eschrichti (eschrichtii) 244, 248

group 244, 301

flagellifer group $\mathbf{2 4 6}$

glomerosus 243

inimicus 247

luetkeni 248

megaceros 244

melanocauda 248

niger 252

schmidti group 246

sp. 255

theodor-tissieri 245

Oneirodidae 232, 243

Onogadus ensis 162

Onos ensis 162

Onus ensis 162 rufus 162

opercularis, Acantholiparis 228

ophidium, Lycenchelys 198

Lycodonus 198

opisthotremus, Careproctus $\mathbf{2 2 5}$

orthogrammus, Coryphaenoides 181 Macrourus 181

ovifer, Gigantactis 268

ovigerum, Bathyphasma 225

Careproctus 225

Oxygadus labiatus 84,183

\section{Pachycara 83}

obesa 80,201

Pachycarichthys obesa 201

pacificus, Asquamiceps 112

Asquamiceps (Perioceps) 112

Eurypharynx (Gastrostomus) 138

Gastrostomus 138

pacis, Chaenophryne 258

pagidostomus, Thaumatichthys $\mathbf{2 6 0}$

pallida, Aldrovandia 154, 320

pammelas, Melanostigma 85, 200

pappenheimi, Narcetes 109

parabeani, Serrivomer 88, 148, 291

Paraceratias mitsukurii 265

paradoxus, Coryphaenoides 176

Macrourus 176

Paraliparis bathybii 226

bathybius 226

copei 224,228

deani 227

fimbriatus 226

grandiceps 228

holomelas 227

latifrons $\mathbf{2 2 7}$

ulochir $\mathbf{2 2 6}$

parallela, Electrona 90

Parapercidae 82, 84, 193

Paraserrivomer hasta 148

parasiticus, Simenchelys 139

Parathunnus 293 parfaiti, Alexterion 221

Barathronus 221

Paroneirodes glomerosus 242, 243

glomerulosus 242

wedli 243

parri, Hyaloceratias 278

Parrichthys merrimani 263

parviconus, Chaenophryne 258

parvipes, Lionurus 189

Macrourus 189

Nezumia 85, 189

parvipinnis, Synaphobranchus pinnatus 146

paucidens, Cryptolychnus 278

Enchelybrotula 218

pectoralis, Bathyonus 217

Bathypterois 132

Mixonus 217

Nematonurus 163

Nematonus 217

pedicellaris, Sciadonus 221

pelagica, Caulophryne 233

Caulophryne jordani 233

pelagicus, Caulophryne 233

Melanocetus 233

pelecanoides, Eurypharynx 88, 137, 290 Saccopharynx 138

Pellisolus 84

facilis 118

pennatus, Dolopichthys 245

pennifer, Cryptopsaras 265

Penopus 216

macdonaldi 215,216

microphthalmus 215

Pentanchus indicus 94

Pentherichthys 254

atratus 254

sp. 254

venustus 254

perlatus, Gigantactis 268

petersoni, Ventrifossa 81

phalacra, Aldrovandia 87, 155

phalacrus, Halosauropsis 156

Halosaurus 155

phenax, Bathypterois 93, 133

phosphorops, Aulastomatomorpha 113 Aulastomomorpha 113

Photocorynus spiniceps $\mathbf{2 7 0}$

Phrynichthys wedli 243

pinnatus, Eretmichthys 210

Lyconus 163

Synaphobranchus 145

pirei, Cynomacrurus 163

platynogaster, Dermatias 244

Dolopichthys 245

platyrhinus, Lycenchelys(?) 197 Lycodes 196

Platytroctes apus 116 procerus 116,117

plumatus, Dolopichthys 245

pluriserialis, Narcetes 109

pollicifer, Dolopichthys 245 
Polyacanthonotus altus $\mathbf{1 5 8}$ rissoanus 157 vaillanti 157

polyactis, Melanocetus $\mathbf{2 3 8}$

polycoeca, Holtbyrnia (Mentodus) 118 Searsia 118

polynema, Caulophryne 233

Caulophryne jordani 233, 234

polypogon, Linophryne 276,278

Pontinus kuhlii 222

Porogadus 215

atripectus $\mathbf{2 1 4}$

catena 212

gracilis $\mathbf{2 1 2}$

longiceps $\mathbf{2 1 4}$

macdonaldi 215,216

melanocephalus 213

microphthalmus 214,215

miles 93, 211

nudus 212,214

promelas 214

rostratus 207

sp. 203

subarmatus 93,213

trichiurus 86,213

praetor, Mastigopterus 217

princeps, Etmopterus 95

Spinax 95

(Etmopterus) 95

pristibrachium, Ipnoceps 137

Ipnops 137

proboscidea, Venefica 87, 141

proboscideum, Nettastoma 141

procera, Venefica 93, 141

procerum, Nettastoma 141

procerus, Platytroctes 116, 117

productus, Alepocephalus 93, 104

profundissimus, Grimaldichthys 79,80 , 82,218

Mastigopterus 218

profundorum, Acanthocottus 222

Xenognathus 115

Zesticelus 222

promelas, Moebia 214

Porogadus 214

Promyllantor 84

purpureus 142

Psychichthys affinis 100

purpurascens 101

Pterodicromita 210

pterolophus, Chaenophryne 258

pterotus, Bassogigas 205

Neobythites 205

ptilotus, Dolopichthys 245

pugnax, Tyrannophryne 250

pullatus, Dolopichthys 253

pumilus, Caranactis 245

purpurascens, Psychichthys 101

purpurescens, Chimaera 101

Hydrolagus 101

purpureus, Promyllantor 142 pusillus, Etmopterus 95

pygmaea, Cyclothone microdon 123, 125

quadrifilis, Chaenophryne 256, 257

quadrioculatum, Nettastonia 122

quinqueramosus, Linophryne 273

racemifera, Linophryne 277,278

radians, Edriolychnus 271

Raja 82

abyssicola 100

badia 100

bathyphila 99

fyllae 98

lipacantha 99

hyperborea 98

jenseni 98

microtrachys 99

Rajidae 82, 98

raleighana, Harriotta 101

ramifera, Chaenophryne 258, 259

ramulosa, Caulophryne 233

ranoides, Himantolophus 240

regani, Caulophryne 234

Ceratocaulophryne 234

Xenoceratias 235

Reganichthys giganteus 263

Reganula gigantea 263

regina, Rhodichthys $86,224,300$

regis, Bathymicrops 79, 80, 135, 136

reinhardti (reinhardtii), Corynolophus 240

Himantolophus 239

remifer, Eretmichthys 211

reticulatus, Lycodes 195

retropinnatus, Nettophichthys 146

rhina, Antimora 160

Rhinochimaera 101

Rhinochimaeridae $\mathbf{1 0 1}$

Rhodichthys 83

regina $86,224,300$

Rhynchactis leptonema $\mathbf{2 6 9}$

Rhynchoceratias acanthirostris 235

altirostris 240

brevirostris 240

latirhinus 235

leucorhinus 238, 245, 249, 251, 254, $256,257,261$

longipinnis 235

oncorhynchus 240,274

rostratus 238,241

richardi, Eurypharynx 138

Rinoctes nasutus 79,106

rissoanus, Notacanthus 157

Polyacanthonotus 157

rissoi, Lepidion 158

robustus, Melamphaes 191

roseus, Chaunax 229

rostrata, Alcockia $80, \mathbf{2 0 7}$

Aldrovandia $80,152,153$

Antimora 85, 88, 158, 159, 296

Macdonaldia 157 
rostratus, Alepocephalus 79, 86, 103, 111

Bathytroctes 105

Haloporphyrus 159

Halosauropsis 153

Halosaurus 153

Himantolophus 241

Porogadus 207

Rhynchoceratias 238, 241

rotundatus, Melanocetus 236

roulei, Edriolychnus 271

Rouleina attritus 79, 85, 111

danae 111

guentheri (guntheri) 111

rubescens, Lepidion 158

rufus, Onus 162

rupestris, Coryphaenoides 169

Macrurus 169

(Coryphaenoides) 169

Saccopharynx bairdii 138

flagellum 138

pelecanoides 138

saccostoma, Lasiognathus 259

sagamius, Corynolophus 240

salmonea, Ericara 79, 115

(Sarcocara) brunnea, Diplacanthopoma 220

schmidti, Dolopichthys 246

Edriolychnus 88, 232, 270, 278, 302

group, Oneirodes 246

Sciadonus 84

pedicellaris 221

sclerorhynchus, Lepidoleprus 187

Lionurus (Nezumia) 188

Macruroplus 188

Macrurus $174,187,188$

Nezumia 80, 84, 86, 187, 298

Scombropidae 192

Scopelus crassiceps 192

macrostoma 191

Scorpaenidae $\mathbf{2 2 2}$

scotiae, Bathydraco 194

Scylliorhinidae 82,94

Scylliorhinus indicus 94

(Halaelurus) indicus 94

Scymnodon melas 97

Searsia koefoedi 103 polycoeca 118

Searsiidae $83,84,102,103,116$

sector, Serrivomer 148, 292

longidentatus, Serrivomer 148, 149

serratus, Coryphaenoides 170, 187

Macruroplus 187

Macrurus 187

Serrivomer 148

beani (beanii) 148, 149

hasta 148

microps 149

parabeani 88, 148, 291

sector 148, 292

longidentatus 148,149
Serrivomeridae $\mathbf{1 4 8}$

serrula, Chalinura 175

serrulus, Coryphaenoides 175

sessilis, Ceratias holboelli 263

Mancalias 263

sewelli, Bathymicrops 80, 136

sexfilis, Gigantactis $\mathbf{2 6 7}$

shufeldti, Ceratias 262

Mancalias 262

Typhlopsaras 262

Simenchelyidae 139

Simenchelys dofleini 140

leptosomus 140

parasiticus 139

simplex, Dolopichthys 245

simula, Chalinura 80,172

simulus, Coryphaenoides 172

Macrurus 172

(Chalinura) 172

simus, Cataetyx 220

Haplophryne 274

Sirembo grandis 207

guentheri 216

microphthalmus 215,216

oncerocephalus 210

Skagerakia nilssoni 131

smiliophorus, Macrurus 187

spinax, Etmopterus 95

Spinax princeps 95

(Etmopterus) princeps 95

spiniceps, Photocorynus $\mathbf{2 7 0}$

spinifer, Acanthonus 219

Neoceratias 269

Spiniphryne gladisfenae $\mathbf{2 6 0}$

spinosa, Malthopsis 229

spinosus, Cottunculoides 223

Cottunculus 223

spinulosa, Centrophryne 261

spinulosus, Centrocetus 236

Squalidae 82, 95

Squalus squamosus 95,96

squamosus, Centrophorus 95

Grimaldichthys 80,217

Lepidorhinus 96

Mastigopterus 217

Squalus 95, 96

var. dumerili, Centrophorus 96

Stephanoberycidae 80, 84, 190

Stephanoberyx 84

gillii 190

monae 86, 190

Stomias 83

stomias, Bathytroctes 109

Narcetes 109

subarmata, Celema 213

subarmatus, Porogadus 93, 213

sublaevis, Coryphaenoides 179

Macrourus 179

Malacocephalus 179

suborbitalis, Macrurus (Nematonurus) 166

Nematonurus $\mathbf{1 6 6}$ 
Synaphobranchidae 80,143

Synaphobranchus australis 143, 144 bathybius $79,80,143,144,145$ brevidorsalis 147 infernalis 143 kaupi 93, 145, 291

pinnatus 145 brevidorsalis 147 parvipinnis 146

(Histiobranchus) bathybius 144

taenia, Bassozetus 79, 80, 208 Bathyonus 208

talismani, Coelorhynchus 183 Coryphaenoides (Coelorhynchus) 183 Macrurus (Coelorhynchus) 183

Talismania bifurcata 106 mollis 111

taningi, Mirorictus 116

Tauredophidium 84 hexti 219

Teleostomi 102

Teleotrema 266 microphthalmus 269 sp. 266

tentaculata, Venefica 142

tentaculatus, Ceratias 263 Dolopichthys 245

Mancalias 262

terrae-novae, Lycodes 196

Thaumanichthys 260

Thaumatichthys binghami 260 pagidostomus $\mathbf{2 6 0}$

theodor-tissieri, Oneirodes 245

thompsoni, Dolopichthys 247

thysanophorus, Dolopichthys 246

tinrayei, Gavialiceps 150

Trachonurus asperrimus 171

Trematorhynchus leucorhinus 245,256 , 257

multiradiatus 251

obliquidens 258

Triacanthodidae 228

trichiurus, Dermatorus 213

Porogadus 86, 213

triflos, Mancalias uranoscopus 263

triregium, Haplophryne 278

tumidus, Lipactis 240, 260, 274

Melanocetus 239

tumifrons, Halieutopsis $\mathbf{2 3 0}$

Typhloceratias firthi 263

Typhlonus 84 nasus 80,219

Typhlopsaras shufeldti 262

Tyrannophryne pugnax 250 ulochir, Paraliparis 226

uranoscopus, Ceratias 262

Mancalias 262

vahlii, Lycodes 195

vaillanti, Bellocia 110, 118

Coelorhynchus 183

Polyacanthonotus $\mathbf{1 5 7}$

valdiviae, Cryptopsaras 265

vanhoeffeni, Gigantactis 267, 268

variabilis, Coryphaenoides 167

velaris, Asquamiceps 112

Venefica 140

ocella 142

proboscidea 87,141

procera 93,141

tentaculata 142

Ventrifossa petersoni 81

venustus, Dolopichthys 254

Pentherichthys 254

viola, Antimora 160

Haloporphyrus 159

vorax, Melanocetus 234

wedli, Paroneirodes 243

Phrynichthys 243

werneri, Conocara 114

Whitleydea niger 115

whitsoni, Chalinura 162, 173

Coryphaenoides 174

wood-masoni, Coryphaenoides 180

Macrourus 86, 180

xenistius, Ceratias holboelli 263

Mancalias 262

Xenoceratias acanthirostris 235

braueri 236

brevirostris 236

heterorhynchus 236

laevis 236

latirhinus 235

longipinnis 235

longirostris 238

micracanthus 236

nudus 238

regani 235

Xenodermichthys guentheri (guntheri) 111,112

Xenognathus profundorum 115

xenolophus, Chirophryne 250

Zesticelus profundorum 222

Zoarcidae $80,82,83,84,194$

zoarcids 85 




$r$ 
Publication 799 



$<$ 

UNIVERSITY OF ILLINOIS-URBANA

$5905 \mathrm{FI}$ C001

FIELDIANA, ZOOLOGYSCHGO

36 1954-58

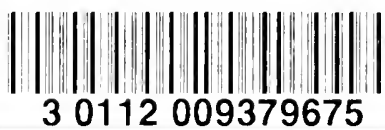

